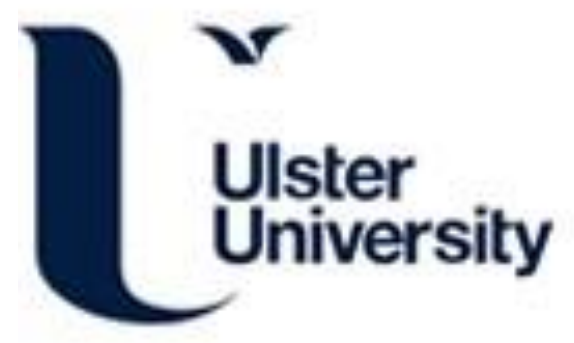

\title{
Physical activity interventions for disease-related physical and mental health during and following treatment in people with non-advanced colorectal cancer
}

McGettigan, M., Cardwell, C., Cantwell, M., \& Tully, M. (2020). Physical activity interventions for disease-related physical and mental health during and following treatment in people with non-advanced colorectal cancer. Cochrane Database of Systematic Reviews, 2020(5), 1-133. [CD012864]. https://doi.org/10.1002/14651858.CD012864.pub2

Link to publication record in Ulster University Research Portal

Published in:

Cochrane Database of Systematic Reviews

Publication Status:

Published (in print/issue): 03/05/2020

DOI:

10.1002/14651858.CD012864.pub2

Document Version

Publisher's PDF, also known as Version of record

\section{General rights}

Copyright for the publications made accessible via Ulster University's Research Portal is retained by the author(s) and / or other copyright owners and it is a condition of accessing these publications that users recognise and abide by the legal requirements associated with these rights.

\section{Take down policy}

The Research Portal is Ulster University's institutional repository that provides access to Ulster's research outputs. Every effort has been made to ensure that content in the Research Portal does not infringe any person's rights, or applicable UK laws. If you discover content in the Research Portal that you believe breaches copyright or violates any law, please contact pure-support@ulster.ac.uk. 


\section{Cochrane}

Library

Cochrane Database of Systematic Reviews

\section{Physical activity interventions for disease-related physical and mental health during and following treatment in people with non- advanced colorectal cancer (Review)}

McGettigan M, Cardwell CR, Cantwell MM, Tully MA

McGettigan M, Cardwell CR, Cantwell MM, Tully MA.

Physical activity interventions for disease-related physical and mental health during and following treatment in people with non-advanced colorectal cancer.

Cochrane Database of Systematic Reviews 2020, Issue 5. Art. No.: CD012864.

DOI: 10.1002/14651858.CD012864.pub2.

www.cochranelibrary.com

Physical activity interventions for disease-related physical and mental health during and following treatment in people with non-advanced colorectal cancer (Review)

Copyright $\odot 2020$ The Cochrane Collaboration. Published by John Wiley \& Sons, Ltd. 
TABLE OF CONTENTS

HEADER 1

ABSTRACT

PLAIN LANGUAGE SUMMARY

SUMMARY OF FINDINGS

BACKGROUND

OBJECTIVES

METHODS

RESULTS

Figure 1.

Figure 2.

Figure 3.

Figure 4.

Figure 5.

Figure 6.

Figure 7.

Figure 8.

Figure 9.

DISCUSSION

AUTHORS' CONCLUSIONS

ACKNOWLEDGEMENTS

REFERENCES

CHARACTERISTICS OF STUDIES

DATA AND ANALYSES

Analysis 1.1. Comparison 1: Physical activity versus usual care for physical function, Outcome 1: Objective measures more than 12 weeks to 6 months follow-up (30-Second Chair Stand Test)

Analysis 1.2. Comparison 1: Physical activity versus usual care for physical function, Outcome 2: Subjective measures more than 12 weeks to 6 months follow-up

Analysis 2.1. Comparison 2: Physical activity versus usual care for disease-related mental health, Outcome 1: Depression: more than 12 weeks to 6 months follow-up

Analysis 2.2. Comparison 2: Physical activity versus usual care for disease-related mental health, Outcome 2: Depression: more than 6 months to 12 months follow-up (HADS)

Analysis 2.3. Comparison 2: Physical activity versus usual care for disease-related mental health, Outcome 3: Anxiety: more than 12 weeks to 6 months follow-up

Analysis 2.4. Comparison 2: Physical activity versus usual care for disease-related mental health, Outcome 4: Anxiety: more than 6 months to 12 months follow-up (HADS)

Analysis 3.1. Comparison 3: Physical activity versus usual care for physical fitness (aerobic fitness), Outcome 1: Up to 12 weeks follow-up

Analysis 3.2. Comparison 3: Physical activity versus usual care for physical fitness (aerobic fitness), Outcome 2: Change from baseline up to 12 weeks follow-up

Analysis 3.3. Comparison 3: Physical activity versus usual care for physical fitness (aerobic fitness), Outcome 3: More than 12 weeks to 6 months follow-up

Analysis 3.4. Comparison 3: Physical activity versus usual care for physical fitness (aerobic fitness), Outcome 4: Change from baseline more than 12 weeks to 6 months follow-up

Analysis 3.5. Comparison 3: Physical activity versus usual care for physical fitness (aerobic fitness), Outcome 5: More than 6 months to 12 months follow-up

Analysis 4.1. Comparison 4: Physical activity versus usual care for physical fitness (hand grip strength), Outcome 1: Up to 12 weeks follow-up (hand dynamometry)

Analysis 4.2. Comparison 4: Physical activity versus usual care for physical fitness (hand grip strength), Outcome 2: More than 12 weeks to 6 months follow-up (hand dynamometry)

Analysis 5.1. Comparison 5: Physical activity versus usual care for physical fitness (flexibility), Outcome 1: More than 12 weeks to 6 months follow-up

Analysis 6.1. Comparison 6: Physical activity versus usual care for cancer-related fatigue, Outcome 1: Up to 12 weeks followup (FACT-F and FACIT-F)

Physical activity interventions for disease-related physical and mental health during and following treatment in people with non- 
Analysis 6.2. Comparison 6: Physical activity versus usual care for cancer-related fatigue, Outcome 2: Change from baseline up to 12 weeks follow-up (FACT-F and FACIT-F)

Analysis 6.3. Comparison 6: Physical activity versus usual care for cancer-related fatigue, Outcome 3: More than 12 weeks to 6 months follow-up

Analysis 6.4. Comparison 6: Physical activity versus usual care for cancer-related fatigue, Outcome 4: More than 6 months to 12 months follow-up

Analysis 7.1. Comparison 7: Physical activity versus usual care for anthropometric measure of weight (kg), Outcome 1: Up to 12 weeks follow-up

Analysis 7.2. Comparison 7: Physical activity versus usual care for anthropometric measure of weight (kg), Outcome 2: Change from baseline up to 12 weeks follow-up

Analysis 7.3. Comparison 7: Physical activity versus usual care for anthropometric measure of weight (kg), Outcome 3: Change from baseline more than 12 weeks to 6 months follow-up

Analysis 7.4. Comparison 7: Physical activity versus usual care for anthropometric measure of weight (kg), Outcome 4: More than 12 weeks to 6 months follow-up

Analysis 8.1. Comparison 8: Physical activity versus usual care for anthropometric measure of waist circumference, Outcome 1: Up to 12 weeks follow-up

Analysis 8.2. Comparison 8: Physical activity versus usual care for anthropometric measure of waist circumference, Outcome 2: More than 12 weeks to 6 months follow-up

Analysis 8.3. Comparison 8: Physical activity versus usual care for anthropometric measure of waist circumference, Outcome 3: Change from baseline more than 12 weeks to 6 months follow-up

Analysis 9.1. Comparison 9: Physical activity versus usual care anthropometric measure of waist to hip ratio, Outcome 1: Up to 12 weeks follow-up

Analysis 9.2. Comparison 9: Physical activity versus usual care anthropometric measure of waist to hip ratio, Outcome 2: Change from baseline up to 12 weeks follow-up

Analysis 9.3. Comparison 9: Physical activity versus usual care anthropometric measure of waist to hip ratio, Outcome 3: More than 12 weeks to 6 months follow-up

Analysis 10.1. Comparison 10: Physical activity versus usual care for anthropometric measure of body fat (\%), Outcome 1: Up to 12 weeks follow-up

Analysis 10.2. Comparison 10: Physical activity versus usual care for anthropometric measure of body fat (\%), Outcome 2 : Change from baseline up to 12 weeks follow-up

Analysis 10.3. Comparison 10: Physical activity versus usual care for anthropometric measure of body fat (\%), Outcome 3: More than 12 weeks to 6 months follow-up

Analysis 10.4. Comparison 10: Physical activity versus usual care for anthropometric measure of body fat (\%), Outcome 4: Change from baseline more than 12 weeks to 6 months follow-up

Analysis 11.1. Comparison 11: Physical activity versus usual care for anthropometric measure of BMI, Outcome 1 : Up to 12 weeks follow-up

Analysis 11.2. Comparison 11: Physical activity versus usual care for anthropometric measure of BMI, Outcome 2: Change from baseline up to 12 weeks follow-up

Analysis 11.3. Comparison 11: Physical activity versus usual care for anthropometric measure of BMI, Outcome 3: More than 12 weeks to 6 months follow-up

Analysis 11.4. Comparison 11: Physical activity versus usual care for anthropometric measure of BMI, Outcome 4: Change from baseline more than 12 weeks to 6 months follow-up

Analysis 12.1. Comparison 12: Physical activity versus usual care for HRQoL, Outcome 1: Up to 12 weeks follow-up ................

Analysis 12.2. Comparison 12: Physical activity versus usual care for HRQoL, Outcome 2: Change from baseline up to 12 weeks follow-up

Analysis 12.3. Comparison 12: Physical activity versus usual care for HRQoL, Outcome 3: More than 12 weeks to 6 months followup

Analysis 12.4. Comparison 12: Physical activity versus usual care for HRQoL, Outcome 4: Change from baseline more than 12 weeks to 6 months follow-up

Analysis 12.5. Comparison 12: Physical activity versus usual care for HRQoL, Outcome 5: More than 6 months to 12 months follow-up

Analysis 13.1. Comparison 13: Physical activity versus usual care for levels of physical activity, Outcome 1: Objective measures up to 12 weeks follow-up (accelerometry moderate to vigorous physical activity mins/per day)

Analysis 13.2. Comparison 13: Physical activity versus usual care for levels of physical activity, Outcome 2: Change from baseline in objective measures up to 12 weeks follow-up (accelerometry moderate to vigorous physical activity) 
Analysis 13.3. Comparison 13: Physical activity versus usual care for levels of physical activity, Outcome 3: Subjective measures up to 12 weeks follow-up

Analysis 13.4. Comparison 13: Physical activity versus usual care for levels of physical activity, Outcome 4: Objective measures more than 12 weeks to 6 months follow-up (accelerometry moderate to vigorous physical activity mins/week)

Analysis 13.5. Comparison 13: Physical activity versus usual care for levels of physical activity, Outcome 5: Subjective measures more than 12 weeks to 6 months follow-up

Analysis 13.6. Comparison 13: Physical activity versus usual care for levels of physical activity, Outcome 6: Subjective measures more than 6 months to 12 months follow-up

ADDITIONAL TABLES

APPENDICES

HISTORY 
[Intervention Review]

\title{
Physical activity interventions for disease-related physical and mental health during and following treatment in people with non-advanced colorectal cancer
}

\author{
Maresa McGettigan ${ }^{1}$, Chris R Cardwell2, Marie M Cantwell2, Mark A Tully 3 \\ ${ }^{1}$ Cancer Prevention, Cancer Focus Northern Ireland, Belfast, UK. ${ }^{2}$ Centre for Public Health, Queen's University Belfast, Belfast, UK. \\ 3 Institute of Mental Health Sciences, School of Health Sciences, Ulster University, Newtownabbey, UK
}

Contact address: Maresa McGettigan, maresa.mcgettigan2@mail.dcu.ie, maresa.mcgettigan@hse.ie.

Editorial group: Cochrane Colorectal Group.

Publication status and date: New, published in Issue 5, 2020.

Citation: McGettigan M, Cardwell CR, Cantwell MM, Tully MA. Physical activity interventions for disease-related physical and mental health during and following treatment in people with non-advanced colorectal cancer. Cochrane Database of Systematic Reviews 2020, Issue 5. Art. No.: CD012864. DOI: 10.1002/14651858.CD012864.pub2.

Copyright @ 2020 The Cochrane Collaboration. Published by John Wiley \& Sons, Ltd.

\section{A B S T R A C T}

\section{Background}

Colorectal cancer is the third most commonly diagnosed cancer worldwide. A diagnosis of colorectal cancer and subsequent treatment can adversely affect an individuals physical and mental health. Benefits of physical activity interventions in alleviating treatment side effects have been demonstrated in other cancer populations. Given that regular physical activity can decrease the risk of colorectal cancer, and cardiovascular fitness is a strong predictor of all-cause and cancer mortality risk, physical activity interventions may have a role to play in the colorectal cancer control continuum. Evidence of the efficacy of physical activity interventions in this population remains unclear.

\section{Objectives}

To assess the effectiveness and safety of physical activity interventions on the disease-related physical and mental health of individuals diagnosed with non-advanced colorectal cancer, staged as T1-4 N0-2 M0, treated surgically or with neoadjuvant or adjuvant therapy (i.e. chemotherapy, radiotherapy or chemoradiotherapy), or both.

\section{Search methods}

We searched the Cochrane Central Register of Controlled Trials (CENTRAL; 2019, Issue 6), along with OVID MEDLINE, six other databases and four trial registries with no language or date restrictions. We screened reference lists of relevant publications and handsearched meeting abstracts and conference proceedings of relevant organisations for additional relevant studies. All searches were completed between 6 June and 14 June 2019.

\section{Selection criteria}

We included randomised control trials (RCTs) and cluster-RCTs comparing physical activity interventions, to usual care or no physical activity intervention in adults with non-advanced colorectal cancer.

\section{Data collection and analysis}

Two review authors independently selected studies, performed the data extraction, assessed the risk of bias and rated the quality of the studies using GRADE criteria. We pooled data for meta-analyses by length of follow-up, reported as mean differences (MDs) or standardised mean differences (SMDs) using random-effects wherever possible, or the fixed-effect model, where appropriate. If a meta-analysis was not possible, we synthesised studies narratively. 


\section{Main results}

We identified 16 RCTs, involving 992 participants; 524 were allocated to a physical activity intervention group and 468 to a usual care control group. The mean age of participants ranged between 51 and 69 years. Ten studies included participants who had finished active treatment, two studies included participants who were receiving active treatment, two studies included both those receiving and finished active treatment. It was unclear whether participants were receiving or finished treatment in two studies. Type, setting and duration of physical activity intervention varied between trials. Three studies opted for supervised interventions, five for home-based self-directed interventions and seven studies opted for a combination of supervised and self-directed programmes. One study did not report the intervention setting. The most common intervention duration was 12 weeks ( 7 studies). Type of physical activity included walking, cycling, resistance exercise, yoga and core stabilisation exercise.

Most of the uncertainty in judging study bias came from a lack of clarity around allocation concealment and blinding of outcome assessors. Blinding of participants and personnel was not possible. The quality of the evidence ranged from very low to moderate overall. We did not pool physical function results at immediate-term follow-up due to considerable variation in results and inconsistency of direction of effect. We are uncertain whether physical activity interventions improve physical function compared with usual care. We found no evidence of effect of physical activity interventions compared to usual care on disease-related mental health (anxiety: SMD - $0.11,95 \%$ confidence interval $(\mathrm{Cl})-0.40$ to 0.18 ; 4 studies, 198 participants; $\mathrm{I}^{2}=0 \%$; and depression: SMD $-0.21,95 \% \mathrm{Cl}-0.50$ to $0.08 ; 4$ studies, 198 participants; $\mathrm{I}^{2}$ $=0 \%$; moderate-quality evidence) at short- or medium-term follow-up. Seven studies reported on adverse events. We did not pool adverse events due to inconsistency in reporting and measurement. We found no evidence of serious adverse events in the intervention or usual care groups. Minor adverse events, such as neck, back and muscle pain were most commonly reported. No studies reported on overall survival or recurrence-free survival and no studies assessed outcomes at long-term follow-up

We found evidence of positive effects of physical activity interventions on the aerobic fitness component of physical fitness (SMD 0.82, $95 \% \mathrm{Cl} 0.34$ to $1.29 ; 7$ studies, $295 ; \mathrm{I}^{2}=68 \%$; low-quality evidence), cancer-related fatigue (MD 2.16, $95 \% \mathrm{Cl} 0.18$ to $4.15 ; 6$ studies, 230 participants; $I^{2}=18 \%$; low-quality evidence) and health-related quality of life (SMD $0.36,95 \% \mathrm{Cl} 0.10$ to $0.62 ; 6$ studies, 230 participants; $\left.\right|^{2}$ $=0 \%$; moderate-quality evidence) at immediate-term follow-up. These positive effects were also observed at short-term follow-up but not medium-term follow-up. Only three studies reported medium-term follow-up for cancer-related fatigue and health-related quality of life.

\section{Authors' conclusions}

The findings of this review should be interpreted with caution due to the low number of studies included and the quality of the evidence. We are uncertain whether physical activity interventions improve physical function. Physical activity interventions may have no effect on disease-related mental health. Physical activity interventions may be beneficial for aerobic fitness, cancer-related fatigue and healthrelated quality of life up to six months follow-up. Where reported, adverse events were generally minor. Adequately powered RCTs of high methodological quality with longer-term follow-up are required to assess the effect of physical activity interventions on the disease-related physical and mental health and on survival of people with non-advanced colorectal cancer. Adverse events should be adequately reported.

\section{PLAIN LANGUAGE SUMMARY}

\section{Physical activity interventions for the physical and mental health of people during and after treatment for bowel cancer}

\section{Background}

Bowel cancer is the third most common cancer diagnosed worldwide. Being diagnosed and receiving treatment for bowel cancer can have a negative impact on a person's physical and mental health. Side effects include reduced fitness levels and increased tiredness. People are also at risk of their cancer returning after treatment and this can cause fear and worry. Research on physical activity programmes in other cancer populations has shown benefits in reducing side effects of treatment. Given that people who are active have a lower chance of developing bowel cancer, physical activity may be beneficial for those with a bowel cancer diagnosis, but the research is not yet clear.

\section{Review question}

This review was undertaken to find out whether physical activity programmes are beneficial for the physical and mental health of people with bowel cancer and whether they are safe.

\section{Key results}

We found 16 studies that included 992 participants, our evidence is current to June 2019. Participants were randomly assigned to receive a physical activity programme or usual care (no physical activity programme). In the included studies, we are unsure whether physical activity programmes improve physical function and we found no effect of physical activity programmes compared to usual care on diseaserelated mental health. No serious adverse events occurred in the eight studies that looked at adverse events. There was inconsistency in reporting and measurement of adverse events. We do not know whether physical activity improves survival at any time point as no studies looked at this. The included studies suggest physical activity programmes may increase aerobic fitness, health-related quality of life (general well-being) and reduce fatigue (tiredness) in the short term. We are unsure of the long-term effects of physical activity interventions on physical function, disease-related mental health, adverse events, physical fitness, fatigue (tiredness), weight, healthrelated quality of life (general well-being) and physical activity levels because no studies assessed this. 


\section{Quality of the evidence}

We rated the quality of the evidence from very low to moderate mainly because of the small number of studies and low number of participants, as well as study limitations.

\section{Conclusion}

The findings of this review should be interpreted with caution due to the low number of studies included and the quality of the evidence. This review shows the need for future high quality research with longer-term follow-up to assess the effects of physical activity interventions on the physical and mental health of people with bowel cancer, especially in relation to safety and survival. 
SUMMARY OF FINDINGS

\section{Summary of findings 1. Physical activity compared with usual care in adults with non-advanced colorectal cance}

Physical activity compared with usual care in adults with non-advanced colorectal cancer

Population: adults with non-advanced colorectal cancer treated surgically or with neoadjuvant or adjuvant therapy, or both

Settings: all but one study undertaken in high-income countries. Included home-based self-directed and supervised physical activity programmes

Intervention: aerobic or resistance training, flexibility or balance training or a combination of these, lasting at least 4 weeks

Comparison: control intervention (usual care or no physical activity intervention)

\begin{tabular}{|c|c|c|c|c|c|c|}
\hline \multirow[t]{3}{*}{ Outcomes } & \multicolumn{2}{|c|}{ Illustrative comparative risks* $(95 \% \mathrm{CI})$} & \multirow{3}{*}{$\begin{array}{l}\text { Relative } \\
\text { effect } \\
(95 \% \mathrm{CI})\end{array}$} & \multirow{3}{*}{$\begin{array}{l}\text { No. of } \\
\text { Partici- } \\
\text { pants } \\
\text { (stud- } \\
\text { ies) }\end{array}$} & \multirow{3}{*}{$\begin{array}{l}\text { Qual- } \\
\text { ity of } \\
\text { the evi- } \\
\text { dence } \\
\text { (GRADE) }\end{array}$} & \multirow[t]{3}{*}{ Comments } \\
\hline & Assumed risk & Corresponding risk & & & & \\
\hline & Usual care & Physical activity & & & & \\
\hline $\begin{array}{l}\text { Physical function } \\
\text { Assessed with: } 30-S e- \\
\text { cond Chair Stand Test } \\
\text { Follow-up: up to } \\
12 \text { weeks (immedi- } \\
\text { ate-term) }\end{array}$ & \multicolumn{2}{|c|}{$\begin{array}{l}\text { We did not pool results due to considerable variability and inconsisten- } \\
\text { cy in direction of effect. Two studies observed no difference between } \\
\text { the physical activity and usual care group for physical function at imme- } \\
\text { diate-term follow-up. Two other studies reported significant improve- } \\
\text { ments in physical function in the physical activity group compared with } \\
\text { usual care }\end{array}$} & & $\begin{array}{l}185 \\
\text { (4 RCTs) }\end{array}$ & $\begin{array}{l}\oplus \oplus \odot \Theta^{a, b} \\
\text { Low }\end{array}$ & $\begin{array}{l}\text { We are uncertain whether physi- } \\
\text { cal activity interventions improve } \\
\text { physical function }\end{array}$ \\
\hline $\begin{array}{l}\text { Disease-related men- } \\
\text { tal health: depression } \\
\text { Assessed with: HADS, } \\
\text { CES-D } \\
\text { Follow-up: more than } \\
12 \text { weeks to } 6 \text { months } \\
\text { (short term) }\end{array}$ & $\begin{array}{l}\text { The mean postintervention } \\
\text { HADS for depression ranged } \\
\text { across control groups from } 2.14 \\
\text { to } 4.72\end{array}$ & $\begin{array}{l}\text { The mean postintervention depres- } \\
\text { sion in the intervention group was } \\
0.84 \text { ( } 2 \text { lower to } 0.32 \text { higher) points } \\
\text { lower than control }\end{array}$ & & $\begin{array}{l}198 \\
\text { (4 RCTs) }\end{array}$ & $\begin{array}{l}\oplus \oplus \oplus \ominus^{b} \\
\text { Moder- } \\
\text { ate }\end{array}$ & $\begin{array}{l}\text { Scores estimated using SMD }-0.21 \\
(-0.50 \text { to } 0.08) \mathrm{g} \\
\text { No evidence of difference in de- } \\
\text { pression in the physical activity } \\
\text { group compared with usual care } \\
\text { group }\end{array}$ \\
\hline $\begin{array}{l}\text { Disease-related men- } \\
\text { tal health: anxiety } \\
\text { Assessed with: HADS, } \\
\text { State-Trait Anxiety In- } \\
\text { ventory }\end{array}$ & $\begin{array}{l}\text { The mean postintervention } \\
\text { HADS for anxiety ranged across } \\
\text { control groups from } 2 \text { to } 3\end{array}$ & $\begin{array}{l}\text { The mean postintervention anxiety } \\
\text { in the intervention groups was } 0.40 \\
\text { points ( } 1.2 \text { lower to } 0.54 \text { higher) low- } \\
\text { er than control }\end{array}$ & & $\begin{array}{l}198 \\
\text { (4 RCTs) }\end{array}$ & $\begin{array}{l}\oplus \oplus \oplus \Theta^{b} \\
\text { Moder- } \\
\text { ate }\end{array}$ & $\begin{array}{l}\text { Scores estimated using SMD }-0.11 \\
(-0.40 \text { to } 0.18) \text { g } \\
\text { No evidence of difference in anxi- } \\
\text { ety in the physical activity group } \\
\text { compared with usual care group }\end{array}$ \\
\hline
\end{tabular}




\begin{tabular}{|c|c|c|c|c|c|c|}
\hline $\begin{array}{l}\text { Follow-up: more than } \\
12 \text { weeks to } 6 \text { months } \\
\text { (short term) }\end{array}$ & & & & & & \\
\hline 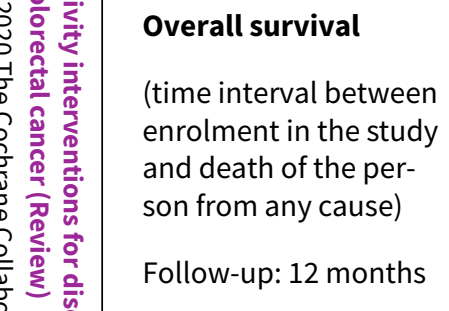 & See comment & See comment & $\begin{array}{l}\text { Not es- } \\
\text { timable }\end{array}$ & & & $\begin{array}{l}\text { The included studies did not re- } \\
\text { port on overall survival }\end{array}$ \\
\hline 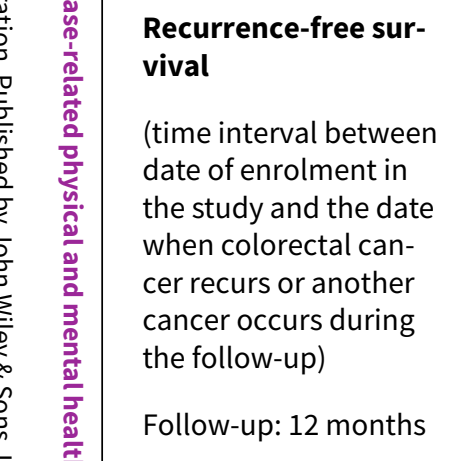 & See comment & See comment & $\begin{array}{l}\text { Not es- } \\
\text { timable }\end{array}$ & & & $\begin{array}{l}\text { The included studies did not re- } \\
\text { port on recurrence-free survival }\end{array}$ \\
\hline $\begin{array}{l}\text { Adverse events } \\
\text { Follow-up: range } 8 \\
\text { weeks to } 11 \text { months }\end{array}$ & \multicolumn{2}{|c|}{$\begin{array}{l}4 \text { studies reported no adverse events, } 3 \text { other studies reported no se- } \\
\text { rious adverse events with } 7 \text { participants experiencing minor adverse } \\
\text { events in one study, } 101 \text { minor adverse events being reported in anoth- } \\
\text { er study and } 39 \text { and } 36 \text { minor adverse events being reported in the inter- } \\
\text { vention and control groups, respectively in another study. } 1 \text { study did } \\
\text { not differentiate between serious and minor adverse events and report- } \\
\text { ed } 9 \text { adverse events in the intervention group and one in the control }\end{array}$} & & $\begin{array}{l}305 \\
\text { (8 RCTs) }\end{array}$ & $\begin{array}{l}\oplus \oplus \Theta \Theta^{c, d} \\
\text { Low }\end{array}$ & \\
\hline 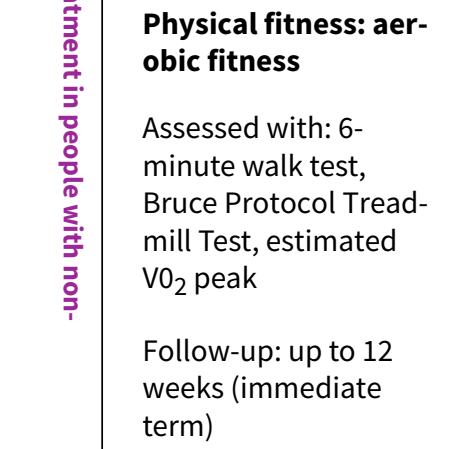 & $\begin{array}{l}\text { The mean postintervention } 6 \text { - } \\
\text { minute walk test score ranged } \\
\text { across control groups from } \\
293.7 \text { to } 588.9\end{array}$ & $\begin{array}{l}\text { The mean postintervention physical } \\
\text { fitness in the intervention group was } \\
59 \text { metres ( } 24.5 \text { to 93.1) higher than } \\
\text { control }\end{array}$ & & $\begin{array}{l}295 \\
\text { (7 RCTs) }\end{array}$ & $\begin{array}{l}\oplus \oplus \ominus \Theta^{\mathrm{a}, \mathrm{e}} \\
\text { Low }\end{array}$ & $\begin{array}{l}\text { Scores estimated using a SMD } \\
0.82(0.34 \text { to } 1.29)^{f} \\
\text { Evidence suggests an improve- } \\
\text { ment in aerobic fitness in the } \\
\text { physical activity group compared } \\
\text { with usual care group }\end{array}$ \\
\hline
\end{tabular}


Cancer-related fatigue

Assessed with: FACIT-F and FACT-F (scale 0-52: higher score indicates lower fatigue)

Follow-up: up to 12 weeks (immediate term)

\section{Health-related quali-} ty of life (HRQOL)

Assessed with: FACT-C FACT-G

(higher score indicates better quality of life)

Follow-up: up to 12 weeks (immediate term)
The mean postintervention cancer-related fatigue score ranged across control groups from 37.1 to 44
The mean postintervention cancer-related fatigue score in the intervention groups was MD 2.16 higher ( 0.18 to 4.15 higher)
Evidence suggests an improvement in cancer-related fatigue in the physical activity group compared with the usual care group

${ }^{\star}$ The basis for the assumed risk (e.g. the median control group risk across studies) is provided in footnotes. The corresponding risk (and its $95 \%$ confidence interval) is based on the assumed risk in the comparison group and the relative effect of the intervention (and its $95 \% \mathrm{Cl}$ ).

CES-D: Centre for Epidemiological Studies Depression Scale; CI: confidence interval; FACIT-F: Functional Assessment of Chronic Illness Therapy-Fatigue; FACT-C: Functional Assessment of Cancer Therapy-Colorectal; FACT-F: Functional Assessment of Cancer Therapy-Fatigue; FACT-G: Functional Assessment of Cancer Therapy-General; HADS Hospital Anxiety and Depression Scale; HRQoL: health-related quality of life; MID : Minmal important difference, MD: mean difference: RCT: randomised controlled trial; SD: standard deviation; SMD: standardised mean difference (used when studies assess the same outcome but measure it in a variety of ways).

GRADE Working Group grades of evidence

High quality: further research is very unlikely to change our confidence in the estimate of effect.

Moderate quality: further research is likely to have an important impact on our confidence in the estimate of effect and may change the estimate.

Low quality: further research is very likely to have an important impact on our confidence in the estimate of effect and is likely to change the estimate.

Very low quality: we are very uncertain about the estimate.

aDowngraded one level due to indirectness (applicability of results to those undergoing active treatment).

bDowngraded one level due to imprecision (small sample size).

'Downgraded one levels due to inconsistency in reporting and measuring and numbers of adverse events reported.

dDowngraded one level due to indirectness (reporting adverse events and not reporting whether these are 'related' or 'unrelated' to the intervention).

eDowngraded one level due to risk of bias (lack of allocation concealment and blinding of outcome assessor).

fAnalysed with SMD and back estimated to MD to enable interpretation. SD for performing the calculation was obtained from study by Lee 2017.

gAnalysed with SMD and back estimated to MD to enable interpretation. SD for performing the calculation was obtained from study by Van Vulpen 2016 


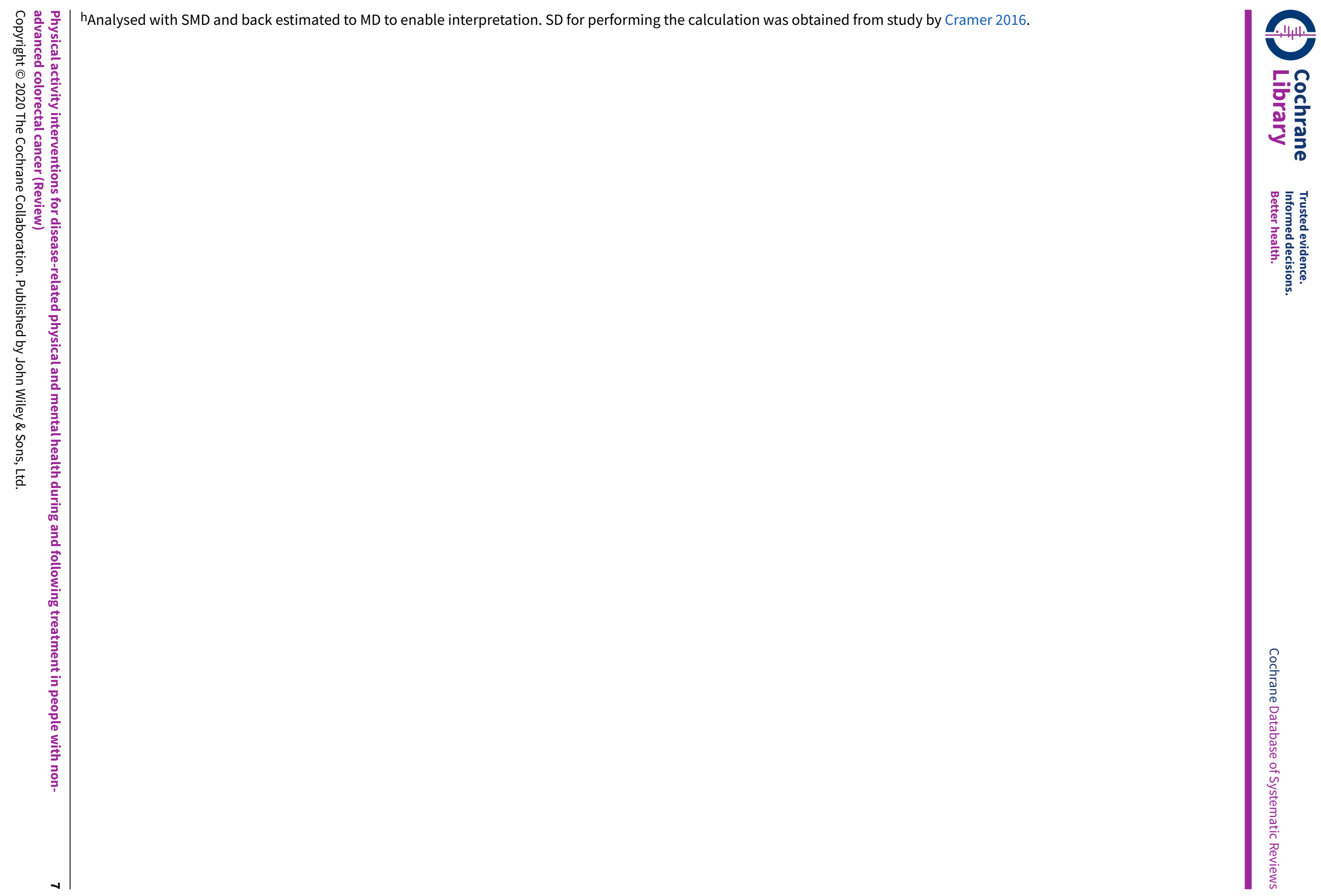




\section{B A C K G R O U N D}

\section{Description of the condition}

Colorectal cancer is the third most commonly diagnosed cancer and the second leading cause of cancer death worldwide, accounting for an estimated 881,000 deaths in 2018 (GLOBOCAN 2018). Incidence and mortality rates vary globally, with higher incidence and lower mortality rates in higher-income countries (Arnold 2017; GLOBOCAN 2018; Stewart 2014). In general, incidence is higher in men than women and is strongly linked with age, with highest incidence among people aged 65 to 74 years (Howlader 2016). Incidence is currently stabilising in high-income countries, however a two-fold cumulative increase in incidence is expected by 2025 , due to increasing incidence in low- to middle-income countries. With development, comes the adoption of more inactive lifestyles and unhealthy dietary habits; established risk factors for colorectal cancer (Stewart 2014). This is expected to increase the global burden of colorectal cancer, which may be compounded by a lack of health service resources in low- and middle-income countries to deal with the escalation in incidence (Stewart 2014).

Five-year survival from colon and rectal cancer has reached $60 \%$ or more in 22 countries worldwide (Allemani 2015). Between 1989 and 2011, colorectal cancer mortality rates decreased by more than $25 \%$ and $30 \%$ in men and women, respectively in highincome countries in Northern and Western Europe, but increased in most Eastern European countries (Ouakrim 2015). Similar trends are evident globally, with decreasing mortality rates in highincome countries, including Australia, Canada (Coleman 2011), the USA (Ryerson 2016), and Japan (Arnold 2017), and contrasting increasing mortality rates in low- and middle-income regions, such as Latin America and the Phillipines (Arnold 2017). These disparities are not easily explained and are likely due to differences in access to diagnostic and treatment services (Haggar 2009), with advancements in treatment and early detection contributing to decreasing mortality in high-income countries (Coleman 2011; Stewart 2014).

Although treatments are advancing, anti-cancer therapies are associated with a range of adverse physiological and psychological side effects, which affect morbidity and mortality (Devin 2016a). Surgical resection is the primary treatment modality for stage I-III (T1-4 N0-2 M0) colorectal cancer, with systemic chemotherapy or radiotherapy (more often in rectal cancer), or both, given either in the adjuvant or neoadjuvant setting in stage III and high risk stage II patients (El-Shami 2015; Labianca 2010). Major abdominal surgery alone has been associated with declines in physical function (Schroeder 1991), and fatigue (Christensen 1982). Cancer-related fatigue affects between $60 \%$ to $96 \%$ of people with cancer during and following chemotherapy, radiotherapy or surgery (Cramp 2012; Thomas 2014; Wagner 2004). It is a distressing symptom defined as a sense of "physical tiredness or exhaustion related to cancer or cancer treatment" (NCCN 2016), which can interfere with one's ability to carry out daily activities (Curt 2000), and negatively affect mood and quality of life (Stone 2008). Cancer-related fatigue is present in some colorectal cancer survivors at four years following diagnosis (Schneider 2007). Physical inactivity has been identified as both a risk factor for (Bower 2014), and a consequence of (Lynch 2010) cancer-related fatigue.

Declines in cardiorespiratory fitness can occur following treatment for colorectal cancer (Devin 2016a; West 2014a). Lower levels of cardiorespiratory fitness are linked with higher rates of cancerspecific morbidity and mortality (Peel 2009; Schmid 2015), and can predict morbidity after colonic and rectal surgery (West 2014b; West 2014c). Furthermore, people with colorectal cancer may be susceptible to sarcopenic obesity (obesity with depleted muscle mass), which is associated with poorer functional status and poorer survival rates (Prado 2008; Wang 2017). These adverse effects, alone or in combination can impact adversely on a patient's quality of life and subsequent physical activity levels (Cramer 2014a). Colorectal cancer survivors are also at an increased risk of developing second colorectal cancers (Green 2002; Markle 2010), non-colorectal cancers (Birgisson 2005), and other comorbidities (Denlinger 2011).

Concerns surrounding recurrence are common, affecting over half of cancer patients at one year following diagnosis (Baker 2005). Even at five years following surgery for colorectal cancer, survivors have concerns surrounding recurrence (Custers 2016). A significant minority of colorectal cancer patients and longer-term survivors of colorectal cancer ( 2 or more years postdiagnosis) experience clinically meaningful levels of psychological distress, including symptoms of anxiety and depression or reduced mental wellbeing (Mosher 2016). Colorectal cancer survivors report high quality of life at five years or longer postdiagnosis, but have higher rates of depression than age-matched populations (Ramsey 2002). Psychological outcomes vary greatly in this population, poorer psychological outcomes have been linked with the presence of existing comorbidities (Lynch 2008; Ramsey 2002), worse general health (Yost 2008), and lower socioeconomic status (Ramsey 2002). Levels of anxiety and depression are reported to be higher in people who undergo surgery with adjuvant chemotherapy or radiotherapy compared with surgery alone (Pereira 2012).

\section{Description of the intervention}

Physical activity interventions were the focus of this review. Physical activity is defined as any bodily movement produced by contraction of skeletal muscle that results in energy expenditure above resting energy expenditure (ACSM 2009; Caspersen 1985). For the purpose of this review the term 'physical activity interventions' included 'exercise interventions'. Exercise is a subset of physical activity that is planned, structured and repetitive, done to improve or maintain one, or more of the components of physical fitness (ACSM 2009; Caspersen 1985). Physical activity interventions may be less structured than exercise interventions and often focus on promoting the integration of activities into daily life (e.g. gardening, walking or active travel). Physical activity interventions may be self-directed or supervised by a healthcare professional. They can involve aerobic or resistance training, flexibility or balance training, or a combination of these, can take place in any setting and can be individual or group based, or both. No restrictions were made regarding frequency, intensity, time or type of physical activity intervention included. Interventions were included if they lasted a minimum of four weeks, this was to exclude studies on the acute effects of physical activity.

Physical activity interventions are not currently delivered as part of standard practice during or following treatment for colorectal cancer. Early postoperative mobilisation is, however, strongly recommended, as part of the Enhanced Recovery After Surgery (ERAS) guidelines following colorectal surgery, encouraging patients to be out of bed for two hours on the day of surgery and six hours per day, thereafter until discharge (Lassen 
2009). The American College of Sports Medicine (Schmitz 2010), the American Cancer Society (Rock 2012), and the British Association of Sport and Exercise Science (BASES 2011) guidelines confirm that exercise can be safely performed during and following cancer treatment in the general cancer population. Specific guidance statements on physical activity interventions during and following treatment for colorectal cancer have not yet been published, due to lack of evidence on adverse effects and lack of safety data (Schmitz 2010). Side effects of treatments (cancer-related fatigue, peripheral neuropathy, immune suppression, digestion issues, bowel dysfunction (including faecal incontinence) and urinary incontinence) may increase the risk of adverse events during physical activity. These side effects may represent barriers to physical activity participation (Denlinger 2009; Denlinger 2011; Rock 2012; Schmitz 2010). Indeed, chronic diarrhoea is a side effect that has been associated with limitations in activity and negative body image (Schneider 2007). The presence of a stoma is also associated with diminished body image (Hong 2014). These side effects have been highlighted as factors to consider when prescribing physical activity. Existing comorbidities (most commonly cardiovascular disease, musculoskeletal problems and lung or breathing problems), particularly in older people with colorectal cancer have been highlighted as other factors requiring consideration, to reduce the risk of injury and adverse events (Denlinger 2009; Rock 2012; Schmitz 2010).

\section{How the intervention might work}

Physical activity has been proposed as non-pharmacologic intervention to attenuate the negative physiologic and psychologic effects of treatment in people with cancer (Courneya 2007; Schmitz 2005). There is a growing body of evidence from Cochrane and nonCochrane systematic reviews demonstrating the positive impact of physical activity both during and following cancer treatment (Galvao 2005; Knols 2005; Schmitz 2005; Speck 2010). Exercise training improves cardiorespiratory fitness and muscle strength (Schmitz 2005; Speck 2010), overall health-related quality of life (HRQoL) (Knols 2005; Mishra 2012a; Mishra 2012b), and cancerrelated fatigue in the general cancer population during and following cancer treatment (Cramp 2012; Furmaniak 2016; Speck 2010), and physical functioning during treatment (Mishra 2012a). Through improved cardiorespiratory fitness and muscle strength, physical activity may help address the physical deconditioning associated with cancer treatments (Schmitz 2005; Speck 2010), and help manage cancer-related fatigue (Al-Majid 2009; Cramp 2012). Physical activity may also help the emotional and mental aspects of cancer-related fatigue (Al-Majid 2009; Cramp 2012). Benefits of exercise interventions on psychological well-being (Knols 2005), anxiety and depression show positive trends but the evidence is not consistent (Cramp 2012; Furmaniak 2016; Mishra 2012a).

Cardiorespiratory fitness has been highlighted as an independent predictor of cancer mortality risk. Higher cardiorespiratory fitness is associated with a significant reduction in total cancer mortality (Schmid 2015), and colorectal cancer mortality (Peel 2009). Peel and colleagues report that men with at least a moderate fitness level had a $42 \%$ lower risk of colorectal mortality compared with men with a low cardiorespiratory fitness level. Evidence from observational studies suggest that physical activity is associated with overall and disease-free survival (Haydon 2006; Meyerhardt 2006; Meyerhardt 2009), in both colon and rectal cancer patients.
There is consistent evidence linking physical activity to reduced colon cancer risk (Leitzmann 2015; Wolin 2009). A meta-analysis of 52 studies found an inverse association between physical activity and colon cancer, with an overall relative risk reduction of $24 \%$ (Wolin 2009). This is consistent with findings of an earlier metaanalysis of 19 cohort studies, which demonstrated a lower risk of colon cancer of $22 \%$ and $29 \%$ in physically active men and women, respectively (Samad 2005). Conversely, there appears to be no consistent association between physical activity and rectal cancer risk (Robsahm 2013).

The exact biological mechanisms for the observed benefit of physical activity for the prevention and secondary prevention of colorectal cancer are not fully understood. Various mechanisms have been proposed. Physical activity may reduce carcinogen exposure in the mucosa through decreased gastrointestinal transit time (Quadrilatero 2003; Slattery 2003), may alter prostaglandin levels (prostaglandins are unsaturated, free fatty acids that affect colonic function) (Quadrilatero 2003), and may alter the insulinlike growth factor (IGF) pathway (Denlinger 2011; Fairey 2003). In people with colorectal cancer, moderate-intensity exercise has resulted in reduced levels of urinary markers of oxidative damage (Allgayer 2008), and decreased interleukin-1 receptor agonist (Allgayer 2004a), which may enhance immune function. Oxidative DNA damage is thought to be involved in tumour formation and may be associated with malignant transition and recurrence (Allgayer 2008). IGF-1 is important for cellular proliferation and survival (Hursting 2010), higher levels of which may be associated with increased risk of colorectal cancer (Giovannucci 2000), but this association remains elusive. Decreases in IGF and increases in IGFbinding proteins have been observed following exercise training in breast cancer survivors, which may be clinically relevant for the colorectal cancer population (Fairey 2003)

Physical activity may therefore be potentially effective in improving overall and recurrence-free survival. Indeed, given that regular physical activity can decrease the risk of colon cancer and has improved cardiorespiratory fitness, muscle strength, HRQoL and cancer-related fatigue in other cancer populations, it may be of clinical relevance for the colorectal cancer control continuum.

\section{Why it is important to do this review}

Colorectal cancer is a major public health problem. With the projected increasing incidence of colorectal cancer in lowand middle-income regions, increasing mortality rates in lowand middle-income countries and 3.5 million colorectal cancer survivors worldwide (Stewart 2014), there is a need to develop effective interventions that aid physical and psychological recovery, help alleviate treatment side effects and increase overall and recurrence-free survival. The Lancet Oncology commission has prioritised the reduction in morbidity and mortality associated with cancer, with a focus on "less toxic", "cost-effective" interventions (Sullivan 2011). There is, therefore, a need for a greater understanding of the effects of physical activity interventions on the disease-related physical and mental health of individuals with colorectal cancer, for policy, practice and for consumers.

To date, there are currently two published, non-Cochrane systematic reviews on exercise interventions for people with colorectal cancer (Cramer 2014b; van Rooijen 2018). In the review by Cramer 2014b no recommendations regarding exercise as a routine intervention for people with colorectal cancer were

Physical activity interventions for disease-related physical and mental health during and following treatment in people with non- 
made due to insufficient evidence and lack of safety data. The review undertaken by Cramer and colleagues was limited to individuals who had completed treatment. The second review by van Rooijen and colleagues was undertaken in participants undergoing treatment, and highlighted the limited evidence of exercise training during treatment for colorectal cancer. Six out of seven studies included mixed-cancer populations, three of these studies were not RCTs. This review is restricted to RCTs only and includes individuals who are receiving adjuvant therapy in addition to those who have finished treatment; no previous review has included such a population. This review will update current evidence and include emerging evidence in relation to physical activity interventions for individuals with colorectal cancer and so identify current evidence gaps.

\section{O B J E C T IVES}

To assess the effectiveness and safety of physical activity interventions on the disease-related physical and mental health of individuals diagnosed with non-advanced colorectal cancer, staged as T1-4 N0-2 M0, treated surgically or with neoadjuvant or adjuvant therapy (i.e. chemotherapy, radiotherapy or chemoradiotherapy), or both.

\section{METHODS}

\section{Criteria for considering studies for this review}

\section{Types of studies}

We considered randomised control trials (RCTs) and cluster-RCTs comparing physical activity interventions to usual care or no physical activity intervention for inclusion in this review.

\section{Types of participants}

We included studies that evaluated the effect of physical activity interventions, on adults (aged 18 years or over), regardless of gender, diagnosed with non-advanced colorectal cancer, staged as T1-4, N0-2, M0, treated surgically or with neoadjuvant or adjuvant therapy (i.e. chemotherapy, radiotherapy, chemoradiotherapy), or both. We included studies that examined physical activity interventions delivered during adjuvant therapy, following adjuvant therapy or following surgery alone. We excluded studies that included participants with other cancer types (unless outcomes for colorectal cancer were reported separately), and studies that included participants who were more than five years postdiagnosis.

\section{Types of interventions}

We compared physical activity interventions separately to no physical activity intervention or to usual care. Participants in both the control and intervention arms received the same usual care. Physical activity sessions could take place in any setting, be supervised, self-directed or both, could be individual or group based, or a combination of both. Physical activity modalities could include aerobic or resistance training, flexibility and balance training or a combination of these. No restrictions were made regarding frequency, intensity, time or type of exercise or physical activity intervention. We only included studies with interventions that lasted a minimum of four weeks in duration, to exclude studies on the acute effects of physical activity. We excluded studies with a prehabilitation component. We included studies that provided health education materials or seminars only if the physical activity intervention was the main intervention in the study. We recorded specific details on the intervention according to the FITT-VP (frequency intensity, time, type, volume, progression) principle (ACSM 2014). We classified physical activity intensity as mild, moderate or vigorous based on the rate of perceived exertion, heart rate or metabolic equivalents report (ACSM 2014), and used the author's classification of mild, moderate, or vigorous when a quantitative measure was unavailable.

\section{Types of outcome measures}

We extracted information for the primary and secondary outcomes at all available time points. We sought to analyse overall survival and recurrence-free survival at 12 months, three years and five years. We analysed the other primary and secondary outcomes according to the length of follow-up: up to 12 weeks after baseline (immediate); more than 12 weeks but less than or equal to six months after baseline (short term); more than six months but less or equal to 12 months after baseline (medium term) and more than 12 months after baseline (long term).

\section{Primary outcomes}

1. Physical function (e.g. Karnofsky Performance Status Scale; Eastern Cooperative Oncology Group Scale; timed chair rise test; timed 'Up \& Go' test) or other valid instruments

2. Disease-related mental health (e.g. Hospital Anxiety and Depression Scale (HADS); Beck Depression Index (BDI))

3. Adverse events (participants experiencing at least 1 adverse event, e.g. injury, death, adverse events resulting in discontinuation of the intervention)

\section{Secondary outcomes}

1. Overall survival (time interval between enrolment in the study and death of the person from any cause)

2. Recurrence-free survival (time interval between date of enrolment in the study and the date when colorectal cancer recurs or another cancer occurs during the follow-up)

3. Physical fitness (e.g. cardiorespiratory endurance (6-minute walk test; 10 -metre shuttle walk test; $\mathrm{V}_{2}$ peak or muscle strength (dynamometry; 1 repetition maximum; 5 repetition maximum) or another valid instrument

4. Cancer-related fatigue (e.g. Functional Assessment of Chronic Illness Therapy-Fatigue (FACIT-F); Schwartz Cancer Fatigue Scale (SCFS); Brief Fatigue Inventory (BFI); Piper Fatigue Scale (PFS))

5. Anthropometric measurements (e.g. weight, body mass index (BMI), body composition, waist measurement, skin-fold measurement)

6. HRQoL (e.g. European Organization for Research and Treatment of Cancer Quality of Life Questionnaire Core 30 (EORTC QLQC30); Medical Outcomes Study Short Form-36 General Health Survey (SF-36); Functional Assessment of Cancer TherapyColorectal scale (FACT-C))

7. Levels of physical activity (e.g. physical activity questionnaires (International Physical Activity Questionnaire (IPAQ), Global Physical Activity Questionnaire (GPAQ) or objective measures of physical activity using pedometers or accelerometers)

Physical activity interventions for disease-related physical and mental health during and following treatment in people with non- 


\section{Search methods for identification of studies}

\section{Electronic searches}

We searched the following electronic databases between 6 June 2019 and 14 June 2019 up to the latest issue, with no language or date restrictions to identify relevant RCTs and cluster-RCTs for this review.

1. The Cochrane Central Register of Controlled Trials (CENTRAL, in the Cochrane Library) (Appendix 1) (inception to present)

2. Ovid MEDLINE(R) Epub Ahead of Print, In-Process \& Other NonIndexed Citations, Ovid MEDLINE(R) Daily and Ovid MEDLINE(R) (1946 to Present) (Appendix 2)

3. Ovid Embase (1974 to present) (Appendix 3)

4. CINAHL (in EBSCOhost 1982 to present)

5. Web of Science (1970 to present)

6. PsycINFO (1806 to present)

7. Open Grey (formerly SIGLE) (1980 to present)

8. PEDro (1999 to present)

The searches were conducted by Cochrane Colorectal Cancer's Information Specialist and a review author (MAT).

\section{Searching other resources}

We searched clinical trials registries separately on 6 June 2019 for ongoing studies and study protocols of:

1. Clinical.trials.gov (www.clinicaltrials.gov);

2. the World Health Organization International Clinical Trials Registry Platform (WHO ICTRP) (apps.who.int/trialsearch/);

3. the EU Clinical Trials Register (www.clinicaltrialsregister.eu/); and

4. CenterWatch (www.centerwatch.com).

We screened reference lists of all included studies and any relevant systematic reviews identified. We handsearched conference and meeting abstracts of relevant organisations including:

1. American Society of Clinical Oncology (ASCO);

2. European Society for Medical Oncology (ESMO);

3. American College of Sports Medicine (ACSM);

4. BIT's Annual World Cancer Congress;

5. European Multidisciplinary Colorectal Cancer Congress (EMCCC);

6. European Federation for Colorectal Cancer (EFR); and

7. European Cancer Congress (ECC).

We contacted individuals or organisations for information on unpublished or ongoing studies.

\section{Data collection and analysis}

\section{Selection of studies}

We imported all records retrieved from the searches into EndNote and removed duplicates (Endnote 2016). We exported these records to covidence for screening (Covidence 2018). Two review authors (MMG and MAT) independently examined the studies identified in the literature search and screened all studies based on their titles and abstracts, excluding studies that obviously did not meet the eligibility criteria. We did not exclude studies solely on the basis of reporting outcome data. We obtained the full texts of potentially eligible studies and the two review authors (MMG and MAT) independently examined the studies. In covidence, authors coded the studies as 'include', 'exclude' or 'uncertain' based on the outlined criteria. We resolved any disagreements through discussion, where necessary involving a third review author (CC or MMC), and kept a record of decisions made. We recorded reasons for exclusion of full text articles.

\section{Data extraction and management}

Two review authors (MMG and MAT) independently extracted data from the studies that met the inclusion criteria. We recorded extracted data on an excel spreadsheet, predeveloped for this purpose. MMG and MAT piloted the data extraction form in a random sample of three studies to ensure it captured the required information. We revised the form as required. We resolved any disagreements through discussion, and where necessary referred to a third review author (CC or MMC). Extracted data were entered into the Cochrane software Review Manager 5 (RevMan 5) (Review Manager 2014) for analyses. We extracted the following data.

1. Study details; author and year of publication, country of origin, aim, design, funding source, method of randomisation, method of recruitment, trial inclusion and exclusion criteria, duration of participation, conflicts of interest/ethical concerns, risk of bias assessment.

2. Participant details; total number randomised, age, gender, comorbidities, other relevant sociodemographics, cancer stage, type of cancer treatment, ethnicity, time since diagnosis, time beyond active treatment, baseline imbalances.

3. Intervention details; exercise type, intensity, frequency, volume, setting, duration of intervention, supervised or self-directed, details of control/comparison intervention, withdrawals and exclusion and co-interventions.

4. Outcomes; primary and secondary relevant to this review, including adverse events, follow-up time points, measurement tools used for outcomes.

\section{Assessment of risk of bias in included studies}

Two review authors (MMG and MAT) independently assessed each included study for risk of bias using the Cochrane 'Risk of bias' tool (Cochrane Handbook for Systematic Reviews of Interventions, Chapter 8.5.d, Higgins 2011a; Higgins 2017; Appendix 4). We assessed random sequence generation, allocation concealment, blinding of personnel and outcome assessment, completeness of outcome data, selective outcome reporting, and any other sources of bias, and judged the risk of bias as 'high', 'low' or 'unclear'. We resolved any disagreements through discussion and where necessary, through involving a third review author (CC or MMC). For each study, we detailed the risk of bias in table form alongside a statement of justification for our judgement. We summarised results in both a 'Risk of bias' summary figure and 'Risk of bias' graph. When interpreting treatment effects, we took into account the risk of bias for studies that contribute to that outcome.

\section{Measures of treatment effect}

For continuous outcomes (physical function, disease-related mental health, physical fitness, cancer-related fatigue, anthropometric measurements, levels of physical activity and $\mathrm{HRQ}$ oL) we determined the mean differences (MDs) or standardised

Physical activity interventions for disease-related physical and mental health during and following treatment in people with non- 
mean differences (SMDs) (in cases where different instruments were used to measure the selected outcome), in the intervention group compared with the control with 95\% confidence intervals (Cls). We extracted data for final scores and change from baseline scores, if available.

In this version of the review, no outcomes were reported as time-to-event and we were unable to report adverse events as a dichotomous outcome. In future versions of this review for timeto-event outcomes (overall survival and recurrence-free survival) we will extract hazard ratios (HRs) with standard errors, assuming that the HR is constant over time to compare the risk of death or recurrence of cancer in the treatment group with that in the control group. Where HRs are not presented, we will estimate them from reported data (e.g. Kaplan-Meier curves, logrank observed minus expected events and the logrank variance) using methods described by Tierney and colleagues (Tierney 2007). For adverse events, we will calculate the risk ratio (RR) at individual study level by dividing the risk of an event in the intervention group by the risk of the event in the control group. We will define RRs greater than 1.0 as favouring the control group (i.e. fewer adverse events in the control group) and RRs less than 1.0 as favouring the intervention group (Deeks 2017).

\section{Unit of analysis issues}

For parallel-group, individually randomised trials, the colorectal cancer participant was the unit of analysis in each study. No clusterRCTs met our inclusion criteria.

For studies reporting multiple follow-up time points, we conducted separate meta-analyses to reflect immediate-, short-, medium- and long-term periods of follow-up. For immediate-term follow-up we extracted data closest to the 12-week follow-up time point. For short- and medium-term follow-up, we extracted data closest to the six- and 12-month follow-up time point. For long-term follow-up, we extracted the longest time interval.

For studies with multiple arms, we included only relevant intervention arms. We combined all relevant intervention arms into a single group and combined all relevant control arms into a single group, creating a single, pair-wise comparison.

For future versions of this review, we will extract data from clusterRCTs when they report appropriate analyses, adjusting for the sample size in each cluster. Where control of clustering has not been performed we will attempt to correct for the intervention effects of cluster-RCTs by reducing the size of each study to its 'effective sample size', which is the number of the original sample size divided by the 'design effect'. We will calculate the design effect as $1+(M-1)^{*}$ ICC, where $M$ is the average cluster size and ICC is the intracluster correlation coefficient as described in the Cochrane Handbook for Systematic reviews of interventions, section 16.3.4 (Higgins 2011b). We will use an estimate of the ICC derived from the study (if possible), from a similar study or from a study of a similar population. If we use ICCs from other sources, we will report this and conduct sensitivity analyses to investigate the effect of variation in the ICC. If we identify both cluster-randomised trials and individually-randomised trials, we plan to synthesise the relevant information. We will consider it reasonable to combine the results from both if there is little heterogeneity between the study designs and the interaction between the effect of intervention and the choice of randomisation unit is considered unlikely.

\section{Dealing with missing data}

We attempted to contact authors of the included studies to request missing data on outcomes, participants and summary data via email. We documented reasons for missing data (missing at random or missing not at random) and how they were addressed. We assessed the extent to which studies analysed data according to the intention-to-treat principle. We assessed the level of missing data for included studies by comparing the number of participants included in the final analysis with the proportion of all participants in each study available in Characteristics of included studies. In future versions of this review, for studies at high risk of attrition bias, we will attempt to perform both the best-case and worst-case sensitivity analyses to assess the impact of missing data on the estimates of effect.

\section{Assessment of heterogeneity}

We evaluated clinical heterogeneity by examining diversity in participant characteristics, physical activity intervention characteristics, colorectal cancer treatment and outcomes among studies. We evaluated methodological heterogeneity by examining diversity in study designs and risk of bias. We did not pool methodologically heterogeneous studies. We visually inspected forest plots and used the $\mathrm{Chi} 2$ test to assess statistical heterogeneity (with $\mathrm{P}<0.1$ ). We used the $\mathrm{I}^{2}$ statistic to assess the percentage of variation across studies that is due to heterogeneity and not due to chance (Higgins 2003). We tentatively regarded heterogeneity as 'low' if 12 is less than $49 \%$, 'moderate', if 12 is between $50 \%$ and $75 \%$ and 'high' if $\mathrm{R} 2$ is more than 75\% (Deeks 2017). We investigated potential sources of statistical heterogeneity by reassessing diversity in characteristics of studies (participant, intervention, treatment and outcomes) and by means of sensitivity analysis.

\section{Assessment of reporting biases}

We attempted to control for time-lag bias, location bias, citation bias and language bias by using a comprehensive search strategy without language or date restriction, that included searching for unpublished studies and searching trials registers. We controlled for multiple publication bias by identifying duplicate publications of the same study and grouping these together, listing them as one study. For studies published after 1 July 2005, we screened the Clinical Trials Register at the WHO ICTRP for the study protocols (apps.who.int/trialsearch) to evaluate whether selective reporting of outcomes was present (outcome reporting bias).

No analysis in this version of the review included more than 10 studies. For future versions of this review, if there are at least 10 studies included in a meta-analysis, we will visually inspect funnel plots for asymmetry to investigate potential publication bias or small-study effects following the recommendations in Chapter 10 of the Cochrane Handbook for Systematic Reviews of Interventions for any statistical testing of funnel plot asymmetry (Sterne 2017).

\section{Data synthesis}

We pooled results from comparable groups of studies using both fixed-effect and random-effects models, when appropriate. Whenever possible, we used a random-effects model with inverse variance weighting for meta-analyses (DerSimonian 1986), due to the nature of physical activity as a highly varied intervention. We used a fixed-effect model when there were few studies or if 
the studies were small with few events. Where appropriate, we conducted a sensitivity analysis to investigate the effect of the choice of model (fixed-effect or random-effects) on the pooled estimate. MMG and CC conducted statistical analysis using RevMan 5 (Review Manager 2014). We considered a two-sided $P$ value of less than 0.05 as statistically significant. In cases where measurement tools for outcomes had the opposite direction of effect, we multiplied mean scores of a selected measurement by minus one to ensure all scales had the same direction of effect as discussed in the Cochrane Handbook for Systematic Reviews of Interventions section, 9.2.3.2 (Deeks 2017). Where data aggregation was not possible due to heterogeneity, we provided a narrative synthesis of study results. We summarised the findings of the systematic review alongside an assessment of the quality of evidence for each individual outcome using the GRADE approach (GRADE Working Group 2004).

\section{Subgroup analysis and investigation of heterogeneity}

No subgroup analyses were conducted in this version of the review due to insufficient data or inclusion of a range of treatment stages, treatment types, participant ages, gender in each individual study, or both. In future versions of this review where there are sufficient data, we will perform subgroup analyses of the effect of the intervention according to:

1. exercise and physical activity intervention characteristics (using frequency, intensity, time, type, volume progression (FITT-VP ) to calculate metabolic equivalents/hours per week);

2. participant characteristics (gender, age - over 65 years or under 65 years);

3. cancer stage (T1-2, N0, M0), (T3-4, N0, M0), (T1-4, N1-2, M0);

4. cancer type (colon or rectal);

5. treatment stage (during or post-treatment);

6. treatment type (laparoscopic or open surgery, neoadjuvant therapy or no neoadjuvant therapy);

7. time since diagnosis (zero to one year, two to three years, four to five years).

\section{Sensitivity analysis}

We undertook sensitivity analyses to assess the robustness of results. We reanalysed data after excluding studies with high risk of bias, those that had co-interventions (when appropriate) and studies that had not performed an intention-to-treat analysis. We conducted a sensitivity analysis to investigate heterogeneous results with the identification and removal of heterogeneous studies. We conducted a sensitivity analysis to investigate the effect of the choice of model (fixed-effect or random-effects) on the pooled estimate. In future versions of this review, for studies at high risk of attrition bias, we will conduct a best-case/worst-case sensitivity analysis to assess the impact of missing data on the estimates of effect. If there are any assumptions for ICC value used in cluster-RCTs, we will perform a sensitivity analysis.

\section{Summary of findings}

We assessed the overall quality of evidence of the main review outcomes using the GRADE approach in 'Summary of findings 1 (GRADE Working Group 2004). The 'Summary of findings' table highlights the overall quality of the body of evidence for the main review outcomes, using the GRADE criteria (study limitations (i.e. risk of bias), consistency of effect, indirectness, imprecision and publication bias). We used GRADEpro GDT 2015 software to prepare the 'Summary of findings' table. We will also present the results from the prespecified Sensitivity analysis and Subgroup analysis and investigation of heterogeneity when appropriate in 'Summary of findings' tables.

\section{RE S U L T S}

\section{Description of studies}

\section{Results of the search}

See: Characteristics of included studies; Characteristics of excluded studies; Characteristics of studies awaiting classification; Characteristics of ongoing studies

We conducted the comprehensive database search between 6 June 2019 and 14 June 2019 and found 5061 records. We identified 1092 additional records upon searching clinical trial registries and handsearching references lists, conference and meeting abstracts. After removing duplicates, 3837 potential records remained. We excluded a total of 3640 records based on the title and abstract and retrieved 197 records for more detailed evaluation (Figure 1). 
Figure 1. PRISMA flow diagram.

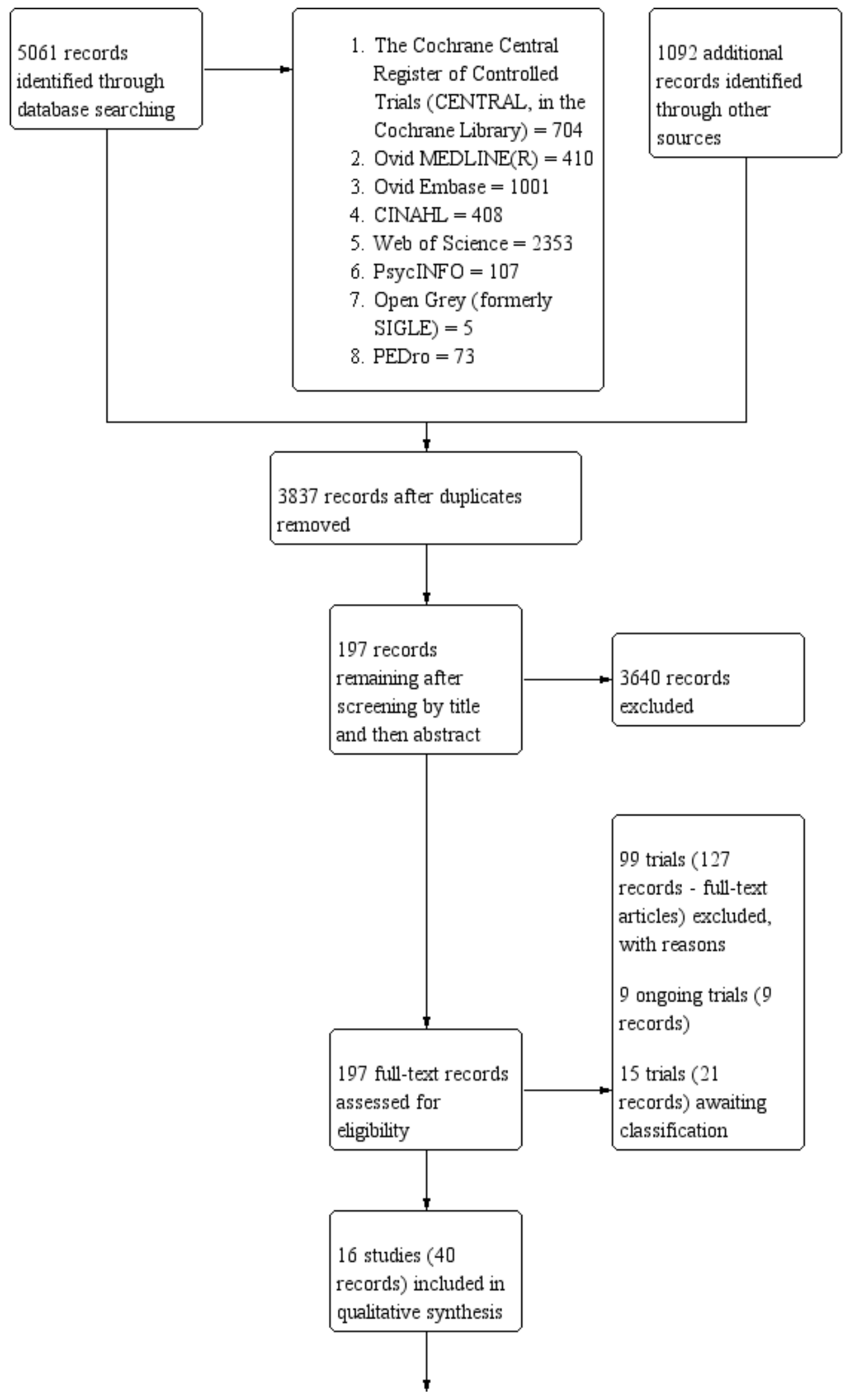

Physical activity interventions for disease-related physical and mental health during and following treatment in people with non- 
Figure 1. (Continued)

\begin{tabular}{l|}
16 studies (38 \\
records) included in \\
quantitative \\
synthesis \\
(meta-analysis)
\end{tabular}

From these, we excluded 99 studies (127 records) as they did not meet the inclusion criteria (see 'Characteristics of excluded studies') and 16 studies (40 records) were appropriate for inclusion in the current review. In addition, nine studies are ongoing and 15 studies (21 records) are awaiting classification; we did not include these studies in the analysis presented below, but will consider them in future updates of this review. We completed all searches by 14 June 2019. Figure 1 illustrates the process of the literature search and study selection for the review based on the PRISMA template (Moher 2009).

\section{Included studies}

We included 16 studies in this review (14 journal articles and 2 dissertations) (Bourke 2011; Brown 2017; Cantarero-Villanueva 2016; Courneya 2003; Courneya 2016; Cramer 2016; Hubbard 2016; Kim 2018; Lee 2017; Lewis 2016; McDermott 2017; Nuri 2016; Pinto 2013; Van Blarigan 2019; Van Vulpen 2016; Waart 2017). We used the main publication as the study reference. We reviewed and included information on study characteristics and outcome related data from an additional 28 publications that were secondary publications of seven of these 16 studies. We contacted 11 study authors for additional information (Bourke 2011; Brown 2017; Cantarero-Villanueva 2016; Courneya 2003; Cramer 2016; Lee 2017; Lewis 2016; McDermott 2017; Nuri 2016; Van Vulpen 2016; Waart 2017), eight of these authors replied to information requests (Bourke 2011; Courneya 2003; Cramer 2016; Lewis 2016; McDermott 2017; Nuri 2016; Van Vulpen 2016; Waart 2017). For study characteristics and outcomes, see the Characteristics of included studies table.

\section{Study characteristics}

All 16 included studies were randomised controlled trials (RCTs). No cluster-RCTs met our inclusion criteria. All studies except for two randomised participants to either a physical activity or usual care arm (Brown 2017; Waart 2017). These two studies included an additional study arm, that included variations in exercise volume in Brown 2017 and exercise intensity in Waart 2017. Three studies included co-interventions (Bourke 2011; Courneya 2016; Hubbard 2016), involving healthy eating seminars and a dietary information pack (Bourke 2011), health education materials for the usual care group (Courneya 2016), and weekly education sessions on topics, including physical activity, diet stress management and cardiac specific issues (Hubbard 2016). In all, investigators allocated 992 participants (mean 62, range 18 to 273 ) to a physical activity intervention group ( $n=524$, mean 33 , range 9 to 136 ) or a control group ( $n=468$, mean 29 , range 9 to 137 ).

\section{Participants}

Participants enrolled in the studies had a diagnosis of colon or colorectal cancer, six studies included only participants with colon cancer (Bourke 2011; Brown 2017; Cantarero-Villanueva 2016; Courneya 2016; Van Vulpen 2016; Waart 2017), whilst the remaining 10 studies included participants with colorectal cancer (Courneya 2003; Cramer 2016; Hubbard 2016; Kim 2018; Lee 2017; Lewis 2016; McDermott 2017; Nuri 2016; Pinto 2013; Van Blarigan 2019). Four studies reported the percentage of rectal cancer participants included (Kim 2018; McDermott 2017; Pinto 2013; Van Blarigan 2019). No studies with exclusively rectal cancer participants met our inclusion criteria. The majority of studies included participants with stage I-III colorectal cancer, however Courneya 2016, Lee 2017 and Waart 2017 excluded participants with stage I cancer. In addition, Waart 2017, Courneya 2003 and Van Blarigan 2019 included a minority of participants with stage IV cancer; when contacted Waart 2017 confirmed that no stage IV participants were included, Courneya 2003 was unable to provide separate data, excluding the four stage IV participants that were included in the study. Van Blarigan 2019 included one stage IV participant (2\% of total participants included). Ten studies included participants who had finished active treatment (Bourke 2011; Brown 2017; Cantarero-Villanueva 2016; Courneya 2016; Kim 2018; Lee 2017; Lewis 2016; McDermott 2017; Pinto 2013; Van Blarigan 2019), the time beyond treatment ranged between two months and five years. Two studies included participants receiving active treatment (Van Vulpen 2016; Waart 2017). Two studies were conducted among participants who were receiving and finished active treatment (Cramer 2016; Hubbard 2016). It was unclear whether participants were receiving or finished treatment in two studies (Courneya 2003; Nuri 2016). The majority of participants had undergone surgery as treatment; chemotherapy was also common across studies with less participants receiving radiotherapy. Mean time since diagnosis was only reported in six studies and ranged between 10 weeks and 2.99 years (Cantarero-Villanueva 2016; Courneya 2016; Lewis 2016; Pinto 2013; Van Blarigan 2019; Van Vulpen 2016).

The mean age of participants ranged between 51 and 69 years. Fifteen studies included both males and females, with one study including only males (Nuri 2016). Comorbidities and ethnicity were largely unreported in studies. Only two studies reported comorbidities at baseline (Brown 2017; Waart 2017). Two studies reported on ethnicity of participants (Brown 2017; Pinto 2013), in both studies the majority of participants were white. Eight studies reported on education levels of participants (CantareroVillanueva 2016; Courneya 2003; Cramer 2016; Kim 2018; Lee 2017; Van Blarigan 2019; Van Vulpen 2016; Waart 2017) with Kim 2018, Lee 2017, Van Vulpen 2016 and Waart 2017 further reporting on martial status and McDermott 2017, Van Blarigan 2019 and Waart 2017 
reporting on employment status. Five studies reported recruiting those who were currently inactive (Bourke 2011; Courneya 2016; Lee 2017; Lewis 2016; McDermott 2017).

\section{Interventions}

Type and setting of interventions varied across studies. Three studies opted for exclusively supervised physical activity interventions (Cantarero-Villanueva 2016; Cramer 2016; Hubbard 2016), likely due to the modes of exercise which included hatha yoga, core stabilisation exercise and cardiac rehabilitation exercise classes. The settings for these interventions were not clearly reported. Five studies opted for exclusively home-based selfdirected programmes (Brown 2017; Courneya 2003; McDermott 2017; Pinto 2013; Van Blarigan 2019), which consisted of mainly aerobic physical activity (e.g. treadmill walking, cycling). A combination of aerobic and resistance exercise was prescribed in two studies (McDermott 2017 ; Van Blarigan 2019). The Nuri 2016 study involved aerobic physical activity, whether the physical activity was supervised or self-directed is unclear. Seven studies opted for a combination of supervised and self-directed physical activity (Bourke 2011; Courneya 2016; Kim 2018; Lee 2017; Lewis 2016; Van Vulpen 2016; Waart 2017), with physical activity logs in Lee 2017 and telephone support in Courneya 2016 and Kim 2018. A combination of aerobic and resistance physical activity was conducted in four of these studies (Bourke 2011; Lee 2017; Van Vulpen 2016; Waart 2017), with Courneya 2016 encouraging activity based on individual preference, with a walking prescription if individuals had no preference.

The intensity of the physical activity varied slightly between studies. Methods used to measure intensity of the physical activity included relatively objective measures, such as percentage of maximum heart rate, heart rate at ventilatory threshold, percentage of predicted maximum workload and ratings of perceived exertion. The majority of studies opted for moderateintensity physical activity (Bourke 2011; Brown 2017; Courneya 2003; Lee 2017; Lewis 2016; McDermott 2017; Nuri 2016; Pinto 2013; Waart 2017), with three studies incorporating vigorous physical activity (Lewis 2016; Van Blarigan 2019; Waart 2017). An arm of the Waart 2017 study participated in low-intensity exercise. Five studies did not report intensity of the physical activity programme (Cantarero-Villanueva 2016; Courneya 2016; Cramer 2016; Hubbard 2016; Kim 2018).

The most common duration of physical activity intervention was 12 weeks (Bourke 2011; Kim 2018; Lee 2017; Lewis 2016; McDermott 2017; Pinto 2013; Van Blarigan 2019). In one study, the length of the intervention was determined by duration of chemotherapy, with participants beginning the intervention with the first cycle of chemotherapy and finishing three weeks after the last cycle (Waart 2017). For another study (Hubbard 2016), the length of intervention varied depending on hospital site $(6,10$ or 12 weeks). Two studies delivered eight-week interventions (Cantarero-Villanueva 2016; Nuri 2016). The duration of the other five studies were 10 weeks (Cramer 2016), 16 weeks (Courneya 2003), 18 weeks (Van Vulpen 2016), six months (Brown 2017), and three years (Courneya 2016). However, the Courneya 2016 study is ongoing and we have extracted one-year interim data for this review. All studies conducted follow-up assessments on completion of the exercise intervention. Nine studies conducted a further set of equivalent assessments at a later time point (Cantarero-Villanueva 2016; Cramer 2016; Hubbard 2016; Lewis 2016; McDermott 2017; Nuri
2016; Pinto 2013; Van Vulpen 2016; Waart 2017). Pinto 2013 was the only study to conduct three postintervention assessments, these took place at three, six and 12 months.

\section{Control groups}

The control groups received usual care, were not prescribed physical activity and did not take part in any formal exercise training during the course of the intervention. Five studies provided participants with written information on maintaining a healthy lifestyle (Courneya 2016; Hubbard 2016; Pinto 2013; Van Blarigan 2019), or provided recommendations for a healthy lifestyle (Cantarero-Villanueva 2016). Three studies reported asking participants in the control arm to maintain their usual daily physical activity levels/lifestyle habits during the intervention (Brown 2017; Lee 2017; Van Vulpen 2016). One study instructed participants not to initiate any structured exercise over the course of the intervention (Courneya 2003). Three studies had a waiting list control group, providing participants the opportunity to take part in the physical activity intervention following completion of the study (Brown 2017; Cramer 2016; Lewis 2016). Van Blarigan 2019 offered a fit bit flex to control group participants following study completion. Pinto 2013 controlled for frequency of contact with participants by having a contact control group.

\section{Outcome measures}

The most frequently assessed outcome among the 16 included studies was physical fitness, measured in 12 studies (Bourke 2011; Brown 2017; Cantarero-Villanueva 2016; Courneya 2003; Courneya 2016; Lee 2017; Lewis 2016; McDermott 2017; Nuri 2016; Pinto 2013; Van Vulpen 2016; Waart 2017), the most commonly used tools to measure physical fitness were the six-minute walk test (Brown 2017; Cantarero-Villanueva 2016; Courneya 2016; Lee 2017; Lewis 2016), and treadmill tests using a variety of protocols (Courneya 2003; Lewis 2016; Pinto 2013; Van Vulpen 2016; Waart 2017). Twelve studies measured levels of physical activity, objectively, using accelerometer data (Brown 2017; Hubbard 2016; Lewis 2016; McDermott 2017; Pinto 2013; Van Blarigan 2019), and subjectively, using a variety of self-report questionnaires (Bourke 2011; Courneya 2003; Courneya 2016; Kim 2018; Lee 2017; Lewis 2016; Pinto 2013; Waart 2017). Lewis 2016 and Pinto 2013 measured levels of physical activity, both objectively and subjectively. Ten studies assessed anthropometric measurements (Bourke 2011; Brown 2017; Cantarero-Villanueva 2016; Courneya 2003; Courneya 2016; Lee 2017; Lewis 2016; McDermott 2017; Nuri 2016; Van Vulpen 2016). All studies except for Courneya 2003 measured weight and only two did not measure body mass index (BMI) (Courneya 2016; Van Vulpen 2016). Fatigue and health-related quality of life (HRQoL) were assessed in nine studies, out of the 16 included (Bourke 2011; Courneya 2003; Cramer 2016; Kim 2018; Lewis 2016; McDermott 2017; Pinto 2013; Van Vulpen 2016; Waart 2017), most frequently using the Functional Assessment of Cancer Therapy - Fatigue (FACT-F) scale and Functional Assessment of Cancer Therapy - Colorectal (FACT-C) scale, respectively. Adverse events were reported in eight studies, of these the absence of any adverse events were reported in four studies (Hubbard 2016; Lewis 2016; Van Vulpen 2016; Waart 2017), whilst four studies recorded the number of adverse events that occurred (Brown 2017; CantareroVillanueva 2016; Cramer 2016; Van Blarigan 2019). Six studies assessed facets of mental health and well-being (Courneya 2003; Cramer 2016; Kim 2018; McDermott 2017; Van Vulpen 2016; Waart 2017), mainly anxiety and depression, using the Hospital Anxiety 
and Depression Scale (HADS) score. Other outcomes including overall and recurrence-free survival were not reported in any of the included studies. Adherence to Enhanced Recovery After Surgery (ERAS) guidelines and length of hospital stay was not reported in any of the included studies. For detailed information on outcome measures, see the Characteristics of included studies table.

\section{Excluded studies}

We excluded 99 trials from the review due to the following reasons.

1. Wrong patient population $(n=19)$

2. Wrong study design $(n=25)$

3. Wrong comparator $(n=18)$

4. Wrong intervention $(n=16)$

5. Intervention too short $(n=10)$

6. Did not analyse colorectal cancer patients separately $(n=8)$
7. Study was not carried out $(n=2)$

8. Outcomes were not relevant $(n=1)$

See Characteristics of excluded studies for an overview.

\section{Risk of bias in included studies}

We assessed the risk of bias for each included study using the 'Risk of bias' assessment tool and recommendations for judging risk of bias provided in Chapter 8 of the Cochrane Handbook for Systematic Reviews of Interventions (Higgins 2017). See Figure 2 for an overall assessment of risk of bias presented as percentages across all included studies. In addition, Figure 3 provides a 'Risk of bias' summary for each included study. Due to the nature of the intervention, it was expected that blinding of participants and personnel delivering the interventions would not be possible. We therefore judged risk of performance bias as high in all included studies. See Characteristics of included studies.

\section{Figure 2. Risk of bias graph: review authors' judgements about each risk of bias item presented as percentages across all included studies.}

Random sequence generation (selection bias)

Allocation concealment (selection bias)

Blinding of participants and personnel (performance bias): All outcomes

Blinding of outcome assessment (detection bias): All outcomes

Incomplete outcome data (attrition bias): All outcomes

Selective reporting (reporting bias)

Other bias

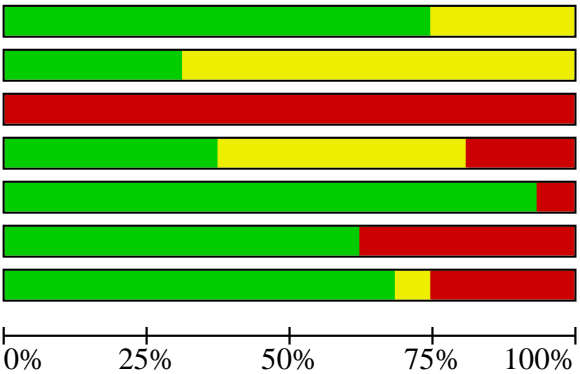

Low risk of bias

Unclear risk of bias

High risk of bias 
Figure 3. Risk of bias summary: review authors' judgements about each risk of bias item for each included study.

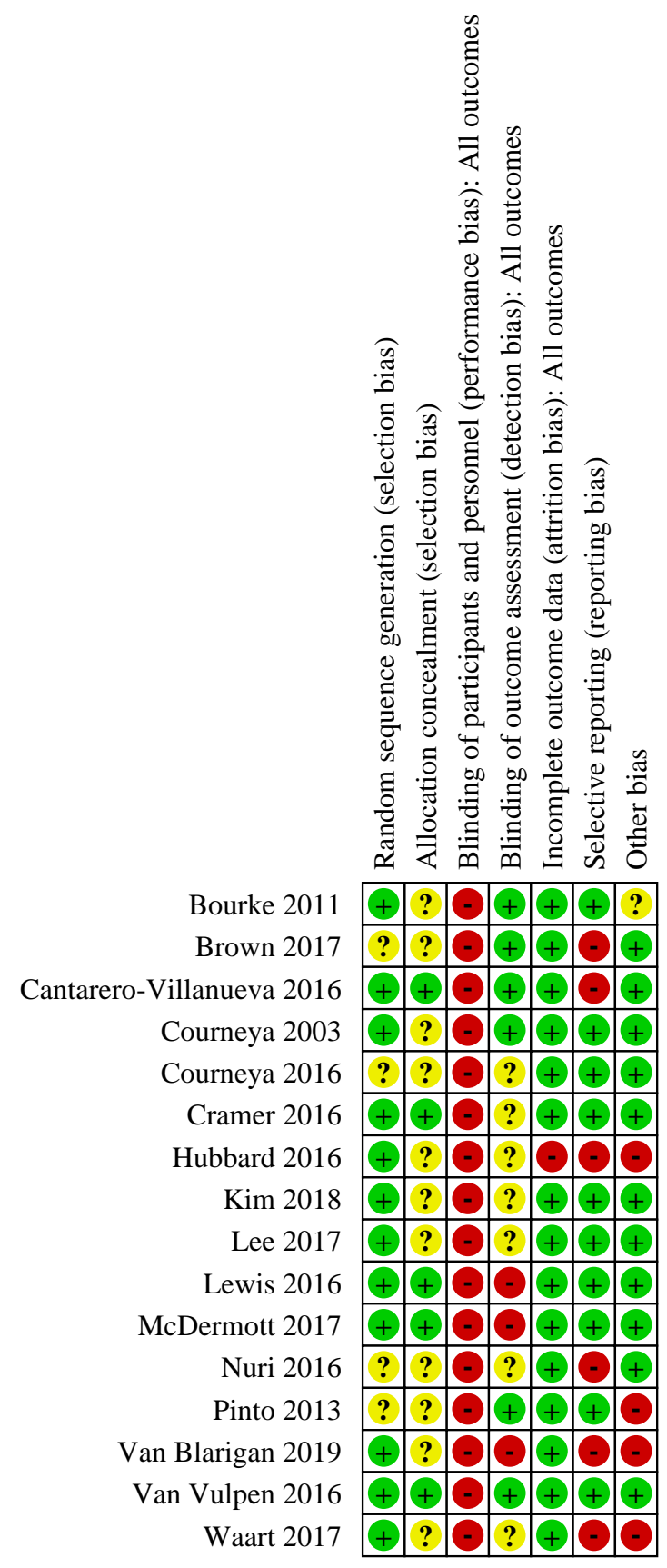

\section{Allocation}

\section{Random sequence generation}

Twelve studies $(75 \%)$ were at low risk of selection bias owing to adequate generation of a randomised sequence (Bourke 2011; Cantarero-Villanueva 2016; Courneya 2003; Cramer 2016; Hubbard 2016; Kim 2018; Lee 2017; Lewis 2016; McDermott 2017; Van Blarigan 2019; Van Vulpen 2016; Waart 2017). We considered four studies to have an unclear risk of selection bias as they did not describe the generation of a randomised sequence (Brown 2017; Courneya 2016; Nuri 2016; Pinto 2013).

\section{Allocation concealment}

Five studies (36\%) were at low risk of selection bias owing to adequate concealment of allocation, so that participants and investigators could not foresee assignment to the study groups

Physical activity interventions for disease-related physical and mental health during and following treatment in people with non- 
(Cantarero-Villanueva 2016; Cramer 2016; Lewis 2016; McDermott 2017; Van Vulpen 2016). The other 11 studies were considered to have an unclear risk of selection bias owing to allocation concealment as they did not describe the method of concealment.

\section{Blinding}

\section{Blinding of participants and personnel}

Due to the nature of physical activity interventions, blinding of participants and personnel is not possible. Therefore, we judged all included studies at high risk of performance bias.

\section{Blinding of outcome assessors}

Six studies were at low risk of detection bias as outcome assessors were blinded to participants group assignment (Bourke 2011; Brown 2017; Courneya 2003; Cantarero-Villanueva 2016; Pinto 2013; Van Vulpen 2016). We considered seven studies to have unclear risk for detection bias, as blinding of outcome assessors was not described (Courneya 2016; Cramer 2016; Hubbard 2016; Kim 2018; Lee 2017; Nuri 2016; Waart 2017). Three studies were at high risk for detection bias as the outcome assessor was aware of participants' group allocation (Lewis 2016; McDermott 2017; Van Blarigan 2019).

\section{Incomplete outcome data}

All included studies reported on adherence. All studies except for Hubbard 2016 were at low risk of attrition bias due to the amount, nature or handling of incomplete outcome data. Hubbard 2016 was at high risk of attrition bias due to the amount of missing data. Adherence to physical activity interventions in other studies varied between $71 \%$ and $97 \%$.

\section{Selective reporting}

Ten studies were at low risk of reporting bias (Bourke 2011; Courneya 2003; Courneya 2016; Cramer 2016; Kim 2018; Lee 2017; Lewis 2016; McDermott 2017; Pinto 2013; Van Vulpen 2016). Six studies were at high risk of reporting bias, as study protocols or methods sections included outcomes which were not reported in available publications (Brown 2017; Cantarero-Villanueva 2016; Hubbard 2016; Nuri 2016; Van Blarigan 2019; Waart 2017).

\section{Other potential sources of bias}

\section{Baseline imbalances}

Eleven studies were at low risk of selection bias owing to the absence of significant imbalances between group at baseline (Brown 2017; Cantarero-Villanueva 2016; Courneya 2003; Courneya 2016; Kim 2018; Lee 2017; Lewis 2016; Nuri 2016; Van Vulpen 2016), or in studies were baseline imbalances were present, appropriate allocation concealment was described (Cramer 2016; McDermott 2017). Four studies were at high risk of selection bias because group similarity at baseline was inadequate (Hubbard 2016; Pinto 2013; Van Blarigan 2019; Waart 2017). The risk of selection bias owing to baseline imbalances was unclear in one study as baseline imbalances were not reported (Bourke 2011). All included studies are at risk of participation bias, with the potential for the more motivated participants agreeing to participate.

\section{Effects of interventions}

See: Summary of findings 1 Physical activity compared with usual care in adults with non-advanced colorectal cancer
See: Summary of findings 1 . For a summary of sensitivity analyses see: Table 1.

All trial authors reported study results as follow-up values and six studies also included change in score from baseline to follow-up (Bourke 2011; Brown 2017; Cantarero-Villanueva 2016; Courneya 2016; Hubbard 2016; Lewis 2016). We completed meta-analyses for both types of outcomes separately and for each follow-up time period where data were available. We categorised followup as: up to 12 weeks after baseline (immediate); more than 12 weeks but less than or equal to six months after baseline (short term); more than six months but less than or equal to 12 months after baseline (medium term); and more than 12 months after baseline (long term). No included studies reported follow-up of greater than 12 months after baseline. One study reported followup time points to end of chemotherapy treatment and at six months following completion of chemotherapy treatment (Waart 2017). We calculated follow-up time points in months, using the reported percentage of chemotherapy treatment received. This was as a proportion of the average total planned duration of chemotherapy. Where studies had two intervention arms (Brown 2017; Waart 2017), or reported gender separately (Van Vulpen 2016), we combined these arms in RevMan to form a single pair-wise comparison (Review Manager 2014).

\section{Primary outcomes}

\section{Physical function}

A total of 10 studies reported on physical function, assessed using a variety of measures, including the 30-Second Chair Stand Test (Bourke 2011; Courneya 2016; Lee 2017; Lewis 2016; McDermott 2017), the Physical Functioning subscale of the Short Form-36 (SF-36) (Brown 2017; Pinto 2013), the functional wellbeing subscale of the Functional Assessment of Cancer TherapyColorectal (FACT-C; Courneya 2003), the physical function subscale of the European Organisation for Research and Treatment of Cancer Quality of life Questionnaire-Core 30 (EORTC QLQ-C30; Waart 2017), and the Trial Outcome Index-physical/functional/colorectal (Kim 2018). We conducted separate meta-analyses for objectively and subjectively measured physical function.

Four studies including 185 participants measured physical function using the 30-Second Chair Stand Test at immediate-term follow-up (Bourke 2011; Lee 2017; Lewis 2016; McDermott 2017), we did not pool these results in a meta-analysis due to considerable variation in results and inconsistency in direction of effect. Lewis 2016 and McDermott 2017 observed no difference between the physical activity and usual care groups for physical function at immediateterm follow-up, whilst, Bourke 2011 and Lee 2017 reported significant improvements in physical function in the physical activity group compared with usual care $(P=0.003$ and $P=0.005$, respectively). We observed no evidence of difference between groups at short-term follow-up when measured objectively (mean difference (MD) 0.76 , 95\% confidence interval (CI) -1.84 to 3.36 ; 2 studies, 39 participants; $1^{2}=0 \%$; moderate-quality evidence; Analysis 1.1; Figure 4), and subjectively at short-term follow-up (standardised mean difference (SMD) $0.09,95 \% \mathrm{Cl}-0.24$ to $0.42 ; 3$ studies, 156 participants; $I^{2}=0 \%$; low-quality evidence; Analysis 1.2; Figure 5). There were insufficient data to analyse subjective and objective physical function at medium-term follow-up, subjective physical function at immediate-term follow-up, and change from baseline results at all four follow-up time points.

Physical activity interventions for disease-related physical and mental health during and following treatment in people with non- 
Figure 4. Forest plot of comparison: 1 Physical activity versus usual care for physical function (30-sec chair sit-tostand test), outcome: 1.2 Objective measures more than 12 weeks to six months follow-up (short term).

\begin{tabular}{|c|c|c|c|c|c|c|c|c|c|}
\hline \multirow[b]{2}{*}{ Study or Subgroup } & \multicolumn{3}{|c|}{ Physical activity } & \multicolumn{3}{|c|}{ Usual care } & \multirow[b]{2}{*}{ Weight } & \multirow{2}{*}{$\begin{array}{l}\text { Mean Difference } \\
\text { IV, Fixed, 95\% CI }\end{array}$} & \multirow{2}{*}{$\begin{array}{l}\text { Mean Difference } \\
\text { IV, Fixed, 95\% CI }\end{array}$} \\
\hline & Mean & SD & Total & Mean & SD & Total & & & \\
\hline Lewis 2016 & 14 & 4.1 & 9 & 14.1 & 3.5 & 11 & $58.9 \%$ & $-0.10[-3.48,3.28]$ & \\
\hline McDermott 2017 & 18 & 5 & 10 & 16 & 4 & 9 & $41.1 \%$ & $2.00[-2.05,6.05]$ & \\
\hline Total $(95 \%$ CI $)$ & & & 19 & & & 20 & $100.0 \%$ & $0.76[-1.84,3.36]$ & \\
\hline \multicolumn{10}{|c|}{ Heterogeneity: $\mathrm{Chi}^{2}=0.61, \mathrm{df}=1(\mathrm{P}=0.44) ; \mathrm{I}^{2}=0 \%$} \\
\hline \multicolumn{9}{|c|}{ Test for overall effect: $\mathrm{Z}=0.58(\mathrm{P}=0.57)$} & $-20-10$ \\
\hline \multicolumn{9}{|c|}{ Test for subgroup differences: Not applicable } & Usual care \\
\hline
\end{tabular}

Figure 5. Forest plot of comparison: 1 Physical activity versus usual care for physical function, outcome: 1.3 Subjective measures more than 12 weeks to six months follow-up (short term).

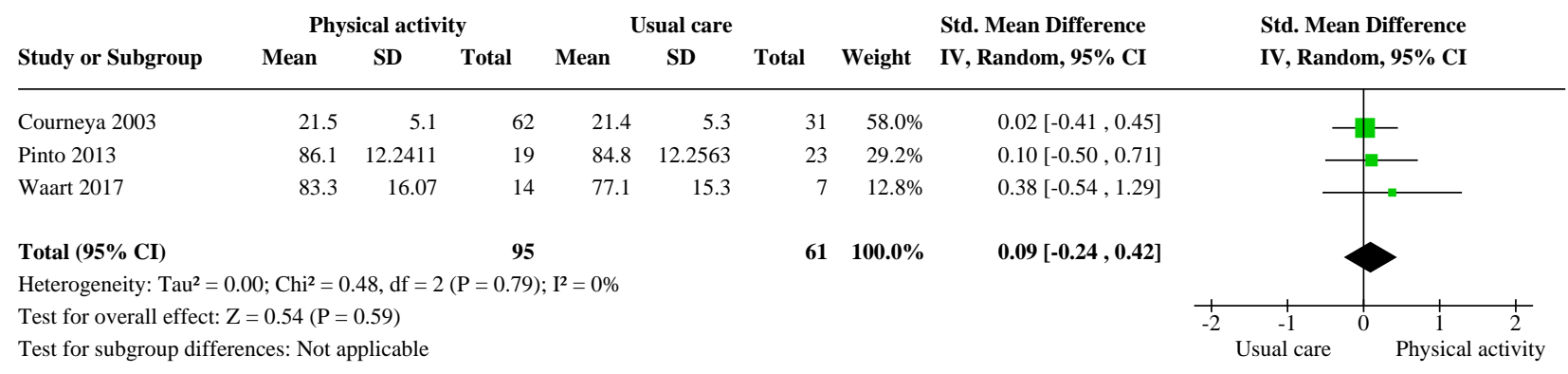

Courneya 2016 reported that the structured exercise group $(n=99)$ improved relative to the health education material group $(n=98)$ for the 30-Second Chair Stand Test (mean between group difference $(+1.6$ repetitions, $95 \% \mathrm{Cl}+0.6$ to +2.7$) ; \mathrm{P}<0.001)$ at one year. Pinto 2013 reported no significant group differences at immediate- or medium-term follow-up for self-reported physical functioning $(\mathrm{n}=$ 23 and $n=19$ for physical activity and usual care, respectively). Brown 2017 reported improvements in change from baseline scores for physical function at short-term follow-up with higher doses of physical activity resulting in greater improvements. Kim 2018 reported no difference between groups at immediate-term followup for physical function $(P=0.254)$. However, change from baseline to immediate-term follow-up improved in the intervention group (mean 64.1 (11.2) versus $66.3(11.8), \mathrm{P}=0.035)$.

We performed sensitivity analyses to investigate the choice of model on the pooled estimate at all included time points. Sensitivity analyses using a fixed-effect model were consistent with findings from a random-effects model. The sensitivity analysis revealed no difference in effect when we removed studies that did not use an intention-to-treat (ITT) analysis; this was only possible for subjective measures at short-term follow-up (Table 1 ).

\section{Disease-related mental health}

A total of seven studies reported on disease-related mental health, assessed by the Hospital Anxiety and Depression Scale (HADS) (Cramer 2016; Van Vulpen 2016; Waart 2017), the Positive and Negative Affect Scale (PANAS) (McDermott 2017), the State-Trait Anxiety Inventory (Courneya 2003), the Centre for Epidemiological Studies Depression Scale (CES-D) (Courneya 2003), and the Patient Health Questionnaire (Kim 2018). We obtained postintervention data and separate scores for anxiety and depression through email correspondence with authors Van Vulpen 2016 and Waart 2017 , respectively. We conducted meta-analyses for anxiety and depression separately at 12 weeks to six months after baseline and more than six months to 12 months after baseline.

There was no evidence of difference in depression between the physical activity group and usual care group at short-term followup (SMD - $0.21,95 \% \mathrm{Cl}-0.50$ to $0.08 ; 4$ studies, 198 participants; $12=$ $0 \%$; moderate-quality evidence; Analysis 2.1; Figure 6), or mediumterm follow-up (assessed using HADS) (MD $-1.20,95 \% \mathrm{Cl}-2.72$ to $0.31 ; 2$ studies, 48 participants; $1^{2}=32 \%$; low-quality evidence; Analysis 2.2; Figure 7). 
Figure 6. Forest plot of comparison: 2 Physical activity versus usual care for disease-related mental health, outcome: depression 2.1 More than 12 weeks to six months follow-up (short term).

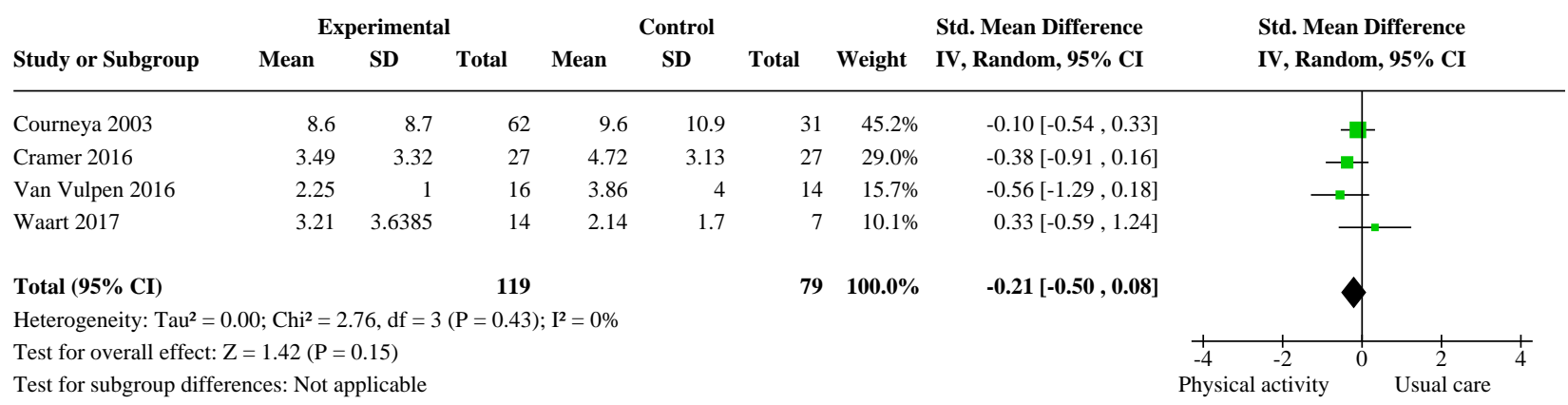

Figure 7. Forest plot of comparison: 2 Physical activity versus usual care for disease-related mental health (Hospital Anxiety and Depression Scale (HADS)), outcome: depression 2.2 More than six months to 12 months follow-up (medium term).

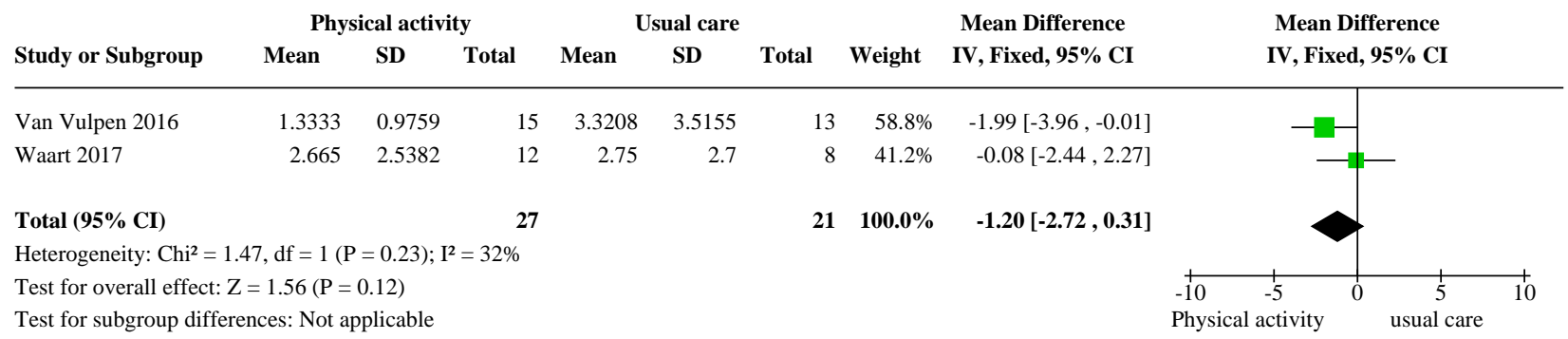

There was no evidence of difference in anxiety between the physical activity group and usual care group at short-term follow-up (SMD $-0.11,95 \% \mathrm{Cl}-0.40$ to 0.18 ; 4 studies, 198 participants; $\mathrm{I}^{2}=0 \%$; moderate-quality evidence; Analysis 2.3; Figure 8), or mediumterm follow-up (assessed using HADS) (anxiety: MD 1.79, 95\% Cl
-0.37 to $3.94 ; 2$ studies, 47 participants; $1^{2}=30 \%$; low-quality evidence; Analysis 2.4; Figure 9). Data were insufficient for analysis of immediate-term follow-up and change scores at all four time points.

Figure 8. Forest plot of comparison: 2 Physical activity versus usual care for disease-related mental health, outcome: 2.3 Anxiety more than 12 weeks to six months follow-up.

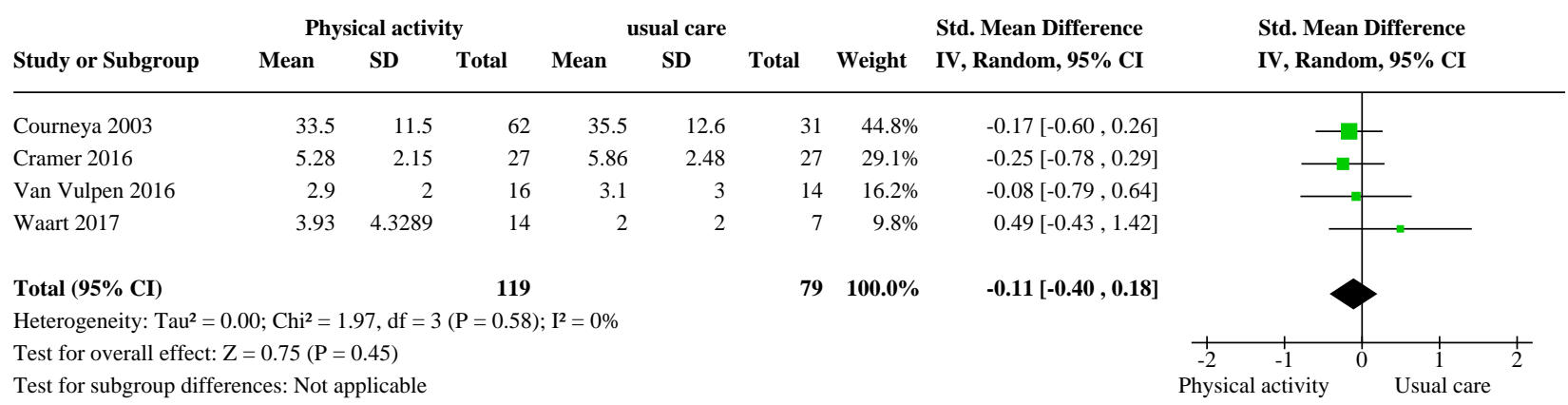


Figure 9. Forest plot of comparison: 2 Physical activity versus usual care for disease-related mental health (Hospital Anxiety and Depression Scale (HADS)), outcome: 2.4 Anxiety more than six months to 12 months follow-up (medium term).

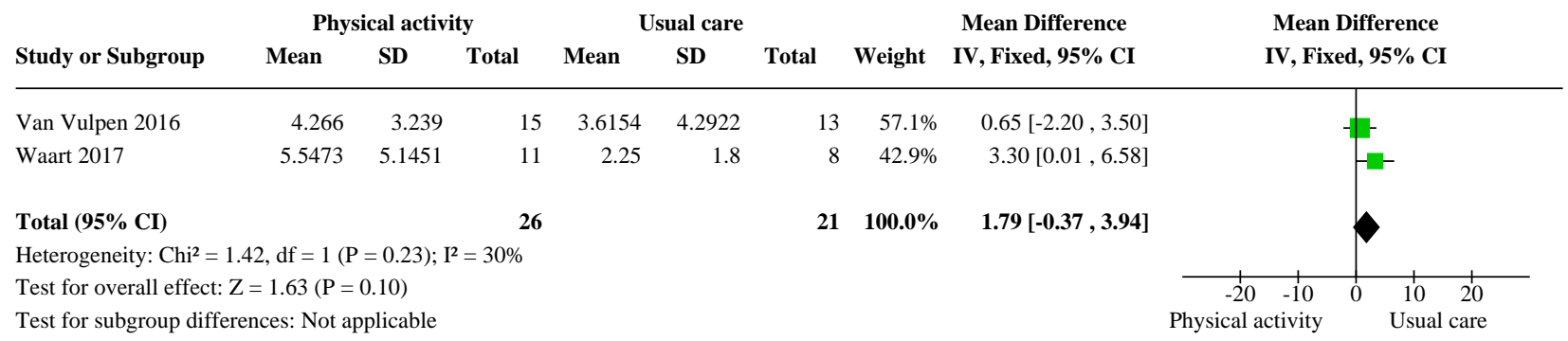

McDermott 2017 (unpublished data) reported no statistically significant differences between intervention $(n=11)$ and control $(\mathrm{n}=9)$ for PANAS-positive affect and PANAS-negative affect at immediate- or short-term follow-up. There were 10 participants in the control group at 24 weeks follow-up for PANAS-negative affect. Kim 2018 reported improvements in change from baseline scores for depression at immediate-term follow-up in the intervention group $(n=37)(P=0.053)$.

A sensitivity analysis including studies at low risk of bias and those that performed an ITT analysis suggest there may be no effect of physical activity on anxiety (SMD $-0.29,95 \% \mathrm{Cl}-0.60$ to $0.01 ; 3$ studies, 177 participants; $\mathrm{I}^{2}=0 \%$ ) or depression (SMD $-0.18, \mathrm{Cl}-0.48$ to 0.13 ; 3 studies, 177 participants; $12=0 \%$ ) in the physical activity group compared to usual care at short-term follow-up (Table 1 ).

\section{Adverse events}

Eight out of the 16 included studies reported on adverse events (Brown 2017; Cantarero-Villanueva 2016; Cramer 2016; Hubbard 2016; Lewis 2016; Van Blarigan 2019; Van Vulpen 2016; Waart 2017). We did not pool adverse events due to inconsistency in reporting and measurement. Of the eight studies, four studies reported that no adverse events (Hubbard 2016; Lewis 2016; Waart 2017) or serious adverse events (Van Vulpen 2016) occurred during the study period. The method of measurement of adverse events was not described by authors, Van Vulpen 2016 and Waart 2017. Van Vulpen 2016 and Lewis 2016 did not make reference to 'nonserious' adverse events. Hubbard 2016 recorded adverse events in a participant log and recorded them as "related" or "unrelated" to the study. In the study by Lewis 2016, serious adverse events were recorded by the researcher within 24 hours of becoming aware of the event.

Cramer 2016 recorded all adverse events that occurred during the study period and asked open-ended questions at weeks 10 and 22 to assess any adverse events not previously mentioned. No serious adverse events occurred and seven participants reported minor adverse events in the intervention group, including transient abdominal pain $(n=1)$, muscle soreness $(n=3)$, neck pain $(n=1)$, minor vertigo $(n=1)$ and hip pain $(n=1)$. One patient in the control group experienced a serious adverse event that was reported as "probably not causally related to the study intervention". Brown 2017 assessed adverse events using the Common Terminology Criteria for Assessing Adverse Events (CTCAE). No serious (grade 3) adverse events were reported at six months after baseline. One hundred and one non-serious (grade 1 and 2) adverse events occurred in the intervention compared to 49 in the usual care group. Common non-serious adverse events reported in the intervention group were: joint pain, back pain, generalised flu-like symptoms, foot blisters and myalgia. In the study by Cantarero-Villanueva 2016 each participant kept a diary to record adverse events. Six participants expressed both neck and abdominal discomfort with some of the exercises in the first sessions. Two participants in the intervention group and one participant in the usual care group experienced postoperative ventral hernias. One participant could not perform the aerobic exercise during one week because he suffered a peripheral neuropathy. The author did not report whether these were recorded as "related" or "unrelated" to the exercise intervention. In the study by Van Blarigan 2019 participants completed an online health check survey which recorded adverse events. Commonly reported non-serious adverse events included low back, knee, joint, muscle and chest pain, inflammation of joints in both the intervention $(n=39)$ and control $(n=36)$ groups.

We graded the evidence of the included studies as low quality (8 studies, 305 participants).

\section{Secondary outcomes}

\section{Overall survival}

No RCTs reported overall survival.

\section{Recurrence-free survival}

No RCTs reported recurrence-free survival as an outcome. No studies reported cancer recurrence as reasons for dropout. One study reported lung metastasis in the intervention group as a reason for dropout (Lewis 2016).

\section{Physical fitness}

\section{Aerobic fitness}

A total of 12 studies reported aerobic fitness, using a variety of different measures including; the six-minute walk test (Brown 2017; Cantarero-Villanueva 2016; Courneya 2016; Lee 2017; McDermott 2017), the Bruce Protocol Treadmill Test (Bourke 2011; Lewis 2016), the Modified Balke Treadmill Test (Courneya 2003), $\mathrm{VO}_{2}$ peak test (Van Vulpen 2016); Cycle Ergometer Peak Power Output Test (Van Vulpen 2016), Rockport Walk Test (Nuri 2016), treadwalk test (Pinto 2013), and the Steep Ramp Test (Waart 2017). Nuri 2016 and Pinto 2013 estimated $\mathrm{V}_{2}$ peak from submaximal fitness tests; we used this data in our meta-analysis. No included studies reported change from baseline data at more than 12 months follow-up. 
At immediate-term follow-up, we observed an improvement in aerobic fitness in the physical activity group compared with the usual care group (SMD 0.82, 95\% Cl 0.34 to $1.29 ; 7$ studies, 295 participants; $1^{2}=68 \%$; low-quality evidence; Analysis 3.1). This effect was also observed at short-term follow-up (SMD 0.56, 95\% CI 0.29 to $0.82 ; 7$ studies, 248 participants; $1^{2}=1 \%$; moderate-quality evidence; Analysis 3.3), but not at medium-term follow-up (SMD $0.44,95 \% \mathrm{Cl}-0.04$ to 0.92 ; 4 studies, 272 participants; $\left.\right|^{2}=57 \%$; very low-quality evidence; Analysis 3.5).

Change in aerobic fitness from baseline showed an improvement compared with usual care at immediate-term follow-up (SMD 0.89, $95 \% \mathrm{Cl} 0.43$ to 1.36 ; 3 studies, 81 participants; $\mathrm{I}^{2}=0 \%$; low-quality evidence; Analysis 3.2) and short-term follow-up (SMD 0.62, 95\% Cl 0.05 to 1.19 ; 2 studies, 51 participants; $\left.\right|^{2}=0 \%$; moderate-quality evidence; Analysis 3.4). We were unable to include data from the study by Brown 2017 in the meta-analysis as the authors reported mean change between groups over time. We emailed to request postintervention and change from baseline data, but no reply was received.

We conducted a sensitivity analysis excluding studies with an additional component (dietary advice), studies that did not conduct an ITT analysis and those at high risk of bias; results suggest improvements in aerobic fitness in the physical activity group compared with usual care (SMD $0.38, \mathrm{Cl} 0.06$ to $0.70 ; 4$ studies, 207 participants; $12=15 \%$; Table 1) at immediate- term follow-up and short-term follow-up (SMD $0.45, \mathrm{Cl} 0.15$ to $0.75 ; 5$ studies, 187 participants; $\mathrm{I}^{2}=0 \%$; Table 1 ). We also conducted a sensitivity analysis at medium-term follow-up excluding the study in which the physical activity intervention was ongoing. Results of this suggest no improvement in aerobic fitness in the physical activity group compared with usual care (SMD 0.44, Cl 0.41 to 1.29; 3 studies, 86 participants).

\section{Upper body strength}

A total of five studies reported upper body strength, including arm strength, assessed by hand grip dynamometry (Lee 2017; Lewis 2016; Waart 2017), the 30 second arm-curl test (Lewis 2016; Courneya 2016), isometric abdominal strength and isometric back strength assessed by the trunk-curl test and back dynamometry, respectively (Cantarero-Villanueva 2016), and the push-up test to assess upper body strength and endurance (Lee 2017). A metaanalysis was conducted for hand grip strength only, due to the large variability in measurement of upper body strength. Data were insufficient for analysis of more than six months to 12 months follow-up and for change scores at all four time points.

We observed no difference in hand grip strength, assessed using hand grip dynamometry in the physical activity group compared with usual care at immediate-term follow-up (MD 1.92, 95\% Cl-1.17 to $5.00 ; 2$ studies, 147 participants; $\left.\right|^{2}=0 \%$, low-quality evidence; Analysis 4.1) or at short-term follow-up (MD $0.94,95 \% \mathrm{Cl}-5.98$ to 7.87; 2 studies, 39 participants; $12=0 \%$; very low-quality evidence; Analysis 4.2). In the study by Lee 2017 , hand grip strength was reported separately for the left and right hand. We contacted Lee 2017 to request combined data, but we received no response. We inputted the data from the right hand only.

Lee 2017 reported a significantly greater improvement in the pushup test $(P<0.001)$ in the intervention group $(n=62)$ compared with control $(n=61)$ at 12 weeks. The physical activity group $(n=21)$ experienced a greater increase in isometric abdominal strength compared with that of the usual care group $(n=19)$ at eight weeks $(P=0.001)$ but not at six months follow-up in the study by Cantarero-Villanueva 2016. No significant difference was found between groups for isometric back strength. There were no significant group differences in change in arm-curl repetitions in the study by Lewis 2016 ( $n=12$ for the physical activity group and usual care group at 12 weeks; $n=9$ and $n=11$ at 6 months, respectively) or in the study by Courneya $2016(P=0.18)(n=99$ and $n=98$ for the physical activity and usual care groups, respectively) at one year.

\section{Flexibility}

Three studies measured flexibility, assessed by the Sit and Reach Test (Courneya 2003; Courneya 2016), and the Modified Sit and Reach Test (Cantarero-Villanueva 2016). Cantarero-Villanueva 2016 reported flexibility of the right and left side separately. We emailed the author, but were unable to obtain combined sit and reach scores. We used data for the right side in our analysis. Mean scores were multiplied by minus one to ensure all scales had the same direction of effect as discussed in the Cochrane Handbook for Systematic Reviews of Interventions section, 9.2.3.2 (Deeks 2017). We observed no difference in flexibility in the physical activity group compared to usual care at short-term follow-up (SMD 0.02, 95\% $\mathrm{Cl}-0.36$ to $0.39 ; 2$ studies, 119 participants; $\mathrm{I}^{2}=0 \%$; low-quality evidence; Analysis 5.1). There were insufficient data for analysis of flexibility at immediate- and medium-term follow-up and for change scores at all four time points.

Courneya 2016 reported that the structured exercise group $(n=99)$ improved relative to the health education material group $(n=98)$ for the Sit and Reach Test (mean between group difference of +2.1 $\mathrm{cm} ; 95 \% \mathrm{Cl}-0.6$ to $+4.7 ; \mathrm{P}=0.08)$ at one year.

\section{Lower limb strength}

Two studies measured lower body strength, using dynamometry to assess maximum voluntary torque production of the knee extensors (Bourke 2011; Waart 2017). Meta-analysis was precluded as studies measured lower limb strength at different time points. In the study by Bourke 2011, no difference in maximum voluntary torque was observed between the intervention $(n=9)$ and control group $(n=9)$ over the 12-week intervention period ( $P=0.127$ ). Conversely, Waart 2017 observed improvements in lower body strength in the usual care control group $(n=6)$, the onco-move group (low-intensity home-based exercise; $n=6$ ) and a reduction in strength in the on-track intervention group (moderate- to highintensity supervised exercise; $n=7$ ) at the end of chemotherapy (calculated as 4.8 to 5 months follow- up). At six months following chemotherapy (calculated as 10 to 11 months follow-up), the ontrack group $(n=6)$ recovered to above baseline values (no $P$ values were reported).

\section{Cancer-related fatigue}

A total of 10 studies reported cancer-related fatigue, assessed by the Functional Assessment of Chronic Illness Therapy - Fatigue (FACIT-F) (Cramer 2016; Kim 2018; McDermott 2017), the Functional Assessment of Cancer Therapy - Fatigue (FACT-F) (Bourke 2011; Courneya 2003; Lewis 2016; Pinto 2013), the Multidimensional Fatigue Inventory (MFI) (Van Vulpen 2016; Waart 2017), the Multidimensional Fatigue Symptom Inventory - Short Form (MFSISF) (McDermott 2017), and the Fatigue Symptom Inventory (FSI) (Brown 2017). Van Vulpen 2016 and Waart 2017 reported results 
separately for subscales of the MFI. We extracted the MFI general subscale as a comparable measure for inclusion in the metaanalysis. We chose the general subscale score as it includes general statements about fatigue and decreased functioning, designed to encompass both physical and psychological aspects of fatigue (Lin 2009). We multiplied mean scores from the MFI general subscale by minus one to ensure all scales had the same direction of effect, as discussed in the Cochrane Handbook for Systematic Reviews of Interventions section, 9.2.3.2 (Deeks 2017).

Low-quality evidence suggests an improvement in cancer-related fatigue in the physical activity group compared with the usual care group at immediate-term follow-up (MD 2.16, 95\% Cl 0.18 to 4.15; 6 studies, 230 participants; $12=18 \%$; Analysis 6.1 ), short-term followup (SMD $0.34,95 \% \mathrm{Cl} 0.08$ to $0.60 ; 7$ studies, 277 participants; 12 = 9\%; moderate-quality evidence; Analysis 6.3), but not mediumterm follow-up (SMD $0.25,95 \% \mathrm{Cl}-0.16$ to $0.67 ; 3$ studies, 91 participants; $12=0 \%$; low-quality evidence; Analysis 6.4). Change from baseline to 12 weeks suggests no improvement in fatigue in the physical activity group compared with the usual care group (MD $0.41,95 \% \mathrm{Cl}-1.33$ to 2.14 ; 3 studies, 113 participants; $\left.\right|^{2}=70 \%$; low-quality evidence; Analysis 6.2). There were insufficient data to conduct change from baseline analysis at all other time points. Brown 2017 reported improvements in change from baseline scores in the high dose intervention group compared with control at shortterm follow-up ( $P$ trend $=0.045$ ).

We conducted a sensitivity analysis with studies at low risk of bias and those that performed an ITT analysis at immediate- and shortterm follow-up. There was no difference in fatigue between groups at immediate- or short-term follow-up (Table 1). Results from the fixed-effect sensitivity analysis were consistent with those from the random-effects model.

\section{Anthropometric measurements}

A total of 10 studies reported anthropometric measurements assessed by: weight (Bourke 2011; Cantarero-Villanueva 2016; Courneya 2016; Lee 2017; Lewis 2016; McDermott 2017; Nuri 2016; Van Vulpen 2016), BMI (Bourke 2011; Brown 2017; CantareroVillanueva 2016; Courneya 2003; Lee 2017; Lewis 2016; McDermott 2017; Nuri 2016), waist measurement (Brown 2017; CantareroVillanueva 2016; Lee 2017; McDermott 2017), waist-to-hip ratio (Bourke 2011; Brown 2017; Lewis 2016; McDermott 2017), body fat percentage (Cantarero-Villanueva 2016; Courneya 2003; Lee 2017; Lewis 2016; Nuri 2016), and hip circumference (Brown 2017; Courneya 2016; McDermott 2017). Brown 2017 also assessed visceral fat, subcutaneous fat and fat mass.

We found no evidence of effect of physical activity compared with usual care on weight (Analysis 7.1: moderate-quality evidence and Analysis 8.3: low-quality evidence), BMI (Analysis 11.1: lowquality evidence and Analysis 11.3: low-quality evidence), waist measurement (Analysis 8.1: very low-quality evidence and Analysis 8.2: low-quality evidence), waist-to -hip ratio (Analysis 9.1: very lowquality evidence and Analysis 9.3: low-quality evidence), or body fat percentage (Analysis 10.1: low-quality evidence and Analysis 10.3: very low-quality evidence) at immediate- or short-term follow-up time points.

Change from baseline to 12 weeks follow-up, but not change from baseline from 12 weeks to six months follow-up suggests a small reduction in weight (MD $-1.71,95 \% \mathrm{Cl}-2.90$ to $-0.51 ; 3$ studies, 82 participants; $12=1 \%$; low-quality evidence; Analysis 7.2; Analysis 7.3) and body fat percentage (MD $-1.57,95 \% \mathrm{Cl}-3.11$ to $-0.04 ; 2$ studies, 60 participants; $\left.\right|^{2}=0 \%$; low-quality evidence; Analysis 10.2 and Analysis 10.4), but not BMI at either time point (Analysis 11.2; Analysis 11.4; low- and very low-quality evidence, respectively, or change from baseline up to 12 weeks for waist-to-hip-ratio (Analysis 9.2; very low-quality evidence). Low-quality evidence suggests a small reduction in waist measurement in change from baseline from 12 weeks to six months follow-up (MD $-2.79,95 \% \mathrm{Cl}-5.21$ to -0.36 ; 2 studies, 70 participants; $I^{2}=38 \%$; Analysis 8.3$)$. When we conducted a sensitivity analysis using a random-effects model for waist measurement, the difference between the physical activity and usual care group was no longer significant (MD -4.36 cm, -11.42 to $2.71 ; 2$ studies, 70 participants; $12=38 \%$ ).

There was insufficient postintervention and change from baseline data at six months to 12 months to report on any anthropometric measure. Insufficient data precluded meta-analysis of change from baseline data to 12 weeks follow-up for waist measurement and more than 12 weeks to six months follow-up for waist-to-hipratio. We did not pool studies that measured hip circumference from 12 weeks to six months follow-up (Brown 2017; McDermott 2017), as Brown 2017 reported change from baseline scores whilst McDermott 2017 reported postintervention scores.

Courneya 2016 reported no statistically significant difference between the structured exercise programme (SEP) group and the health education materials (HEM) group for weight (SEP $=115$, HEM $=112)(P=0.38)$, hip circumference $(S E P=99, \mathrm{HEM}=99)(P=$ $0.90)$ or waist circumference $(\mathrm{SEP}=99, \mathrm{HEM}=99)(P=0.31)$ at one year. Between-group changes in body weight were not significantly different between intervention $(n=16)$ and usual care $(n=14)$ for both men and women in the study by Van Vulpen 2016 at 18 and 36 weeks. Changes in waist-to-hip ratio were not statistically significance in the study by Lewis $2016(P=0.43)$ or Brown 2017 ( $P$ $=0.054)$. Cantarero-Villanueva 2016 reported a significant change from baseline scores to eight weeks and six months between the physical activity group $(n=21)$ and usual care group $(n=19)$ for waist circumference. Changes in hip circumference up to six months were not significant in the study by Brown $2017(P=0.518)$, whilst McDermott 2017 reported an increase in hip circumference in the control group at week $12(n=9)$ and a decrease at week $24(n=$ 9), but not to pre-intervention levels, whilst the intervention group decreased at week $12(n=11)$ and increased at week $24(n=10)$ but not to pre-intervention levels $(P=0.012)$.

We conducted a sensitivity analysis for weight, waist-to-hip-ratio, BMI and body fat percentage at immediate-term follow-up, we excluded the study by Bourke 2011 because the intervention contained a dietary advice component and the study by Nuri 2016 as an ITT analysis was not conducted. Results of the sensitivity analysis suggests no difference in effect of physical activity compared with usual care on weight, BMI, waist measurement, waist-to-hip ratio or body fat percentage. The choice of model (fixed or random) did not effect the estimates of effect at this time point. A sensitivity analysis for change from baseline to 12 weeks follow-up suggested no reduction in weight or BMI when the study by Bourke 2011 was removed. A sensitivity analysis with studies at low risk of bias was conducted for weight and BMI for change scores from 12 weeks to six months, results suggest no difference in effect of physical activity compared with usual care on weight or BMI at this time point.

Physical activity interventions for disease-related physical and mental health during and following treatment in people with non- 


\section{Health-related quality of life (HRQOL)}

A total of 10 studies reported HRQoL; seven of these studies assessed HRQoL using the Functional Assessment of Cancer Therapy-Colorectal (FACT-C; Bourke 2011; Brown 2017; Courneya 2003; Cramer 2016; Kim 2018; McDermott 2017; Pinto 2013), the Functional Assessment of Cancer Therapy-General (FACT-G; Lewis 2016), the European Organisation for Research and Treatment of Cancer Quality of life Questionnaire-Core 30 (EORTC QLQ-C30; Van Vulpen 2016; Waart 2017), the EuroQol-Visual Analogue Scales (EQ-VAS; Lewis 2016), and the Satisfaction With Life Scale (SWLS; Courneya 2003).

Moderate-quality evidence suggests a small positive effect for the physical activity group compared with the usual care group on HRQoL at immediate-term follow-up (SMD $0.36,95 \% \mathrm{Cl} 0.10$ to 0.62 ; 6 studies, 230 participants; $\left.\right|^{2}=0 \%$; Analysis 12.1 ) and shortterm follow-up (SMD 0.45, 95\% Cl 0.03 to $0.88 ; 7$ studies, 278 participants; $\mathrm{I}^{2}=61 \%$; Analysis 12.3 ) but no evidence of difference between groups at medium-term follow-up (SMD 0.05, 95\% Cl-0.37 to $0.47 ; 3$ studies, 89 participants; $1^{2}=0 \%$; low-quality evidence; Analysis 12.5) or change from baseline up to 12 weeks follow-up (SMD $-0.10,95 \% \mathrm{Cl}-0.47$ to $0.28 ; 3$ studies, 113 participants; $\mathrm{I}^{2}=$ $16 \%$; low-quality evidence; Analysis 12.2). We observed a small positive effect on change from baseline scores at more than 12 weeks to six months follow-up (SMD $0.70,95 \% \mathrm{Cl} 0.14$ to 1.26 ; 2 studies, 58 participants; $1^{2}=0 \%$; low-quality evidence; Analysis 12.4). Data were insufficient to conduct a meta-analysis of change from baseline scores at more than six months to 12 months followup.

A sensitivity analysis suggests that physical activity interventions may have a positive effect on HRQoL compared to usual care at immediate-term follow-up, (excluding the study by Bourke 2011), and including studies at low risk of bias and those that conducted an ITT analysis (SMD $0.37,95 \% \mathrm{Cl} 0.07$ to $0.68 ; 4$ studies, 169 participants; 12 = 0\%; Table 1 ).

\section{Levels of physical activity}

A total of 12 studies reported on levels of physical activity, assessed objectively and subjectively. Objective physical activity was assessed by accelerometry (Brown 2017; Hubbard 2016; Lewis 2016; McDermott 2017; Van Blarigan 2019). We pooled total minutes of moderate to vigorous physical activity for the meta-analysis. Subjective physical activity was assessed by the Godin Leisure Time Exercise Questionnaire (Bourke 2011; Courneya 2003; Kim 2018; Lee 2017), the International Physical Activity Questionnaire (IPAQ; Lewis 2016), the Seven-Day Physical Activity Recall (7-day PAR) questionnaire (Courneya 2003; Pinto 2013), the Tartu Physical Activity Questionnaire (TPAQ; Courneya 2016), the Community Healthy Activities Model Programme for Seniors (CHAMPS; Courneya 2016), and the Physical Activity Scale for the Elderly (PASE; Waart 2017).

We found no evidence of difference in levels of physical activity in the physical activity group compared to usual care at immediateterm follow-up (MD $-8.34,95 \% \mathrm{Cl}-21.05$ to $4.37 ; 4$ studies, 94 participants; $\left.\right|^{2}=43 \%$; very low-quality evidence; Analysis 13.1 ) or short-term follow-up (MD 13.50, 95\% Cl -56.73 to 83.74; 2 studies, 36 participants; $I^{2}=13 \%$; Analysis 13.4 ) when measured objectively using accelerometry. We found no evidence of difference in change from baseline scores up to 12 weeks between groups (SMD - 0.13 ,
$95 \% \mathrm{Cl}-0.77$ to $0.52 ; 2$ studies, 37 participants; $\mathrm{I}^{2}=0 \%$; very lowquality evidence; Analysis 13.2).

Subjective measures of levels of physical activity suggest an increase in levels of physical activity in the physical activity group compared to the usual care group at immediate-term follow-up (SMD $0.70,95 \% \mathrm{Cl} 0.38$ to $1.03 ; 4$ studies, 156 participants; $\mathrm{I}^{2}=$ $0 \%$; very low-quality evidence; Analysis 13.3 ), short-term follow-up (SMD $0.39,95 \% \mathrm{Cl}-0.05$ to $0.82 ; 4$ studies, 176 participants; $12=38 \%$; low-quality evidence; Analysis 13.5) and medium-term follow-up (SMD $0.35,95 \% \mathrm{Cl} 0.11$ to $0.59 ; 3$ studies, 274 participants; $\left.\right|^{2}=21 \%$; very low-quality evidence; Analysis 13.6).

We were unable to extract data from studies by Brown 2017 and Lee 2017 because Brown 2017 reported mean change in moderate to vigorous physical activity and Lee 2017 reported moderate and vigorous physical activity separately. We emailed authors but received no response. In the study by Brown 2017, both the low dose $(n=14)$ and high dose $(n=12)$ exercise group increased their moderate to vigorous physical activity compared to the usual care group $(n=13)(P$ trend $=<0.001)$ over six months. Lee 2017 reported an increase in moderate to vigorous physical activity in the exercise group ( $\mathrm{n}=62$ ) with no change in the usual care group at three months (group $x$ time interaction, $P<0.01$ ). Data were insufficient to conduct a meta-analysis for change from baseline from more than 12 weeks to six months follow-up and more than six months to 12 months follow-up for objective measures and subjective measures at all time points.

We performed a sensitivity analysis, excluding studies with dietary advice co-interventions up to 12 weeks follow-up after baseline. Objectively measured physical activity suggests no difference in levels of physical activity in the physical activity group compared to usual care in immediate-term follow-up (MD -2.84, 95\% Cl-12.40 to $6.73 ; 3$ studies, 80 participants; $\left.\right|^{2}=0 \%$; Table 1 ). Sensitivity analysis of subjective measures of physical activity suggest an increase in levels of physical activity in the physical activity group compared to the usual care group in immediate-term follow-up (SMD 0.68, 95\% $\mathrm{Cl} 0.33$ to $1.02 ; 3$ studies, 138 participants; $\mathrm{I}^{2}=0 \%$; Table 1 ). At shortterm follow-up, we included studies at low risk of bias and studies that conducted an ITT analysis; this sensitivity analysis suggests no difference between levels of physical activity in the physical activity group compared to the usual care group.

\section{Subgroup analysis}

Subgroup analyses were precluded as we were unable to extract sufficient compatible data to undertake the predefined subgroup analysis

\section{DISCUSSION}

\section{Summary of main results}

\section{Summary of main results}

This review included 16 randomised controlled trials (RCTs) with a total of 992 participants diagnosed with non-advanced colorectal cancer. We are uncertain whether physical activity interventions improve physical function. We found no evidence of effect of physical activity interventions on disease-related mental health, anthropometric measures or levels of physical activity (measured objectively). Interestingy, when levels of physical activity were measured subjectively they were higher in the physical activity

Physical activity interventions for disease-related physical and mental health during and following treatment in people with non- 
group compared to the usual care group at immediate-, short- and medium-term follow-up. We found no evidence of serious adverse events in the intervention or usual care groups in the eight studies that reported on adverse events. There was inconsistency in the measurement and reporting of adverse events between studies. Where reported, adverse events were generally minor. We found evidence of positive effects of physical activity interventions on physical fitness (aerobic fitness, but not other components of fitness), cancer-related fatigue and health-related quality of life (HRQoL) at immediate- and short-term follow-up but not mediumterm follow-up. Only three studies reported medium-term followup for cancer-related fatigue and HRQoL. No studies assessed outcomes at long-term follow-up. One study that assessed fatigue and levels of physical activity at four years is currently awaiting classification (Van Vulpen 2016).

No studies reported on overall survival or recurrence-free survival. Our findings should be interpreted with caution as we rated the quality of evidence between very low to moderate overall. Further, higher quality studies are required to assess the effectiveness and safety of physical activity interventions for non-advanced colorectal cancer patients.

\section{Overall completeness and applicability of evidence}

This systematic review includes studies from nine different countries. All studies except for one were undertaken in higherincome countries (Nuri 2016). This evidence may therefore limit the applicability to low-middle income countries. All studies in this version of the review except for one, exclusively included individuals with non-advanced colorectal cancer; Courneya 2003 included four stage IV participants. The majority of studies included only participants that had finished active treatment, this was apparent across all outcomes, especially at immediateterm follow-up, limiting applicability to those undergoing active treatment. Ethnicity was only reported in three studies; these studies included mostly white people. Level of education was reported in nine studies and there was some variation in the percentage of participants educated to degree level. The physical activity interventions varied somewhat in their frequency, intensity, time, mode and duration, and because of this we are unable to ascertain optimal mode, frequency, intensity or duration of physical activity interventions for effects on the primary and secondary outcomes assessed in this review, limiting recommendations for clinical practice. We were unable to undertake subgroup analysis according to physical activity intervention or participant characteristics, cancer characteristics, treatment stages and length of time since diagnosis due to lack of comparable data, which limits applicability of findings. The objective measure of physical fitness that was used most commonly amongst included studies was the 6-minute walk test. This is not the gold standard of measuring physical fitness and has been shown to underestimate $\mathrm{VO}_{2}$ peak in cancer survivors (Schumacher 2017). This may also limit the applicability of the evidence in this population. The highest incidence of colorectal cancer occurs in people aged between 65 and 74, the mean age of participants included in this review ranged between 51 and 69, this may therefore limit the applicability of this evidence to the population with the highest incidence of colorectal cancer.

For primary outcomes, such as physical function and diseaserelated mental health, the greatest number of studies included in a meta-analysis at any given time point was four. These findings should be interpreted with caution due to the limited number of studies and small sample sizes, especially at longer periods of follow-up, where fewer studies were included. In addition, some of the outcome data included were not normally distributed which may have affected study results. Therefore, findings should be interpreted with caution. We were unable to undertake a metaanalysis of adverse events due to inconsistencies in measurement and reporting of this data. Less than half of the included studies reported on adverse events and four of these reported that no adverse events occurred. In future, RCTs should systematically record and report adverse events and define whether these events are 'related' or 'unrelated' to the intervention. We could not provide an analysis of effects of physical activity interventions on important outcomes, such as overall survival or recurrence-free survival because no included studies reported these outcomes. No studies assessed the long-term effects (more than 12 months followup) of physical activity interventions on any of the primary and secondary outcomes. One study is currently awaiting classification, which reports on four-year effects of exercise on fatigue and levels of physical activity (Van Vulpen 2016). In addition, across all outcomes, few studies conducted follow-up measurements at medium-term follow-up; these findings should be interpreted with caution

\section{Quality of the evidence}

The quality of the findings are reported in Summary of findings 1 for the main comparison. We assessed the quality of evidence for each outcome using GRADE methodology (GRADE Working Group 2004). For the immediate-term follow-up, GRADE revealed low-quality evidence for physical function, adverse events, physical fitness (aerobic fitness) and cancer-related fatigue. Moderate-quality evidence was revealed for HRQoL (immediate-term follow-up) and disease-related mental health (anxiety and depression) at shortterm follow-up. The quality of evidence for change from baseline scores and all other time points, ranged between very low to moderate quality. We downgraded the quality of evidence, namely in light of imprecision and indirectness due to the small number of studies/participants included and considering the majority of studies were conducted in participants who had finished active treatment. In addition, three studies included a health education component which may also introduce indirectness, although only one of these studies reported outcomes at immediate-term followup. Due to the nature of the intervention it was not possible to blind personnel or participants to the intervention, putting all studies at high risk of performance bias, which may be accentuated where participants completed subjective assessments. We found discrepancy in direction of effect of interventions when levels of physical activity were measured subjectively compared to objectively. It has been recently documented that people with cancer self-report their physical activity levels to be nearly four-fold higher when compared to objective physical activity monitoring data (Vassbakk-Brovold 2016). Most of the uncertainty in judging study bias came from a lack of clarity around allocation concealment and blinding of outcome assessors. The 'Risk of bias' table and graph are available in Figure 2 and Figure 3. In addition, some of the data included in the meta-analysis for measures of mental health, cancer-related fatigue, aerobic fitness and levels of physical activity were not normally distributed, which may have affected the quality of the evidence.

Physical activity interventions for disease-related physical and mental health during and following treatment in people with non- 


\section{Potential biases in the review process}

This systematic review included comprehensive search strategies of eight electronic databases, and four clinical trial registries. We (MMG and MAT) independently screened reference lists of all included studies and any relevant systematic reviews identified. We handsearched conference and meeting abstracts of relevant organisations and undertook data extraction and 'Risk of bias judgement' independently. We sent emails to seven authors of studies registered in clinical trial registries with recent completion dates and received correspondence from six of these authors. We emailed four authors of unpublished studies, two of these studies are included in this review (Lewis 2016; McDermott 2017). We sent a further 13 emails to study authors that included colorectal cancer participants alongside other cancer cohorts, to request colorectal cancer participant data separately and emailed authors to request missing data,some did not reply and exclusion of these results may also be a source of bias. We were unable to explore publication bias because there were less than 10 studies included in each comparison. The mean age of participants ranged between 51 and 69 , which is markedly lower than the incidence of colorectal cancer. The review therefore does not include a cohort of the relevant population, which is a source of selection bias. Furthermore, the mean age range of participants may be indicative of participation bias, where healthier participants are more likely to agree to participate in the physical activity intervention research.

A limitation of this review is that three included studies involved additional components, e.g. healthy eating seminars and a dietary information pack (Bourke 2011), health education materials for the usual care group (Courneya 2016), and weekly education sessions on a range of topics (Hubbard 2016), which could have potential synergistic effects of exercise or physical activity on some outcomes. We investigated their effect on outcomes through exclusion in sensitivity analysis when possible.

\section{Agreements and disagreements with other studies or reviews}

To our knowledge only two other systematic reviews have investigated the effectiveness of physical activity interventions in colorectal cancer patients. One if these reviews published in 2014 included 5 RCTs and 238 patients assessing the effect of exercise on HRQoL, fatigue, physical fitness, survival and/ or tumour-associated biomarkers in colorectal cancer patients (Cramer 2014b). The meta-analysis for physical fitness, fatigue and $\mathrm{HRQOL}$ included three studies all of which are included in our review (Bourke 2011; Courneya 2003; Pinto 2013). At shortterm follow-up (corresponding to our immediate-term follow-up) authors observed improvements in physical fitness after aerobic exercise compared with controls which is in agreement with our review. Also in agreement with our review, no studies reported on survival. Conversely, authors found no evidence for short-term effects on quality of life or cancer-related fatigue, whereas we found evidence of favouring effects of physical activity interventions on these outcomes at the same time point. We also observed this effect at short-term follow-up. Cramer 2014b was unable assess outcomes at this time point due to insufficient data. In addition, authors were unable to report on safety data, whereas we reported adverse events narratively due to the extent of variation in measurement and reporting of this outcome. The differences between these reviews are due to the time of the literature search, indeed all of our other included studies were conducted after 2014.
The second review conducted by van Rooijen 2018 highlighted the lack of evidence available on exercise training in those explicitly undergoing active treatment, which was also evident in this review. Six of the seven studies included in the van Rooijen 2018 review included mixed cancer populations, we therefore did not compare results.

\section{AUTHORS' CONCLUSIONS}

\section{Implications for practice}

We are uncertain whether physical activity interventions improve physical function compared to usual care. Physical activity interventions may have no effect on disease-related mental health. Physical activity interventions may be beneficial for aerobic fitness, cancer-related fatigue and HRQOL at immediate-and shortterm follow-up. There were no serious adverse events in any of the studies that provided safety data. Where reported, adverse events were generally minor. The findings of this review should be interpreted with caution due to the low number of studies included and the quality of the evidence. Due to inconsistency in measuring and reporting of this data, more research is required to inform clinical practice. In addition, the current evidence is based on a small number of studies with few participants. The evidence is graded between low and moderate for the main outcomes, which precludes informed decision making in the clinical setting. Adequately powered RCTs of high methodological quality with longer-term follow-up are required to assess the effect of physical activity interventions on disease-related physical and mental health and on survival of people with non-advanced colorectal cancer. Adverse events should also be adequately reported. Further, it would be extremely important to understand whether certain exercise components (mode, frequency, duration and intensity) have optimal effects on physical and disease-related mental health of CRC patients both during and following active treatment.

\section{Implications for research}

This review highlights the need for further large-scale RCTs to assess the effect of physical activity interventions on the diseaserelated physical and mental health of people with non-advanced colorectal cancer. Future RCTs should be of high methodological quality and adequately powered, and include longer-term followup to investigate the sustainability of short-term benefits of physical activity. We identified only two ongoing studies that are investigating disease-free survival, recurrence-free survival and overall survival (Piringer 2017; NCT03885817) and only two studies explicitly stating "side effects of the intervention" and "safety" as outcome measures (Ho 2013; Piringer 2017). It is important for future RCTs to investigate the effects of physical activity on overall survival and recurrence-free survival and report adherence to Enhanced Recovery After Surgery (ERAS) guidelines and length of stay and systematically record and adequately report adverse events, defining whether these events are 'related' or 'unrelated' to the intervention. More research is also required exclusively in those undergoing active cancer treatment for non-advanced colorectal cancer. Indeed, RCTs undertaken with mixed cancer populations should report data separately for the specific cancers, when appropriate. More robust measures to reduce bias, especially in relation to allocation concealment and blinding of outcome assessors are required. In addition, future research should aim to recruit older participants to increase applicability of results to 
those with the highest incidence of colorectal cancer. Importantly, a better understanding of the optimal training duration, pattern, intensity, volume, setting and composition of such interventions will be needed to maximise efficacy. This requires a shift in how we record and report these components.

\section{ACKNOWLEDGEMENTS}

We would like to thank Dr Henning Keinke Andersen and Dr Kristoffer Andersen (Managing Editors), the Cochrane Colorectal Cancer Contact Editor, Dr Nicole Skoetz and Dr Daniel Steffens (peer reviewers) for their help and advice in the development of the Review protocol, Dr Aron Onerup, Dr Rasmus Dahlin Bojesen and Dr Newton Opiyo (peer reviewers) for providing feedback on the review. We would also like to thank Sys Johnsen (Information Specialist) for assisting in the development and adaptation of the search strategy and Dr Lisa A Loughney for her help and advice in the review process. We would like to thank Jill Pearson (consumer referee) and Evelyn (consumer referee) for their assistance and feedback.

MMG has been funded by the Public Health Agency, HSC R \& D Division to undertake this review. MAT and MMC are part funded by the Centre of Excellence for Public Health (Northern Ireland), a UKCRC Public Health Research Centre of Excellence. The UKCRC Public Health Research Centres of Excellence are funded by the British Heart Foundation, Cancer Research UK, Economic and Social Research Council, Medical Research Council, Research and Development Office for the Northern Ireland Health and Social Services and the Wellcome Trust, under the auspices of the UK Clinical Research Collaboration. 


\section{R E F E R E N C E S}

\section{References to studies included in this review}

Bourke 2011 \{published data only\}

Bourke L, Thompson G, Gibson DJ, Daley A, Crank H, Adam I, et al. Pragmatic lifestyle intervention in patients recovering from colon cancer: a randomized controlled pilot study. Archives of Physical Medicine and Rehabilitation 2011;92(5):749-55.

Brown 2017 \{published data only\}

* Brown JC, Damjanov N, Courneya KS, Troxel AB, Zemel BS, Rickels MR, et al. A randomized dose-response trial of aerobic exercise and health-related quality of life in colon cancer survivors. Psycho-Oncology 2018;27(4):1221-8.

Brown JC, Troxel AB, Ky B, Damjanov N, Zemel BS, Rickels MR, et al. A randomized phase II dose-response exercise trial among colon cancer survivors: purpose, study design, methods, and recruitment results. Contemporary Clinical Trials 2016;47:366-75.

Brown JC, Troxel AB, Ky B, Damjanov N, Zemel BS, Rickels MR, et al. Dose-response effects of aerobic exercise among colon cancer survivors: a randomized phase II trial. Clinical Colorectal Cancer 2018;17:32-40.

Brown JC, Zemel BS, Troxel AB, Rickels MR, Damjanov N, $K y B$, et al. Dose-response effects of aerobic exercise on body composition among colon cancer survivors: a randomised controlled trial. British Journal of Cancer 2017;21:1614-20.

\section{Cantarero-Villanueva 2016 \{published data only\}}

Cantarero-Villanueva I, Sánchez-Jiménez A, Galiano-Castillo N, Díaz-Rodríguez L, Martín-Martín L, Arroyo-Morales M.

Effectiveness of lumbopelvic exercise in colon cancer survivors: a randomized controlled clinical trial. Medicine and Science in Sports and Exercise 2016;48(8):1438-46.

\section{Courneya 2003 \{published data only\}}

Courneya KS, Friedenreich CM, Quinney HA, Fields AL, Jones LW, Fairey AS. A randomized trial of exercise and quality of life in colorectal cancer survivors. European Journal of Cancer Care 2003;12(4):347-57.

\section{Courneya 2016 \{published data only\}}

Baylock B, Dhillon HM, Courneya K, O'Callaghan C, O'Brien A, Goddard AM, et al. A phase III study of the impact of a physical activity program on disease-free survival in patients with highrisk stage II or stage III colon cancer: a randomised control trial (NCIC CTG CO.21). Asia-Pacific Journal of Clinical Oncology 2014;10(Suppl 8):199-200.

Baylock B, Dhillon HM, Goddard EM, Turner J, Rice H, Kabourakis $\mathrm{M}$, et al. The colon health and life-long exercise change (challenge) trial: Participant adherence to fitness assessments. Asia-Pacific Journal of Clinical Oncology 2014;10(Suppl 8):200.

* Courneya KS, Booth CM, Gill S, O'Brien P, Vardy J, Friedenreich $\mathrm{CM}$, et al. The colon health and life-long exercise change trial: a randomized trial of the National Cancer
Institute of Canada Clinical Trials Group. Current Oncology 2008;15(6):271-8.

Courneya KS, Vardy J, Gill S, Jonker D, O'Brien P, Friedenreich CM, et al. Update on the colon health and lifelong exercise change trial: a phase III study of the impact of an exercise program on disease-free survival in colon cancer survivors. Current Colorectal Cancer Reports 2014;10(3):321-8.

Courneya KS, Vardy JL, O'Callaghan CJ, Friedenreich CM, Campbell KL, Prapavessis H, et al. Effects of a structured exercise program on physical activity and fitness in colon cancer survivors: one year feasibility results from the CHALLENGE Trial. Cancer Epidemiology, Biomarkers \& Prevention 2016;25(6):969-77.

Vardy J, Dhillon H, Van Der Ploeg H, Goddard E, Clarke S, Spencer L, et al. Challenge: a phase III study of a physical activity program on disease-free survival in patients with high risk stage II/III colon cancer. Asia-Pacific Journal of Clinical Oncology 2010;6:186.

Vardy J, Dhillon HM, van der Ploeg H, Goddard E, Turner J, Kabourakis M, et al. The challenge trial. A phase III study of the impact of a physical activity program on disease-free survival in patients with high-risk stage II or stage III colon cancer: a randomised controlled trial. Asia-Pacific Journal of Clinical Oncology 2013;9:156.

\section{Cramer 2016 \{published data only\}}

* Cramer H, Pokhrel B, Fester C, Meier B, Gass F, Lauche R, et al. A randomized controlled bicenter trial of yoga for patients with colorectal cancer. Psycho-Oncology 2016;25(4):412-20.

Cramer H, Pokhrel B, Gass F, Eisenmann C, Lauche R, Meier B, et al. Hatha yoga for patients with colorectal cancer: a randomized controlled mixed-methods study. Journal of Alternative and Complementary Medicine 2014;20:A52-3.

\section{Hubbard 2016 \{published data only\}}

Hubbard G, Adams R, Campbell A, Kidd L, Leslie S, Munro J, et al. Cardiac rehabilitation to increase physical activity among cancer patients: is it feasible and acceptable? Psycho-Oncology 2016;25:5.

* Hubbard G, Adams R, Campbell A, Kidd L, Leslie SJ, Munro J, et al. Is referral of postsurgical colorectal cancer survivors to cardiac rehabilitation feasible and acceptable? A pragmatic pilot randomised controlled trial with embedded qualitative study. BMJ Open 2016;6:e009284.

Hubbard G, O'Carroll R, Munro J, Mutrie N, Haw S, Mason H, et al. The feasibility and acceptability of trial procedures for a pragmatic randomised controlled trial of a structured physical activity intervention for people diagnosed with colorectal cancer: findings from a pilot trial of cardiac rehabilitation versus usual care (no rehabilitation) with an embedded qualitative study. Pilot \& Feasibility Studies 2016;2:51.

Munro J, Adams R, Campbell A, Campbell S, Donaldson C, Godwin J, et al. CRIB - The use of cardiac rehabilitation services 
to aid the recovery of patients with bowel cancer: a pilot randomised controlled trial (RCT) with embedded feasibility study. BMJ Open 2014;4:e004684.

Watson AJ, Hubbard G, Munro J, Adams R. Is use of cardiac rehabilitation an acceptable and feasible rehabilitation model for patients with colorectal cancer and is a randomised trial of this intervention also acceptable and feasible? Gut 2015;64:A334-5.

\section{Kim 2018 \{published data only\}}

Kim JY, Lee MK, Lee DH, Kang DW, Min JH, Lee JW, et al. Effects of a 12-week home-based exercise program on quality of life, psychological health, and the level of physical activity in colorectal cancer survivors: a randomized controlled trial. Supportive Care in Cancer 2019;27:2933-40.

\section{Lee 2017 \{published data only\}}

* Lee MK, Kim JY, Kim DI, Kang DW, Park JH, Ahn KY, et al. Effect of home-based exercise intervention on fasting insulin and Adipocytokines in colorectal cancer survivors: a randomized controlled trial. Metabolism 2017;76:23-31.

Lee MK, Kim JY, Park JH, Lee J, Kim DI, Kang D, et al. Effect of 12 week home-based exercise program on fasting insulin in stage II-III colorectal cancer survivors: a randomized controlled trial. Journal of Clinical Oncology 2014;32(15 Suppl):3588.

\section{Lewis 2016 \{published data only\}}

Lewis L. A Feasibility Study of an Autonomous Supportive Intervention in People Recovering from CRC: a randomised controlled trial [PhD thesis]. Norwich (UK): University of East Anglia, 2016. [NCT02751892]

\section{McDermott 2017 \{published data only\}}

McDermott L. The Feasibility of a Home-based Walking and Strengthening Intervention on Physiological, Biochemical and Psychological Outcomes in Colorectal Cancer Survivors [PhD thesis]. Belfast (UK): Faculty of Life and Health Sciences of the Ulster University, 2017. [NCT01325909]

\section{Nuri 2016 \{published data only\}}

Nuri R, Moghaddasi M, Darvishi H, Izadpanah A. Effect of aerobic exercise on leptin and ghrelin in patients with colorectal cancer. Journal of Cancer Research \& Therapeutics 2016;12:169-74.

\section{Pinto 2013 \{published data only\}}

Pinto BM, Papandonatos GD, Goldstein MG, Marcus BH, Farrell N, Pinto BM, et al. Home-based physical activity intervention for colorectal cancer survivors. Psycho-Oncology 2013;22:54-64

\section{Van Blarigan 2019 \{published data only\}}

Chan H, Van Loon K, Kenfield SA, Chan JM, Mitchell E, Zhang L, et al. Effect of physical activity trackers and daily text messages on quality-of-life in colorectal survivors (Smart Pace): a pilot randomized controlled trial. www.researchgate.net/ publication/337996933.

NCT02966054. Self-monitoring and reminder texts to increase physical activity after cancer: a pilot randomized controlled trial. clinicaltrials.gov/ct2/show/NCT02966054 (first received 17 November 2017).

* Van Blarigan E, Chan H, Van Loon K, Kenfield SA, Chan JM, Mitchell E, et al. Self-monitoring and reminder text messages to increase physical activity after colorectal cancer (CRC): a pilot randomized controlled trial. BMC Cancer 2019;19(218):1-9.

Van Blarigan E, Van Loon K, Kenfield SA, Chan JM, Mitchell E, $\mathrm{Chan} \mathrm{H}$, et al. Self-monitoring and reminder text messages to increase physical activity after colorectal cancer (CRC): a pilot randomized controlled trial. Journal of Clinical Oncology 2018;36(4 Suppl 615):ascopubs.org/doi/abs/10.1200/ JCO.2018.36.4_suppl.615. [DOI: 10.1200/JCO.2018.36.4suppl.615]

\section{Van Vulpen 2016 \{published data only\}}

May AM, Bosch MJ, Velthuis MJ, Van Der Wall E, Bisschop CN, Los M, et al. Cost-effectiveness analysis of an 18-week exercise programme for patients with breast and colon cancer undergoing adjuvant chemotherapy: The randomised PACT study. BMJ Open 2017;7:e012187.

* Van Vulpen JK, Velthuis MJ, Steins Bisschop CN, Travier N, Van Den Buijs BJ, Backx FJ, et al. Effects of an exercise program in colon cancer patients undergoing chemotherapy. Medicine and Science in Sports and Exercise 2016;48(5):767-75.

Velthuis MJ, May AM, Koppejan-Rensenbrink RA, Gijsen BC, van Breda $E$, de Wit GA, et al. Physical Activity during cancer treatment (PACT) study: design of a randomised clinical trial. BMC Cancer 2010;10:272.

Witlox L, Hiensch AE, Velthuis M, Steins C, Bisschop Maartje Los B, Erdkamp FL, et al. Four-year effects of exercise on fatigue and physical activity in patients with cancer. BMC Medicine 2018;16(1):86.

\section{Waart 2017 \{published data only\}}

* Waart H, Stuiver M, Harten W, Sonke G, Aaronson N. Design of the Physical exercise during adjuvant chemotherapy effectiveness study (PACES): a randomized controlled trial to evaluate effectiveness and cost-effectiveness of physical exercise in improving physical fitness and reducing fatigue. BMC Cancer 2010;10:673.

Waart H, Stuiver M, Sonke G, Harten W, Aaronson N. Intensive physical training during adjuvant treatment. Radiotherapy and Oncology 2014;111(Suppl 1):S213.

Waart H, Stuiver MM, Sonke GS, Van Harten WH, Aaronson NK. Effect of low versus high intensity physical exercise during chemotherapy on physical fitness, fatigue and chemotherapy completion rates: results of a randomized, controlled trial. Psycho-Oncology 2014;23:93.

van Waart H, Stuiver MM, van Harten WH, Geleijn E, de MaakerBerkhof M, Schrama J, et al. Recruitment to and pilot results of the PACES randomized trial of physical exercise during adjuvant chemotherapy for colon cancer. International Journal of Colorectal Disease 2018;33:29-40. 


\section{References to studies excluded from this review}

ACTRN12618001855213 \{published data only\}

ACTRN12618001855213. A peer support program for the longterm maintenance of physical activity and health outcomes in breast, prostate and colorectal cancer survivors [The PEER Study: Peer support for the maintenance of high intensity interval training and health in cancer survivors]. anzctr.org.au/ ACTRN12618001855213.aspx (first received 14 November 2018).

\section{Adamsen 2009 \{published data only\}}

Adamsen L, Quist M, Andersen C, Moller T, Herrstedt J, Kronborg D, et al. Effect of a multimodal high intensity exercise intervention in cancer patients undergoing chemotherapy: randomised controlled trial. BMJ (Online) 2009;339(7726):895-8.

\section{Adlard 2016 \{published data only\}}

Adlard KN, Devin JL, Jenkins DG, Bolam KA, Aitken JF, Chambers SK, et al. The influence of exercise intensity on fatigue in colorectal cancer survivors: a randomized controlled trial. Asia-Pacific Journal of Clinical Oncology 2016;12(Suppl 5):78.

\section{Ahn 2013 \{published data only\}}

Ahn KY, Hur H, Kim DH, Min J, Jeong DH, Chu SH, et al. The effects of inpatient exercise therapy on the length of hospital stay in stages I-III colon cancer patients: randomized controlled trial. International Journal of Colorectal Disease 2013;28(5):643-51.

\section{Allgayer 2004b \{published data only\}}

Allgayer H, Nicolaus S, Schreiber S. Decreased interleukin-1 receptor antagonist response following moderate exercise in patients with colorectal carcinoma after primary treatment. Cancer Detection and Prevention 2004;28(3):208-13.

\section{An 2012 \{published data only\}}

An KY, Lee MK, Kim DI, Kim DH, Min JH, Kim NK, et al. Randomized controlled trial of colorectal cancer exercise program for colorectal cancer patient after surgery. Medicine and Science in Sports and Exercise 2012;44:276-7.

\section{Anderson 2010 \{published data only\}}

Anderson AS, Caswell S, Wells M, Steele RJ, Macaskill S, Anderson AS, et al. "It makes you feel so full of life" LiveWell, a feasibility study of a personalised lifestyle programme for colorectal cancer survivors. Supportive Care in Cancer 2010;18(4):409-15.

\section{Arving 2013 \{published data only\}}

Arving C, Thormodsen I, Brekke G, Mella O, Berntsen S, Nordin K. Early rehabilitation of cancer patients - a randomized controlled intervention study. BMC Cancer 2013;13(9):1-7.

\section{Backman 2014 \{published data only\}}

Backman M, Wengström Y, Johansson B, Sköldengen I, Börjesson S, Tärnbro S, et al. A randomized pilot study with daily walking during adjuvant chemotherapy for patients with breast and colorectal cancer. Acta Oncologica 2014;53(4):510-20.

Malin MB, Yvonne YW, Birgitta BJ, Ida IS, Ake AB. Daily walking during adjuvant chemotherapy for patients with breast and colorectal cancer - a randomized pilot study. European Journal of Cancer 2013;49(Suppl 2):397.

\section{Basen-Engquist 2015 \{published data only\}}

Basen-Engquist K, Parker PA, Eng C, Li YS, Johnson DB, Kee BK, et al. Randomized trial of a home-based exercise intervention for patients with advanced colorectal cancer: effects on physical functioning and activity levels. Journal of Clinical Oncology 2015;33(15 Suppl 9633):ascopubs.org/doi/abs/10.1200/ jco.2015.33.15_suppl.9633.

\section{Beck 2015 \{published data only\}}

Beck AC, Garland EL, Hansen PA, Walker D, Reimers C, Thomas EA, et al. Integrated mindfulness-oriented recovery enhancement (MORE) and physical health intervention for cancer survivors with obesity: preliminary results from a pilot randomized controlled trial. Journal of Clinical Oncology 2015;33(29 Suppl 1):237.

\section{Beeken 2016 \{published data only\}}

Beeken RJ, Croker H, Heinrich M, Smith L, Williams K, Hackshaw A, et al. Study protocol for a randomised controlled trial of brief, habit-based, lifestyle advice for cancer survivors: exploring behavioural outcomes for the Advancing Survivorship Cancer Outcomes Trial (ASCOT). BMJ Open 2016;6(11):e011646.

\section{Bennett 2007 \{published data only\}}

Bennett J, Lyons K, Winters-Stone K, Nail L, Scherer J. Motivational interviewing to increase physical activity in longterm cancer survivors: a randomized controlled trial. Nursing Research 2007;56(1):18-27.

\section{Berntsen 2017 \{published data only\}}

Berntsen S, Aaronson NK, Buffart L, Börjeson S, Demmelmaier I, Hellbom M, et al. Design of a randomized controlled trial of physical training and cancer (Phys-Can) - the impact of exercise intensity on cancer related fatigue, quality of life and disease outcome. BMC Cancer 2017;17:1-12.

Igelstrom H, Berntsen S, Hetlelid K, Asenlof P, Demmelmaier I, Henriksson A, et al. Phys-can feasibility study: preparing for physical training and behavioral medicine strategies. PsychoOncology 2014;23:95.

Nordin K, Johansson B. Physical training and cancer (phys-can)design and description of a multicenter clinical trial within a multidisciplinary consortium. US National Library of Medicine 2014;23(Suppl 3):205.

\section{Broderick 2014 \{published data only\}}

Broderick JM, Guinan E, O'Donnell DM, Hussey J, Tyrrell E, Normand C. Calculating the costs of an 8-week, physiotherapyled exercise intervention in deconditioned cancer survivors in the early survivorship period (the PEACH trial). Physiotherapy 2014;100(2):182-4.

\section{Buffart 2014 \{published data only\}}

Buffart L, Ros W, Chinapaw M, Brug J, Knol D, Korstjens I, et al. Mediators of physical exercise for improvement in cancer survivors' quality of life. Psycho-Oncology 2014;23(3):330-8.

Physical activity interventions for disease-related physical and mental health during and following treatment in people with non- 
Burnham 2002 \{published data only\}

Burnham TR, Wilcox A. Effects of exercise on physiological and psychological variables in cancer survivors. Medicine and Science in Sports and Exercise 2002;34(12):1863-7.

\section{ChiCTROON15005952 \{published data only\}}

ChiCTROON15005952. Exercise Prescription for patients with colorectal cancer. www.chictr.org.cn/historyversionpub.aspx? regno $=$ ChiCTR-OON-15005952 (first registered 3 August 2016).

\section{Demark-Wahnefried 2012 \{published data only\}}

Demark-Wahnefried W, Morey MC, Sloane R, Snyder DC, Miller PE, Hartman TJ, et al. Reach out to enhance wellness home-based diet-exercise intervention promotes reproducible and sustainable long-term improvements in health behaviors, body weight, and physical functioning in older, overweight/ obese cancer survivors. Journal of Clinical Oncology 2012;30(19):2354-61.

Morey MC, Snyder DC, Sloane R, Cohen HJ, Peterson B, Hartman TJ, et al. Effects of home-based diet and exercise on functional outcomes among older, overweight long-term cancer survivors: RENEW: a randomized controlled trial. JAMA 2009;301(18):1883-91.

NCT00303875. Exercise and dietary counseling in improving physical activity, nutrition, and quality of life in older long-term cancer survivors who are overweight. clinicaltrials.gov/ct2/ show/NCT00303875 (first registered 17 March 2006).

Synder DC, Morey MC, Sloane R, Stull V, Cohen HJ, Peterson B, et al. Reach out to ENhancE Wellness in Older Cancer Survivors (RENEW): design, methods and recruitment challenges of a home-based exercise and diet intervention to improve physical function among long-term survivors of breast, prostate, and colorectal cancer. Psycho-Oncology 2009;18(4):429-39.

Winger JG, Mosher CE, Rand KL, Morey MC, Snyder DC, DemarkWahnefried W. Diet and exercise intervention adherence and health-related outcomes among older long-term breast, prostate, and colorectal cancer survivors. Annals of Behavioral Medicine 2014;48(2):235-45.

\section{DeTroye 2018 \{published data only\}}

DeTroye A, Christner M, Eganhouse D, Manning B, Sunkin E, Gregory T. The effects of physical activity on survival in patients with colorectal cancer. Journal of the American Academy of Physician Assistants 2018;31(2):21-5.

\section{Devin 2016 \{published data only\}}

ACTRN12615000908538. The influence of high-intensity compared with moderate-intensity exercise training on the health of colorectal cancer survivors: a randomised controlled trial. www.anzctr.org.au/TrialSearch.aspx (first registered 1 Sep 2015).

Devin JL, Jenkins DG, Sax AT, Hughes GI, Aitken JF, Chambers SK, et al. The influence of exercise intensity and frequency on cardiorespiratory fitness and body composition in colorectal cancer survivors: a randomized controlled trial. AsiaPacific Journal of Clinical Oncology 2016;12(Suppl 5):109.
Devin JL, Sax AT, Hughes GI, Jenkins DG, Aitken JF, Chambers SK, et al. The influence of high-intensity compared with moderate-intensity exercise training on cardiorespiratory fitness and body composition in colorectal cancer survivors: a randomised controlled trial. Journal of Cancer Survivorship 2016;10(3):467-79

\section{Dimeo 2004 \{published data only\}}

Dimeo FC, Thomas F, Raabe-Menssen C, Propper F, Mathias M. Effect of aerobic exercise and relaxation training on fatigue and physical performance of cancer patients after surgery. A randomised controlled trial. Supportive Care in Cancer 2004;12(11):774-9.

\section{Dodd 2010 \{published data only\}}

Dodd MJ, Cho MH, Miaskowski C, Painter PL, Paul SM, Cooper BA, et al. A randomized controlled trial of home-based exercise for cancer-related fatigue in women during and after chemotherapy with or without radiation therapy. Cancer Nursing 2010;33(4):245-57.

\section{DRKS00005793 \{published data only\}}

DRKS00005793. Effects of supervised physical activity on endurance capacity in colorectal cancer patients undergoing ambulatory Chemotherapy. www.drks.de/drks_web/ navigate.do? navigationId=trial.HTML\&TRIAL_ID=DRKS00005793 (first registered 3 Nov 2014).

Eakin 2015 \{published data only\}

Eakin EG, Hayes SC, Haas MR, Reeves MM, Vardy JL, Boyle F, et al. Healthy living after cancer: a dissemination and implementation study evaluating a telephone-delivered healthy lifestyle program for cancer survivors. BMC Cancer 2015;15(992):1-11.

Forbes 2017 \{published data only\}

Forbes CC, Blanchard CM, Mummery WK, Courneya KS. A pilot study on the motivational effects of an internet-delivered physical activity behaviour change programme in Nova Scotian cancer survivors. Psychology and Health 2017;32(2):234-52.

\section{Freitag 2018 \{published data only\}}

Freitag N, Weber PD, Sanders TC, Schulz H, Bloch W, Schumann M. High-intensity interval training and hyperoxia during chemotherapy a case report about the feasibility, safety and physical functioning in a colorectal cancer patient. Medicine 2018;97(24):e11068.

\section{Gabrys 2017 \{published data only\}}

Gabrys L, Sperzel S, Bernhoerster M, Banzer W, Vogt L. Real-time visual activity feedback for physical activity improvement in breast and colon cancer patients. Research in Sports Medicine 2017;25(1):1-10.

\section{Gillis 2014 \{published data only\}}

Gillis C, Li C, Lee L, Awasthi R, Augustin B, Gamsa A, et al. Prehabilitation versus rehabilitation: a randomized control trial in patients undergoing colorectal resection for cancer. Anesthesiology 2014;121(5):937-47. 
Gray 2013 \{published data only\}

Gray NM, Allan JL, Murchie P, Browne S, Hall S, Hubbard G, et al. Developing a community-based intervention to improve quality of life in people with colorectal cancer: a complex intervention development study. BMJ Open 2013;3(4):e002596.

Grimmett 2015 \{published data only\}

Grimmett C, Simon A, Lawson V, Wardle J. Diet and physical activity intervention in colorectal cancer survivors: a feasibility study. European Journal of Oncology Nursing 2015;19(1):1-6.

ISRCTN45454522. Feasibility of a personalised, distancebased lifestyle intervention in colorectal cancer patients. www.isrctn.com/ISRCTN45454522 (first registered 2 Nov 2010).

\section{Hawkes 2014 \{published data only\}}

Gordon LG, Patrao T, Kularatna S, Hawkes AL. A telephonedelivered multiple health behaviour change intervention for colorectal cancer survivors: making the case for cost-effective healthcare. European Journal of Cancer Care 2015;24(6):854-61.

Hawkes A, Pakenham K, Courneya K, Peter B, Chambers S. 'Canchange': A trial of a telephone-delivered lifestyle intervention for colorectal cancer (CRC) survivors. Asia-Pacific Journal of Clinical Oncology 2010;6:193.

Hawkes A, Pakenham K, Courneya KS, Patrao T. A randomised controlled trial of the effects of a telephone-delivered program on health behaviours and quality of life for colorectal cancer survivors ('canchange'). Asia-Pacific Journal of Clinical Oncology 2011;7:80-1.

Hawkes AL, Chambers SK, Pakenham KI, Patrao TA, Baade PD, Lynch BM, et al. Effects of a telephone-delivered multiple health behavior change intervention (CanChange) on health and behavioral outcomes in survivors of colorectal cancer: a randomized controlled trial. Journal of Clinical Oncology 2013;31(18):2313.

Hawkes AL, Gollschewski S, Lynch BM, Chambers S. A telephone-delivered lifestyle intervention for colorectal cancer survivors 'CanChange': a pilot study. Psycho-Oncology 2009;18(4):449-55.

Hawkes AL, Gollschewski S, Lynch BM, Chambers SK. Developing and pilot testing a telephone-delivered lifestyle intervention for colorectal cancer survivors - 'Canchange'. AsiaPacific Journal of Clinical Oncology 2009;5:A215.

Hawkes AL, Pakenham K, Courneya KS, Patrao TA. A randomised trial of the effects of a multiple health behaviour intervention for colorectal cancer survivors on quality of life and psychosocial outcomes ('CanChange'). Asia-Pacific Journal of Clinical Oncology 2012;8:132.

Hawkes AL, Pakenham KI, Chambers SK, Patrao TA, Courneya KS. Effects of a multiple health behavior change intervention for colorectal cancer survivors on psychosocial outcomes and quality of life: a randomized controlled trial. Annals of Behavioral Medicine 2014;48(3):359-70.

Hawkes AL, Pakenham KI, Courneya KS, Gollschewski S, Baade P, Gordon LG, et al. A randomised controlled trial of a tele-based lifestyle intervention for colorectal cancer survivors ('CanChange'): study protocol. BMC Cancer 2009;9:www.researchgate.net/publication/26750592.

Lynch B, Courneya K, Sethi P, Patrao T, Hawkes A. A randomized controlled trial of a multiple health behavior change intervention delivered to colorectal cancer survivors: effects on sedentary behavior. Cancer 2014;120(17):2665-72.

\section{Hernon 2016 \{published data only\}}

Hernon J. Supportive Exercise Programmes for Accelerating Recovery after Major Abdominal Cancer Surgery (PREPARE$A B C)$. Norwich (UK): Norfolk and Norwich University Hospital, 2016.

McCulloch JA, Murdoch J, Varley A, Saxton J, Swart A, Sims E, et al. Process evaluation for the Prepare-ABC study: unpicking the standard pre and post-operative care of colorectal cancer patients. Colorectal Disease 2017;19(Suppl 4):3.

\section{Hung 2016 \{published data only\}}

Hung SL, Lin YH, Yang HY, Kao CC, Tung HY, Wei LH. Pelvic floor muscle exercise for fecal incontinence quality of life after coloanal anastomosis. Journal of Clinical Nursing 2016;25(17-18):2658-68.

\section{Ibfelt 2011 \{published data only\}}

Ibfelt E, Rottmann N, Kjaer T, Høybye MT, Ross L, Frederiksen K, et al. No change in health behavior, bmi or self-rated health after a psychosocial cancer rehabilitation: results of a randomized trial. Acta Oncologica 2011;50(2):289-98.

ISRCTN56928944 \{published data only\}

ISRCTN56928944. The effect of postsurgery exercise on recovery from colorectal cancer. isrctn.com/ISRCTN56928944 (first registered 17 Feb 2012).

\section{ISRCTN62859294 \{published data only\}}

ISRCTN62859294. The feasibility of performing a walking programme in patients with rectal cancer undergoing chemoradiotherapy (the REx trial). isrctn.com/ISRCTN62859294 (first registered 21 Jan 2014).

\section{ISRCTN96374224 \{published data only\}}

ISRCTN96374224. CLASP Renewed Online Feasibility Study. isrctn.com/ISRCTN96374224 (first registered 31 July 2017).

\section{Kalter 2015 \{published data only\}}

Kalter J, Buffart L, Korstjens I, Weert E, Brug J, Verdonck-de LI, et al. Moderators of the effects of group-based physical exercise on cancer survivors' quality of life. Supportive Care in Cancer 2015;23(9):2623-31.

\section{Kampshoff 2015 \{published data only\}}

Kampshoff C, Chinapaw M, Brug J, Twisk J, Schep G, Nijziel M, et al. Randomized controlled trial of the effects of high intensity and low-to-moderate intensity exercise on physical fitness and fatigue in cancer survivors: results of the Resistance and Endurance exercise After ChemoTherapy (REACT) study. BMC Medicine 2015;13(1):1-12. 
Kanera 2017 \{published data only\}

Kanera IM, Bolman CA, Willems RA, Mesters I, Lechner L. Lifestyle-related effects of the web-based Kanker Nazorg Wijzer (Cancer Aftercare Guide) intervention for cancer survivors: a randomized controlled trial. Journal of Cancer Survivorship 2016;10(5):883-97.

Kanera IM, Willems RA, Bolman CA, Mesters I, Verboon P, Lechner L. Long-term effects of a web-based cancer aftercare intervention on moderate physical activity and vegetable consumption among early cancer survivors: a randomized controlled trial. International Journal of Behavioral Nutrition and Physical Activity 2017;14(1):19.

\section{laStayo 2011 \{published data only\}}

NCT00335491. Exercise in improving mobility and reducing fatigue and/or weakness in older cancer survivors. clinicaltrials.gov/ct2/show/NCT00335491 (first registered 12 June 2006).

laStayo PC, Marcus RL, Dibble LE, Smith SB, Beck SL. Eccentric exercise versus usual-care with older cancer survivors: the impact on muscle and mobility - an exploratory pilot study. BMC Geriatrics 2011;11(5):1-10.

\section{Lee 2013 \{published data only\}}

Lee DH, Kim JY, Lee MK, Lee C, Min J-H, Jeong DH, et al. Effects of a 12-week home-based exercise program on the level of physical activity, insulin, and cytokines in colorectal cancer survivors: a pilot study. Supportive Care in Cancer 2013;21(9):2537-45.

\section{Lee 2018a \{published data only\}}

Lee CF, Ho J WC, Fong DYT, Macfarlane J, Cerin E, Lee AM, et al. Dietary and physical activity interventions for colorectal cancer survivors: a randomized controlled trial. Scientific Reports 2018;8(5731):1-9.

\section{Ligibel 2012 \{published data only\}}

Ligibel J, Meyerhardt J, Najita J, Shockro L, Campbell N, Pierce J. Abstract PD08-09: impact of a Telephone-Based Exercise Intervention on Physical Activity Behaviors and Fitness in a Cooperative Group Setting. Cancer Research 2010;70(Suppl 24):PD 08-09.

Ligibel JA, Meyerhardt J, Pierce JP, Najita J, Shockro L, Campbell N, et al. Impact of a telephone-based physical activity intervention upon exercise behaviors and fitness in cancer survivors enrolled in a cooperative group setting. Breast Cancer Research and Treatment 2012;132(1):205-13.

NCT00548236. The Active After Cancer Trial (AACT). clinicaltrials.gov/ct2/show/NCT00548236 (first registered 21 October 2007)

\section{Lin 2011 \{published data only\}}

Lin KY, Tsauo JY. Effects of supervised exercise intervention in patients with colorectal cancer undergoing chemotherapy. Physiotherapy 2011;97:eS693.

\section{Lin 2014 \{published data only\}}

Lin KY, Shun SC, Lai YH, Liang JT, Tsauo JY. Comparison of the effects of a supervised exercise program and usual care in patients with colorectal cancer undergoing chemotherapy. Cancer Nursing 2014;37(2):E21-9.

\section{Lin 2016 \{published data only\}}

Lin YH, Yang HY, Hung SL, Chen HP, Liu KW, Chen TB, et al. Effects of pelvic floor muscle exercise on faecal incontinence in rectal cancer patients after stoma closure. European Journal of Cancer Care 2016;25(3):449-57.

\section{Loughney 2014 \{published data only\}}

Loughney L, West M, Kemp GW, Grocott MP, Jack S. Exercise regimen post chemoradiotherapy in patients with operable rectal cancer (empower). Medicine and Science in Sports and Exercise 2014;46(5S):365-6.

\section{Lyons 2017 \{published data only\}}

Lyons KD, Newman R, Adachi-Mejia AM, Whipple J, Hegel MT. Content analysis of a participant-directed intervention to optimize activity engagement of older adult cancer survivors. OTJR: Occupation, Participation, and Health 2017;38(1):38-45.

\section{Macleod 2018 \{published data only\}}

Macleod M, Steele RJ, O'Carroll RE, Wells M, Campbell A Sugden JA, et al. Feasibility study to assess the delivery of a lifestyle intervention (TreatWELL) for patients with colorectal cancer undergoing potentially curative treatment. BMJ Open 2018;8(6):e021117.

\section{Mayer 2017 \{published data only\}}

Mayer DK, Landucci G, Awoyinka L, Atwood AK, Carmack CL, Demark-Wahnefried W, et al. SurvivorCHESS to increase physical activity in colon cancer survivors: can we get them moving? Journal of Cancer Survivorship 2017;12(1):82-94.

\section{McCall 2015 \{published data only\}}

McCall M, McDonald M, Thorne S, Ward A, Heneghan C. Yoga for health-related quality of life in adult cancer: a randomized controlled feasibility study. Evidence-based Complementary and Alternative Medicine 2015;2015(816820):1-12

\section{Meyerhardt 2017 \{published data only\}}

Meyerhardt JA, Irwin ML, Jones L, Zhang S, Campbell N, Brown JC, et al. Multicenter, randomized phase II trial of physical activity (PA), metformin (Met), or the combination on metabolic biomarkers in stage I-III colorectal (CRC) and breast cancer (BC) survivors. Journal of Clinical Oncology 2017;35(Suppl 15):10059.

\section{Midtgaard 2011 \{published data only\}}

Midtgaard J, Stage M, Møøller T, Andersen C, Quist M, Røørth M, et al. Exercise may reduce depression but not anxiety in selfreferred cancer patients undergoing chemotherapy. Post-hoc analysis of data from the "Body \& Cancer" trial. Acta Oncologica 2011;50(5):660-9.

\section{Min 2017 \{published data only\}}

Min J-h, Ki-yong A, Hyuna P, Wonhee C, Hye Jeong J, Nam Kyu K, et al. The effect of post-operative exercise in colorectal cancer 
patients: a pilot randomized controlled trial (RCT) study. Asian Oncology Nursing 2017;17(1):29-36.

\section{Morielli 2018 \{published data only\}}

Morielli AR, Usmani N, Boule NG, Severin D, Tankel K, Nijjar T, et al. Exercise during and after neoadjuvant rectal cancer treatment (the EXERT trial): study protocol for a randomized controlled trial. Trials 2018;19(1):35.

\section{NCT00373022 \{published data only\}}

NCT00373022. Moderate physical activity in helping patients recover physically and emotionally from stage II or stage III colorectal cancer. clinicaltrials.gov/ct2/show/NCT00373022 (first registered 7 September 2006).

\section{NCT00977613 \{published data only\}}

NCT00977613. Adherence to a recommended exercise regimen in colorectal cancer patients. clinicaltrials.gov/ct2/show/ NCT00977613 (first received 16 September 2009).

NCT00985400 \{published data only\}

NCT00985400. Doctor-recommended home-based exercise program or relaxation training in improving physical function and controlling symptoms in patients with stage iv or recurrent colon cancer that cannot be removed by surgery. clinicaltrials.gov/ct2/show/NCT00985400 (first registered 28 September 2009).

\section{NCT01032590 \{published data only\}}

NCT01032590. Internet-based weight-loss program for colorectal cancer survivors. clinicaltrials.gov/ct2/show/ NCT01032590 (first registered 15 Dec 2009).

\section{NCT01146769 \{published data only\}}

NCT01146769. Early pelvic floor muscle training improves pelvic floor muscle strength in patient after low anterior resection. clinicaltrials.gov/ct2/show/NCT01146769 (first registered 22 June 2010).

\section{NCT01210313 \{published data only\}}

NCT01210313. Physical activity for reduction of recurrence rate after adjuvant chemotherapy for localised colorectal carcinoma. clinicaltrials.gov/ct2/show/NCT0121031 (first registered 28 September 2010).

\section{NCT01325909 \{published data only\}}

NCT01325909. Exercise training in colorectal cancer patients. clinicaltrials.gov/ct2/show/NCT01325909 (first registered 30 March 2011).

\section{NCT01453452 \{published data only\}}

NCT01453452. S1008: Exercise, diet, \& counseling in improving weight loss in overweight female breast or colorectal cancer survivors. clinicaltrials.gov/ct2/show/NCT01453452 (first registered 17 October 2011).

\section{NCT01991847 \{published data only\}}

NCT01991847. Tertiary prevention by exercise in colorectal cancer therapy. clinicaltrials.gov/ct2/show/NCT01991847 (first registered 25 Nov 2013).
NCT02052050 \{published data only\}

NCT02052050. Core stability program in colorectal cancer survivors. clinicaltrials.gov/ct2/show/NCT02052050 (first registered 31 Jan 2014).

\section{NCT02056691 \{published data only\}}

NCT02056691. EDICT - exercise induced changes in colorectal cancer tissues. clinicaltrials.gov/ct2/show/NCT02056691 (first registered 6 Feb 2014).

NCT02264496 \{published data only\}

NCT02264496. Prospective randomised trial of exercise and or antioxidants in colorectal cancer patients undergoing surgery. clinicaltrials.gov/ct2/show/NCT02264496 (first received 15 October 2014).

NCT02442583 \{published data only\}

NCT02442583. Reducing Sedentary Behaviors Among Colorectal Cancer Survivors. clinicaltrials.gov/ct2/show/NCT02442583 (first registered 13 May 2015).

\section{NCT02512263 \{published data only\}}

NCT02512263. Assessment of Adherence and Efficiency of a Home-based Training Program on Muscular Strength, Endurance and Qol for Colon Cancer Patients. ichgcp.net/ clinical-trials-registry/NCT02512263 (first registered 7 Jan 2015).

\section{NCT02522520 \{published data only\}}

NCT02522520. Pedometer intervention and health effects for sedentary colorectal cancer patients during adjuvant chemotherapy. clinicaltrials.gov/ct2/show/NCT02522520 (first registered 13 August 2015).

\section{NCT02586701 \{published data only\}}

NCT02586701. Supervised versus non-supervised exercise on adherence and functional outcomes in colorectal patients. ichgcp.net/clinical-trials-registry/NCT02586701 (first registered 22 Oct 2015).

\section{NCT02647398 \{published data only\}}

NCT02647398. Study comparing two strategies of exercise in breast and colon cancer survivors and their impact on fatigue. clinicaltrials.gov/ct2/show/NCT02647398 (first registered 6 Jan 2016).

\section{NCT02677389 \{published data only\}}

NCT02677389. Survivorship care plan in promoting physical activity in breast or colorectal cancer survivors in Wisconsin. clinicaltrials.gov/ct2/show/NCT02677389 (first registered 9 Feb 2016).

NCT02837159 \{published data only\}

NCT02837159. A Mobile Aplication for the Promotion of Healthy Lifestyle Habits in Patients With Colorectal Cancer. clinicaltrials.gov/ct2/show/NCT02837159 (first registered 19 July 2016).

NCT02889276 \{published data only\}

NCT02889276. Effects of functional exercise on fitness and QoL in cancer survivors. clinicaltrials.gov/ct2/show/NCT02889276 (first registered 5 September 2016). 
NCT03036436 \{published data only\}

NCT03036436. The IMPETUS cancer trial. A technology delivered physical activity intervention in cancer. clinicaltrials.gov/ct2/ show/NCT03036436 (first registered 30 Jan 2017).

\section{NCT03082495 \{published data only\}}

NCT03082495. Exercise during and after neoadjuvant rectal cancer treatment. clinicaltrials.gov/ct2/show/NCT03082495 (first registered 17 March 2017).

\section{NCT03120104 \{published data only\}}

NCT03120104. Physical exercise for colorectal cancer patients after transanal total mesorectal excision. clinicaltrials.gov/ct2/ show/NCT03120104 (first registered 19 April 2017).

\section{NCT03232814 \{published data only\}}

NCT03232814. Walk on: a community-based approach to increase physical activity among men treated for colorectal cancer. clinicaltrials.gov/ct2/show/NCT03232814 (first registered 28 July 2017).

\section{NCT03630354 \{published data only\}}

NCT03630354. Exercising together for couples coping with cancer. clinicaltrials.gov/ct2/show/NCT03630354 (first registered 14 August 2018).

\section{NTR6383 \{published data only\}}

NTR6383. The effect of pelvic floor muscle training on bowel symptoms after low anterior resection for rectal cancer. www.trialregister.nl/trial/6227 (first registered 23 Jan 2017).

\section{Oh 2008 \{published data only\}}

Oh B, Butow P, Mullan B, Clarke S. Medical qigong for cancer patients: Pilot study of impact on quality of life, side effects of treatment and inflammation. American Journal of Chinese Medicine 2008;36(3):459-72.

\section{Onerup 2017 \{published data only\}}

Onerup A, Angenete E, Bock D, Börjesson M, Olsén MF, Gillheimer EG, et al. The effect of pre- and post-operative physical activity on recovery after colorectal cancer surgery (PHYSSURG-C): study protocol for a randomised controlled trial. Trials 2017;18:1-10.

Onerup A, Thorn SE, Angenete E, Bock D, Gryback-Gillheimer E, Haglind $E$, et al. Physical activity before and after colorectal surgery and its effect on IGF-1, IGFBP-3 and HbA1c: The PHYSSURG-C randomized controlled trial. Colorectal Disease 2017;19(Suppl 2):108.

Onerup A, Thorn SE, Angenete E, Bock D, Gryback-Gillheimer E, Haglind $E$, et al. The effect of pre-and postoperative physical activity on recovery after colorectal cancer surgery (PHYSSURG$\mathrm{C})$ : A randomized controlled trial. Colorectal Disease 2017;19(Suppl 2):141.

\section{Phipps 2018 \{published data only\}}

Phipps AI, Shi Q, Zemla TJ, Dotan E, Gill S, Goldberg RM, et al. Physical activity and outcomes in patients with stage III colon cancer: a correlative analysis of phase III trial NCCTG N0147
(Alliance). Cancer Epidemiology Biomarkers and Prevention 2018;27(6):696-703.

Piringer 2013 \{published data only\}

Piringer G, Fridrik M, Fridrik A, Zabernigg A, Greil R, Eisterer W, et al. Endurance exercise to reduce the recurrence rate after adjuvant chemotherapy for colorectal cancer. Onkologie 2013;36:69-70.

Piringer 2018 \{published data only\}

Piringer G, Fridrik M, Fridrik A, Leiherer A, Zabernigg A, Greil R, et al. A prospective, multicenter pilot study to investigate the feasibility and safety of a 1-year controlled exercise training after adjuvant chemotherapy in colorectal cancer patients. Supportive Care in Cancer 2018;26(4):1345-52.

Ratjen 2018 \{published data only\}

Ratjen I, Schafmayer C, Enderle J, di Giuseppe R, Waniek S, Koch $\mathrm{M}$, et al. Health-related quality of life in long-term survivors of colorectal cancer and its association with all-cause mortality: a German cohort study. BMC Cancer 2018;18(1156):1-15.

\section{Ray 2018 \{published data only\}}

Ray AD, Twarozek AM, Williams BT, Erwin DO, Underwood W, Mahoney MC. Exercise in African American and white colorectal cancer survivors: a mixed-methods approach. Rehabilitation Oncology 2018;36(4):188-97.

\section{Sandler 2017 \{published data only\}}

Sandler C, Goldstein D, Horsfield S, Bennett BK, Friedlander M, Bastick PA, et al. TOPS: a randomised controlled trial of a multidisciplinary intervention for post-cancer fatigue. Journal of Clinical Oncology 2015;33(15 Suppl 1):9571.

Sandler CX, Goldstein D, Horsfield S, Bennett BK, Friedlander M, Bastick PA, et al. Randomized evaluation of cognitivebehavioral therapy and graded exercise therapy for postcancer fatigue. Journal of Pain and Symptom Management 2017;54(1):74-84

Sellar 2014 \{published data only\}

Sellar CM, Bell GJ, Haennel RG, Au HJ, Chua N, Courneya KS. Feasibility and efficacy of a 12-week supervised exercise intervention for colorectal cancer survivors. Applied Physiology Nutrition and Metabolism 2014;39(6):715-23.

\section{Sohl 2016 \{published data only\}}

Sohl S, Danhauer S, Birdee G, Nicklas B, Yacoub G, Aklilu M, et al. A brief yoga intervention implemented during chemotherapy for colorectal cancer: a randomized controlled pilot study. Psycho-Oncology 2015;24:253.

Sohl SJ, Danhauer SC, Birdee GS, Nicklas BJ, Yacoub G, Aklilu M, et al. A brief yoga intervention implemented during chemotherapy: a randomized controlled pilot study. Complementary Therapies in Medicine 2016;25:139-42.

Sprod 2015 \{published data only\}

Sprod LK, Fernandez ID, Janelsins MC, Peppone LJ, Atkins JN, Giguere J, et al. Effects of yoga on cancer-related fatigue and 
global side-effect burden in older cancer survivors. Journal of Geriatric Oncology 2015;6(1):8-14.

\section{Ungar 2016 \{published data only\}}

Ungar N, Sieverding M, Weidner G, Ulrich CM, Wiskemann J. A self-regulation-based intervention to increase physical activity in cancer patients. Psychology, Health and Medicine 2016;21(2):163-75.

\section{van Rooijen 2018 \{published data only\}}

van Rooijen SJ, Engelen MA, Scheede-Bergdahl C, Carli F, Roumen RM, Slooter GD, et al. Systematic review of exercise training in colorectal cancer patients during treatment. Scandinavian Journal of Medicine and Science in Sports 2018;28(2):360-70.

\section{Wang 2012 \{published data only\}}

Wang Q, Suo J, Jiang J, Wang C, Zhao YQ, Cao X. Effectiveness of fast-track rehabilitation versus conventional care in laparoscopic colorectal resection for elderly patients: a randomized trial. Colorectal Disease 2012;14(8):1009-13.

\section{West 2015 \{published data only\}}

West MA, Loughney L, Jack S, Grocott MP, Kemp GJ. Exercise training following neoadjuvant chemoradiotherapy in rectal cancer improves mitochondrial function - a randomized controlled trial. British Journal of Surgery 2015;102:2-2.

\section{Zopf 2018 \{published data only\}}

Zopf EM, Schulz H, Poeschko J, Schroder K, Bloch W, Baumann FT. Effects of supervised aerobic exercise on quality of life and fatigue in colorectal cancer patients undergoing adjuvant chemotherapy. Asia-Pacific Journal of Clinical Oncology 2018;14(Suppl 7):143.

\section{References to studies awaiting assessment}

De Backer 2007 \{published data only\}

De Backer IC, Van Breda E, Vreugdenhil A, Nijziel MR, Kester AD, Schep $\mathrm{G}$. High-intensity strength training improves quality of life in cancer survivors. Acta Oncologica 2007;46(8):1143-51.

\section{Golsteijn 2017 \{published data only\}}

Golsteijn RH, Bolman C, Peels DA, De Vries H, Lechner L. Motivating (former) cancer patients to increase their physical activity: the computer tailored oncoactive + project. PsychoOncology 2013;22(10):159.

Golsteijn RH, Bolman C, Peels DA, Volders E, Vries H, Lechner L, et al. A web-based and print-based computer-tailored physical activity intervention for prostate and colorectal cancer survivors: a comparison of user characteristics and intervention use. Journal of Medical Internet Research 2017;19(8):1-1.

Golsteijn RH, Bolman C, Volders E, Peels D, Vries H, Lechner L. Development of an e-health physical activity intervention for prostate and colorectal cancer survivors: the oncoactive intervention. Psycho-Oncology 2014;23:258.

Golsteijn RH, Bolman C, Volders E, Peels DA, de Vries H, Lechner L. Development of a computer-tailored physical activity intervention for prostate and colorectal cancer patients and survivors: OncoActive. BMC Cancer 2017;17(446):1-19.

\section{Ho 2013 \{published data only\}}

Ho JWC, Lee AM, Macfarlane DJ, Fong DY, Leung S, Cerin E, et al. Study protocol for "Moving Bright, Eating Smart"- A phase 2 clinical trial on the acceptability and feasibility of a diet and physical activity intervention to prevent recurrence in colorectal cancer survivors. BMC Public Health 2013;13(487):1-10.

NCT01708824. Diet and Physical Activity Intervention in CRC Survivors. clinicaltrials.gov/ct2/show/NCT01708824 (first registered 17 October 2012).

\section{Houborg 2006 \{published data only\}}

Houborg K, Jensen M, Rasmussen P, Gandrup P, Schroll M, Laurberg S. Postoperative physical training following colorectal surgery: a randomised, placebo-controlled study. Scandinavian Journal of Surgery 2006;95(1):17-22.

Houborg KB, Jensen MB, Hessov I, Laurberg S. Little effect of physical training on body composition and nutritional intake following colorectal surgery-a randomised placebo-controlled trial. European Journal Clinical Nutrition 2005;59(8):969-77.

\section{ISRCTN07465566 \{published data only\}}

ISRCTN07465566. A pilot study to test the effects of physical activity consultations on the physical activity levels and other health outcomes of people living with colorectal cancer and their partners. www.isrctn.com/ISRCTN07465566 (first registered 13 Dec 2011).

\section{Jacobsen 2013 \{published data only\}}

Jacobsen PB, Phillips KM, Jim HS, Small BJ, Faul LA, Meade CD, et al. Effects of self-directed stress management training and home-based exercise on quality of life in cancer patients receiving chemotherapy: a randomized controlled trial. PsychoOncology 2013;22(6):1229-35.

\section{Lee 2018 \{published data only\}}

Lee MK, Kim NK, Jeon JY. Effect of the 6-week home-based exercise program on physical activity level and physical fitness in colorectal cancer survivors: a randomized controlled pilot study. PloS One 2018;13(4):e0196220.

\section{Maxwell-Smith 2019 \{published data only\}}

Maxwell-Smith C, Hince D, Cohen PA, Bulsara MK, Boyle T, Platell C, et al. A randomized controlled trial of WATAAP to promote physical activity in colorectal and endometrial cancer survivors. Psycho-Oncology 2019;28(7):1420-9.

\section{Moller 2015 \{published data only\}}

Moller T, Lillelund C, Andersen C, Bloomquist K, Bang Christensen $\mathrm{K}$, Ejlertsen $\mathrm{B}$, et al. Use of exercise in patients with breast cancer. Supportive Care in Cancer 2015;1 (Meeting Abstracts).

Moller T, Lillelund C, Andersen C, Ejlertsen B, Norgaard L, Christensen KB, et al. At cancer diagnosis: a 'window of opportunity' for behavioural change towards physical activity. A randomised feasibility study in patients with colon and breast cancer. BMJ Open 2013;3(11):e003556. 


\section{NCT02403024 \{published data only\}}

NCT02403024. Feasibility and efficacy of interval walking in patients with colorectal cancer. clinicaltrials.gov/ct2/show/ NCT02403024 (first registered 31 March 2015).

\section{NCT02564835 \{published data only\}}

NCT02564835. Effects of yoga on cognitive and immune function in colorectal cancer. clinicaltrials.gov/ct2/show/ NCT02564835 (first registered 1 Oct 2015).

\section{Park 2015 \{published data only\}}

Park JH, Lee J, Oh M, Park H, Chae J, Kim DI, et al. The effect of oncologists' exercise recommendations on the level of exercise and quality of life in survivors of breast and colorectal cancer: a randomized controlled trial. Cancer 2015;121(16):2740-48.

\section{Schulz 2002 \{published data only\}}

Schulz T, Lotzerich H, Niemeier B, Peters C, Michna H. Effects of moderate exercise training on immunological and psychological parameters in the rehabilitation of colon cancer patients. Journal of Cancer Research and Clinical Oncology 2002;128(Suppl 1):158.

\section{Schwartz 2009 \{published data only\}}

Schwartz AL, Winters-Stone K. Effects of a 12-month randomized controlled trial of aerobic or resistance exercise during and following cancer treatment in women. Physician and Sports Medicine 2009;37(3):62-7.

\section{Shun 2016 \{published data only\}}

Shun SC, Lin BR, Jin-Tung Liang JT, Yun-Jen Chou YJ. Effects of individual exercise education on decreasing fatigue and psychological distress in patients in with colorectal cancer after surgery: a clinical randomized control trial. Supportive Care in Cancer 2016;24(1 Suppl 1):S78.

\section{References to ongoing studies}

\section{ChiCTRIOR17012037 \{published data only\}}

ChiCTRIOR17012037. Effects of resistance training and aerobic exercise on functional recovery and quality of life of patients with colorectal cancer after surgery. www.chictr.org.cn/ showproj.aspx?proj=20476 (first received 18 July 2017).

\section{NCT02191969 \{published data only\}}

NCT02191969. Physical activity intervention for older patients during chemotherapy for colorectal cancer. clinicaltrials.gov/ ct2/show/NCT02191969 (first received 16 July 2014).

\section{NCT02538913 \{published data only\}}

NCT02538913. Exercise training for rectal cancer patients. A randomized controlled trial. clinicaltrials.gov/ct2/show/ NCT02538913 (first received 2 September 2015).

\section{NCT03064308 \{published data only\}}

NCT03064308. The assessment of the feasibility of a home based exercise programme in the older patient following major surgery. clinicaltrials.gov/ct2/show/NCT03064308 (first received 27 Feb 2017).
NCT03210129 \{published data only\}

NCT03210129. Motivational interviewing to increase physical activity behaviour in cancer patients. clinicaltrials.gov/ct2/ show/NCT03210129 (first received 6 July 2017).

\section{NCT03212079 \{published data only\}}

NCT03212079. Physical activities by technology help (PATH). clinicaltrials.gov/ct2/show/NCT03212079 (first received 11 July 2017).

NCT03252821 \{published data only\}

NCT03252821. Effects of high-intensity training compared to resistance training in cancer patients undergoing radiotherapy. clinicaltrials.gov/ct2/show/NCT03252821 (first received 17 August 2017).

NCT03885817 \{published data only\}

NCT03885817. Physically active during cancer treatment (FAKT). clinicaltrials.gov/ct2/show/NCT03885817 (first received 22 March 2019).

\section{Piringer 2017 \{published data only\}}

Annual meeting of the Austrian society of haematology and medical oncology. Phase III randomized trial of endurance exercise following adjuvant chemotherapy for colorectal cancer "aBCSG C08-II trial". Annual Meeting of the Austrian Society of Haematology and Medical Oncology; 2017 Memo - Magazine of European Medical Oncology; Austria 2017;10(2 Suppl 1):45.

\section{Additional references}

\section{ACSM 2009}

American College of Sports Medicine (ACSM). ACSM's Guidelines for Graded Exercise Testing and Prescription. 8th edition. Philadelphia: Lippincott Williams \& Wilkins, 2009.

\section{ACSM 2014}

American College of Sports Medicine (ACSM). ACSM's Guidelines for Graded Exercise Testing and Prescription. 9th edition. Philadelphia: Lippincott Williams \& Wilkins, 2014.

\section{Al-Majid 2009}

Al-Majid S, Gray P. A biobehavioral model for the study of exercise interventions in cancer-related fatigue. Biological Research for Nursing 2009;10(4):381-91. [DOI: 10.1177/1099800408324431]

\section{Allemani 2015}

Allemani C, Weir HK, Carreira H, Harewood R, Spika D, Wang X. Global surveillance of cancer survival 19952009: analysis of individual data for 25676887 patients from 279 population based registries in 67 countries (CONCORD-2). Lancet 2015;385(9972):977-1010. [DOI: 10.1016/ S0140-6736(14)62038-9]

\section{Allgayer 2004a}

Allgayer H, Nicolaus S, Schreiber S. Decreased interleukin-1 receptor antagonist response following moderate exercise in patients with colorectal carcinoma after primary treatment. 
Cancer Detection and Prevention 2004;28:208-13. [DOI: 10.1016/ j.cdp.2004.02.001]

\section{Allgayer 2008}

Allgayer H, Owen RW, Nair J, Spiegelhalder B, Streit J, Reichel C, et al. Short-term moderate exercise programs reduce oxidative DNA damage as determined by high-performance liquid chromatography-electrospray ionization-mass spectrometry in patients with colorectal carcinoma following primary treatment. Scandinavian Journal of Gastroenterology 2008;43(8):971-8. [DOI: dx.doi.org/10.1080/00365520701766111]

\section{Arnold 2017}

Arnold M, Sierra MS, Laversanne M, Soerjomateram I, Jemal A, Bray F. Global patterns and trends in colorectal cancer incidence and mortality. Gut 2017;66:683-91. [DOI: 10.1136/ gutjnl-2015-310912]

\section{Baker 2005}

Baker F, Denniston M, Smith T, West MM. Adult cancer survivors: how are they faring? Cancer 2005;104(S11):2565-76. [DOI: $10.1002 /$ cncr.21488]

\section{BASES 2011}

British Association of Sport and Exercise Science (BASES). The BASES expert statement on exercise and cancer survivorship. www.bases.org.uk/BASES-Expert-statements (accessed 15 June 2017).

\section{Birgisson 2005}

Birgisson H, Påhlman L, Gunnarsson U, Glimelius B. Occurrence of second cancers in patients treated with radiotherapy for rectal cancer. Journal of Clinical Oncology 2005;23(25):6126-31. [DOI: 10.1200/JCO.2005.02.543]

\section{Bower 2014}

Bower JE. Cancer-related fatigue: mechanisms, risk factors, and treatments. Nature Reviews Clinical Oncology 2014;11(10):597-609. [DOI: 10.1038/nrclinonc.2014.127]

\section{Caspersen 1985}

Caspersen CJ, Powell KE, Christenson GM. Physical activity, exercise and physical fitness: definitions and distinctions for health related research. Public Health Reports 1985;100(2):126-31. [DOI: 10.2307/20056429]

\section{Christensen 1982}

Christensen T, Bendix T, Kehlet $\mathrm{H}$. Fatigue and cardiorespiratory function following abdominal surgery. British Journal of Surgery 1982;69(7):417-9. [DOI: 10.1002/bjs.1800690721]

\section{Coleman 2011}

Coleman MP, Forman D, Bryant H, Butler J, Rachet B, Maringe C, et al. Cancer survival in Australia, Canada, Denmark, Norway, Sweden and the UK, 1995-2007 (the International Cancer Benchmarking Partnership): an analysis of population-based cancer registry data. Lancet 2011;377:127-38.

\section{Courneya 2007}

Courneya KS, Freidenreich CM. Physical activity and cancer control. Seminars in Nursing Oncology 2007;23(4):242-52. [DOI: 10.1016/j.soncn.2007.08.002]

\section{Covidence 2018 [Computer program]}

Veritas Health Innovation Covidence. Version accessed 16 January 2018. Melbourne, Australia: Veritas Health Innovation, 2018.Available at covidence.org.

\section{Cramer 2014a}

Cramer L, Hildebrandt B, Kung T, Wichmann K, Springer J, Doehner W, et al. Cardiovascular function and predictors of exercise capacity in patients with colorectal cancer. Journal of the American College of Cardiology 2014;64(13):1310-19. [DOI: http://dx.doi.org/10.1016/j.jacc.2014.07.948]

\section{Cramer 2014b}

Cramer H, Lauche R, Klose P, Dobos G, Langhorst J. A systematic review and meta-analysis of exercise interventions for colorectal cancer patients. European Journal of Cancer Care 2014;23:3-14. [DOI: 10.1111/ecc.12093]

\section{Cramp 2012}

Cramp F, Byron-Daniel J. Exercise for the management of cancer-related fatigue in adults. Cochrane Database of Systematic Reviews 2012, Issue 11. [DOI: 10.1002/14651858.CD006145.pub3]

\section{Curt 2000}

Curt GA, Breibart W, Cella D, Groopman JE, Horning SJ, Itri LM, et al. Impact of cancer-related fatigue on the lives of patients: new findings from the fatigue coalition. The Oncologist 2000;5:353-60. [DOI: 10.1634/theoncologist.5-5-353]

\section{Custers 2016}

Custers JA, Gielissen MF, Janssen SH, de Wilt JH, Prins JB. Fear of cancer recurrence in colorectal cancer survivors. Support Cancer Care 2016;24:555-62. [DOI: 10.1007/s00520-015-2808-4]

\section{Deeks 2017}

Deeks JJ, Higgins JP, Altman DG, editor(s), on behalf of the Cochrane Statistical Methods Group. Chapter 9: Analysing data and undertaking meta-analyses. In: Higgins JP, Churchill R, Chandler J, Cumpston MS, editor(s). Cochrane Handbook for Systematic Reviews of Interventions Version 5.2.0 (updated June 2017). The Cochrane Collaboration, 2017. Available from www.training.cochrane.org/handbook.

\section{Denlinger 2009}

Denlinger CS, Barsevick AM. The challenges of colorectal cancer survivorship. Journal of the National Comprehensive Cancer Network 2009;7(8):883-94. [DOI: 10.6004/jnccn.2009.0058]

\section{Denlinger 2011}

Denlinger C, Engstrom P. Colorectal cancer survivorship: movement matters. Cancer Prevention Research 2011;4(4):502-11. [DOI: 10.1158/1940-6207] 


\section{DerSimonian 1986}

DerSimonian R, Laird N. Meta-analysis in clinical trials. Controlled Clinical Trials 1986;7(3):177-88.

\section{Devin 2016a}

Devin J, Sax A, Hughes G, Jenkins D, Aitken J, Chambers S, et al. The influence of high-intensity compared with moderateintensity exercise training on cardiorespiratory fitness and body composition in colorectal cancer survivors: a randomised control trial. Journal of Cancer Survivorship 2016;10(3):467-79. [DOI: 10.1007/s11764-015-0490-7]

\section{El-Shami 2015}

El-Shami K, Oeffinger KC, Erb NL, Willis A, Bretsch JK, PrattChampman ML, et al. American Cancer Society colorectal cancer survivorship care guidelines. A Cancer Journal for Clinicians 2015;65(6):427-55. [DOI: 10.3322/caac.21286]

\section{Endnote 2016 [Computer program]}

Endnote. Version X8. Philadelphia: Clarivate Analytics, 2016.Available at endnote.com.

\section{Fairey 2003}

Fairey AS, Courneya KS, Field CJ, Bell GJ, Jones LW, Mackey JR. Effects of exercise training on fasting insulin, insulin resistance, insulin-like growth factors, and insulin-like growth factor binding proteins in postmenopausal breast cancer survivors: a randomized controlled trial. Cancer Epidemiology Biomarkers \& Prevention 2003;12(8):721-7.

\section{Furmaniak 2016}

Furmaniak A, Menig M, Markes M. Exercise for women receiving adjuvant therapy for breast cancer. Cochrane Database of Systematic Reviews 2016, Issue 9. [DOI: 10.1002/14651858.CD005001.pub3]

\section{Galvao 2005}

Galvao DA, Newton RU. Review of exercise intervention studies in cancer patients. Journal of Clinical Oncology 2005;23(4):899-909. [DOI: 10.1200/JCO.2005.06.085]

\section{Giovannucci 2000}

Giovannucci E, Pollak MN, Platz EA, Willett WC, Stampfer MJ, Majeed N, et al. A prospective study of plasma insulin-like growth factor-1 and binding protein-3 and risk of colorectal neoplasia in women. Cancer Epidemiology, Biomarkers and Prevention 2000;9(4):345-9.

\section{GLOBOCAN 2018}

International Agency for Research on Cancer. Estimated age-standardized incidence and mortality rates (World) in 2018, worldwide, both sexes, all ages. gco.iarc.fr (accessed 21 December 2018).

\section{GRADE Working Group 2004}

GRADE Working Group. Grading quality of evidence and strength of recommendations. BMJ 2004;7454:1490-4. [DOI: https://doi.org/10.1136/bmj.328.7454.1490]

\section{GRADEpro GDT 2015 [Computer program]}

McMaster University (developed by Evidence Prime) GRADEpro GDT. Hamilton (ON): McMaster University (developed by Evidence Prime), 2015.Available at gradepro.org.

\section{Green 2002}

Green RJ, Metlay JP, Propert K, Catalano PJ, MacDonald JS, Mayor RJ, et al. Surveillance for second primary colorectal cancer after adjuvant chemotherapy: an analysis of intergroup 0089. Annals of Internal Medicine 2002;136(4):261-9. [DOI: 10.7326/0003-4819-136-4-200202190-00005]

\section{Haggar 2009}

Haggar FA, Boushery RP. Colorectal cancer epidemiology: incidence, mortality, survival, and risk factors. Clinics in Colon and Rectal Surgery 2009;22(4):191-7. [DOI: 10.1055/ s-0029-1242458]

\section{Haydon 2006}

Haydon A, Maclnnis R, English D, Giles G. Effect of physical activity and body size on survival after diagnosis with colorectal cancer. Gut 2006;55:62-7. [DOI: 10.1136/gut.2005.068189]

\section{Higgins 2003}

Higgins JP, Thompson SG, Deeks JJ, Altman DG. Measuring inconsistency in meta-analyses. BMJ 2003;327:557-60.

\section{Higgins 2011a}

Higgins JP, Altman DG, Gøtzsche PC, Jüni P, Moher D, Oxman AD, et al. The Cochrane Collaboration's tool for assessing risk of bias in randomised trials. $B M J$ 2011;343(7829):d5928. [DOI: 10.1136/bmj.d5928]

\section{Higgins 2011b}

Higgins JP, Deeks JJ, Altman DG, editor(s). Chapter 16: Special topics in statistics. In: Higgins JP, Green S, editor(s). Cochrane Handbook for Systematic Reviews of Interventions Version 5.1.0 (updated March 2011). The Cochrane Collaboration, 2011. Available from handbook.cochrane.org.

\section{Higgins 2017}

Higgins JP, Altman DG, Sterne JA, editor(s). Chapter 8: Assessing risk of bias in included studies. In: Higgins JP, Churchill R, Chandler J, Cumpston MS, editor(s). Cochrane Handbook for Systematic Reviews of Interventions version 5.2.0 (updated June 2017), Cochrane, 2017. Available from www.training.cochrane.org/handbook.

\section{Hong 2014}

Hong SK, Kim E-J, Chung SS, Kim KH, Lee R-A. Psychological attitude to self-appraisal of stoma patients: prospective observation of stoma duration effect to self appraisal. Annals of Surgical Treatment and Research 2014;86(3):152-60. [DOI: doi.org/10.4174/astr.2014.86.3.152]

\section{Howlader 2016}

Howlader N, Noone AM, Krapcho M, Miller D, Bishop K, Altekruse SF. SEER Cancer Statistics Review, 1975-2013. Bethesda (MD): National Cancer Institute 2016. 


\section{Hursting 2010}

Hursting SD, Berger NA. Energy balance, host-related factors, and cancer progression. Journal of Clinical Oncology 2010;28(26):4058-65. [DOI: 10.1200/JCO.2010.27.9935]

\section{Knols 2005}

Knols R, Aaronson NK, Uebelhart D, Fransen J, Aufdemkampe G. Physical exercise in cancer patients during and after medical treatment: a systematic review of randomized and controlled clinical trials. Journal of Clinical Oncology 2005;23(16):3830-42. [DOI: 10.1200/JCO.2005.02.148]

\section{Labianca 2010}

Labianca R, Nordlinger B, Beretta GD, Brouquet A, Cervantes A. Primary colon cancer: ESMO Clinical Practice Guidelines for diagnosis, adjuvant therapy and follow-up. Annals of Oncology 2010;21(5):70-7. [DOI: 10.1093/annonc/mdq168]

\section{Lassen 2009}

Lassen K, Soop M, Nygren J, Cox BW, Hendry PO, Spies C, et al. Consensus review of optimal perioperative care in colorectal surgery. Enhanced recovery after surgery (ERAS) group recommendations. Archives of Surgery 2009;144(10):961-9. [DOI: 10.1001/archsurg.2009.170]

\section{Leitzmann 2015}

Leitzmann M, Powers H, Anderson A, Scoccianti C, Berrino F, Boutron-Ruault M, et al. European Code against Cancer 4th edition: physical activity and cancer. Cancer Epidemiology 2015;39S:46-55. [DOI: 10.1016/j.canep.2015.03.009]

\section{Lin 2009}

Lin J, Brimmer D, Maloney E, Nyarko E, Belue R, Reeves WC. Further validation of the Multidimensional Fatigue Inventory in a US adult population sample. Population Health Metrics 2009;7(18):1-12. [DOI: 10.1186/1478-7954-7-18]

\section{Lynch 2008}

Lynch BM, Cerin E, Owen N, Hawkes AL, Aitken JF. Prospective relationships of physical activity with quality of life among colorectal cancer survivors. Journal of Clinical Oncology 2008;26(27):4480-7. [DOI: 10.1200/JCO.2007.15.7917]

\section{Lynch 2010}

Lynch BM, Owen N, Hawkes AL, Aitken JF. Perceived barriers to physical activity for colorectal cancer survivors. Support Cancer Care 2010;18:729-34. [DOI: 10.1007/s00520-009-0705-4]

\section{Markle 2010}

Markle B, May EJ, Majumdar APN. Do nutraceutics play a role in the prevention and treatment of colorectal cancer? Cancer Metastasis Reviews 2010;29:395-404. [DOI: 10.1007/ s10555-010-9234-3]

\section{Meyerhardt 2006}

Meyerhardt J, Heseltine D, Neidzwiechi D, Hollis D, Saltz L, Mayer R, et al. Impact of physical activity on cancer recurrence and survival in patients with stage III colon cancer: findings from CALGB 89803. Journal of Clinical Oncology 2006;24(22):3535-41. [DOI: 10.1200/JCO.2006.06.0863]

\section{Meyerhardt 2009}

Meyerhardt JA, Giovannucci EL, Ogino S, Kirkner GJ, Chan AT, Willett W. Physical activity and survival in male colorectal cancer survivors. Archives of Internal Medicine 2009;169(22):2102-08. [DOI: 10.1001/archinternmed.2009.412.]

\section{Mishra 2012a}

Mishra SI, Scherer RW, Synder C, Geige PM, Berlanstein DR, Topaloglu O. Exercise interventions on health-related quality of life for people with cancer during active treatment. Cochrane Database of Systematic Reviews 2012, Issue 8. [DOI: 10.1002/14651858.CD008465.pub2]

\section{Mishra 2012b}

Mishra SI, Scherer RW, Geigle PM, Berlanstein DR, Topaloglu O, Gotay CC. Exercise interventions on health-related quality of life for cancer survivors. Cochrane Database of Systematic Reviews 2012, Issue 8. [DOI: 10.1002/14651858.CD007566.pub2]

\section{Moher 2009}

Moher D, Liberati A, Tetzlaff J, Altman DG, PRISMA Group. The PRISMA Group (2009). Preferred Reporting Items for Systematic Reviews and Meta-Analyses: The PRISMA Statement. BMJ 2009;339(b2535):1-6. [DOI: 10.1136/bmj.b2535]

\section{Mosher 2016}

Mosher CE, Winger JG, Given BA, Helft PR, O'Neill BH. Mental health outcomes during colorectal cancer survivorship: a review of the literature. Psycho-Oncology 2016;25:1261-70. [DOI: 10.1002/pon.3954]

\section{NCCN 2016}

National Comprehensive Cancer Network (NCCN). Clinical practice guidelines in oncology: cancer related fatigue. www.nccn.org/professionals/physician_gls/pdf/fatigue.pdf (accessed 13 February 2017).

\section{Ouakrim 2015}

Ouakrim DA, Pizot C, Boniol M, Malvezzi M, Boniol M, Negri E, et al. Trends in colorectal cancer mortality in Europe: retrospective analysis of the WHO mortality database. BMJ 2015;351(h4970):1-10. [DOI: 10.1136/bmj.h4970]

\section{Peel 2009}

Peel B, Sui X, Matthews C, Adams S, Hebert J, Hardin J, et al. Cardiorespiratory fitness and digestive cancer mortality: findings from the Aerobics Center Longitudinal Study (ACLS). Cancer Epidemiology, Biomarkers and Prevention 2009;18(4):1111-17. [DOI: 10.1158/1055-9965.EPI-08-0846]

\section{Pereira 2012}

Pereira MG, Figueiredo AP, Fincham FD. Anxiety, depression, traumatic stress and quality of life in colorectal cancer after different treatments: a study with Portuguese patients and their partners. European Journal of Nursing Oncology 2012;16(3):227-32. [DOI: 10.1016/j.ejon.2011.06.006]

\section{Prado 2008}

Prado CM, Leiffers JR, McCargar LJ, Reiman T, Sawyer MB, Martin L, et al. Prevalence and clinical implications of sarcopenic obesity in patients with solid tumours of the 
respiratory and gastrointestinal tracts: a population-based study. Lancet Oncology 2008;9:629-35. [DOI: 10.1016/ S1470-2045(08)70153-0]

\section{Quadrilatero 2003}

Quadrilatero J, Hoffman-Geotz L. Physical activity and colon cancer: a systemic review of potential mechanisms. Journal of Sports Medicine and Physical Fitness 2003;43(2):121-38.

\section{Ramsey 2002}

Ramsey SD, Berry K, Moinpour C, Giedzinska A, Anderson MR. Quality of life in long term survivors of colorectal cancer. American Journal of Gastroenterology 2002;97(5):1228-34. [DOI: $10.1111 / \mathrm{j} .1572-0241.2002 .05694 . x]$

\section{Review Manager 2014 [Computer program]}

Nordic Cochrane Centre, The Cochrane Collaboration Review Manager 5 (RevMan 5). Version 5.3. Copenhagen: Nordic Cochrane Centre, The Cochrane Collaboration, 2014.

\section{Robsahm 2013}

Robsahm TE, Aagnes B, Hjartåker A, Langseth H, Bray Fl, Larsen IK. Body mass index, physical activity, and colorectal cancer by anatomical sub sites: a systematic review and meta-analysis of cohort studies. European Journal of Cancer Prevention 2013;22(6):492-505. [DOI: 10.1097/ CEJ.0b013e328360f434]

\section{Rock 2012}

Rock CL, Doyle C, Demrk-Wahnefried W, Meyerhardt J, Courneya $\mathrm{K}$, Schwartz AL, et al. Nutrition and physical activity guidelines for cancer survivors. CA: A Cancer Journal for Clinicians 2012;62:242-74. [DOI: 10.3322/caac.21142]

\section{Ryerson 2016}

Ryerson AB, Eherman CR, Alterkruse SF, Ward JW, Jemal A, Sherman RL, et al. Annual report to the nation on the status of cancer, 1975-2012, featuring the increasing incidence of liver cancer. Cancer 2016;122(9):1312-37. [DOI: 10.1002/cncr.29936]

\section{Samad 2005}

Samad AK, Taylor RS, Marshall T, Chapman MAS. A metaanalysis of the association of physical activity with reduced risk of colorectal cancer. Colorectal Disease 2005;7(3):204-13. [DOI: 10.1111/j.1463-1318.2005.00747.x]

\section{Schmid 2015}

Schmid D, Leitzmann MF. Cardiorespiratory fitness as predictor of cancer mortality: a systematic review and meta-analysis. Annals of Oncology 2015;26(2):272-8. [DOI: 10.1093/annonc/ mdu250]

\section{Schmitz 2005}

Schmitz KH, Holtzman J, Courneya KS, Masse LC, Duval S, Kane R. Controlled physical activity trials in cancer survivors: a systematic review and meta-analysis. Cancer Epidemiology, Biomarkers and Prevention 2005;14(7):1588-95. [DOI: 10.1158/1055-9965.EPI-04-0703]

\section{Schmitz 2010}

Schmitz KH, Courneya K, Matthews C, Demark-Wahnefried W, Galvao DA, Pinto BM, et al. American College of Sports Medicine roundtable on exercise guidelines for cancer survivors. Medicine and Science in Sports and Exercise 2010;42(7):1409-26. [DOI: 10.1249/MSS.0b013e3181e0c112]

\section{Schneider 2007}

Schneider EC, Malin JL, Kahn KL, Ko CY, Adam J, Epstein AM. Surviving colorectal cancer: patient-reported symptoms 4 years after diagnosis. Cancer 2007;110:2075-82. [DOI: 10.1002/ cncr.23021]

\section{Schroeder 1991}

Schroeder D, Hill DL. Postoperative fatigue: a prospective physiological study of patients undergoing major abdominal surgery. Australian and New Zealand Journal of Surgery 1991;61(10):774-9. [DOI: 10.1111/j.1445-2197.1991.tb00149.x]

\section{Schumacher 2017}

Schumacher A. Validation of the six-minute walk test for predicting peak oxygen consumption cancer survivors [Master of Science]. Colorado (USA): School of Sport and Exercise Science, 2017.

\section{Slattery 2003}

Slattery ML, Edwards S, Curtin K, Ma K, Edwards R, Holubkov R, et al. Physical activity and colorectal cancer. American Journal of Epidemiology 2003;158(3):214-24. [DOI: 10.1093/aje/kwg134]

\section{Speck 2010}

Speck RM, Courneya KS, Masse LC, Duval S, Schmitz KH. An update of controlled physical activity trials in cancer survivors: a systematic review and meta-analysis. Journal of Cancer Survivorship 2010;4:87-100. [DOI: 10.1007/s11764-009-0110-5]

\section{Sterne 2017}

Sterne JA, Egger M, Moher D, Boutron I, editor(s). Chapter 10: Addressing reporting biases. In: Higgins JP, Churchill R, Chandler J, Cumpston MS, editor(s). Cochrane Handbook for Systematic Reviews of Interventions Version 5.2.0 (updated June 2017). The Cochrane Collaboration, 2017. Available from www.training.cochrane.org/handbook.

\section{Stewart 2014}

Stewart BW, Wild CP, editor(s). World Cancer Report. Vol. 3. Lyon: International Agency for Research on Cancer, 2014.

\section{Stone 2008}

Stone PC, Minto O. Cancer-related fatigue. European Journal of Cancer 2008;44(8):1097-104. [DOI: 10.1016/j.ejca.2008.02.037]

\section{Sullivan 2011}

Sullivan R, Peppercorn J, Sikora K, Zalcberg J, Meropal NJ, Amir E, et al. Delivering affordable cancer care in high-income countries. Lancet Oncology 2011;12(10):933-80. [DOI: 10.1016/ S1470-204] 


\section{Thomas 2014}

Thomas RJ, Holm H, Al-Adhami A. Physical activity after cancer: an evidence review of the international literature. British Journal of Medical Practitioners 2014;7(1):16-22.

\section{Tierney 2007}

Tierney JF, Stewart LA, Ghersi D, Burdett S, Sydes MR. Practical methods for incorporating summary time-toevent data into meta-analysis. Trials 2007;8(16):1-16. [DOI: $10.1186 / 1745-6215-8-16]$

\section{Vassbakk-Brovold 2016}

Vassbakk-Brovold K, Kersten C, Fergan L, Mjaland O, Mjaland S, Seiler S, et al. Cancer patients participating in a lifestyle intervention during chemotherapy greatly over-report their physical activity level: a validation study. BMC Sports Science Medicine and Rehabilitation 2016;8(10):1-9. [DOI: 10.1186/ s13102-016-0035-z]

\section{Wagner 2004}

Wagner LI, Cella D. Fatigue and cancer: causes, prevalence and treatment approaches. British Journal of Cancer 2004;91:822-8. [DOI: 10.1038/sj.bjc.6602012]

\section{Wang 2017}

Wang N, Khankari NK, Cai H, Li H, Yang G, Gao Y. Prediagnosis body mass index and waist-hip circumference ratio in association with colorectal cancer survival. International Journal of Cancer 2017;140:292-301. [DOI: 10.1002/ijc.30459]

\section{West 2014a}

West MA, Loughney L, Barben CP, Sripadam R, Kemp GJ, Grocott MP, et al. The effects of neoadjuvant chemo radiotherapy on physical fitness and morbidity in rectal cancer surgery patients. European Journal of Surgical Oncology 2014;40(11):1421-8. [DOI: 10.1016/j.ejso.2014.03.021]

\section{CHARACTERISTICS OF STUDIES}

Characteristics of included studies [ordered by study ID]

\section{West 2014b}

West MA, Lythgoe D, Barben CP, Noble L, Kemp GJ, Jack S, et al. Cardiopulmonary exercise variables are associated with post operative morbidity after major colonic surgery: a prospective blinded observational study. British Journal of Anaesthesia 2014;112(4):665-71. [DOI: 10.1093/bja/aet408]

\section{West 2014c}

West MA, Parry MG, Lythgoe D, Barben CP, Kemp GJ, Grocott MP, et al. Cardiopulmonary exercise testing for the prediction of morbidity risk after rectal cancer surgery. British Journal of Surgery 2014;101(9):1166-72. [DOI: 10.1002/bjs.9551]

\section{Wolin 2009}

Wolin KY, Yan Y, Colditz GA, Lee I-M. Physical activity and colon cancer prevention: a meta-analysis. British Journal of Cancer 2009;100:611-16. [DOI: 10.1038/sj.bjc.6604917]

\section{Yost 2008}

Yost KJ, Hahn EA, Zaslavsky AM, Ayanian JZ, West DW. Predictors of health-related quality of life in patients with colorectal cancer. Health and Quality of Life Outcomes 2008;6(1):66. [DOI: 10.1186/1477-7525-6-66]

\section{References to other published versions of this review}

\section{McGettigan 2017}

McGettigan M, Cardwell C, Cantwell MM, Tully MA. Physical activity and exercise interventions for disease-related physical and mental health during and following treatment in people with non-advanced colorectal cancer. Cochrane Database of Systematic Reviews 2017, Issue 11. [DOI: 10.1002/14651858.CD012864]

* Indicates the major publication for the study

Bourke 2011

\section{Study characteristics}

\begin{tabular}{|c|c|}
\hline \multirow[t]{9}{*}{ Methods } & Study design: RCT \\
\hline & Study location: UK \\
\hline & Inclusion criteria: \\
\hline & $\begin{array}{l}\text { 1. patients who had histologically confirmed colon cancer (Dukes stages A-C) resected } 6 \text { to } 24 \text { months } \\
\text { previously }\end{array}$ \\
\hline & Exclusion criteria: \\
\hline & $\begin{array}{l}\text { 1. existing participation in regular physical activity (purposeful activity of at least a moderate-intensity } \\
\text { of } 30 \text { minutes or more, } 3 \text { times a week) }\end{array}$ \\
\hline & 2. a Karnofsky rating of less than 80 \\
\hline & 3. unstable angina \\
\hline & 4. uncontrolled hypertension \\
\hline
\end{tabular}

Physical activity interventions for disease-related physical and mental health during and following treatment in people with non- 
Baseline imbalances: not reported

Withdrawals and exclusions: 1 dropout in intervention group (stroke)

Age, mean (SD) - total: 69 years (range 52-80 years); control: 70.3 (8.7) years; intervention: 67.9 (5.7) years

Gender - total; 12 male, 6 female; control: 7 male, 2 female; intervention; 5 male, 4 female

Ethnicity: not reported

Comorbidities: not reported

SES: not reported

Cancer type: colon

Colorectal cancer stage: Dukes A-C

Type of treatment n: surgery: 8 control, 9 intervention; chemotherapy: 2 control, 4 intervention; palliative care: 1 control, 0 intervention

Receiving or finished treatment: finished

Time beyond treatment (months): 16.7 control; 16.4 intervention

Time since diagnosis: not reported

\section{Physical activity description}

Frequency: 2 group-based supervised sessions weekly and one home-based Intensity: $55 \%$ to $85 \%$ of age predicted maximum heart rate or rate of perceived exertion between 11 and 15

Time: 30 minutes of aerobic exercise per session, 2 and 4 sets of 8 to 12 repetitions of resistance exercises

Type: aerobic (treadmills, rowing ergometers, and cycling ergometers) and resistance Volume: not reported Progression: during the final 6 weeks of the intervention, participants attended the university facility once a week and were asked to perform two home-based sessions a week

Length of intervention: 12 weeks

Control group: usual care

Setting: exercise suite in university and home-based

Supervised or self-directed: both

Co-interventions: dietary advice information pack, on a fortnightly basis throughout the intervention, engaged in healthy eating seminars in a group format, lasting approximately 15 to 30 minutes

2. (Macro/micronutrient outcomes (three-day diet diaries)

3. Fatigue (FACT-F, isometric fatigue protocol was also administered at $20 \%$ of maximum voluntary torque with simultaneous assessment of motor unit recruitment using sEMG)

4. QoL (FACT-C)

5. Aerobic exercise tolerance (Bruce Protocol Treadmill Test)

6. Functional capacity (sit to stand test) 
7. Lower-limb strength (isokinetic dynamometry)

Time points measured: baseline and 12 weeks

Time points reported: baseline and 12 weeks

Adverse events: a stroke occurred in the intervention group

\begin{tabular}{ll}
\hline Notes & Funding source: not reported \\
\hline
\end{tabular}

\section{Risk of bias}

\begin{tabular}{|c|c|c|}
\hline Bias & Authors' judgement & Support for judgement \\
\hline $\begin{array}{l}\text { Random sequence genera- } \\
\text { tion (selection bias) }\end{array}$ & Low risk & Randomised via code numbers using nQuery statistical software \\
\hline $\begin{array}{l}\text { Allocation concealment } \\
\text { (selection bias) }\end{array}$ & Unclear risk & Not described \\
\hline $\begin{array}{l}\text { Blinding of participants } \\
\text { and personnel (perfor- } \\
\text { mance bias) } \\
\text { All outcomes }\end{array}$ & High risk & $\begin{array}{l}\text { Not possible to blind participants and personnel due to the nature of the inter- } \\
\text { vention }\end{array}$ \\
\hline $\begin{array}{l}\text { Blinding of outcome as- } \\
\text { sessment (detection bias) } \\
\text { All outcomes }\end{array}$ & Low risk & $\begin{array}{l}\text { An exercise physiologist who was blinded to group allocation assessed out- } \\
\text { comes. }\end{array}$ \\
\hline $\begin{array}{l}\text { Incomplete outcome data } \\
\text { (attrition bias) } \\
\text { All outcomes }\end{array}$ & Low risk & $\begin{array}{l}\text { Retention as high, attrition (5\%) and exclusions were reported with reasons. } \\
\text { ITT analysis conducted }\end{array}$ \\
\hline $\begin{array}{l}\text { Selective reporting (re- } \\
\text { porting bias) }\end{array}$ & Low risk & There appears to be no selective reporting of outcomes. \\
\hline Other bias & Unclear risk & Baseline characteristics are presented, baseline imbalances are not. \\
\hline
\end{tabular}

\section{Brown 2017}

\section{Study characteristics}

Study design: RCT
Study location: USA
Inclusion criteria:
1. histologically confirmed stage I-III colon cancer
2. completed adjuvant therapy
3. age $\geq 18$ years
4. written physician approval
5. the ability to walk unaided for six minutes

\section{Exclusion criteria:}

Physical activity interventions for disease-related physical and mental health during and following treatment in people with non- 
Brown 2017 (Continued)

1. history of another primary cancer (other than non-melanoma skin cancer)

2. evidence of metastatic colon cancer

3. planning to receive any additional adjuvant chemotherapy or surgery (i.e. ostomy reversal); pregnant

or breast feeding

4. unable to provide baseline blood sample

5. cardiac conditions

6. any other condition which, may impede testing of the study hypothesis or make it unsafe to engage in the exercise programme

Participants

Number randomised: 39; 13 to usual care, 14 to low-dose exercise (150 minutes), 12 to high-dose exercise (300 minutes)

Baseline imbalances: no significant differences at baseline

Withdrawals and exclusions: 38/39 participants completed study (1 lost to follow-up in usual care control group)

Age, mean (SD):

Total; 56.5 (10.0) years

Usual care; 57.9 (9.7)

Low dose; 58.2 (9.8)

High dose; 53.1 (10.5)

Gender, n (\%):

Total; Male and female (57\%)

Control; male 4(31\%), female $9(69 \%)$

Low dose; Male 7 (50\%), female 7 (50\%)

High dose; Male (33\%), female 8 (67\%)

Ethnicity: Majority white (80)\%

Comorbidities, n (\%): hypertension 13 (33\%), hyperlipidaemia 6 (15\%), T2DM 5 (13\%), CVD 4 (10\%)

SES: not reported

Cancer type: colon

Cancer stage, $\mathrm{n}(\%)$ :

Stage I; 5 (13\%)

Stage II; $14(36 \%)$

Stage III; 20 (51\%)

Type of treatment: surgical resection and adjuvant chemotherapy

Receiving or finished treatment: finished

Time beyond treatment, mean (SD) months: Total; 10.9 (6.1) months

(less than or equal to 12 months $25(64 \%)$ greater than 12 months $14(36 \%)$ )

Time since diagnosis: not reported

\section{Physical activity description}

Frequency: not reported

Physical activity interventions for disease-related physical and mental health during and following treatment in people with non- 
Brown 2017 (Continued)

Intensity: moderate-intensity ( $70.7 \%$ of age predicted maximum heart rate)

Time: the initial exercise dose prescribed in week one of the study is $60 \mathrm{~min}$

Type: home-based treadmill walking

Progression: low-dose and high-dose exercise groups increase their exercise to $150 \mathrm{~min}$ in week 1 and

$300 \mathrm{~min}$ in week 1, respectively. Exercise increased by $30 \mathrm{~min} /$ week 1 as the participant successfully re-

sponds to the exercise dose prescribed in the prior week

Length of intervention: 6 months

Control group: maintained their pre- study levels of physical activity and/or followed the recommendations provided by their physician

Setting: home-based

Supervised or self-directed: self-directed

Co-interventions: none

Outcomes
1. Physical activity (accelerometry)
3. Anthropometric
4. Clinical outcomes
5. Quality of Life
6. Biomarkers including soluble intercellular adhesion molecule-1 (sICAM-1) and soluble vascular adhe-
sion molecule-1 (sVCAM-1) prognostic biomarkers

Time points measured: baseline, six months

Time points reported: baseline, six months

Adverse events: graded using the Common Terminology Criteria for Adverse Events version 4.0

Notes

Funding source: National Cancer Institute and the National Center for Advancing Translational Science

\section{Risk of bias}

\begin{tabular}{lll}
\hline Bias & Authors' judgement & Support for judgement \\
\hline $\begin{array}{l}\text { Random sequence genera- } \\
\text { tion (selection bias) }\end{array}$ & Unclear risk & Not described \\
\hline $\begin{array}{l}\text { Allocation concealment } \\
\text { (selection bias) }\end{array}$ & Unclear risk & Not described \\
\hline $\begin{array}{l}\text { Blinding of participants } \\
\text { and personnel (perfor- } \\
\text { mance bias) }\end{array}$ & High risk & $\begin{array}{l}\text { Not possible to blind participants and personnel due to the nature of the inter- } \\
\text { vention }\end{array}$ \\
\hline $\begin{array}{l}\text { Blinding of outcome as- } \\
\text { sessment (detection bias) } \\
\text { All outcomes }\end{array}$ & Low risk & $\begin{array}{l}\text { Outcome measures were obtained by assessors blinded to treatment assign- } \\
\text { ment }\end{array}$ \\
\hline $\begin{array}{l}\text { Incomplete outcome data } \\
\text { (attrition bias) } \\
\text { All outcomes }\end{array}$ & Low risk & $\begin{array}{l}\text { One participant did not provide end point data (97\% follow-up rate) (3\% attri- } \\
\text { tion) }\end{array}$ \\
\hline $\begin{array}{l}\text { Selective reporting (re- } \\
\text { porting bias) }\end{array}$ & High risk & $\begin{array}{l}\text { Study protocol included outcomes (dietary intake, cardiopulmonary and QoL) } \\
\text { data that are not presented in available reports }\end{array}$ \\
\hline
\end{tabular}


Brown 2017 (Continued)

Other bias Low risk The study appears to be free of other problems that could put it at a high risk of bias

\section{Study characteristics}

Study design: RCT
Study location: Spain
Inclusion criteria:
1. more than 18 years old
2. received curative treatment due to cancer (surgery, chemotherapy and/or radiotherapy)
3. diagnosed with grades I to III A of colorectal cancer
4. completed coadjuvant treatment
Exclusion criteria:
1. presented cancer recurrence
2. underwent previous abdominal surgeries
3. were diagnosed with concomitant conditions, such as previous lower-back pain or musculoskeletal
conditions (e.g. osteoarthritis, fibromyalgia or chronic fatigue syndrome)

Participants

Number randomised:46; 23 to the intervention group and 23 patients to the usual care group

Baseline imbalances: no significant differences at baseline

Withdrawals and exclusions: six dropouts ( 2 in intervention group, 4 in usual care group)

Age, mean (SD) years:

60 years (usual care: 62.3 (7.9) years, intervention: 57.5 (8.0) years)

Gender, n (\%):

Total: 26 males, 14 females

usual care: male 13 (68.4\%), female 8 (31.6\%)

Intervention: male 13 (61.9\%) female 6 (38.1\%)

Ethnicity: not reported

Comorbidities: not reported

SES: University degree, $\mathrm{n}(\%)$

Usual care $2(10.5 \%)$

Intervention 6 (28.6\%)

Cancer type: colon

Cancer stage n (\%):

stage II 14; usual care $7(36.8 \%)$, intervention $=7(33.3 \%)$

stage IIla 26; usual care 12 (63.2\%), intervention 14 (66.7\%)

Type of treatment: surgery, other treatment not reported

Physical activity interventions for disease-related physical and mental health during and following treatment in people with non- 
Cantarero-Villanueva 2016 (Continued)

Receiving or completed treatment: finished

Time beyond treatment, mean (SD): 13.3 months

Usual care $=14.6(10)$ Months

Intervention $=12(7.4)$ months

Time since diagnosis: mean (SD): 15.4 months

Usual care: 17.1 (11.3) months

Intervention: 13.8 (7.8) months

\section{Physical activity description}

Frequency: 3 times per week

Intensity: not reported

Time: 90 minutes

Type: core stabilisation and stretching exercises

Volume, progression $=1$ set of 8 - 10 reps to 2 sets of 10 reps

Length of intervention: 8 weeks

Control group: usual care, including written general recommendations for a healthy lifestyle

Setting: activity centre

Supervised or self-directed: supervised

Co-interventions: none

\begin{tabular}{|c|c|c|}
\hline \multirow[t]{4}{*}{ Outcomes } & \multicolumn{2}{|c|}{$\begin{array}{l}\text { 1. Physical fitness/physical function: abdominal isometric endurance (trunk-curl test), flexibility ("chair } \\
\text { sit and reach test"), functional capacity (6-min walk test with treadmill), static balance (Flamingo Test), } \\
\text { fitness perceived (International Fitness Scale), lower back muscle strength (dynamometry) } \\
\text { 2. Fatigue (Piper Fatigue Scale) } \\
\text { 3. Body composition (bioelectrical impedance analysis) } \\
\text { 4. Pain (Brief Pain Inventory) } \\
\text { 5. Quality of life } \\
\text { 6. Pressure Pain Thresholds } \\
\text { 7. Muscle structure }\end{array}$} \\
\hline & \multicolumn{2}{|c|}{ Time points measured: baseline, 8 weeks and 6 -month follow-up } \\
\hline & \multicolumn{2}{|c|}{ Time points reported: baseline 8 weeks and 6-month follow-up } \\
\hline & \multicolumn{2}{|c|}{ Adverse events: reported } \\
\hline Notes & \multicolumn{2}{|c|}{ Funding source: Universidad de Granada } \\
\hline \multicolumn{3}{|l|}{ Risk of bias } \\
\hline Bias & Authors' judgement & Support for judgement \\
\hline $\begin{array}{l}\text { Random sequence genera- } \\
\text { tion (selection bias) }\end{array}$ & Low risk & Computer-generated numbers \\
\hline $\begin{array}{l}\text { Allocation concealment } \\
\text { (selection bias) }\end{array}$ & Low risk & $\begin{array}{l}\text { Sequence created was introduced in numbered, opaque, sealed envelopes by } \\
\text { an external researcher who did not participate in the study }\end{array}$ \\
\hline $\begin{array}{l}\text { Blinding of participants } \\
\text { and personnel (perfor- } \\
\text { mance bias) }\end{array}$ & High risk & $\begin{array}{l}\text { Not possible to blind participants and personnel } \\
\text { due to the nature of the intervention }\end{array}$ \\
\hline
\end{tabular}

Physical activity interventions for disease-related physical and mental health during and following treatment in people with non- 
Cantarero-Villanueva 2016 (Continued)

All outcomes

$\begin{array}{lll}\text { Blinding of outcome as- } & \text { Low risk } & \begin{array}{l}\text { A member of the research group who was blinded to the patient group } \\ \text { sessment (detection bias) }\end{array} \\ \text { All outcomes } & \text { assessed the variables }\end{array}$

All outcomes

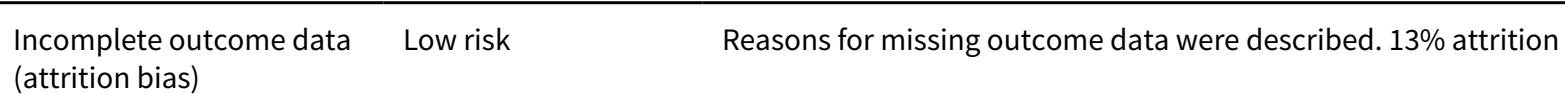

All outcomes

\begin{tabular}{lll}
\hline $\begin{array}{l}\text { Selective reporting (re- } \\
\text { porting bias) }\end{array}$ & High risk & $\begin{array}{l}\text { Study protocol included outcome data that are not presented in available re- } \\
\text { ports }\end{array}$ \\
\hline Other bias & Low risk & $\begin{array}{l}\text { The study appears to be free of other problems that could put it at a high risk } \\
\text { of bias }\end{array}$ \\
\hline
\end{tabular}

Courneya 2003

\section{Study characteristics}

Study design: RCT
Study location: Canada
Inclusion criteria:
1. surgery for colorectal cancer within the past 3 months
2. recovery from surgery as indicated by an attending physician
3. ability to understand and provide written informed consent in English
4. passed the revised Physical Activity Readiness Questionnaire (rPAR-Q; Thomas et al. 1992)
5. no contraindications to exercise as determined by a submaximal cardiorespiratory fitness test

Exclusion criteria: not reported

Participants

Number randomised: 102; 69 to the exercise group and 33 to a waiting list control group

Baseline imbalances: no significant differences at baseline

Withdrawals and exclusions: 9 lost to follow-up in total (7 in intervention group 2 in control group)

Age, mean (SD) years:

Total; 60.3 years

Control; 61.1 (9.93) years

Intervention; 59.92 (10.73) years

Gender:

Control: $64.5 \%$ male

Intervention: $54.8 \%$ male

Ethnicity: not reported

Comorbidities: not reported

SES:

Physical activity interventions for disease-related physical and mental health during and following treatment in people with non- 


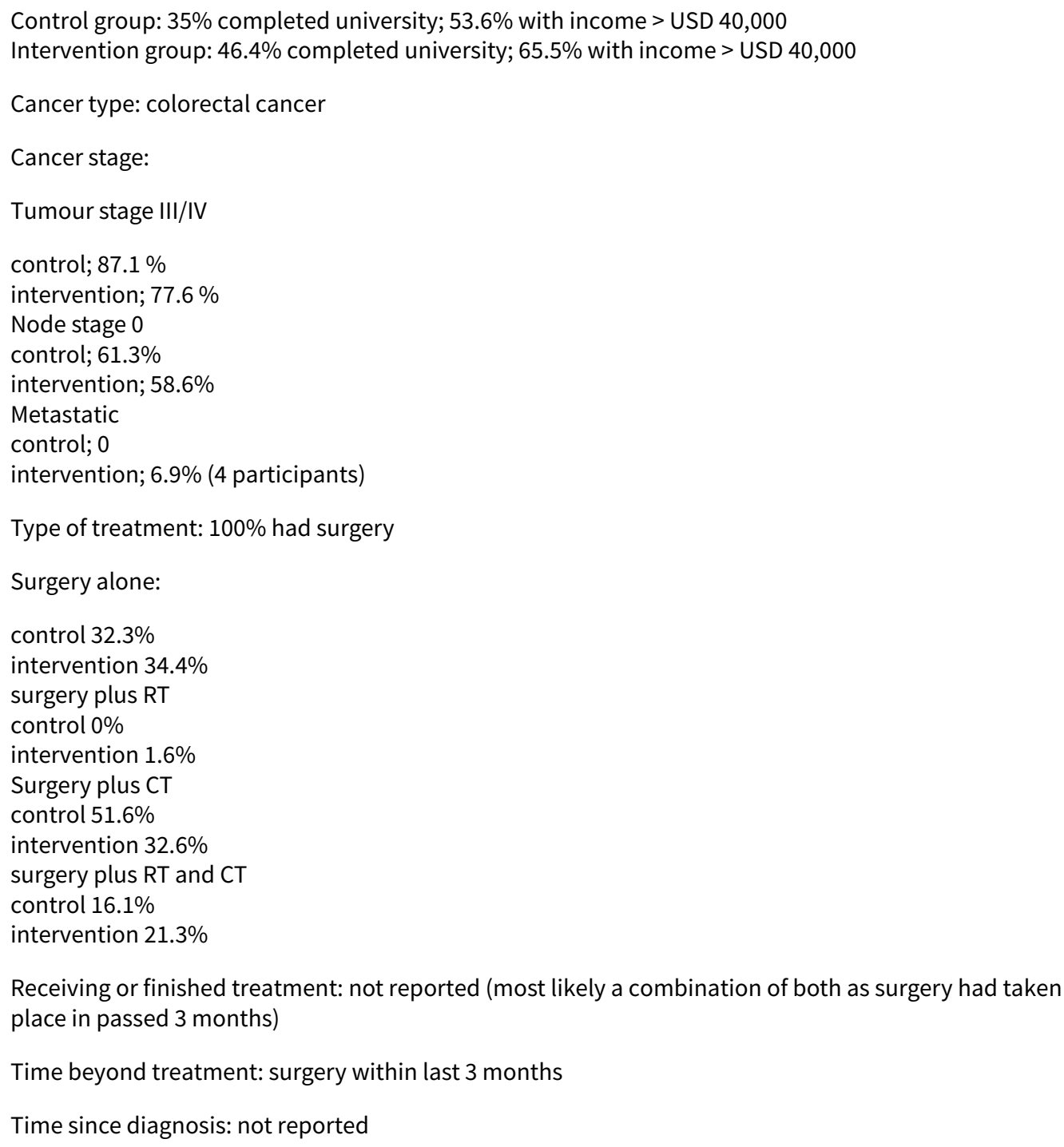

\section{Physical activity description}

Frequency: 3 to 5 times per week Intensity: to $65 \%$ to $75 \%$ of heart rate maximum

Time: 20 to 30 minutes

Type: participants were allowed to choose the mode of exercise they preferred (e.g. swimming, cycling) but if they had no preference they were prescribed walking

Length of intervention: 16 weeks

Total number of exercise sessions: 48 to 80

Control group: were asked not to initiate any structured exercise over the course of the intervention (wait list)

Setting: home based

Supervised or self-directed: self-directed

Co-interventions: none

Outcomes

1. Quality of life (FACT-C scale, FACT-G, TOI score- sum of physical and FWB subscale and colorectal subscale)

2. Satisfaction with life, measured (Satisfaction with Life scale)

3. Depression and anxiety, (CES-D scale and STAI, respectively)

Physical activity interventions for disease-related physical and mental health during and following treatment in people with non- 
Courneya 2003 (Continued)

4. Cardiovascular fitness (Modified Balke Treadmill Test)

5. Body composition (Harpenden callipers)

6. Flexibility, (sit and reach test)

Time points measured: baseline and 16 weeks

Time points reported: baseline and 16 weeks

Adverse events: not reported

Notes

Funding source: National Cancer Institute of Canada, the Alberta Heritage Foundation for Medical Research and Sociobehavioral Cancer Research Network

\section{Risk of bias}

\begin{tabular}{|c|c|c|}
\hline Bias & Authors' judgement & Support for judgement \\
\hline $\begin{array}{l}\text { Random sequence genera- } \\
\text { tion (selection bias) }\end{array}$ & Low risk & A random numbers table was used \\
\hline $\begin{array}{l}\text { Allocation concealment } \\
\text { (selection bias) }\end{array}$ & Unclear risk & Not described \\
\hline $\begin{array}{l}\text { Blinding of participants } \\
\text { and personnel (perfor- } \\
\text { mance bias) } \\
\text { All outcomes }\end{array}$ & High risk & $\begin{array}{l}\text { Not possible to blind participants and personnel due to the nature of the inter- } \\
\text { vention }\end{array}$ \\
\hline $\begin{array}{l}\text { Blinding of outcome as- } \\
\text { sessment (detection bias) } \\
\text { All outcomes }\end{array}$ & Low risk & Fitness test conducted by person blinded to the experimental group \\
\hline $\begin{array}{l}\text { Incomplete outcome data } \\
\text { (attrition bias) } \\
\text { All outcomes }\end{array}$ & Low risk & $\begin{array}{l}\text { Attrition and exclusions were reported, with reasons. Retention rate was } \\
91.2 \% \text {. } \\
\text { ITT analysis conducted }\end{array}$ \\
\hline $\begin{array}{l}\text { Selective reporting (re- } \\
\text { porting bias) }\end{array}$ & Low risk & There appears to be no selective reporting of outcomes \\
\hline Other bias & Low risk & $\begin{array}{l}\text { The study appears to be free of other problems that could put it at a high risk } \\
\text { of bias }\end{array}$ \\
\hline
\end{tabular}

\section{Courneya 2016}

\section{Study characteristics}

Study design: RCT
Study location: Canada and Australia
Inclusion criteria:
1. high risk stage II or stage III colon cancer
2. received adjuvant chemotherapy within the past 2-6 months
3. an Eastern Cooperative Oncology Group performance status of 0 or 1
4. are not currently meeting physical activity guidelines (i.e. the equivalent of 150 minutes/week of
moderate-intensity exercise)
5. able to complete at least two stages of a submaximal treadmill test

Physical activity interventions for disease-related physical and mental health during and following treatment in people with non- 


\section{Exclusion criteria: not reported}

Participants

Number randomised: $273 ; 136$ to a structured exercise programme (SEP) and 137 to health education materials (HEM)

Baseline imbalances: no significant differences at baseline

Withdrawals and exclusions: 62 excluded from primary analysis at year 1 (211 analysed; 105 in the control (HEM) group, 106 in the intervention group)

Age, $\mathrm{n}(\%)$ analysed group:

less than 65:

Control (HEM): 71 (68\%)

Intervention: 71 (67\%)

Gender, n (\%) analysed group:

Control (HEM) male; 46 (44\%), female 59 (56\%)

Intervention; male 46 (43\%), female 60 (57\%)

Ethnicity: not reported

Comorbidities: not reported

SES: not reported

Cancer type: colon

Cancer stage, $\mathrm{n}(\%)$ :

High risk stage II:

control (HEM): 12 (11\%)

Intervention: 14 (13\%)

Stage III:

control (HEM): 93 (89\%)

intervention: 92 (87\%)

Type of treatment: $100 \%$ received chemotherapy

Receiving or finished treatment: finished

Time beyond treatment: 2-6 months

Time since diagnosis, median (range):

Control; 12 (0-16) months

Intervention; 12 (8-17) months

Interventions

\section{Physical activity description:}

Frequency: biweekly face-to-face counselling sessions combined with supervised exercise, with the option of an additional supervised exercise session during alternate weeks Intensity: individual choice

Time: individual choice

Type: individual choice

Volume/progression: increase recreational aerobic physical activity by at least 10 MET-hours/week from baseline in the first 6 months and sustain this change for 3 years

Physical activity interventions for disease-related physical and mental health during and following treatment in people with non- 
Length of intervention: 3 years altogether (three phases- phase 1 adoption, 2 consolidation and 3 maintenance)

Control group: health education material (HEM) intervention receive general health education materials promoting physical activity and healthy nutrition as well as standard surveillance follow-up

Setting: not reported

Supervised or self-directed: supervised or telephone support

Co-interventions: HEM

1. Disease-free survival
2. Overall survival
3. Patient reported outcomes (using SF-36, FACIT-F, PSQI, and HADS questionnaires)
4. Physical fitness (i.e. body mass index, hip and waist circumference, cardiovascular fitness, and physi-
cal function)
5. Physical activity levels (TPAQ)
6. Safety profile according to NCI CTCAE version 3.0
7. Correlative biological markers including biochemical and molecular markers associated with in-
sulin-related growth factor and cytokines associated with the mechanisms of fatigue
8. Economic evaluations including cost-effective analysis and cost utility analysis
9. Predictors of physical activity adherence as assessed by Social Cognitive Determinants of Exercise
questionnaire
Time points measured: baseline and 1 year
Time points reported: baseline and 1 year
Adverse events: not reported
Funding sources: Canada Research Chairs Program Alberta Innovates-Health Solutions,
Alberta Cancer Foundation's Weekend to End Women's Cancers Breast Cancer Chair and
Canadian Cancer Society Research Institute National Health and Medical Research Council (Australia)

\section{Risk of bias}

\begin{tabular}{|c|c|c|}
\hline Bias & Authors' judgement & Support for judgement \\
\hline $\begin{array}{l}\text { Random sequence genera- } \\
\text { tion (selection bias) }\end{array}$ & Unclear risk & Not described \\
\hline $\begin{array}{l}\text { Allocation concealment } \\
\text { (selection bias) }\end{array}$ & Unclear risk & Not described \\
\hline $\begin{array}{l}\text { Blinding of participants } \\
\text { and personnel (perfor- } \\
\text { mance bias) } \\
\text { All outcomes }\end{array}$ & High risk & $\begin{array}{l}\text { This is an open-label study. However, it is not possible to blind participants } \\
\text { and personnel due to the nature of the intervention }\end{array}$ \\
\hline $\begin{array}{l}\text { Blinding of outcome as- } \\
\text { sessment (detection bias) } \\
\text { All outcomes }\end{array}$ & Unclear risk & $\begin{array}{l}\text { Insufficient information available to permit a judgement of 'low risk' or 'high } \\
\text { risk' }\end{array}$ \\
\hline $\begin{array}{l}\text { Incomplete outcome data } \\
\text { (attrition bias) } \\
\text { All outcomes }\end{array}$ & Low risk & Missing data balanced across intervention groups with reasons. $22 \%$ attrition \\
\hline $\begin{array}{l}\text { Selective reporting (re- } \\
\text { porting bias) }\end{array}$ & Low risk & There appears to be no selective reporting of outcomes \\
\hline
\end{tabular}


Courneya 2016 (Continued)

Other bias Low risk The study appears to be free of other problems that could put it at a high risk of bias

Cramer 2016

\section{Study characteristics}

Study design: RCT
Study location: Germany
Inclusion criteria:
1. Colorectal cancer
2. UICC stage I-III
3. Between 2-48 months postsurgery
4. aged at least 18
5. physical and cognitive ability to follow the yoga intervention
Exclusion criteria:
1. UICC stage IV
2. further active oncological diseases
3. diagnosed and pharmacologically
4. treated psychiatric disorder except for cancer-related depression or adjustment disorder
pregnancy or breastfeeding

Participants

Number randomised: 54; 27 intervention, 27 usual care control

Baseline imbalances: more patients in the yoga group treated with colostomy

Withdrawals and exclusions: 6 in the intervention group and 5 from the control group dropped out

Age (SD) (age range):

Total $68.26(9.69)$ years $(40-87)$

control: 67.81 (10.37) years (40-84)

intervention: $68.70(9.13)$ years (49-87)

Gender, n (\%):

Total; Male, 33 (61.1\%) Female, 21 (38.9\%)

Control; Male, 16 (59.3\%) Female, 11 (40.7\%)

Intervention; Male, 17 (63.0\%) Female, 10 (37.0\%)

Ethnicity: not reported

Comorbidities: not reported

SES, n (\%): university degree 16 (29.6\%), employed full time 7 (13\%), retired 35 (64.8\%)

Cancer type: colorectal; colon 24 (44.4\%); rectum 29 (53.7\%)

Cancer stage:

Stage I $=20(37.0 \%)$

Stage II = $11(20.4 \%)$

Stage III = $21(38.9 \%)$

Type of treatment, mean (SD):

Physical activity interventions for disease-related physical and mental health during and following treatment in people with non- 
Cramer 2016 (Continued)

Time since surgery 22.76 (13.09) months (range 3-46 months)

current chemotherapy $\mathrm{n}(\%)$

yes $2(3.7 \%)$

No $52(96.3 \%)$

Receiving or finished treatment:

prior chemotherapy $\mathrm{n}(\%)$

yes $25(46.3 \%)$

No 29 (53.7\%)

prior radiotherapy

yes $12(22.2 \%)$

no $42(77.8 \%)$

Time beyond treatment: 3- 46 months postsurgery 3.7\% still receiving chemotherapy

Time since diagnosis: not reported

Physical activity description
Frequency: 1 weekly class
Intensity: not reported
Time: 90 minutes
Type: Hatha yoga
Progression: postures in each class built up on the previous ones, and difficulty and intensity levels in-
creased during
the course of the programme patients were encouraged to practice at home although no minimum
practice time was required
Length of intervention: 10 weeks of Hatha yoga

Control group: Waiting list control (usual care)

Setting: on-site

Supervised or self-directed: supervised

Co-interventions: none

Outcomes

1. Health-related quality of life (FACT-C)

2. Fatigue (FACIT-F)

3. Anxiety, depression (HADS)

4. Sleep quality (PSQI)

5. Safety (number and severity of adverse events)

6. Spiritual well-being (Functional Assessment of Chronic Illness Therapy - Spirituality)

7. Body awareness/bodily dissociation (Scale of Body Connection (SBC))

8. Body efficacy expectation (Body Efficacy-Expectation Scale)

9. Qualitative Interviews

Time points measured: baseline, week 10, week 22

Time points reported: baseline week 10, week 22

Adverse events: all adverse events occurring during the study period were recorded

Notes $\quad$ Funding source: no external funding

\section{Risk of bias}


Cramer 2016 (Continued)

Random sequence genera- Low risk Block randomisation using the random allocation software was used tion (selection bias)

Allocation concealment $\quad$ Low risk Sealed, sequentially numbered opaque envelopes were used
(selection bias)

(selection bias)

\begin{tabular}{|c|c|c|}
\hline $\begin{array}{l}\text { Blinding of participants } \\
\text { and personnel (perfor- }\end{array}$ & High risk & $\begin{array}{l}\text { This was an open-label RCT. It is not possible however to blind participants } \\
\text { and personnel due to the nature of the intervention }\end{array}$ \\
\hline
\end{tabular}

mance bias)

All outcomes

\begin{tabular}{|c|c|c|}
\hline $\begin{array}{l}\text { Blinding of outcome as- } \\
\text { sessment (detection bias) }\end{array}$ & Unclear risk & $\begin{array}{l}\text { Insufficient information available to permit a judgement of 'low risk' or 'high } \\
\text { risk' }\end{array}$ \\
\hline
\end{tabular}

All outcomes

$\begin{array}{ll}\begin{array}{l}\text { Incomplete outcome data } \\ \text { (attrition bias) }\end{array} & \text { Low risk } \\ \text { All outcomes } & \begin{array}{l}\text { Attrition (20\%) and exclusions were reported with reasons. ITT analysis con- } \\ \text { ducted }\end{array}\end{array}$

All outcomes

\begin{tabular}{lll}
\hline $\begin{array}{l}\text { Selective reporting (re- } \\
\text { porting bias) }\end{array}$ & Low risk & $\begin{array}{l}\text { Clinical trial registry outcomes are consistent with those reported in publica- } \\
\text { tion }\end{array}$ \\
\hline Other bias & Low risk & $\begin{array}{l}\text { More patients in yoga group treated with colostomy, however described ap- } \\
\text { propriate allocation concealment }\end{array}$ \\
\hline
\end{tabular}

Hubbard 2016

\section{Study characteristics}

Study design: RCT
Study location: UK
Inclusion criteria:
1. $\geq 18$ years
2. diagnosed with primary CRC
3. in recovery period following CRC surgery
Exclusion criteria:
1. advanced disease
2. failure of clinical/risk assessment for cardiac rehabilitation
3. deemed unsafe to participate in exercise by cancer nurses during screening
4. severe cognitive impairment
5. unable to communicate in English (no funds available in study for translation services)

Participants

Number randomised: 41; 21 intervention, 20 usual care

Baseline imbalances: Seven (33.3\%) participants allocated to intervention group and four (20\%) allocated to the usual care group were classified as T3. More participants allocated to the intervention group had laparoscopic surgery $(n=6)(28.8 \%)$ versus $n=3(15 \%))$. Four $(19 \%)$ participants allocated to the intervention group had a temporary stoma or permanent stoma, versus nine $(45 \%)$ in usual care (no rehabilitation)

Withdrawals and exclusions:

Follow-up 1; missing 10 participants questionnaire data, and 18 participants accelerometer data

Physical activity interventions for disease-related physical and mental health during and following treatment in people with non- 
Age, mean (SD):

66 (11.31) years

Control, $64.2(11.10)$ years

Intervention, 67.9 (11.49) years

Gender, n (\%):

Male, 27 (65.9\%)

Female, $14(34.1 \%)$

Ethnicity: not reported

SES: not reported

Comorbidities: not reported

Cancer type: colorectal

Colorectal cancer stage: T 0-4, N 0-1, M0

Type of treatment, n, (\%):

Colon surgery, yes 25 (61\%), no $16(39 \%)$

Rectal surgery, yes 16 (39\%), no $25(61 \%)$

Laparoscopic surgery, yes 9 (22\%), no 18 (43.9\%)

Chemotherapy, missing $7(17.1 \%)$, no $27(65.9 \%)$, yes $7(17.1 \%)$

Radiotherapy, missing 7 (17.1\%), no 29 (70.7\%), yes 5 (12.2\%)

Other treatment, missing 3 (7.3\%), no 35 (85.4\%), yes $3(7.3 \%)$

Receiving or finished treatment: both

Time beyond treatment: postsurgery (in recovery period), exact time not reported

Time since diagnosis: not reported

\section{Physical activity description}

Frequency: site $1 \& 2$ once weekly, site 3 twice weekly exercise

Intensity: not reported

Time: $60-90$ min educational sessions

Type: cardiac rehabilitation exercise classes of aerobic and strength training

Volume: not reported

Progression: not reported

Length of programme: varied depending on site (site one: 1 hour class over 10 weeks, site two 12 weeks, site 3 twice weekly over 6 weeks)

Control group: usual care patients were given a booklet by Bowel Cancer UK (a cancer charity) - Staying healthy after bowel cancer

Setting: not reported

Supervised or self-directed: supervised

Co-interventions: weekly education sessions (e.g. diet, physical activity, relaxation/ stress management and cardiac-specific sessions) 
Hubbard 2016 (Continued)

3. Quality of life (EQ5D, FACT-C)

4. Anxiety and depression (HADS)

5. Fatigue (FACIT-Fatigue)

6. Healthcare resource (seven-item self-report questionnaire developed by the research team for the purposes for the study)

7. Physical activity self-efficacy (12-item questionnaire developed by investigators of the Act Well trial

8. Risk perception (operationalisation of the concept in the context of behaviour change research)

Time points measured: baseline, weeks 6, 10, 12 (depending on site), second follow-up assessment was approximately 3 months after the participant had finished cardiac rehabilitation

Time points reported: baseline, up to 12 weeks, 3 months after completion of Cardiac rehabilitation

Adverse events: no adverse events occurred

Notes

Funding source: National Institute for Health Research-Health Service and Development Research

\section{Risk of bias}

\begin{tabular}{|c|c|c|}
\hline Bias & Authors' judgement & Support for judgement \\
\hline $\begin{array}{l}\text { Random sequence genera- } \\
\text { tion (selection bias) }\end{array}$ & Low risk & An automated online randomisation system was used \\
\hline $\begin{array}{l}\text { Allocation concealment } \\
\text { (selection bias) }\end{array}$ & Unclear risk & Not described \\
\hline $\begin{array}{l}\text { Blinding of participants } \\
\text { and personnel (perfor- } \\
\text { mance bias) } \\
\text { All outcomes }\end{array}$ & High risk & $\begin{array}{l}\text { Not possible to blind participants and personnel due to the nature of the inter- } \\
\text { vention }\end{array}$ \\
\hline $\begin{array}{l}\text { Blinding of outcome as- } \\
\text { sessment (detection bias) } \\
\text { All outcomes }\end{array}$ & Unclear risk & $\begin{array}{l}\text { Insufficient information available to permit a judgement of 'low risk' or 'high } \\
\text { risk }\end{array}$ \\
\hline $\begin{array}{l}\text { Incomplete outcome data } \\
\text { (attrition bias) } \\
\text { All outcomes }\end{array}$ & High risk & $\begin{array}{l}\text { Due to missing data at follow-up time points. Time point } 1 \text { : attrition } 24 \% \text { and } \\
43 \% \text { for subjective and objective measures respectively. Time point } 2: \text { attrition } \\
39 \% \text { and } 65 \% \text { for subjective and objective measures, respectively. }\end{array}$ \\
\hline $\begin{array}{l}\text { Selective reporting (re- } \\
\text { porting bias) }\end{array}$ & High risk & Some outcomes described in methods section have not been reported \\
\hline Other bias & High risk & Due to baseline imbalances alongside unclear allocation concealment \\
\hline
\end{tabular}

Kim 2018

\section{Study characteristics}

Methods

Study design: RCT
Study location: Korea

Inclusion criteria:

1. between 18 and 75 years of age

2. confirmed stage II to III CRC

3. completed surgery, radiotherapy, and/or chemotherapy within four weeks to two years prior to study enrolment

Physical activity interventions for disease-related physical and mental health during and following treatment in people with non- 
Kim 2018 (Continued)

4. ECOG performance status of 0 or 1

5. ability to understand and provide written informed consent

\section{Exclusion criteria:}

1. metastatic disease

2. cardiac illness

3. any condition that would make them unsuitable for the study

Participants

Number randomised: 71 (37 intervention, 34 usual care)

Baseline imbalances: no significant difference at baseline

Withdrawals and exclusions: 30 and 28 participants completed study in the intervention and control group, respectively

Age, mean (SD):

control 56.8 (10.2)

intervention $55.7(8.7)$

Gender n (\%):

male control $17(50 \%)$

male intervention $18(48.6 \%)$

Ethnicity: not reported

Comorbidities: not reported

SES (\%):

Income > USD 3000/month control (38.2\%) intervention (54.1\%)

Married control (79.5\%) intervention (75.7\%)

Completed university control (32.4\%) intervention (51.4\%)

Cancer type, $\mathrm{n}(\%)$ :

colon control (55.9\%) intervention (73\%)

rectal control (44.1\%) intervention (27\%)

Colorectal cancer stage:

stage II-III;

stage II control (35.3\%) intervention (56.8\%)

stage III control (64.7\%) intervention (43.2\%)

Type of treatment, $\mathrm{n}(\%)$ :

chemotherapy 98 (80.3\%)

chemo+radiotherapy 13 (10.7\%)

Receiving or finished treatment: finished

Time beyond treatment, mean (SD):

control 10.6 months (8.4) intervention 10.8 months (5.8)

Time since diagnosis: not reported 
Kim 2018 (Continued)

Frequency: not reported

Intensity: moderate and vigorous intensity

Time: not reported

Type: aerobic and resistance home-based exercise e.g. brisk walking,

hiking and stationary bike riding were recommended for

aerobic exercise 30 min resistance exercise (12-15 reps) DVD provided

Volume: weeks 1-6 $18 \mathrm{MET} /$ hours per week

progression: after week 6 increase to 27 MET hours per week

Length of programme: 12 weeks

Control group: usual care

Setting: home-based with four meetings with exercise trainer, telephone calls and small group sessions

Supervised or self-directed: self-directed, physical activity logs checked by exercise specialists

Co-interventions: none

1. Quality of life
Secondary outcomes:
2. Fatigue
3. Levels of physical activity
4. Anthropometric measurements
5. Levels of physical activity
Time points measured: baseline and 12 weeks
Time points reported: baseline and 12 weeks
Adverse events: not reported

Notes Funding source: not described

\section{Risk of bias}

\begin{tabular}{lll}
\hline Bias & Authors' judgement & Support for judgement \\
\hline $\begin{array}{l}\text { Random sequence genera- } \\
\text { tion (selection bias) }\end{array}$ & Low risk & A computer-generated random number sequence was used \\
\hline $\begin{array}{l}\text { Allocation concealment } \\
\text { (selection bias) }\end{array}$ & Unclear risk & Not described \\
\hline $\begin{array}{l}\text { Blinding of participants } \\
\begin{array}{l}\text { and personnel (perfor- } \\
\text { mance bias) } \\
\text { All outcomes }\end{array}\end{array}$ & High risk & $\begin{array}{l}\text { Not possible to blind participants and personnel due to the nature of the inter- } \\
\text { vention }\end{array}$ \\
\hline
\end{tabular}

\begin{tabular}{ll}
\hline Blinding of outcome as- & Unclear risk
\end{tabular}

\begin{tabular}{|c|c|c|}
\hline $\begin{array}{l}\text { Incomplete outcome data } \\
\text { (attrition bias) } \\
\text { All outcomes }\end{array}$ & Low risk & $\begin{array}{l}\text { Attrition } 18.9 \% \text { in intervention and } 17.6 \% \text { in control group. Exclusions report- } \\
\text { ed with reasons. ITT analysis conducted }\end{array}$ \\
\hline
\end{tabular}

$\begin{aligned} & \text { Selective reporting (re- } \\ & \text { porting bias) }\end{aligned} \quad$ Low risk There appears to be no selective reporting of outcomes


Kim 2018 (Continued)

Other bias Low risk The study appears to be free of other problems that could put it at a high risk of bias

\section{Study characteristics}

Study design: RCT
Study location: South Korea
Inclusion criteria:
1. resident of Seoul or Gyeonggi-do
2. between 18 and 75 years of age
3. confirmed stage II to III CRC
4. completed surgery, radiotherapy, and/or chemotherapy within four weeks to two years prior to study
enrolment
5. ECOG performance status of 0 or 1
6. ability to understand and provide written informed consent
7. not planning extended absences in the three months subsequent to enrolment
Exclusion criteria:
1. evidence of recurrent or metastatic disease
2. participation in regular structured physical activity at moderate-intensity exceeding 200 min/week
3. pregnant or planning to be pregnant within six months
4. we additionally excluded ostomy patients due to lack of evidence in safety of strength training

Participants

Number randomised: 123; (62 intervention; 61 usual care)

Baseline imbalances: no significant difference at baseline

Withdrawals and exclusions: 13 lost to follow-up in control group; 11 lost to follow-up in intervention group

Age, mean (SD):

Total $56.3(9.7)$

control 56.3 (9.7)

intervention $56.3(9.9)$

Gender n (\%):

male $59(48 \%)$

male control 28 (45.9\%)

male intervention 31 (50\%)

Ethnicity: not reported

Comorbidities: not reported

SES (\%):

Income > USD 3000/month (44.7\%)

Married (81\%)

Completed university $(48.8 \%)$

Physical activity interventions for disease-related physical and mental health during and following treatment in people with non- 
Lee 2017 (Continued)

\author{
Cancer type, n (\%): \\ colon $84(68.9 \%)$ \\ rectal $38(31.1 \%)$ \\ Colorectal cancer stage: \\ stage II-III; \\ stage II $(46.7 \%)$ \\ stage III $(53.3 \%)$ \\ Type of treatment, $\mathrm{n}(\%)$ : \\ chemotherapy 98 (80.3\%) \\ chemo+radiotherapy $13(10.7 \%)$ \\ Receiving or finished treatment: finished \\ Time beyond treatment, mean (SD): \\ 9.8 (6.3) months
}

Time since diagnosis: not reported

\title{
Physical activity description:
}

Frequency: encouraged to do 10,000 steps daily and daily resistance exercise programme Intensity: of these 10,000 steps, participants were asked to complete 3000 steps as exercise that increased their heart rate up to $65 \%$ of their age-predicted maximum heart rate

Time: not reported

Type: aerobic and resistance home-based exercise e.g. brisk walking, hiking and stationary bike riding were recommended for aerobic exercise 30 min resistance exercise using their own body weight that could be performed daily at home Volume: weeks 1-6 $18 \mathrm{MET} /$ hours per week progression: after week 6 increase to 27 MET hours per week Length of programme: 12 weeks

Control group: usual care

Setting: home based with three sessions in hospital based clinic

Supervised or self-directed: self-directed, physical activity logs checked by exercise specialists

Co-interventions: none

Outcomes
Secondary outcomes:
2. Biomarkers
3. Physical fitness
4. Body composition
5. Levels of physical activity
Time points measured: baseline and 12 weeks
Time points reported: baseline and 12 weeks
Adverse events: not reported
Funding source: National R\&D programme for cancer control and the national research foundation of
Korea


Lee 2017 (Continued)

\section{Risk of bias}

\begin{tabular}{|c|c|c|}
\hline Bias & Authors' judgement & Support for judgement \\
\hline $\begin{array}{l}\text { Random sequence genera- } \\
\text { tion (selection bias) }\end{array}$ & Low risk & Computer-generated random number sequence was used \\
\hline $\begin{array}{l}\text { Allocation concealment } \\
\text { (selection bias) }\end{array}$ & Unclear risk & Not described \\
\hline $\begin{array}{l}\text { Blinding of participants } \\
\text { and personnel (perfor- } \\
\text { mance bias) } \\
\text { All outcomes }\end{array}$ & High risk & $\begin{array}{l}\text { Not possible to blind participants and personnel due to the nature of the inter- } \\
\text { vention }\end{array}$ \\
\hline $\begin{array}{l}\text { Blinding of outcome as- } \\
\text { sessment (detection bias) } \\
\text { All outcomes }\end{array}$ & Unclear risk & Not described \\
\hline $\begin{array}{l}\text { Incomplete outcome data } \\
\text { (attrition bias) } \\
\text { All outcomes }\end{array}$ & Low risk & $\begin{array}{l}\text { Attrition (19\%) and exclusions were reported with reasons. ITT analysis con- } \\
\text { ducted }\end{array}$ \\
\hline $\begin{array}{l}\text { Selective reporting (re- } \\
\text { porting bias) }\end{array}$ & Low risk & There appears to be no selective reporting of outcomes \\
\hline Other bias & Low risk & $\begin{array}{l}\text { The study appears to be free of other problems that could put it at a high risk } \\
\text { of bias }\end{array}$ \\
\hline
\end{tabular}

Lewis 2016

\section{Study characteristics}

Study design: RCT
Study location: UK
Inclusion criteria:
1. a histologically confirmed diagnosis of colorectal cancer with Dukes stages A-C
2. completed cancer treatment within the last 24 months (later increased to 3 years)
3. be able to understand spoken and written English score of 80 or more on the Karnofsky Performance
Status Scale
Exclusion criteria:
1. already meeting general physical activity guidelines of 150 min of moderate physical activity or 75
min of vigorous intensity physical activity per week
2. recent myocardial infarction
3. uncontrolled hypertension
4. pacemaker
5. unstable angina

Number randomised: $28 ; 14$ control; 14 intervention
Baseline imbalances: no significant difference between groups at baseline
Withdrawals and exclusions:

Physical activity interventions for disease-related physical and mental health during and following treatment in people with non- 
Control; 3 dropouts (two at three months, one at six months)

Intervention: 5 dropouts (two at three months, three at six months)

Age, mean (SD):

Total mean age; 64.75

Control $65.5(9.2)$

Intervention 64.0 (14.8)

Gender:

Control: 7 male, 7 female

Intervention: 8 male, 6 female

Ethnicity: not reported

Comorbidities: not reported

SES: not reported

Cancer type: colorectal

Colorectal cancer stage: Dukes A-C

Type of treatment, $\mathrm{n}(\%)$ :

Surgery; 28 (100\%)

Chemotherapy; 10 control (71\%), 2 intervention (14\%)

Receiving or finished treatment: finished treatment

Time beyond treatment: within three years of completion of treatment

Time since diagnosis, months: control 13, intervention 12 months

\section{Physical activity description}

Frequency: supervised exercise sessions took place twice per week for the first four weeks. This was tapered off to once per week for the second four weeks. During the last month of the intervention participants continued with the exercise at home Intensity: moderate and vigorous

Time: not reported

Type: not reported

Volume: encouraged to achieve 150 min of moderate to vigorous physical activity per week

Progression: not reported

Length of programme: supervised exercises for three months

Control group: encouraged to continue with their usual lifestyle habits. Supervised exercise sessions were offered after their last appointment

Setting: home and supervised

Supervised or self-directed: both

Co-interventions: none version and accelerometry)

2. Behavioural regulation (Behavioural Regulation in Exercise Questionnaire version 2)

Physical activity interventions for disease-related physical and mental health during and following treatment in people with non- 
Lewis 2016 (Continued)

3. Psychological needs satisfaction (psychological needs satisfaction in exercise scale)

4. QoL (FACT-G, FACT-C)

5. Fatigue (FACT-F)

6. Intention to exercise (Intention to exercise scale)

7. Barriers to exercise (Barriers to Exercise scale)

8. Physical fitness (treadmill test)

9. Upper body strength (grip strength, arm-curl test, 30-Second Chair Stand Test)

10. Body composition (Bioelectrical impedence, waist and waist to hip ratio, height weight, BMI)

Time points measured: baseline, 3 and 6 months

Time points reported: baseline, 3 and 6 months

Adverse events: exercise was deemed safe; there were no adverse events throughout the intervention

Notes Funding source: University of East Anglia

\section{Risk of bias}

\begin{tabular}{lll}
\hline Bias & Authors' judgement & Support for judgement \\
\hline $\begin{array}{l}\text { Random sequence genera- } \\
\text { tion (selection bias) }\end{array}$ & Low risk & Participants randomised using computer generated list (nQuery software) \\
\hline $\begin{array}{l}\text { Allocation concealment } \\
\text { (selection bias) }\end{array}$ & Low risk & $\begin{array}{l}\text { A person independent of the research team kept the randomisation sequence. } \\
\text { After the completion of the baseline assessments, the research team phoned } \\
\text { the independent person to obtain group allocation. }\end{array}$ \\
\hline
\end{tabular}

Not possible to blind participants and personnel due to the nature of the inter-

and personnel (perfor- vention

mance bias)

All outcomes

$\begin{array}{lll}\text { Blinding of outcome as- } & \text { High risk } & \begin{array}{l}\text { Data collection and analyses were carried out by a researcher not blind to } \\ \text { group allocation }\end{array}\end{array}$

All outcomes

\begin{tabular}{lll}
\hline $\begin{array}{l}\text { Incomplete outcome data } \\
\text { (attrition bias) } \\
\text { All outcomes }\end{array}$ & Low risk & $\begin{array}{l}\text { Attrition (28\%) and exclusions were reported with reasons. ITT analysis con- } \\
\text { ducted }\end{array}$ \\
\hline $\begin{array}{l}\text { Selective reporting (re- } \\
\text { porting bias) }\end{array}$ & Low risk & $\begin{array}{l}\text { Clinical trial registry outcomes are consistent with those reported in publica- } \\
\text { tion }\end{array}$ \\
\hline Other bias & Low risk & $\begin{array}{l}\text { The study appears to be free of other problems that could put it at a high risk } \\
\text { of bias. No significant difference between groups at baseline. }\end{array}$ \\
\hline
\end{tabular}

\section{McDermott 2017}

\section{Study characteristics}

$\begin{array}{ll}\text { Methods } & \text { Study design: RCT } \\ & \text { Study location: Northern Ireland }\end{array}$

\section{Inclusion criteria:}

Physical activity interventions for disease-related physical and mental health during and following treatment in people with non- 
1. Dukes A-C colorectal cancer patients at least 6 weeks post any type anticancer treatment, as identified by the colorectal oncologic and surgical teams.

2. males and females over 18 years of age

3. physically able to undertake the intervention

4. ambulatory and without use of a walking aid

\section{Exclusion criteria:}

1. still undergoing and/or scheduled for further anti-cancer treatment

2. any presence of cognitive impairment

3. pregnant or possibility of being pregnant

4. known comorbidities which would severely impact upon physical functioning or nutritional status

5. already meeting the current recommended physical activity guidelines

6. unable to understand and communicate in written and oral English

Participants

Number randomised: 23; 11 control; 12 intervention

Baseline imbalances: mean weight and hip circumference, FACT-C and FACIT-F results were significantly higher in the intervention group. Fatigue scores were significantly lower in the intervention group

Withdrawals and exclusions: 3 dropouts before week 12 (2 control; 1 intervention), 1 further dropout in intervention group at week 24

Age, mean (SD): total 63 (9) years; control 62.6 (9.1) intervention 63.6 (9.5)

Gender n (\%):

Male; 6 (54.5\%) control; 10 (83.3\%) intervention

Female; 5 (45.5\%) control; 2 (16.7\%) intervention

Ethnicity: not reported

Comorbidities: not reported

SES (employment):

Professional: 5 (45.5\%) control; 5 (41.6\%) intervention

Managerial: 0 control; 3 (25\%) intervention

Clerical; 1 (9\%) control; 2 (16.7\%) intervention

Manual; 5 (45.5\%) control; 2 (16.7\%) intervention

Cancer type: colorectal

Colon; 9 (81.8\%) control; 8 (66.7\%) intervention

Rectal; 2 (18.2\%) control; 4 (33.3\%) intervention

Colorectal cancer stage:

1a: 0 control; 1 (8.3\%) intervention

2a/2b: 6 (54.5\%) control; 7 (58.3\%) intervention

3a/3b/3c: 5 (45.5\%) control; 4 (33.3\%) intervention

Type of treatment:

Surgery only: 1 (18.2\%) control; 4 (33.3\%) intervention

Surgery and chemotherapy; 8 (72.7\%) control; 6 (50\%) intervention

Radio/chemo and surgery: 1 (9.1\%) control; 2 (16.7\%) intervention

Receiving or finished treatment: finished (at least 6 weeks post-treatment) 
McDermott 2017 (Continued)

Time beyond treatment: an average of 24 months post-treatment (25 (17) months in control group; 23

(19) months in the intervention group)

Time since diagnosis: not reported

Physical activity description:
Frequency: not reported
Intensity: moderate-intensity
Time: not reported
Type: walking and strengthening intervention
Volume: not reported
Progression: aim to reach at least 150 minutes a week of moderate-intensity aerobic activity and
strengthening goal of 3 sets of $8-15$ repetitions, 2 -3 days a week
Length of programme: 12 weeks
Control group: usual care; participants will receive the intervention information on their last visit
Setting: home based
Supervised or self-directed: self-directed with weekly telephone calls
Co-interventions: none

1. Feasibility
2. Levels of physical activity (accelerometer)
3. Cardiovascular endurance (6-minute walk test)
4. Lower limb strength (timed sit to stand)
5. Quality of life (FACT-C, EQ5D-3L, PANAS)
6. Demographics
7. Anthropometric tests (BMI, height, weight, hip and waist measurements)
8. Biological markers (venous and capillary blood samples)
9. Fatigue (MFSI-SF)
Time points measured: baseline 12, 24 weeks
Time points reported: baseline 12, 24 weeks
Adverse events: not reported

Notes Funding source: University of Ulster

\section{Risk of bias}

\begin{tabular}{|c|c|c|}
\hline Bias & Authors' judgement & Support for judgement \\
\hline $\begin{array}{l}\text { Random sequence genera- } \\
\text { tion (selection bias) }\end{array}$ & Low risk & A random numbers table was used \\
\hline $\begin{array}{l}\text { Allocation concealment } \\
\text { (selection bias) }\end{array}$ & Low risk & Numbered concealed envelopes were used \\
\hline $\begin{array}{l}\text { Blinding of participants } \\
\text { and personnel (perfor- } \\
\text { mance bias) } \\
\text { All outcomes }\end{array}$ & High risk & $\begin{array}{l}\text { Not possible to blind participants and personnel due to the nature of the inter- } \\
\text { vention }\end{array}$ \\
\hline $\begin{array}{l}\text { Blinding of outcome as- } \\
\text { sessment (detection bias) } \\
\text { All outcomes }\end{array}$ & High risk & $\begin{array}{l}\text { Researcher carrying out the outcome measures was aware of the participants } \\
\text { group allocation }\end{array}$ \\
\hline
\end{tabular}


McDermott 2017 (Continued)

Incomplete outcome data Low risk Attrition (17\%) and exclusions were reported with reasons. ITT analysis con(attrition bias) ducted

All outcomes

\begin{tabular}{lll}
$\begin{array}{l}\text { Selective reporting (re- } \\
\text { porting bias) }\end{array}$ & Low risk & $\begin{array}{l}\text { Outcomes reported are consistent with outcomes to be reported in clinical tri- } \\
\text { al register }\end{array}$ \\
\hline Other bias & Low risk & $\begin{array}{l}\text { Baseline imbalances present, however described appropriate allocation con- } \\
\text { cealment }\end{array}$
\end{tabular}

Nuri 2016

\section{Study characteristics}

\begin{tabular}{|c|c|}
\hline Methods & $\begin{array}{l}\text { Study design: RCT } \\
\text { Study location: Iran } \\
\text { Inclusion criteria: not reported } \\
\text { Exclusion criteria: not reported }\end{array}$ \\
\hline \multirow[t]{14}{*}{ Participants } & Number randomised: $30 ; 15$ control, 15 intervention \\
\hline & Baseline imbalances: no significant differences at baseline \\
\hline & $\begin{array}{l}\text { Withdrawals and exclusions: three subjects in control group who did not participate in the final exami- } \\
\text { nations were excluded }\end{array}$ \\
\hline & Age, mean (SD): $51.56(11.28)$ years \\
\hline & Gender: 30 (100\%) males \\
\hline & Ethnicity: not reported \\
\hline & Comorbidities: not reported \\
\hline & SES: not reported \\
\hline & Cancer type: colorectal \\
\hline & Colorectal cancer stage: stage I-III, confirmed via email from author \\
\hline & Type of treatment: surgery (no other treatment reported) \\
\hline & Receiving or finished treatment: not reported \\
\hline & Time beyond treatment: between 2 and 3 months postsurgery \\
\hline & Time since diagnosis: not reported \\
\hline
\end{tabular}

Interventions

Physical activity description:

Frequency: 3 times per week

Intensity: $50 \%$ to $60 \%$ of target heart rate

Time: 45 minute (including 5 min warm up cool down and 5 minute rest)

Type: aerobic/walking

Volume: not reported

Progression:

Length of programme: 8 weeks

Physical activity interventions for disease-related physical and mental health during and following treatment in people with non- 
Nuri 2016 (Continued)
Control group: usual care
Setting: not reported
Supervised or self-directed: not reported
Co-interventions: participants completed the 3 -day diet recall forms and were instructed to maintain normal dietary habits throughout the study

\begin{tabular}{ll}
\hline Outcomes & 1. Leptin and ghrelin \\
2. Anthropometric and body composition measures (BMI, waist circumference, waist, hip ratio, fat \\
mass, lean mass) \\
3. Metabolic characteristics (glucose, insulin, HOMA-IR) \\
4. Composition of subjects' diets \\
5. Physical fitness: Rockport one mile fitness walking \\
Time points measured: baseline, 8 weeks and 9 weeks (i.e. after 1 week of detraining) \\
Time points reported: baseline, 8 weeks and 9 weeks (i.e. after 1 week of detraining) \\
Adverse events: not reported \\
Notes
\end{tabular}

\section{Risk of bias}

\begin{tabular}{|c|c|c|}
\hline Bias & Authors' judgement & Support for judgement \\
\hline $\begin{array}{l}\text { Random sequence genera- } \\
\text { tion (selection bias) }\end{array}$ & Unclear risk & Method of randomisation not reported \\
\hline $\begin{array}{l}\text { Allocation concealment } \\
\text { (selection bias) }\end{array}$ & Unclear risk & Not described \\
\hline $\begin{array}{l}\text { Blinding of participants } \\
\text { and personnel (perfor- } \\
\text { mance bias) } \\
\text { All outcomes }\end{array}$ & High risk & $\begin{array}{l}\text { Insufficient information available to permit a judgement but probably 'high } \\
\text { risk' }\end{array}$ \\
\hline $\begin{array}{l}\text { Blinding of outcome as- } \\
\text { sessment (detection bias) } \\
\text { All outcomes }\end{array}$ & Unclear risk & $\begin{array}{l}\text { Insufficient information available to permit a judgement of 'low risk' or 'high } \\
\text { risk' }\end{array}$ \\
\hline $\begin{array}{l}\text { Incomplete outcome data } \\
\text { (attrition bias) } \\
\text { All outcomes }\end{array}$ & Low risk & $20 \%$ attrition \\
\hline $\begin{array}{l}\text { Selective reporting (re- } \\
\text { porting bias) }\end{array}$ & High risk & Some outcomes described in methods section have not been reported \\
\hline Other bias & Low risk & $\begin{array}{l}\text { The study appears to be free of other problems that could put it at a high risk } \\
\text { of bias }\end{array}$ \\
\hline
\end{tabular}

Pinto 2013

\section{Study characteristics}

Methods Study design: RCT

Physical activity interventions for disease-related physical and mental health during and following treatment in people with non- 
Pinto 2013 (Continued)

\section{Study location: USA}

\section{Inclusion criteria:}

1. sedentary adults

2. stage 1-3 colorectal cancer

3. finished treatment surgery and/or adjuvant therapy

4. less than 5 years postdiagnosis

5. English speaking

6. access to a telephone

7. CVD or diabetes with consent

\section{Exclusion criteria:}

1. prior history of cancer

2. medical or current psychiatric illness (e.g. orthopaedic problems) that could make compliance with the study protocol difficult or unsafe

Participants

Number randomised: 46; 20 to physical activity group, 26 to control group

Baseline imbalances: more people in employment in the control group

Withdrawals and exclusions: 4 withdrew; 1 in physical activity group, 3 in control group

Age: 57.3 years (SD 9.7); intervention 55.6 (8.24) control 59.5 (11.2)

Gender: male and female (57\%)

Ethnicity: majority white

Comorbidities: not reported

SES: majority attended college and had a median household income of USD 60,000

Cancer type: colorectal cancer; $57 \%$ colon cancer, $43 \%$ rectal cancer

Cancer stage: stage I-III

Type of treatment: $100 \%$ surgery or surgery with adjuvant therapy $20 / 46$ radiotherapy; 38/46 chemotherapy

Receiving or finished treatment: finished

Time beyond treatment: $<5$ years since completion of treatment

Time since diagnosis: mean 2.99 years since diagnosis

Interventions

\section{Physical activity description:}

Frequency: encouraged 10 minutes at least 2 days per week for three weeks, goals were increased over the 12 weeks to 30 minutes a day, 5 days per week

Intensity: moderate $64 \%$ to $76 \%$ estimated heart rate max

Type: e.g. brisk walking, biking, home-based exercise equipment

Length of intervention: 12 weeks

Control group: contact control group (received weekly calls to match frequency of contact with physical activity group and survivorship tip sheets)

Setting: home based

Supervised or self-directed: self-directed

Co-interventions: none

Physical activity interventions for disease-related physical and mental health during and following treatment in people with non- 
Pinto 2013 (Continued)

Outcomes

1. Levels of physical activity (7-day recall, Community Healthy Activities Model Program for Seniors, accelerometry),

2. Movement to action/maintenance stage of motivational

readiness,

3. Submaximal aerobic fitness (Treadwalk test)

4. Psychological outcomes (FACT-C)

5. Fatigue (FACT-F)

6. Anthropometric measurements (height, weight, BMI)

Time points measured: baseline, 3, 6 and 12 months

Time points reported:

Adverse events: not reported

Notes Funding source: National Cancer Institute

\section{Risk of bias}

\begin{tabular}{|c|c|c|}
\hline Bias & Authors' judgement & Support for judgement \\
\hline $\begin{array}{l}\text { Random sequence genera- } \\
\text { tion (selection bias) }\end{array}$ & Unclear risk & Not described \\
\hline $\begin{array}{l}\text { Allocation concealment } \\
\text { (selection bias) }\end{array}$ & Unclear risk & Not described \\
\hline $\begin{array}{l}\text { Blinding of participants } \\
\text { and personnel (perfor- } \\
\text { mance bias) } \\
\text { All outcomes }\end{array}$ & High risk & $\begin{array}{l}\text { Not possible to blind participants and personnel } \\
\text { due to the nature of the intervention }\end{array}$ \\
\hline $\begin{array}{l}\text { Blinding of outcome as- } \\
\text { sessment (detection bias) } \\
\text { All outcomes }\end{array}$ & Low risk & $\begin{array}{l}\text { Assessments conducted by a staff member who was blinded to the partici- } \\
\text { pants' group assignments }\end{array}$ \\
\hline $\begin{array}{l}\text { Incomplete outcome data } \\
\text { (attrition bias) } \\
\text { All outcomes }\end{array}$ & Low risk & Reasons for missing outcome data were described ( $9 \%$ attrition) \\
\hline $\begin{array}{l}\text { Selective reporting (re- } \\
\text { porting bias) }\end{array}$ & Low risk & Outcomes reported are consistent with study protocol \\
\hline Other bias & High risk & Due to baseline imbalances alongside unclear allocation concealment \\
\hline
\end{tabular}

Van Blarigan 2019

\section{Study characteristics}

Study design: RCT
Study location: USA
Inclusion criteria:
1. stage II-III colon or rectal cancer then expanded to include stage I and stage IV
2. previous completion of cancer-directed therapy $\geq 3$ months and < 2years prior to enrolment (time af-
ter completion of treatment was dropped)

Physical activity interventions for disease-related physical and mental health during and following treatment in people with non- 
Van Blarigan 2019 (Continued)

3. disease free status at enrolment

4. ability to reliably access the Internet and a mobile phone and navigate websites

5. ability to speak and read English

\section{Exclusion criteria:}

1. any of 19 specific contraindications to moderate-to-vigorous physical activity

2 . very active at baseline (defined as engaging in exercise for $\geq 30 \mathrm{~min}$ for $\geq 5$ days a week)

\section{Participants}

Number randomised: $42 ; 21$ to intervention group, 21 to control group

Baseline imbalances: control group more active than intervention group

Withdrawals and exclusions: 0 lost to follow-up in intervention group, 2 in control group

Age: 54 years (SD 11); intervention 56 (12); control 54 (11)

Gender: male (41\%) and female (59\%)

Ethnicity: white (73\%), African American/black (2\%), Asian (12\%), other (12\%)

Comorbidities: not reported

SES: college degree (93\%), employed (63\%)

Cancer type: colon (56\%), rectal (44\%)

Cancer stage: stage I (20\%), II (20\%), III (59\%), IV (2\%)

Type of treatment: $100 \%$ surgery or surgery with adjuvant therapy $20 / 46$ radiotherapy; 38/46 chemotherapy

Receiving or finished treatment: finished

Time beyond treatment: not reported

Time since diagnosis: mean 1.5 years (SD 1.5)

\section{Physical activity description}

Frequency: encouraged to build up to 150 mins per week of moderate activity or 75 min per week of vigorous activity Intensity: moderate or vigorous

Type: e.g. brisk walking, biking, jogging, resistance exercise

Length of intervention: 12 weeks

Control group: received printed educational material about exercise after cancer

Setting: home based

Supervised or self-directed: fit bit flex, daily text messages and counselled through print materials

Co-interventions: none

Outcomes

1. Levels of physical activity (Actigraph),

2. Fitbit wear time (\# days with data / \# days of observation),

3. Response to text messages (\# of messages responded to / \# of messages that asked for a response),

Time points measured: baseline and 12 weeks (adverse events measured at baseline 4, 8 and 12 weeks)

Time points reported: baseline, 12 weeks

Physical activity interventions for disease-related physical and mental health during and following treatment in people with non- 
Van Blarigan 2019 (Continued)

Adverse events: reported

\section{Notes}

\section{Risk of bias}

\begin{tabular}{|c|c|c|}
\hline Bias & Authors' judgement & Support for judgement \\
\hline $\begin{array}{l}\text { Random sequence genera- } \\
\text { tion (selection bias) }\end{array}$ & Low risk & Computer-generated randomisation scheme was used \\
\hline $\begin{array}{l}\text { Allocation concealment } \\
\text { (selection bias) }\end{array}$ & Unclear risk & $\begin{array}{l}\text { Whether the treatment assignment was concealed from study personnel and } \\
\text { participants was not described }\end{array}$ \\
\hline $\begin{array}{l}\text { Blinding of participants } \\
\text { and personnel (perfor- } \\
\text { mance bias) } \\
\text { All outcomes }\end{array}$ & High risk & $\begin{array}{l}\text { Not possible to blind participants and personnel due to the nature of the inter- } \\
\text { vention }\end{array}$ \\
\hline $\begin{array}{l}\text { Blinding of outcome as- } \\
\text { sessment (detection bias) } \\
\text { All outcomes }\end{array}$ & High risk & Authors state "non-blinded" trial \\
\hline $\begin{array}{l}\text { Incomplete outcome data } \\
\text { (attrition bias) } \\
\text { All outcomes }\end{array}$ & Low risk & $\begin{array}{l}\text { Low attrition ( } 5 \% \text { in intervention arm and } 10 \% \text { in control arm) with reasons re- } \\
\text { ported }\end{array}$ \\
\hline $\begin{array}{l}\text { Selective reporting (re- } \\
\text { porting bias) }\end{array}$ & High risk & Some outcomes described in methods section have not been reported \\
\hline Other bias & High risk & Control group more active at baseline \\
\hline
\end{tabular}

\section{Van Vulpen 2016}

\section{Study characteristics}

Study design: RCT
Study location: The Netherlands
Inclusion criteria:
1. a histological diagnosis of colon cancer less than 10 wk before study recruitment
2. stage M0
3. scheduled for chemotherapy
4. age 25 to 75 yr
5. able to read and understand the Dutch language
6. Karnofsky Performance Status of 60 or higher
7.able to walk 100 m or more
Exclusion criteria:
1. the presence of contraindications for physical activity (as assessed through the Revised Physical Ac-
tivity Readiness Questionnaire)
2. treatment for cancer in the 5 years preceding recruitment (except for basal skin cancer)

Participants Number randomised: 33; 17 intervention, 16 control 
Van Vulpen 2016 (Continued)

Baseline imbalances: participants in the intervention group were, on average, shorter and heavier

Withdrawals and exclusions: 5 lost to follow-up; 3 in control group, 2 in intervention group

Age, mean (SD):

Total mean age 58.1; intervention 58.1 (10.3); control 58.1 (9.6)

Gender, n (\%):

Male; $11(68.8 \%)$ control; $10(58.8 \%)$ intervention

Female; 5 (31.3\%) control; 7 (41.2\%) intervention

Ethnicity: not reported

Comorbidities:

SES:

High-income; 9 (56.2\%) control; 8 (47.1\%) intervention

Married; 11 (68.8\%) control; 12 (70.6\%) intervention

Education; low; 3 (18.8\%) control; 1 (5.9\%) intervention; medium; 4 (25.0\%) control; 7 (41.2\%) intervention; high; 9 (56.2\%) control; 8 (47.1\%) intervention; unknown 1 (5.9\%) intervention

Cancer type: colon

Colorectal cancer stage: stage M0

Type of treatment: $100 \%$ received surgery, $100 \%$ received chemotherapy, $18.4 \%$ received radiotherapy

Receiving or finished treatment: $81.8 \%$ undergoing chemotherapy

Time beyond treatment: majority receiving treatment

Time since diagnosis: diagnosis of colon cancer less than 10 weeks before study recruitment

\section{Physical activity description:}

Frequency: supervised ( 2 times per week), unsupervised ( 3 times per week) Intensity: based on heart rate at ventilatory threshold and Borg

Time: supervised (1-hour session) unsupervised (30 mins)

Type: supervised - aerobic and muscle strengthening exercise (all large muscle groups)

Volume: not reported

Progression: the muscle strengthening training started with $2 \times 10$ repetitions (65\% 1-repetition maximum (RM) and gradually increased to reach $1 \times 10$ repetitions (75\% 1-RM) and $1 \times 20$ repetitions (45\% 1RM)) by the end of the programme Length of programme: 18 weeks

Control group: were instructed to maintain their usual physical activity pattern.

Setting: not reported

Supervised or self-directed: both

Co-interventions: none

Outcomes
1. Fatigue (Multidimensional Fatigue Inventory (MFI) and the Fatigue Quality List)
2. QoL, anxiety, and depression: (EORTC QLQ-C30), and a general measure of QoL, SF-36 and (HADS)
3. Physical fitness (peak oxygen uptake $\left(\mathrm{VO}_{2}\right.$ peak)-Cardiopulmonary exercise test, anthropometry
(bodyweight and height)
4. Chemotherapy completion rate: weeks
5. Adherence (attendance percentage of the total offered class)
6. Physical activity level (short questionnaire to assess health-enhancing physical activity)
Time points measured: baseline, 18 and 36 weeks 
Van Vulpen 2016 (Continued)

Time points reported: baseline, 18 and 36 weeks

Adverse events: no serious adverse events related to exercise were observed during the study period

Notes $\quad$ Funding source: The Netherlands Organisation for Health Research and Development, the Dutch Cancer Society and the Dutch Pink Ribbon Foundation

\section{Risk of bias}

\begin{tabular}{|c|c|c|}
\hline Bias & Authors' judgement & Support for judgement \\
\hline $\begin{array}{l}\text { Random sequence genera- } \\
\text { tion (selection bias) }\end{array}$ & Low risk & Computer generated randomisation was used \\
\hline $\begin{array}{l}\text { Allocation concealment } \\
\text { (selection bias) }\end{array}$ & Low risk & $\begin{array}{l}\text { Participants were randomised by a concealed computer generated randomi- } \\
\text { sation }\end{array}$ \\
\hline $\begin{array}{l}\text { Blinding of participants } \\
\text { and personnel (perfor- } \\
\text { mance bias) } \\
\text { All outcomes }\end{array}$ & High risk & $\begin{array}{l}\text { Not possible to blind participants and personnel due to the nature of the inter- } \\
\text { vention }\end{array}$ \\
\hline $\begin{array}{l}\text { Blinding of outcome as- } \\
\text { sessment (detection bias) } \\
\text { All outcomes }\end{array}$ & Low risk & $\begin{array}{l}\text { Outcome measures were assessed by researchers not involved with the exer- } \\
\text { cise interventions }\end{array}$ \\
\hline $\begin{array}{l}\text { Incomplete outcome data } \\
\text { (attrition bias) } \\
\text { All outcomes }\end{array}$ & Low risk & $\begin{array}{l}\text { Attrition balanced across groups, attrition (16\%) and exclusions were reported } \\
\text { with reasons. ITT analysis conducted }\end{array}$ \\
\hline $\begin{array}{l}\text { Selective reporting (re- } \\
\text { porting bias) }\end{array}$ & Low risk & Outcomes reported are consistent with those in study protocol \\
\hline Other bias & Low risk & $\begin{array}{l}\text { Baseline imbalances present, however described appropriate allocation con- } \\
\text { cealment }\end{array}$ \\
\hline
\end{tabular}

Waart 2017

\section{Study characteristics}

$\begin{array}{ll}\text { Methods } & \text { Study design: RCT } \\ \text { Study location: The Netherlands } \\ \text { Inclusion criteria: } \\ \text { 1. there was no upper age limit } \\ \text { Exclusion criteria: } \\ \text { 1.serious orthopedic, cardiovascular, or cardiopulmonary conditions } \\ \text { 2. were suffering from malnutrition } \\ \text { 3. had serious psychiatric or cognitive problems } \\ \text { 4. did not have basic fluency in Dutch }\end{array}$

Participants

Number randomised: $23 ; 7$ on-track, 8 onco-move, 8 usual care

Physical activity interventions for disease-related physical and mental health during and following treatment in people with non- 
Waart 2017 (Continued)

Baseline imbalances: participants in onco-move were more frequently male, had lower education and more often received laparoscopic surgery while participants in on-track had less comorbidity. Participants in onco-move tended to have higher physical fitness levels than those in on-track or usual care

Withdrawals and exclusions: on-track $(n=7)$ T2: 1 missing onco-move $(n=8)$

T1 (1 missing for questionnaires, 2 missing physical performance tests) same at T2

UCC $(n=8)$ T1 (1 missing for questionnaires, 2 missing physical performance tests) T2 (none missing for questionnaires, 2 missing for physical performance tests)

Age, mean (SD):

Total $58.2(10.1)$ years

On-track; 57.7 (13.2) years

Onco-move; 60.1 (7.3) years

usual care; 56.7 (10.6)

Gender, n (\%):

Total 14 (61\%) female

On-track; 5 (71\%) female

Onco-move; 3 (38\%) female

Usual care; 6 (75\%) female

Ethnicity: not reported

Comorbidities, n (\%): 15 (65\%) of total participants

SES, $n(\%)$ :

Married $16(70 \%)$ of total

College/university education $11(48 \%)$ of total

Full or part-time work 8 (35\%) of total

Cancer type: colon

Colorectal cancer stage: stage II 3 (13\%); stage III 18 (78\%); stage IV 2 (9\%) - author contacted correspondence received confirmed no stage IV patients were included

Type of treatment:

Larascopic surgery 12 (52\%); control 2 (25\%); on-track 4 (57\%); onco-move 6 (75\%)

Radiotherapy 1 (4\%) control

Chemotherapy 23 (100\%)

Receiving or finished treatment: receiving both interventions started with the first cycle of chemotherapy and continued until 3 weeks after the last cycle

Time beyond treatment: N/A

Time since diagnosis: not reported

\section{Physical activity description}

Onco-move:

Frequency: 5 days a week

Intensity: low-intensity 12-14 on the Borg Scale of Perceived Exertion participants

Time: at least $30 \mathrm{~min}$

Physical activity interventions for disease-related physical and mental health during and following treatment in people with non- 
Waart 2017 (Continued)

Type: not reported

On-track:

Frequency: twice weekly

Intensity: moderate- to high-intensity

Time: six large muscle groups were trained for $20 \mathrm{~min}$ per session in two series of eight repetitions at $80 \%$ of one-repetition maximum, 30 mins of aerobic exercises at an intensity of $50 \%$ to $80 \%$ of the predicted maximal workload of the steep ramp test. Were also encouraged to be physically active 5 days a week for 30 min and to keep an activity diary

Type: resistance and aerobic exercise

Progression: the intensity was further adjusted using the Borg

Length of programme: started with the first cycle of chemotherapy and continued until 3 weeks after the last cycle

Control group: usual care

Setting: onco-move was home based; on-track not specified

Supervised or self-directed: onco-move was unsupervised; on-track was partially supervised

Co-interventions: none

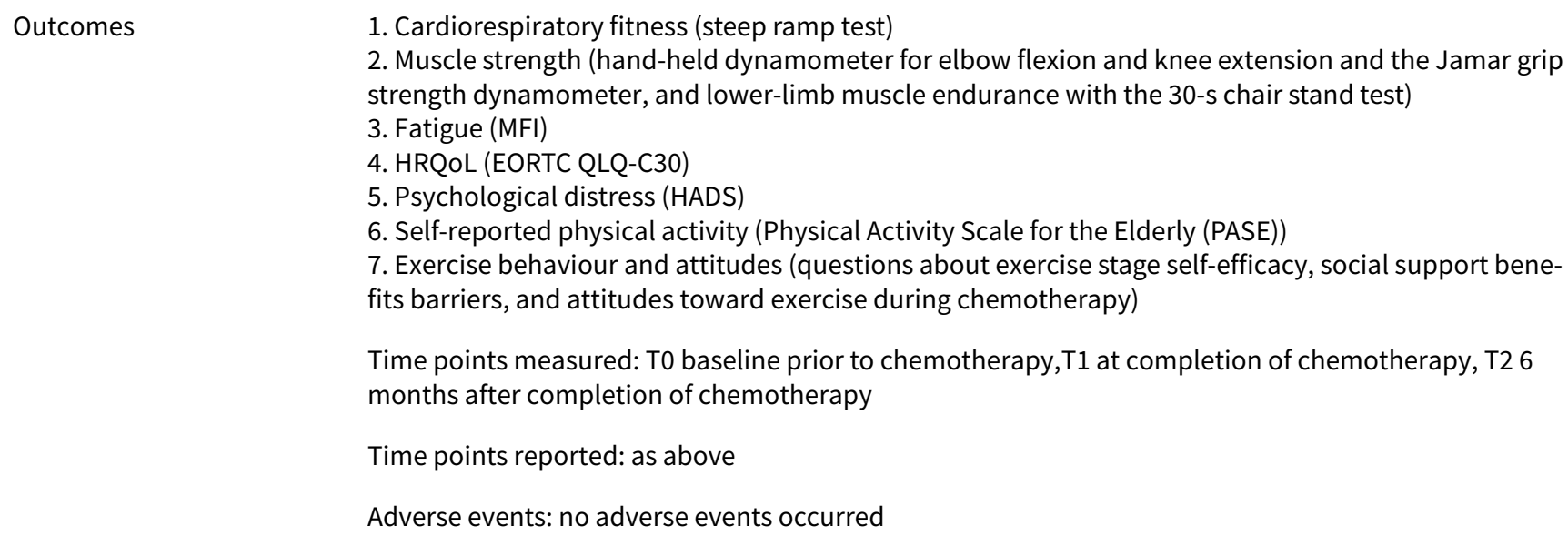
provided by the Dutch Cancer Society and CZ Fund. The interventions were funded by Zilveren Kruis Achmea and The Comprehensive Cancer Centre

\section{Risk of bias}

\begin{tabular}{|c|c|c|}
\hline Bias & Authors' judgement & Support for judgement \\
\hline $\begin{array}{l}\text { Random sequence genera- } \\
\text { tion (selection bias) }\end{array}$ & Low risk & $\begin{array}{l}\text { Randomly assigned to onco-move, on-track, or usual care using the minimisa- } \\
\text { tion method }\end{array}$ \\
\hline $\begin{array}{l}\text { Allocation concealment } \\
\text { (selection bias) }\end{array}$ & Unclear risk & $\begin{array}{l}\text { Whether the treatment assignment was concealed from study personnel and } \\
\text { participants was not described }\end{array}$ \\
\hline $\begin{array}{l}\text { Blinding of participants } \\
\text { and personnel (perfor- } \\
\text { mance bias) } \\
\text { All outcomes }\end{array}$ & High risk & $\begin{array}{l}\text { Not possible to blind participants and personnel due to the nature of the inter- } \\
\text { vention }\end{array}$ \\
\hline $\begin{array}{l}\text { Blinding of outcome as- } \\
\text { sessment (detection bias) } \\
\text { All outcomes }\end{array}$ & Unclear risk & $\begin{array}{l}\text { Insufficient information available to permit a judgement of 'low risk' or 'high } \\
\text { risk' }\end{array}$ \\
\hline
\end{tabular}

Physical activity interventions for disease-related physical and mental health during and following treatment in people with non- 
Waart 2017 (Continued) (attrition bias)

All outcomes

\begin{tabular}{lll}
\hline $\begin{array}{l}\text { Selective reporting (re- } \\
\text { porting bias) }\end{array}$ & High risk & $\begin{array}{l}\text { Study protocol included outcomes (anthropometric measurements, return to } \\
\text { work) data that are not presented in available reports }\end{array}$ \\
\hline Other bias & High risk & Due to baseline imbalances alongside unclear allocation concealment
\end{tabular}

BMI: body mass index; CES-D - Centre for Epidemiological Studies Depression Scale; CRC: colorectal cancer; CTCAE - Common Terminology Criteria for Assessing Adverse Events; ECOG: Eastern Oncology Cooperative Group; EORTC QLQ-C30 - European Organisation for Research and Treatment of Cancer Quality of life; EQ-5D: EuroQol five-dimension scale; FACIT-F - Functional Assessment of Chronic Illness TherapyFatigue; FACT-C - Functional Assessment of Cancer Therapy-Colorectal; FACT-F - Functional Assessment of Cancer Therapy-Fatigue; FACT-G - Functional Assessment of Cancer Therapy-General; HADS - Hospital Anxiety and Depression Scale; ITT: intention-to-treat; MET: metabolic equivalents; MFSI-SF: Multidimensional Fatigue Symptom Inventory- Short Form; PANAS - Positive and Negative Affect Scale; PSQI: Pittsburgh Sleep Quality Index; QoL: quality of life; RCT: randomised controlled trial; SD: standard deviation; SES: socioeconomic status; SF-36 - Medical Outcomes Study Short Form-36 General Health Survey; STAI: State trait anxiety inventory; TOI: trial outcome index; TPAQ - Tartu Physical Activity Questionnaire; UICC: Union for International Cancer Control; UK: United Kingdom; USA: United States of America

\section{Characteristics of excluded studies [ordered by study ID]}

\begin{tabular}{|c|c|}
\hline Study & Reason for exclusion \\
\hline ACTRN12618001855213 & Wrong intervention \\
\hline Adamsen 2009 & Wrong patient population (includes advanced disease and a range of cancer diagnoses) \\
\hline Adlard 2016 & Wrong comparator (moderate- versus high-intensity exercise) \\
\hline Ahn 2013 & Intervention was too short (less than 4 weeks in duration) \\
\hline Allgayer 2004b & Intervention was too short (less than 4 weeks in duration) \\
\hline An 2012 & Intervention was too short (less than 4 weeks in duration) \\
\hline Anderson 2010 & Wrong study design (not a randomised controlled trial) \\
\hline Arving 2013 & Wrong intervention (stress management intervention) for people with a range of cancer diagnoses \\
\hline Backman 2014 & Wrong patient population (includes patients with advanced disease) emailed no reply \\
\hline Basen-Engquist 2015 & Wrong patient population (advanced colorectal cancer) \\
\hline Beck 2015 & Wrong patient population (only one CRC patient included) \\
\hline Beeken 2016 & $\begin{array}{l}\text { Wrong intervention (lifestyle advice on a range of behaviours) for people with a range of cancer di- } \\
\text { agnoses }\end{array}$ \\
\hline Bennett 2007 & Wrong patient population (majority breast cancer patients, other cancer types not specified) \\
\hline Berntsen 2017 & Wrong comparator (moderate- versus high-intensity exercise) \\
\hline Broderick 2014 & Did not analyse CRC patients separately \\
\hline Buffart 2014 & Did not analyse CRC patients separately \\
\hline
\end{tabular}

Physical activity interventions for disease-related physical and mental health during and following treatment in people with non- 


\begin{tabular}{|c|c|}
\hline Study & Reason for exclusion \\
\hline Burnham 2002 & Did not analyse CRC patients separately \\
\hline ChiCTROON15005952 & Wrong study design (not a randomised control trial) \\
\hline Demark-Wahnefried 2012 & Wrong patient population (greater than or equal to 5 years postdiagnosis) \\
\hline DeTroye 2018 & Wrong study design \\
\hline Devin 2016 & Wrong comparator (moderate- versus high-intensity exercise) \\
\hline Dimeo 2004 & Intervention too short (less than 4 weeks in duration) \\
\hline Dodd 2010 & Wrong patient population (only one CRC patient included) \\
\hline DRKS00005793 & wrong comparator (both arms received exercise training) \\
\hline Eakin 2015 & Wrong study design (non-randomised trial) \\
\hline Forbes 2017 & CRC patients not analysed separately - email confirming this \\
\hline Freitag 2018 & Wrong study design \\
\hline Gabrys 2017 & Wrong study design (non-randomised trial) \\
\hline Gillis 2014 & Wrong comparator (prehabilitation versus rehabilitation) \\
\hline Gray 2013 & Intervention was too short (less than 4 weeks in duration) \\
\hline Grimmett 2015 & Wrong study design (feasibility study: non-randomised) \\
\hline Hawkes 2014 & Wrong Intervention (multiple health behaviour change intervention) \\
\hline Hernon 2016 & Wrong patient population (exercise delivered prior to surgery) \\
\hline Hung 2016 & Wrong intervention \\
\hline Ibfelt 2011 & Intervention was too short (6-day retreat) and focused on multiple health behaviours \\
\hline ISRCTN56928944 & Intervention was too short (less than 4 weeks in duration) \\
\hline ISRCTN62859294 & Wrong patient population (exercise intervention delivered prior to surgery) \\
\hline ISRCTN96374224 & Wrong intervention (multiple components: lifestyle and well-being support) \\
\hline Kalter 2015 & CRC not analysed separately \\
\hline Kampshoff 2015 & Did not analyse CRC patients separately \\
\hline Kanera 2017 & Wrong intervention (multiple health behaviour change intervention) \\
\hline laStayo 2011 & Wrong patient population ( $>5$ years postdiagnosis) \\
\hline Lee 2013 & Wrong comparator (both arms received exercise training) \\
\hline Lee $2018 a$ & Dietary co-intervention included \\
\hline
\end{tabular}

Physical activity interventions for disease-related physical and mental health during and following treatment in people with non- 


\begin{tabular}{|c|c|}
\hline Study & Reason for exclusion \\
\hline Ligibel 2012 & $\begin{array}{l}\text { Did not analyse CRC patients separately "the majority of our participants were breast cancer sur- } \\
\text { vivors. We were thus not able to conduct a separate analysis in the colorectal cancer subgroup" }\end{array}$ \\
\hline Lin 2011 & Wrong comparator (both arms received exercise training) \\
\hline Lin 2014 & Wrong study design (non-randomised trial) \\
\hline Lin 2016 & Wrong intervention \\
\hline Loughney 2014 & Wrong patient population (exercise delivered prior to surgery) \\
\hline Lyons 2017 & Wrong patient population (CRC patients not included) \\
\hline Macleod 2018 & Wrong intervention \\
\hline Mayer 2017 & Wrong comparator (control group received a pedometer) \\
\hline McCall 2015 & Wrong comparator (all arms received the yoga intervention) \\
\hline Meyerhardt 2017 & $\begin{array}{l}\text { Outcomes not relevant (email received "Only } 40 \% \text { of participants were colorectal and the end } \\
\text { points were all blood based markers so not sure if this will help your analysis") }\end{array}$ \\
\hline Midtgaard 2011 & Did not analyse CRC patients separately (email confirmation received) \\
\hline Min 2017 & intervention was too short (less than 4 weeks in duration) \\
\hline Morielli 2018 & Wrong intervention \\
\hline NCT00373022 & Wrong comparator (all arms received physical activity) \\
\hline NCT00977613 & Wrong study design (not a randomised controlled trial) \\
\hline NCT00985400 & Wrong patient population (includes stage IV patients) \\
\hline NCT01032590 & Wrong patient population (includes $\mathrm{CRC}>5$ years postdiagnosis) \\
\hline NCT01146769 & Wrong intervention \\
\hline NCT01210313 & Wrong study design (observational) \\
\hline NCT01325909 & Wrong study design (non-randomised trial) \\
\hline NCT01453452 & Wrong study design (non-randomised trial) \\
\hline NCT01991847 & Wrong study design (non-randomised trial) \\
\hline NCT02052050 & Wrong study design (non-randomised trial) \\
\hline NCT02056691 & Wrong study design (non-randomised trial) \\
\hline NCT02264496 & Wrong patient population (exercise delivered prior to surgery) \\
\hline NCT02442583 & Wrong study design (non-randomised trial) \\
\hline NCT02512263 & Study was not carried out \\
\hline
\end{tabular}

Physical activity interventions for disease-related physical and mental health during and following treatment in people with non- 


\begin{tabular}{|c|c|}
\hline Study & Reason for exclusion \\
\hline NCT02522520 & Wrong comparator (both arms received a pedometer intervention) \\
\hline NCT02586701 & Wrong comparator (both arms received exercise training) \\
\hline NCT02647398 & Wrong comparator (both arms received exercise) \\
\hline NCT02677389 & Wrong comparator (both arms received physical activity) \\
\hline NCT02837159 & Study has been suspended due to financing issues \\
\hline NCT02889276 & Wrong comparator (both arms received physical activity) \\
\hline NCT03036436 & Wrong study design (non-randomised trial) \\
\hline NCT03082495 & Wrong patient population (exercise during neoadjuvant treatment) \\
\hline NCT03120104 & Wrong comparator \\
\hline NCT03232814 & Wrong study design (non-randomised trial) \\
\hline NCT03630354 & Wrong comparator \\
\hline NTR6383 & Wrong intervention \\
\hline Oh 2008 & $\begin{array}{l}\text { Wrong intervention and wrong patient population (mixed cancer diagnoses - three CRC patients in- } \\
\text { cluded, all in the control arm) }\end{array}$ \\
\hline Onerup 2017 & Wrong patient population (exercise intervention delivered pre- and postsurgery) \\
\hline Phipps 2018 & Wrong study design \\
\hline Piringer 2013 & Wrong study design (non-randomised trial) \\
\hline Piringer 2018 & Wrong study design \\
\hline Ratjen 2018 & Wrong intervention \\
\hline Ray 2018 & Wrong study design \\
\hline Sandler 2017 & Wrong intervention (intervention group received CBT along with exercise) mixed cancer diagnoses \\
\hline Sellar 2014 & Wrong study design (non-randomised trial) \\
\hline Sohl 2016 & Intervention was too short (less than 4 weeks in duration) \\
\hline Sprod 2015 & Wrong patient population (less than $3 \%$ of patients had gastrointestinal cancers) \\
\hline Ungar 2016 & Wrong comparator \\
\hline van Rooijen 2018 & Wrong study design \\
\hline Wang 2012 & Intervention was too short (less than 4 weeks in duration) \\
\hline West 2015 & Wrong patient population (exercise intervention delivered prior to surgery) \\
\hline
\end{tabular}




\begin{tabular}{ll}
\hline Study & Reason for exclusion \\
\hline Zopf 2018 & Wrong study design \\
\hline
\end{tabular}

Characteristics of studies awaiting classification [ordered by study ID]

\section{De Backer 2007}

\begin{tabular}{ll}
\hline Methods & RCT \\
\hline Participants & $\begin{array}{l}57 \text { patients (age } 24 \text { to } 73 \text { years) who had received chemotherapy for lymphomas, breast, gynaeco- } \\
\text { logic, testicular, or colorectal cancer (8 CRC participants) }\end{array}$
\end{tabular}

Interventions Intervention details: 18-week high-intensity strength training programme in cancer survivors Comparator details: standard care

Outcomes
2. Cardiopulmonary function $\left(\mathrm{VO}_{2}\right.$ max)
3. Maximal short exercise capacity (MSEC)
4. Body composition
5. Health-related quality of life (HRQOL)

Notes Emailed to check whether CRC patients analysed separately

Golsteijn 2017

\begin{tabular}{ll}
\hline Methods & RCT \\
\hline Participants & 510 prostate and colorectal cancer patients (during treatment or up to 1 year post-treatment) \\
\hline Interventions & $\begin{array}{l}\text { Intervention details: onco-active (computer-tailored physical activity intervention participants re- } \\
\text { ceived tailored advice at } 3 \text { time points, a pedometer and access to interactive content on the web- } \\
\text { site) }\end{array}$ \\
Comparator: usual care waiting list control
\end{tabular}

\section{Outcomes}

1. Physical activity behaviour (self-report Short Questionnaire to Assess Health Enhancing Physical Activity (SQUASH) and accelerometer data) intention be physically active,

\section{HRQOL (EORTC QLQ-C30) \\ 3. Fatigue (Checklist Individual Strength)}

Notes Emailed: data may be shared

Ho 2013

\begin{tabular}{ll}
\hline Methods & RCT \\
\hline Participants & $\begin{array}{l}222 \text { colorectal cancer patients who completed curative treatment without evidence of recurrence } \\
\text { will be recruited into the study }\end{array}$ \\
\hline
\end{tabular}

Physical activity interventions for disease-related physical and mental health during and following treatment in people with non- 
Ho 2013 (Continued)

Interventions intervention:

Arm 1: dietary intervention ( $<5$ servings of red/processed meat weekly, 2 servings of refined grains daily)

Arm 2: physical activity intervention (general health target - 30 minutes moderate to vigorous physical activity, 5 days per week (i.e. 10 MET-hours/week); cancer outcome target - 60 minutes of moderate to vigorous physical activity 5 days per week (i.e. 18-20 MET-hours/week))

Arm 3: dietary and physical activity intervention (meeting both physical activity and dietary targets)

comparator: usual care

1. Whether physical activity and dietary targets are met
2. Magnitude and mechanism of behavioural change
3. The degree and determinants of compliance
4. The additional health benefits and side effects of the intervention

Notes

Estimated study completion date: June 2017 emailed for information, no reply. Trial registration number: NCT01708824

Houborg 2006

\begin{tabular}{ll}
\hline Methods & RCT \\
\hline Participants & 119 patients with colorectal cancer (96), diverticulitis (7) and non-malignant disease (16) \\
\hline Interventions & $\begin{array}{l}\text { Intervention details: demanding exercises covering mobilisation, strength training of upper and } \\
\text { lower extremities, and aerobic training } \\
\text { comparator: performed relaxation exercises and received hot wrappings and massage }\end{array}$ \\
\hline Outcomes & $\begin{array}{l}\text { 1. Fatigue (visual analogue scale) } \\
\text { 2. Muscular strength } \\
\text { 3. Walking speed } \\
\text { 4. Physical performance test } \\
\text { 5. Physical function questions (SF-36) }\end{array}$ \\
\hline
\end{tabular}

\section{ISRCTN07465566}

\begin{tabular}{ll}
\hline Methods & RCT \\
\hline Participants & Adults with CRC, Duke stage A-C2, completed surgery in the past 12 months \\
\hline Interventions & $\begin{array}{l}\text { Intervention details: physical activity consultation after baseline (lasting 1-2 hours) and one at } 3 \\
\text { months }\end{array}$
\end{tabular}

Physical activity interventions for disease-related physical and mental health during and following treatment in people with non- 
ISRCTN07465566 (Continued)

Comparator: usual care

1. Feasibility (measured by rates of recruitment, completion, adherence),
2. Change in physical activity (Actigraph)
3. Mental well-being (HADS)
4. QoL (FACT-G and FACT-C)
5. Body composition
6. Predictors of change in physical activity
7. Perceptions of cancer risk in partners
8. Fear of cancer recurrence
$\begin{aligned} & \text { 9. Key components of the revised theory of planner behaviour will be measured in individuals living } \\ & \text { with colorectal cancer and their partner. }\end{aligned}$
$\begin{aligned} & \text { 10. The quality of the relationship between the individual living with cancer and their partner will } \\ & \text { be measured to test if it affects adherence to the physical activity intervention or moderates any of } \\ & \text { the intervention effects }\end{aligned}$
Emailed - no reply received

\section{Jacobsen 2013}

\begin{tabular}{ll}
\hline Methods & RCT \\
\hline Participants & $\begin{array}{l}460 \text { cancer patients receiving chemotherapy representing a range of cancer diagnoses (15 colon } \\
\text { cancer, } 6 \text { rectal/anal cancer) }\end{array}$ \\
\hline
\end{tabular}

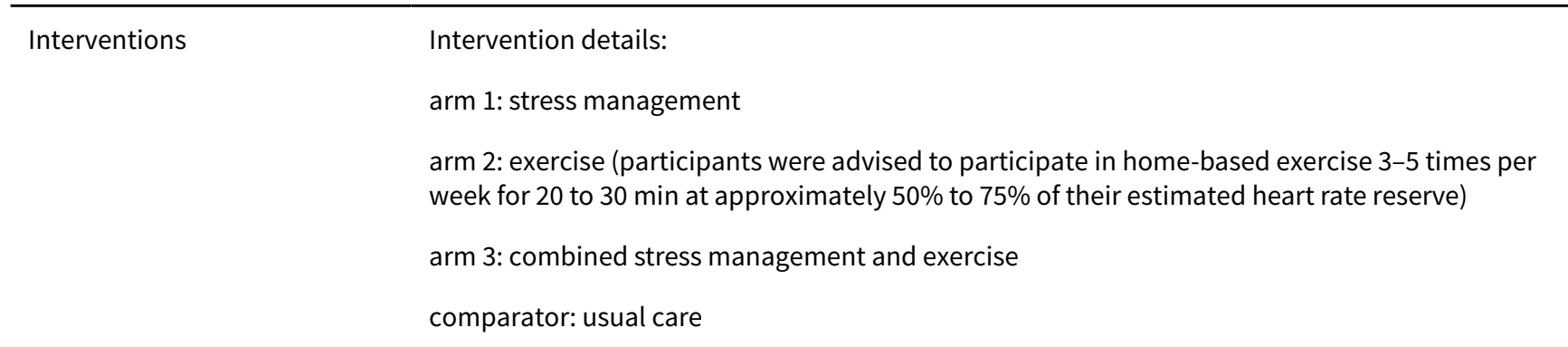

\begin{tabular}{ll}
\hline Outcomes & 1. Mental and physical well-being \\
2. Depression and anxiety \\
3. Exercise \\
4. Stress reduction activity \\
\hline Notes & Unable to contact via email \\
\hline
\end{tabular}


Lee 2018 (Continued)

Participants $\quad 72$ colon and rectal cancer patients (38 intervention; 34 usual care)

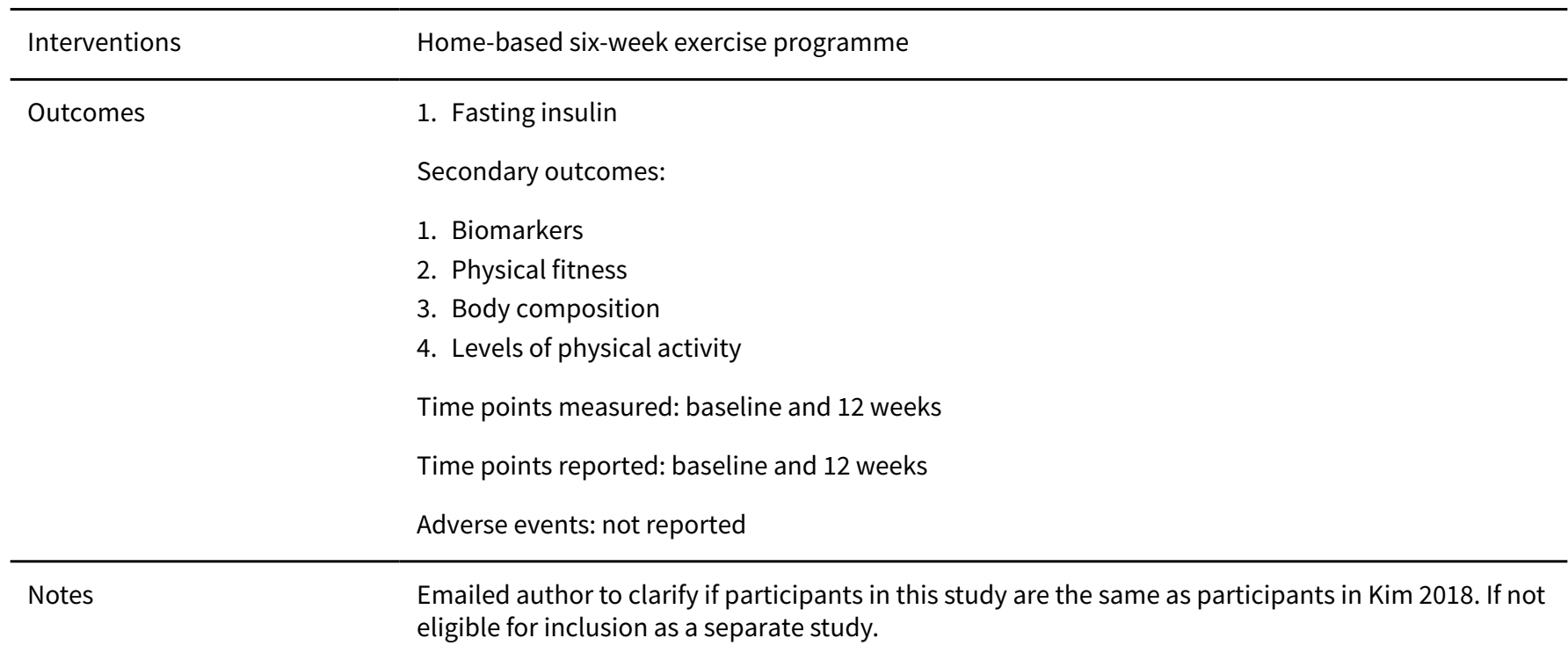

Maxwell-Smith 2019

\begin{tabular}{|c|c|}
\hline Methods & $\mathrm{RCT}$ \\
\hline Participants & Colorectal or endometrial cancer patients at cardiovascular risk \\
\hline \multirow[t]{2}{*}{ Interventions } & Intervention details: a wearable tracker, two group sessions and a support phone call \\
\hline & Comparator: received printed materials describing physical activity guidelines \\
\hline \multirow[t]{4}{*}{ Outcomes } & 1. Moderate to vigorous physical activity \\
\hline & 2. Blood pressure \\
\hline & 3. BMI \\
\hline & 4. Sedentary behaviour \\
\hline Notes & Emailed requesting colorectal cancer patient data separately \\
\hline
\end{tabular}

Moller 2015

\begin{tabular}{ll}
\hline Methods & RCT \\
\hline Participants & breast or colon (33\%) cancer patients \\
\hline Interventions & Intervention details \\
& $\begin{array}{l}\text { arm 1: } 12 \text {-week hospital-based, supervised group exercise intervention of moderate- to high-inten- } \\
\text { sity three times weekly, health promotion counselling and symptom management coaching or } \\
\text { arm 2: } 12 \text { week home-based individual pedometer programme, health promotion counselling and } \\
\text { symptom management }\end{array}$
\end{tabular}


Moller 2015 (Continued)

comparator: a control group

\begin{tabular}{|c|c|}
\hline \multirow[t]{8}{*}{ Outcomes } & 1. Maximal oxygen uptake $\left(\mathrm{VO}_{2}\right.$ peak) \\
\hline & 2. Psychological well-being (HADS) \\
\hline & 3. QOL (EORTC QLQ-C30) \\
\hline & 4. General well-being (SF-36) \\
\hline & 5. Perception of pain (Brief Pain Inventory) \\
\hline & 6. Leisure time physical activity level (self-reported) \\
\hline & $\begin{array}{l}\text { 7. Motivational readiness and ability and barriers to engage in exercise activities 'stages of motiva- } \\
\text { tional readiness' 'decisional balance' and 'exercise self-efficacy' (using validated questionnaires), }\end{array}$ \\
\hline & 8. Social support and network (Multidimensional Scale of Perceived Social Support) \\
\hline Notes & Trial registration number: ISRCTN24901641 Emailed \\
\hline
\end{tabular}

\section{NCT02403024}

\begin{tabular}{|c|c|}
\hline Methods & $\mathrm{RCT}$ \\
\hline Participants & $\begin{array}{l}42 \text { CRC patients who had completed surgery for local stage disease and patients who had complet- } \\
\text { ed surgery and any adjuvant chemotherapy for locally advanced stage disease }\end{array}$ \\
\hline Interventions & $\begin{array}{l}\text { Intervention details: home-based walking intervention (150 minutes per week) } \\
\text { comparator: usual care (waiting list control) }\end{array}$ \\
\hline Outcomes & $\begin{array}{l}\text { 1. Cardiopulmonary fitness }\left(\mathrm{VO}_{2} \text { peak during CPET test) }\right. \\
\text { 2. Feasibility (inclusion rate, attrition rate, adherence) } \\
\text { 3. Body composition (DXA scan) } \\
\text { 4. Blood biochemistry (fasting blood samples) } \\
\text { 5. Glycaemic control (oral glucose tolerance test) } \\
\text { 6. Patient reported outcome measures (health-related quality of life by FACT-C, sleep quality by } \\
\text { Pittsburgh Sleep Quality Index and exercise motivation by Behavioral Exercise Regulations Ques- } \\
\text { tionnaire) }\end{array}$ \\
\hline Notes & $\begin{array}{l}\text { The "Interval Walking in Colorectal Cancer" (I-WALK-CRC) study: design, methods and recruitment } \\
\text { results of a randomised controlled feasibility trial- Publication } 2018\end{array}$ \\
\hline
\end{tabular}

\section{NCT02564835}

\begin{tabular}{ll}
\hline Methods & RCT \\
\hline Participants & 27 CRC survivors (any stage) \\
\hline Interventions & Intervention details:
\end{tabular}


arm 2: physical activity - two 90-minute classes every week for a total of 12 weeks, nine resistance exercises (e.g. squats) and 8 flexibility exercises (e.g. shoulder stretch) targeted for the whole body as well as a brief warm up and cool down (walking)

comparator: usual care

1. Program evaluation (participant experience and satisfaction with
2. Attention Network Test, attention and cognitive control (Digit Spares
Test, Trail Making Test, Attentional Function Index),
3. Functional Assessment of Cancer Therapy - Cognitive Function
4. Pro-inflammatory Markers
5. attendance, Adherence and Attrition Log
6. Safety Log
7. Demographic and Medical Information Questionnaires
8. Profile of Mood States-Brief Form
9. Mindful Attention Awareness Scale, Beliefs about Treatment

Notes NCT02564835

\section{Park 2015}

\begin{tabular}{ll}
\hline Methods & RCT \\
\hline Participants & $\begin{array}{l}162 \text { early stage breast }(n=122,75.3 \%) \text { and colorectal cancer survivors }(n=40,24.7 \%) \text { who com- } \\
\text { pleted primary and adjuvant treatments }\end{array}$ \\
\hline
\end{tabular}

Interventions Intervention details:

arm 1: oncologists' exercise recommendation

arm 2: oncologists' exercise recommendation with exercise motivation package (pedometer, exercise education and exercise diary)

comparator: control
Outcomes
1. Amount of exercise participation (Godin LeisureTime Exercise Questionnaire)
2. Quality of life
3. Self-reported physical functioning (EORTC QLQ-C30)

Notes

Methods RCT? There is a control group and exercise group, report does not specify whether participants are
randomly allocated 
Schulz 2002 (Continued)

Participants

Interventions

49 colon cancer patients; 24 control, 25 intervention

Intervention details: ergometer exercise training performed once a day for approximately $40 \mathrm{~min}$ utes, 5 days per week for 5 weeks at a moderate training level

Comparator: control group

Outcomes 1. Psychological parameters (EORTC QLQ-C30, FPI-R)

2. Immunological parameters

Notes UTC via email

Schwartz 2009

\begin{tabular}{|c|c|}
\hline Methods & $\mathrm{RCT}$ \\
\hline Participants & 112 females with breast, lymphoma and colon cancer \\
\hline Interventions & $\begin{array}{l}\text { Intervention details: } \\
\text { arm 1: } 12 \text { months, aerobic exercise, moderate-intensity home-based exercise } 4 \text { days per week. The } \\
\text { duration of each specific exercise programme ranged from } 20 \text { to } 30 \text { minutes } \\
\text { arm 2: } 12 \text { months, resistance exercise home-based exercise } 4 \text { days per week. The duration of each } \\
\text { specific exercise programme ranged from } 20 \text { to } 30 \text { minutes. } \\
\text { comparator: usual care control }\end{array}$ \\
\hline Outcomes & $\begin{array}{l}\text { 1. Body weight } \\
\text { 2. Aerobic capacity (12-minute walk) } \\
\text { 3. Muscle strength (1-repetition maximum tests) } \\
\text { 4. Body fat (dual energy x-ray absorptiometry scans) }\end{array}$ \\
\hline Notes & UTC via email \\
\hline
\end{tabular}

Shun 2016

\begin{tabular}{ll}
\hline Methods & RCT \\
\hline Participants & 128 postsurgical colorectal cancer patients; 65 control, 63 intervention \\
\hline Interventions & $\begin{array}{l}\text { Intervention details: } 12-\text { week home-based moderate-intensity exercise programme } \\
\text { Comparator: usual care, maintained their normal daily activity }\end{array}$ \\
\hline Outcomes & 1. Fatigue \\
\hline Notes & 2. Psychological distress (depression) \\
\hline
\end{tabular}

Physical activity interventions for disease-related physical and mental health during and following treatment in people with non- 
BMI: body mass index; CRC: colorectal cancer; EORTC QLQ-C30 - European Organisation for Research and Treatment of Cancer Quality of life; FACT-C - Functional Assessment of Cancer Therapy-Colorectal; FACT-G - Functional Assessment of Cancer Therapy-General; FPIR- Freiburg Personality Inentory; HADS - Hospital Anxiety and Depression Scale; HRQoL- health related quality of life; MET: metabolic equivalents; QoL: quality of life; RCT: randomised controlled trial; SF-36 - Medical Outcomes Study Short Form-36 General Health Survey; UTC- unable to contact

Characteristics of ongoing studies [ordered by study ID]

\section{ChiCTRIOR17012037}

Study name The impact of resistance training combined with aerobic exercises on the functional recovery and
quality of life of colorectal cancer survivors postsurgeries

$\begin{array}{ll}\text { Methods } & \text { Study design: Randomised parallel controlled trial } \\ \text { Country where study is being conducted: China } & \text { Inclusion criteria: } \\ \text { Participants } & \begin{array}{l}\text { 1. clinically diagnosed as colorectal cancer in stage I to III by the TNM staging system } \\ \text { 2. waiting for surgical treatment } \\ \text { 3. willing to participate in the study }\end{array}\end{array}$

\section{Exclusion criteria:}

1. secondary or recurrent cancer

2. serious neurological or cardiovascular conditions (e.g. recent stroke)

3. severe mental or cognitive disorders

4. other illnesses and contraindications to exercise

Interventions

Intervention details: postoperative cancer survivors in the experimental group would participate in low-intensity resistance and aerobic exercise for 7 days, supervised by an experienced physiotherapist. And then the patients would follow a 12-week moderate- to high-intensity home exercise programme

Comparator details: will only receive conventional treatment

\begin{tabular}{ll}
\hline Outcomes & 1. The FACIT (Functional Assessment of Chronic Illness Therapy) measurement system \\
2. Six-minute walk test & 3. muscle strength of major muscles in upper and lower limbs \\
& Time points measured: day 1 preoperation, day 7 postoperation, month 3 postoperation \\
\hline Starting date & 18 May 2016 \\
\hline Contact information & Yuling Wang \\
& Tel: +8613054445587 \\
& Email: wangyul@mail.sysu.edu.cn \\
\hline Notes & Trial registration identifier: ChiCTR-IOR-17012037 \\
Trial registration link: http://www.chictr.org.cn/showprojen.aspx?proj=20476 \\
Sponsor: Department of Rehabilitation Medicine, The Sixth Affiliated Hospital of Sun Yat-sen Uni- \\
versity
\end{tabular}


Methods

Study design: RCT

Country where study is being conducted: USA

Participants

\section{Inclusion criteria:}

1. $\geq$ Age 60 years (no upper age limit)

2. Diagnosis of stage II-III colon or rectal cancer planned for treatment with adjuvant chemotherapy scheduled as part of standard treatment

3. Able to read English

4. Approval from their treating physician to engage in moderate-intensity physical activity

5. Patient-assessed ability to walk and engage in moderate physical activity

6. Signed, IRB-approved written informed consent

\section{Exclusion criteria:}

1. Other active, invasive malignancy requiring ongoing therapy or expected to require systemic therapy

2. Already walking or engaging in other physical activity $>120$ minutes per week as documented via subject self-report

3. Unable to walk or engage in moderate-intensity physical activity

4. One or more significant medical conditions that in the physician's judgment preclude participation in the walking intervention

Intervention details: participants will take part in the Walk With Ease (WWE) programme during the course of their chemotherapy treatment. They will be requested to initiate the WWE starting on Day 1 of adjuvant chemotherapy. Participants are asked to walk at a safe and comfortable pace, increasing their minutes per day at a rate they can sustain, with the ultimate goal of 30 minutes/day for at least 5 days/week. They are asked to maintain a daily walking log that is provided to them, entering total minutes per day.

Participants will be asked to do the walking programme independently (self-directed, not in a formal group with an instructor) throughout chemotherapy.

Comparator details: standard care

Outcomes

Primary outcome measures:

1. change in fatigue (measured via PROMIS-Fatigue)

2. changes in physical function (measured via PROMIS-PF and SPPB)

3. adherence to the physical activity intervention (measured through self-reported minutes of walking per days and walking minutes per week)

4. difference in p16INK4a levels (change in p16INK4a and muscle mass measurements pre-/postadjuvant chemotherapy)

5. change in muscle mass measurements (change in p16INK4a and muscle mass measurements)

6. association of p16INK4a and muscle mass with any differences in fatigue, physical function or QoL during chemotherapy

7. quality of life (FACT-FCSI and PROMIS-PI)

8. changes in activities of daily living

9. changes in instrumental activities of daily living

10.changes in self-efficacy (measured by PSEFSM and OEE)

Time points measured: baseline, 3 months, and after completion of chemotherapy (24 weeks), some outcomes measured at 1 year 
NCT02191969 (Continued)

Starting date June 2014
Kirsten A Nyrop knyrop@med.unc.edu

Amy L Garrrett amy_garrett@med.unc.edu

Trial registration identifier:NCT02191969

Trial registration link: https://clinicaltrials.gov/ct2/show/NCT02191969

Sponsor: UNC Lineberger Comprehensive Cancer Center

\section{NCT02538913}

\begin{tabular}{ll}
\hline Study name & Exercise training for rectal cancer patients. A randomized \\
\hline Methods & Study design: RCT \\
Country where study is being conducted: Norway & 150 people with rectal cancer \\
Inclusion criteria: \\
1. Rectal cancer \\
2. Planned curative LAR with preoperative radiotherapy \\
3. Cancer stadium I-III \\
4. Able to speak and understand Norwegian
\end{tabular}

\section{Exclusion criteria:}

1. Previous radiotherapy

2. Previous pelvic surgery

3. Diseases affecting the anal sphincter

Interventions

Intervention details: daily pelvic floor muscle training and individualised regular exercise training (aerobic and strength exercise) three days per week.

Comparator details: standard care

\section{Outcomes}

Primary outcome:

1. Anal incontinence ( 3 and 12 months postsurgery)

Secondary outcomes:

1. Urinary incontinence ( 3 and 12 months postsurgery)

2. Bowel and sexual dysfunction ( 3 and 12 months postsurgery)

3. Physiology of the anal sphincter ( 3 and 12 months postsurgery)

4. Quality of life (3 and 12 months postsurgery)

5. Maximal oxygen uptake $\left(\mathrm{VO}_{2} \max \right)$ (1 week pre-surgery)

6. Postoperative complications (up to 5 years postsurgery)

7. Physical activity level ( 1 week pre-surgery and 3 months postsurgery)

8. In-hospital time (up to 12 months postsurgery)

Time points measured: multiple time points measured see above 
NCT02538913 (Continued)

Starting date September 2015

\section{Contact information}

Notes
Signe N Stafne, PhD, +47 48071 766, signe.n.stafne@ntnu.no

Trial registration identifier: NCT02538913

Trial registration link: https://clinicaltrials.gov/ct2/show/NCT02538913

Sponsor: Norwegian University of Science and Technology

\section{NCT03064308}

Study name

Study design: RCT

Country where study is being conducted: UK

Participants

30 participants undergoing major body surgery including those with colorectal carcinoma

\section{Inclusion criteria:}

1. Male and Female patients

2. Age greater than 70 years

3. Patients undergoing neoadjuvant chemotherapy

\section{Exclusion criteria:}

1. Participation in a formal exercise regime

2. $A B M I>35 \mathrm{~kg} / \mathrm{m} 2$

3. Active cardiovascular disease: uncontrolled hypertension (BP $>180 / 100)$, angina, heart failure (class III/IV), arrhythmia, right to left cardiac shunt, recent cardiac event

4. Taking beta-adrenergic blocking agents

5. Cerebrovascular disease: untreated aneurysm (large vessel or intracranial)

6. Respiratory disease including: pulmonary hypertension

7. Metabolic disease: hyper and hypoparathyroidism, untreated hyper and hypothyroidism, Cushing's disease

8. Musculoskeletal or neurological disorders

9. Family history of early ( $<55$ years) death from cardiovascular disease

10.Severe rheumatoid arthritis limiting ability to perform any part of the assessment process

11. Unable to complete the consent process

Interventions Intervention details: participants will complete a High-Intensity Functional Exercise (HIFE) programme.

Comparator details: standard NHS care

Outcomes

Primary:

1. Feasibility of a home-based exercise programme following surgery in the older patient

Secondary:

1. Maximal oxygen uptake $\left(\mathrm{VO}_{2}\right.$ max $)$

2. Muscle architecture ultrasound

Physical activity interventions for disease-related physical and mental health during and following treatment in people with non- 
NCT03064308 (Continued)
3. Cognitive testing
4. Frailty
5. Physical activity
6. Quality of life
7. Hand strength test
8. Intervention compliance questionnaire

Time points measured: baseline, 3 and 6 months

\begin{tabular}{ll}
\hline Starting date & 26 June 2017 \\
\hline Contact information & Laura Carrick, BSc MBChB 07429377430 msxlc2@nottingham.ac.uk \\
& Bethan Phillips, BSc PhD 01332724731 \\
\hline Notes & Trial registration number: NCT03064308 \\
& Trial registration link: https://clinicaltrials.gov/ct2/show/NCT03064308 \\
& Sponsor: University of Nottingham \\
\hline
\end{tabular}

NCT03210129

\begin{tabular}{ll}
\hline Study name & Motivational Interviewing to Increase Physical Activity behaviour in Cancer patients (MIPAClux) \\
\hline Methods & Study design: RCT \\
& Country where study is being conducted: Luxembourg \\
\hline
\end{tabular}

Participants 70 breast, endometrial or colorectal cancer participants

\section{Inclusion:}

1. cancer stage $\leq$ stage III

2. $>3$ months after primary treatment

3. $<24$ months after primary treatment

4. ECOG performance score $<2$

5. signed Informed consent

\section{Exclusion:}

1. recurrent cancer

2. history of other types of cancer

3. second primary tumour

4. planned surgery within the duration of the study

5. known or obvious cognitive or psychiatric impairments

6. positive pregnancy test

Interventions

Intervention details: 12 motivational interviewing sessions exploring self-assessed confidence, ambivalence, and personal values concerning changes in active lifestyle

Comparator details: standard care

Outcomes

Primary outcome:

1. Change in physical activity behaviour 
NCT03210129 (Continued)

Secondary outcomes:

1. Body mass index

2. Physical fitness

3. Cost-effectiveness

Time points measured: weeks 1,14 and 26

\begin{tabular}{ll}
\hline Starting date & 6 July 2017 \\
\hline Contact information & Alexis Lion, PhD, +35226970849 alexis.lion@lih.lu \\
& Eric Besenius, M.Sc., +35226970917 \\
\hline Notes & Trial registration number: \\
& Trial registration link: https://clinicaltrials.gov/ct2/show/NCT03210129 \\
& Sponsor: Luxembourg Institute of Health \\
\hline
\end{tabular}

NCT03212079

Study name Novel individualized intervention for behavioral change among high-risk group cancer survivors: Physical Activities by Technology Help (PATH)

\begin{tabular}{ll}
\hline Methods & Study design: RCT \\
Country where study is being conducted: USA
\end{tabular}

\section{Participants}

42 cancer survivors of breast, prostate, lung, colorectal, cervical or oral cancer survivor

\section{Inclusion:}

1. Finished active cancer treatment at least three months ago

2. Overweight or obese and do not exercise daily

3. Do not have any physical limitation to do mild to moderate physical activities

4. Have a smart phone and actively use an email account

5. Willing to sign the consent form

\section{Exclusion:}

1. Already doing moderate to high physical activities in their daily life (rapid screener)

2. Planning to relocate within the next 4-5 weeks

3. Stage 4 cancer

4. Already using physical activity tracker or part of a physical activity programme

5. Part of another study that may interfere with our outcome of interest, unstable mental condition

6. Mental condition that prevents patient from performing the study activities and requirements

7. Pregnancy

Interventions Intervention details:

Arm 1: Mycoach Smart Text - personalised text messages to cell phone to help participants to increase activity

Arm 2: MyCoach via Amazon Alexa- will interact with intelligent coach to increase activity

Comparator details: control participants will self-motivate to increase physical activities

Physical activity interventions for disease-related physical and mental health during and following treatment in people with non- 
NCT03212079 (Continued)

Primary outcome:
1. Average number of steps
Secondary outcomes:
1. Total number and duration of activity bouts
2. Transitions between active/inactive periods
3. Daily patterns of activity
Time points measured: 5 weeks

\begin{tabular}{ll}
\hline Starting date & 3 April 2017 \\
\hline Contact information & Ahmed Hassoon, MD, MPH, PMP 4432872775 ahassoo1@jhu.edu \\
\hline Notes & Trial registration number: NCT03212079 \\
& Trial registration link: https://clinicaltrials.gov/ct2/show/NCT03212079 \\
& Sponsor: John Hopins University \\
\hline
\end{tabular}

$\begin{array}{ll}\text { Methods } & \text { Study design: RCT } \\ \text { Country where trial is being conducted: Belgium }\end{array}$

\section{Participants}

\section{Inclusion criteria:}

1. primary diagnosis of prostate cancer, head and neck cancer (oral cavity, pharynx, larynx, nasal cavity/sinuses, or salivary gland) or rectum cancer

2. age over 18 years

3. no distant metastases and/or disease progression

4. at least 25 scheduled radiation treatments ( 5 weeks)

5. ability to read, write, and speak French

\section{Exclusion criteria:}

1. uncontrolled cardiac, hypertensive or pulmonary diseases

2. uncontrolled insulin-dependent diabetes mellitus

3. neuropsychiatric disorders or orthopaedic conditions that would impair exercise participation

4. abnormal electrocardiogram

\section{Intervention details}

Arm 1: high-intensity aerobic training group will be conducted on cycle ergometers or treadmill with heart rate measured throughout each session

Arm 2: resistance training group muscle strengthening will incorporate eight exercises targeting major muscle groups at $60 \%$ to $85 \%$ of their estimated 1-repetition maximum (1RM)

Comparator details: no intervention, usual care

Outcomes Primary outcome measures:

Physical activity interventions for disease-related physical and mental health during and following treatment in people with non- 
NCT03252821 (Continued)

1. Change in fatigue (FACIT-fatigue questionnaire)

Secondary outcome measures

1. Change in functional capacity (6-minute walk test)

2. Change in quality of life (Functional Assessment of Cancer Therapy-General)

3. Change in sleep disturbances (Pittsburgh Sleep Quality Index)

4. Change in somnolence syndrome (Epworth Sleepiness Scale)

5. Change in insomnia (Insomnia Severity Index)

6. Change in depression symptoms (Center for Epidemiologic Studies Depression Scale)

7. Change in executive functions (Trail Making Test)

8. Change in dyspnoea (Multidimensional Dyspnea Profile)

9. Adherence (Percentage of completed sessions)

Time points measured: baseline, 5 weeks for rectum cancer

\begin{tabular}{ll}
\hline Starting date & 15 August 2017 \\
\hline Contact information & Gilles Caty gilles.caty@uclouvain.be \\
& Elise Piraux elise.piraux@uclouvain.be \\
\hline Notes & Trial registration identifier: NCT03252821 \\
& Trial registration link: https://clinicaltrials.gov/ct2/show/NCT03252821 \\
& Sponsor: Cliniques universities Saint-Luc- Université Catholique de Louvain \\
\hline
\end{tabular}

\section{NCT03885817}

\begin{tabular}{ll}
\hline Study name & Physically active during cancer treatment (FAKT) \\
\hline Methods & Study design: RCT \\
& Country where study is being conducted: Norway
\end{tabular}

\begin{tabular}{ll}
\hline Participants & 64 participants with CRC radical resection within past 3 months \\
\hline Interventions & Intervention details: exercise training and nutritional guide \\
& Comparator details: usual care \\
\hline
\end{tabular}

Outcomes Primary:

1. Patient-reported peripheral sensory neuropathy

Secondary:

1. Fatigue

2. Change in patient reported peripheral sensory, autonomic and motor neuropathy

3. Change in BMI and body composition

4. Change in blood pressure and Tx of hypertension, cholesterol, glucose

5. Change in nutritional status

6. Change in QOL

7. Physical capacity (balance, muscle strength aerobic capacity)

8. Levels of physical activity

9. Overall- and disease-free survival

Physical activity interventions for disease-related physical and mental health during and following treatment in people with nonadvanced colorectal cancer (Review)

Copyright $\odot 2020$ The Cochrane Collaboration. Published by John Wiley \& Sons, Ltd. 
NCT03885817 (Continued)

10. Time before return to work

11. Haematological toxicity

12.Adverse events

13. Patient experience

14.Diagnosed with type 2 diabetes

\begin{tabular}{ll}
\hline Starting date & Not described - not yet recruiting \\
\hline Contact information & $\begin{array}{l}\text { Contact: Ingunn Hatlevoll, }+4790866361 \\
\text { ingunn.hatlevoll@stolav.no } \\
\text { Contact: Eva Hofsli, MD PhD } \\
\text { eva.hofsli@ntnu.no }\end{array}$ \\
\hline Notes & NCT03885817 \\
\hline
\end{tabular}

\section{Piringer 2017}

Study name

Phase III randomised trial of endurance exercise following adjuvant chemotherapy for colorectal cancer "aBCSG C08-II trial"

\begin{tabular}{ll}
\hline Methods & Study design: RCT \\
& Country where study is being conducted: Austria \\
\hline Participants & $\begin{array}{l}788 \text { patients with newly diagnosed, locally advanced colorectal cancer after adjuvant chemothera- } \\
\text { py }\end{array}$
\end{tabular}

Interventions

Intervention details: one year endurance exercise intervention

Comparator details: usual care while maintaining habitual physical activity levels

\begin{tabular}{|c|c|}
\hline Outcomes & $\begin{array}{l}\text { Primary: } \\
\text { 1. Disease-free survival } \\
\text { Secondary: } \\
\text { 1. Recurrence-free survival } \\
\text { 2. Overall survival } \\
\text { 3. Physical activity end points } \\
\text { 4. PROMS } \\
\text { 5. Cost-effectiveness } \\
\text { 6. Utility and safety } \\
\text { Time points measured: not reported }\end{array}$ \\
\hline Starting date & Not known \\
\hline Contact information & Not known \\
\hline
\end{tabular}

BMI: body mass index; ECOG: Eastern Oncology Cooperative Group; EORTC QLQ-C30 - European Organisation for Research and Treatment of Cancer Quality of life; FACIT-F - Functional Assessment of Chronic Illness Therapy-Fatigue; IRB-approved; institutional review board approved; LAR-low anterior resection; OEE- Outcome Expectations for Exercise Scale; PROMIS- patient reported outcome measures

Physical activity interventions for disease-related physical and mental health during and following treatment in people with non- 
information system, PROMIS PF - patient reported outcome measures information system physical function; PROMIS PI-patient reported outcome measures information system pain interference; PROMS- patient reported outcome measures; PSEFSM- perceived self-efficacy for fatigue self-management TNM- classification of malignant tumours; Tx- treatment QoL: quality of life; RCT: randomised controlled trial; UK: United Kingdom; USA: United States of America

\section{DATA AND ANALYSES}

Comparison 1. Physical activity versus usual care for physical function

\begin{tabular}{lllll}
\hline Outcome or subgroup title & $\begin{array}{l}\text { No. of } \\
\text { studies }\end{array}$ & $\begin{array}{l}\text { No. of } \\
\text { partici- } \\
\text { pants }\end{array}$ & Statistical method & Effect size \\
\hline $\begin{array}{l}1.1 \text { Objective measures more than 12 weeks to 6 } \\
\text { months follow-up (30-Second Chair Stand Test) }\end{array}$ & 2 & 39 & $\begin{array}{l}\text { Mean Difference (IV, Fixed, 95\% } \\
\text { Cl) }\end{array}$ & $\begin{array}{l}0.76[-1.84, \\
3.36]\end{array}$ \\
\hline $\begin{array}{l}1.2 \text { Subjective measures more than 12 weeks to 6 } \\
\text { months follow-up }\end{array}$ & 3 & 156 & $\begin{array}{l}\text { Std. Mean Difference (IV, Ran- } \\
\text { dom, 95\% Cl) }\end{array}$ & $\begin{array}{l}0.09[-0.24, \\
0.42]\end{array}$ \\
\hline
\end{tabular}

Analysis 1.1. Comparison 1: Physical activity versus usual care for physical function, Outcome 1: Objective measures more than 12 weeks to 6 months follow-up (30-Second Chair Stand Test)

\begin{tabular}{|c|c|c|c|c|c|c|c|c|c|}
\hline \multirow[b]{2}{*}{ Study or Subgroup } & \multicolumn{3}{|c|}{ Physical activity } & \multicolumn{3}{|c|}{ Usual care } & \multicolumn{2}{|r|}{ Mean Difference } & Mean Difference \\
\hline & Mean & SD & Total & Mean & SD & Total & Weight & IV, Fixed, $95 \%$ CI & IV, Fixed, $95 \%$ CI \\
\hline
\end{tabular}

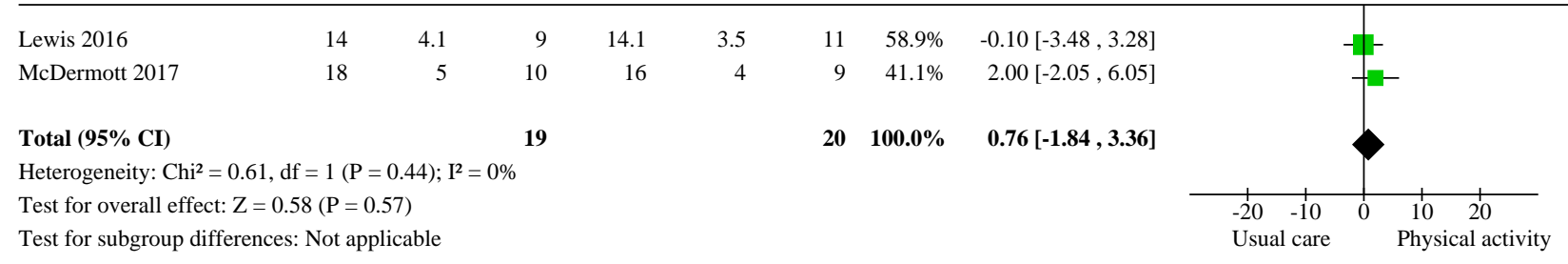

\section{Analysis 1.2. Comparison 1: Physical activity versus usual care for physical function, Outcome 2: Subjective measures more than 12 weeks to 6 months follow-up}

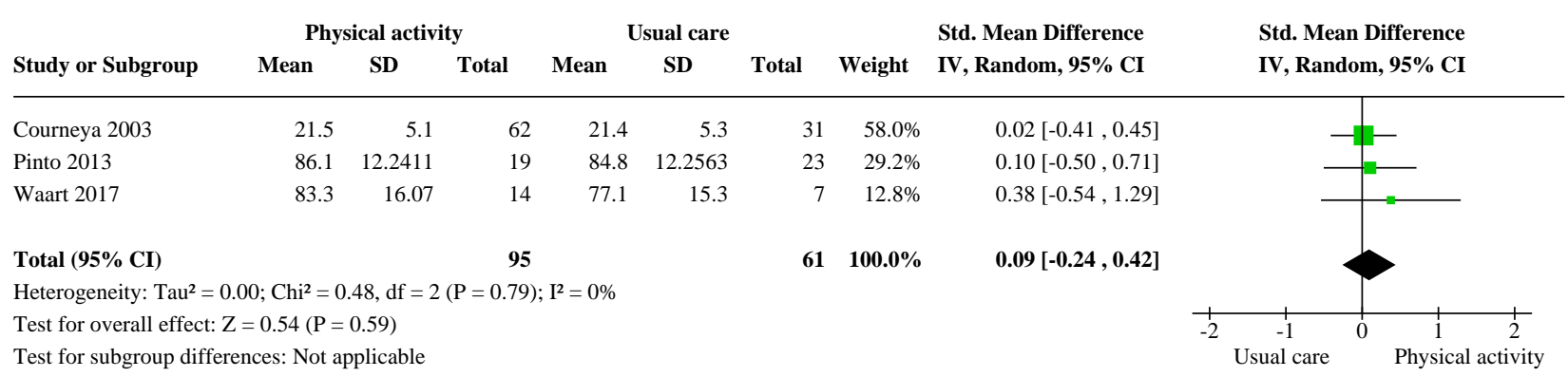


Comparison 2. Physical activity versus usual care for disease-related mental health

\begin{tabular}{|c|c|c|c|c|}
\hline Outcome or subgroup title & $\begin{array}{l}\text { No. of } \\
\text { studies }\end{array}$ & $\begin{array}{l}\text { No. of } \\
\text { partici- } \\
\text { pants }\end{array}$ & Statistical method & Effect size \\
\hline $\begin{array}{l}\text { 2.1 Depression: more than } 12 \text { weeks to } 6 \text { months } \\
\text { follow-up }\end{array}$ & 4 & 198 & $\begin{array}{l}\text { Std. Mean Difference (IV, Random, } \\
95 \% \mathrm{CI} \text { ) }\end{array}$ & $\begin{array}{l}-0.21[-0.50, \\
0.08]\end{array}$ \\
\hline $\begin{array}{l}2.2 \text { Depression: more than } 6 \text { months to } 12 \\
\text { months follow-up (HADS) }\end{array}$ & 2 & 48 & Mean Difference (IV, Fixed, 95\% CI) & $\begin{array}{l}-1.20[-2.72 \\
0.31]\end{array}$ \\
\hline $\begin{array}{l}\text { 2.3 Anxiety: more than } 12 \text { weeks to } 6 \text { months fol- } \\
\text { low-up }\end{array}$ & 4 & 198 & $\begin{array}{l}\text { Std. Mean Difference (IV, Random, } \\
95 \% \mathrm{CI})\end{array}$ & $\begin{array}{l}-0.11[-0.40, \\
0.18]\end{array}$ \\
\hline $\begin{array}{l}\text { 2.4 Anxiety: more than } 6 \text { months to } 12 \text { months } \\
\text { follow-up (HADS) }\end{array}$ & 2 & 47 & Mean Difference (IV, Fixed, 95\% CI) & $\begin{array}{l}1.79[-0.37 \\
3.94]\end{array}$ \\
\hline
\end{tabular}

Analysis 2.1. Comparison 2: Physical activity versus usual care for disease-related mental health, Outcome 1: Depression: more than 12 weeks to 6 months follow-up

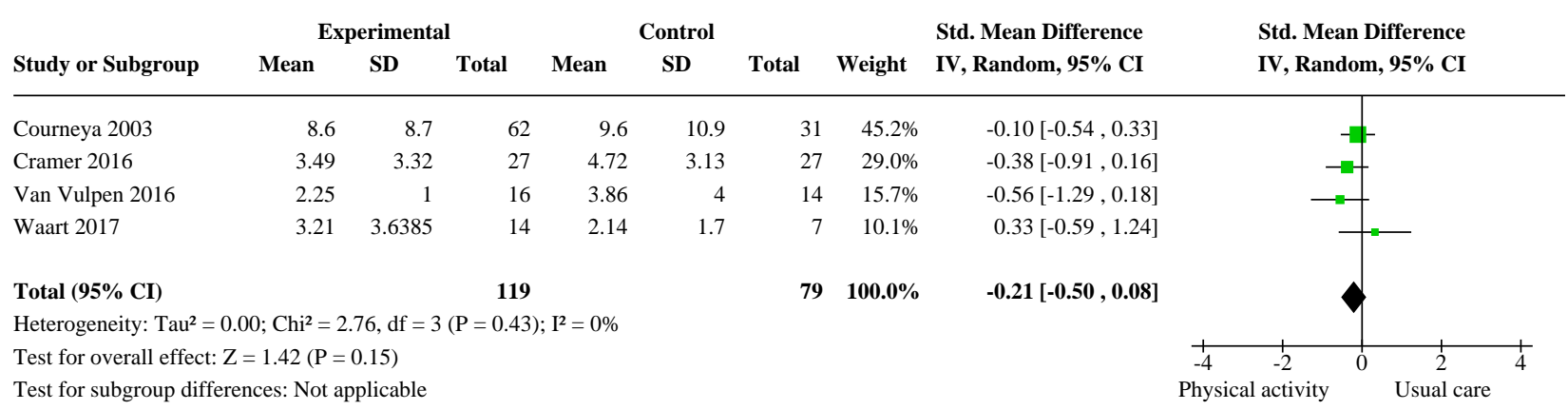

\section{Analysis 2.2. Comparison 2: Physical activity versus usual care for disease-related mental health, Outcome 2: Depression: more than 6 months to 12 months follow-up (HADS)}

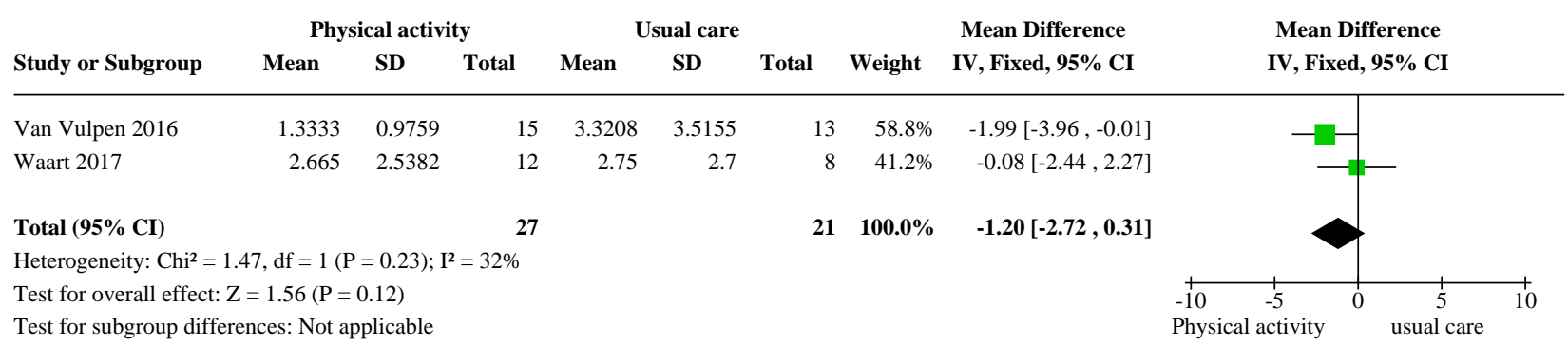


Analysis 2.3. Comparison 2: Physical activity versus usual care for disease-related mental health, Outcome 3: Anxiety: more than 12 weeks to 6 months follow-up

\begin{tabular}{|c|c|c|c|c|c|c|c|c|c|c|c|}
\hline \multirow{3}{*}{$\begin{array}{l}\text { Study or Subgroup } \\
\text { Courneya } 2003\end{array}$} & \multicolumn{3}{|c|}{ Physical activity } & \multicolumn{3}{|c|}{ usual care } & \multirow[b]{2}{*}{ Weight } & \multirow{2}{*}{$\begin{array}{l}\text { Std. Mean Difference } \\
\text { IV, Random, } 95 \% \text { CI }\end{array}$} & \multirow{2}{*}{\multicolumn{3}{|c|}{$\begin{array}{l}\text { Std. Mean Difference } \\
\text { IV, Random, } 95 \% \text { CI }\end{array}$}} \\
\hline & Mean & SD & Total & Mean & SD & Total & & & & & \\
\hline & 33.5 & 11.5 & 62 & 35.5 & 12.6 & 31 & $44.8 \%$ & $-0.17[-0.60,0.26]$ & & L & \\
\hline Cramer 2016 & 5.28 & 2.15 & 27 & 5.86 & 2.48 & 27 & $29.1 \%$ & $-0.25[-0.78,0.29]$ & & & \\
\hline Van Vulpen 2016 & 2.9 & 2 & 16 & 3.1 & 3 & 14 & $16.2 \%$ & $-0.08[-0.79,0.64]$ & & & \\
\hline Waart 2017 & 3.93 & 4.3289 & 14 & 2 & 2 & 7 & $9.8 \%$ & $0.49[-0.43,1.42]$ & & & \\
\hline Total $(95 \%$ CI $)$ & & & 119 & & & 79 & $100.0 \%$ & $-0.11[-0.40,0.18]$ & & & \\
\hline \multicolumn{12}{|c|}{ Heterogeneity: $\mathrm{Tau}^{2}=0.00 ; \mathrm{Chi}^{2}=1.97, \mathrm{df}=3(\mathrm{P}=0.58) ; \mathrm{I}^{2}=0 \%$} \\
\hline \multicolumn{9}{|c|}{ Test for overall effect: $\mathrm{Z}=0.75(\mathrm{P}=0.45)$} & $\frac{1}{-2}$ & $\begin{array}{c}1 \\
-1\end{array}$ & $\frac{4}{2}$ \\
\hline \multicolumn{9}{|c|}{ Test for subgroup differences: Not applicable } & Physi & al activity & \\
\hline
\end{tabular}

Analysis 2.4. Comparison 2: Physical activity versus usual care for disease-related mental health, Outcome 4: Anxiety: more than 6 months to 12 months follow-up (HADS)

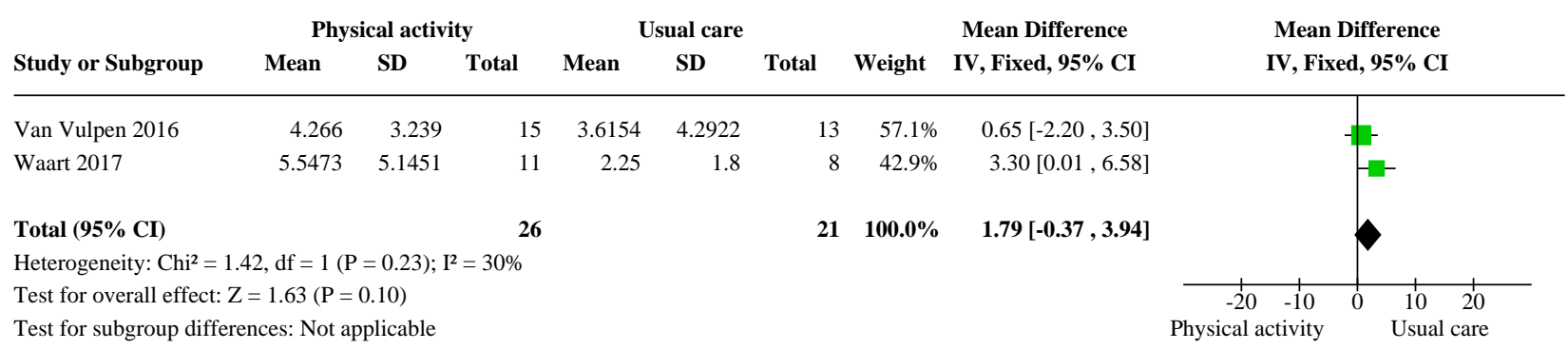

\section{Comparison 3. Physical activity versus usual care for physical fitness (aerobic fitness)}

\begin{tabular}{|c|c|c|c|c|}
\hline Outcome or subgroup title & $\begin{array}{l}\text { No. of } \\
\text { studies }\end{array}$ & $\begin{array}{l}\text { No. of } \\
\text { partici- } \\
\text { pants }\end{array}$ & Statistical method & Effect size \\
\hline 3.1 Up to 12 weeks follow-up & 7 & 295 & Std. Mean Difference (IV, Random, 95\% CI) & $0.82[0.34,1.29]$ \\
\hline $\begin{array}{l}3.2 \text { Change from baseline up to } 12 \\
\text { weeks follow-up }\end{array}$ & 3 & 81 & Std. Mean Difference (IV, Random, 95\% CI) & $0.89[0.43,1.36]$ \\
\hline $\begin{array}{l}\text { 3.3 More than } 12 \text { weeks to } 6 \text { months } \\
\text { follow-up }\end{array}$ & 7 & 248 & Std. Mean Difference (IV, Random, 95\% CI) & $0.56[0.29,0.82]$ \\
\hline $\begin{array}{l}3.4 \text { Change from baseline more than } 12 \\
\text { weeks to } 6 \text { months follow-up }\end{array}$ & 2 & 51 & Std. Mean Difference (IV, Fixed, 95\% CI) & $0.62[0.05,1.19]$ \\
\hline $\begin{array}{l}3.5 \text { More than } 6 \text { months to } 12 \text { months } \\
\text { follow-up }\end{array}$ & 4 & 272 & Std. Mean Difference (IV, Random, 95\% CI) & $0.44[-0.04,0.92]$ \\
\hline
\end{tabular}


Analysis 3.1. Comparison 3: Physical activity versus usual care for physical fitness (aerobic fitness), Outcome 1: Up to 12 weeks follow-up

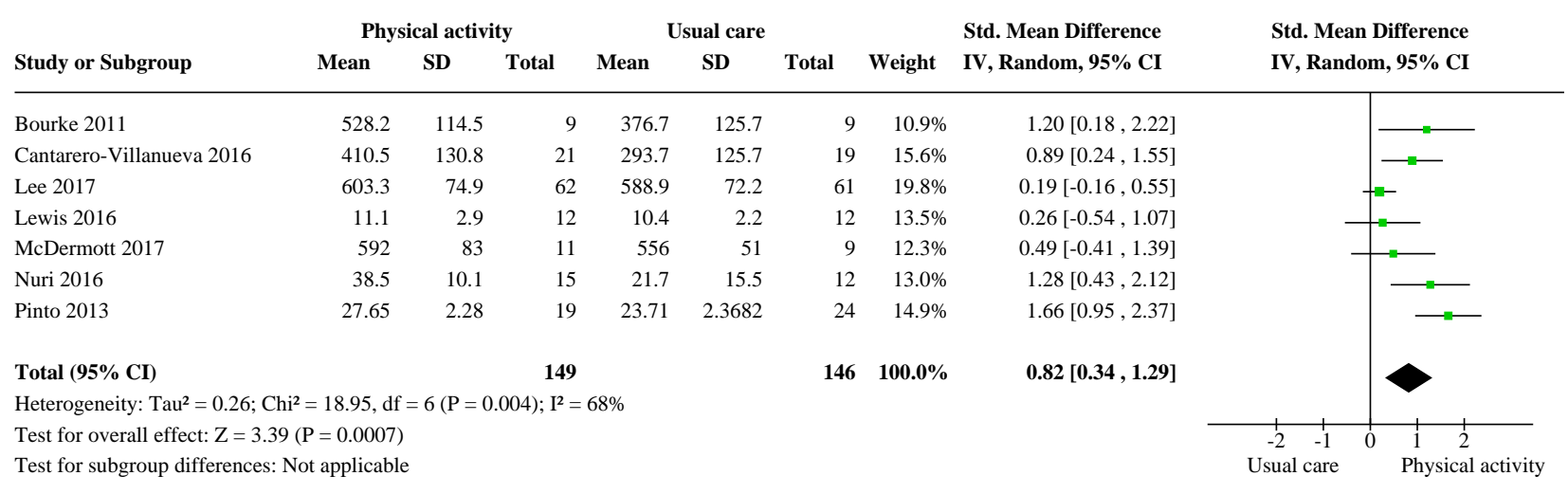

Analysis 3.2. Comparison 3: Physical activity versus usual care for physical fitness (aerobic fitness), Outcome 2: Change from baseline up to 12 weeks follow-up

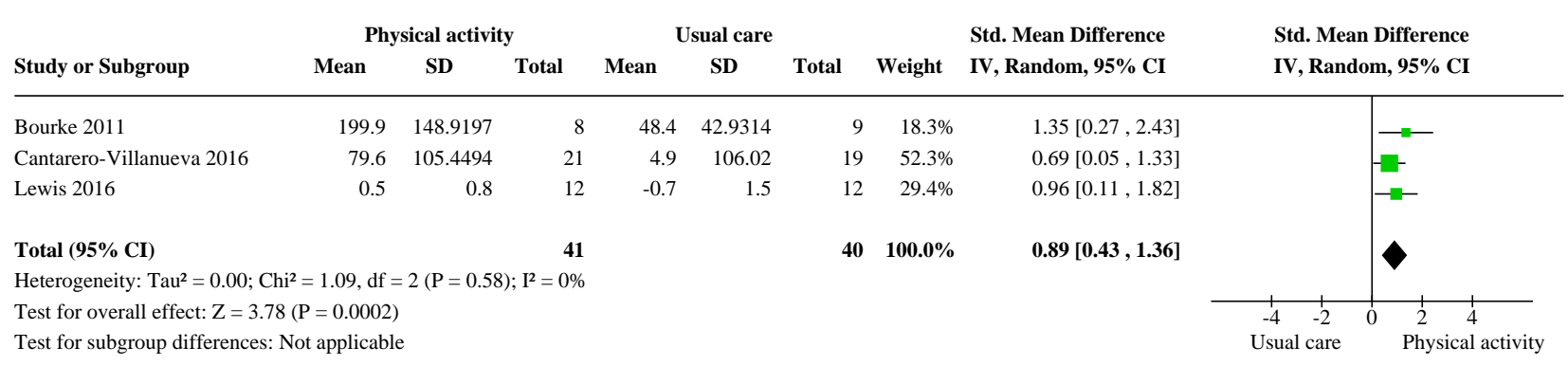

Analysis 3.3. Comparison 3: Physical activity versus usual care for physical fitness (aerobic fitness), Outcome 3: More than 12 weeks to 6 months follow-up

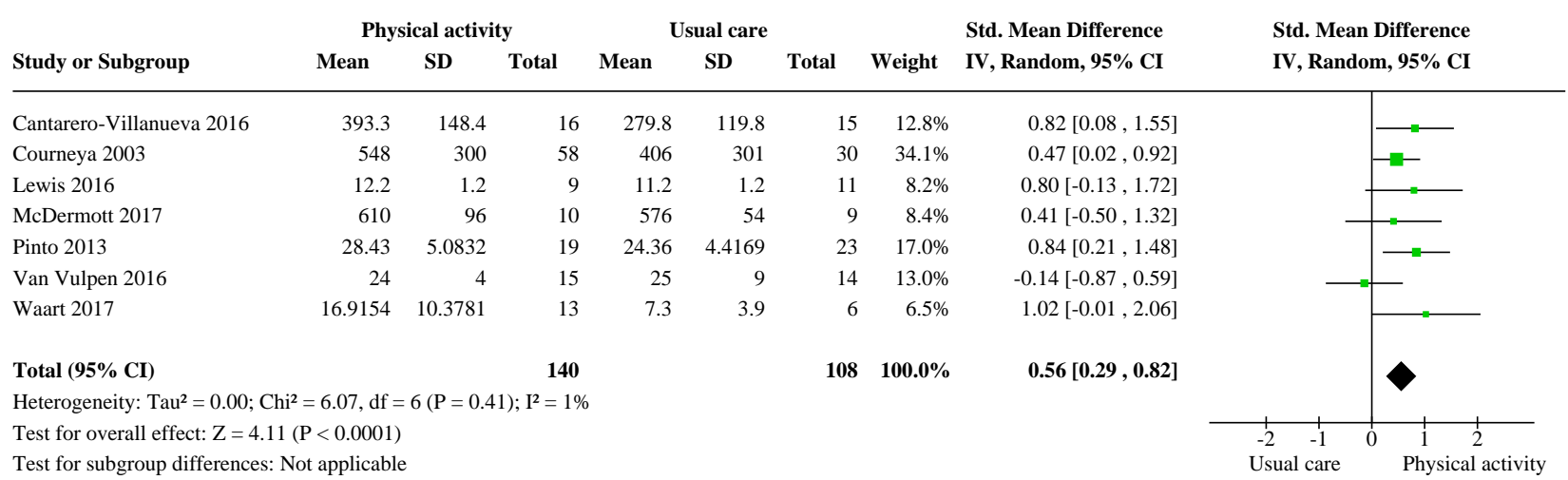


Analysis 3.4. Comparison 3: Physical activity versus usual care for physical fitness (aerobic fitness), Outcome 4: Change from baseline more than 12 weeks to 6 months follow-up

\begin{tabular}{|c|c|c|c|c|c|c|c|c|c|c|}
\hline \multirow[b]{2}{*}{ Study or Subgroup } & \multicolumn{3}{|c|}{ Physical activity } & \multicolumn{3}{|c|}{ Usual care } & \multicolumn{2}{|r|}{ Std. Mean Difference } & \multirow{2}{*}{\multicolumn{2}{|c|}{$\begin{array}{l}\text { Std. Mean Difference } \\
\text { IV, Fixed, 95\% CI }\end{array}$}} \\
\hline & Mean & SD & Total & Mean & SD & Total & Weight & IV, Fixed, 95\% CI & & \\
\hline Cantarero-Villanueva 2016 & 62.4 & 85.3879 & 16 & -8.9 & 86.4962 & 15 & $59.3 \%$ & $0.81[0.07,1.54]$ & & _ \\
\hline Lewis 2016 & 2 & 3.1 & 9 & 1.1 & 1.8 & 11 & $40.7 \%$ & $0.35[-0.54,1.24]$ & & - \\
\hline Total $(95 \% \mathrm{CI})$ & & & 25 & & & 26 & $100.0 \%$ & $0.62[0.05,1.19]$ & & \\
\hline \multicolumn{11}{|c|}{ Heterogeneity: $\mathrm{Chi}^{2}=0.61, \mathrm{df}=1(\mathrm{P}=0.44) ; \mathrm{I}^{2}=0 \%$} \\
\hline \multicolumn{9}{|c|}{ Test for overall effect: $\mathrm{Z}=2.15(\mathrm{P}=0.03)$} & $\begin{array}{cc}1 & 1 \\
-2 & -1\end{array}$ & 12 \\
\hline \multicolumn{9}{|c|}{ Test for subgroup differences: Not applicable } & Usual care & Physica \\
\hline
\end{tabular}

Analysis 3.5. Comparison 3: Physical activity versus usual care for physical fitness (aerobic fitness), Outcome 5: More than 6 months to 12 months follow-up

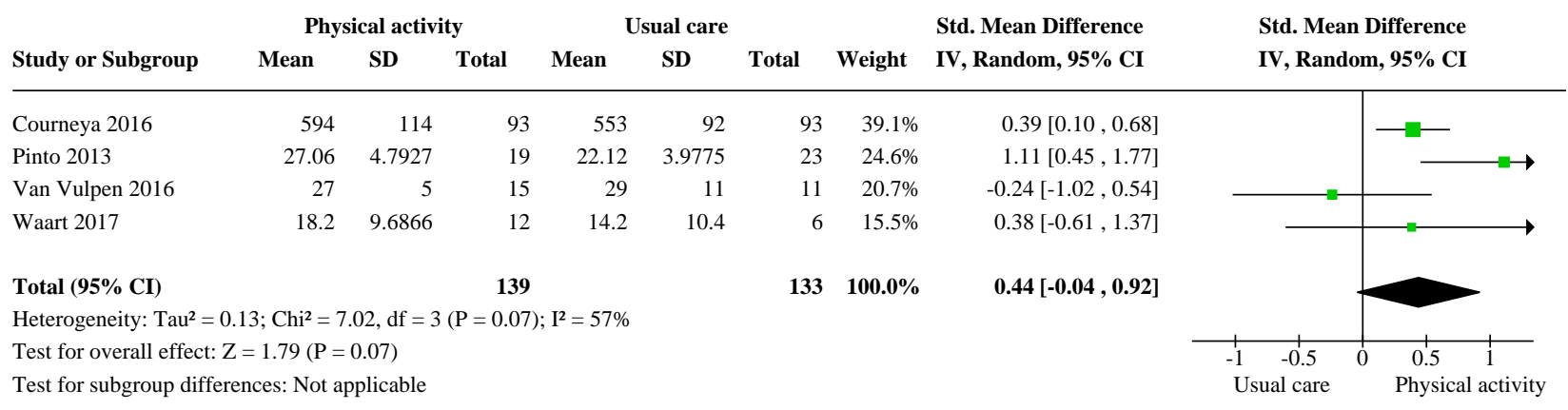

Comparison 4. Physical activity versus usual care for physical fitness (hand grip strength)

\begin{tabular}{llllll}
\hline Outcome or subgroup title & $\begin{array}{l}\text { No. of } \\
\text { studies }\end{array}$ & $\begin{array}{l}\text { No. of } \\
\text { partici- } \\
\text { pants }\end{array}$ & Statistical method & Effect size \\
\hline $\begin{array}{l}4.1 \text { Up to } 12 \text { weeks follow-up (hand dynamome- } \\
\text { try) }\end{array}$ & 2 & 147 & $\begin{array}{l}\text { Mean Difference (IV, Fixed, 95\% } \\
\text { Cl) }\end{array}$ & $\begin{array}{l}1.92[-1.17, \\
5.00]\end{array}$ \\
\hline $\begin{array}{l}\text { 4.2 More than 12 weeks to 6 months follow-up } \\
\text { (hand dynamometry) }\end{array}$ & 2 & 39 & $\begin{array}{l}\text { Mean Difference (IV, Fixed, 95\% } \\
\text { Cl) }\end{array}$ & 0.94 [-5.98, \\
\hline
\end{tabular}

Analysis 4.1. Comparison 4: Physical activity versus usual care for physical fitness (hand grip strength), Outcome 1: Up to 12 weeks follow-up (hand dynamometry)

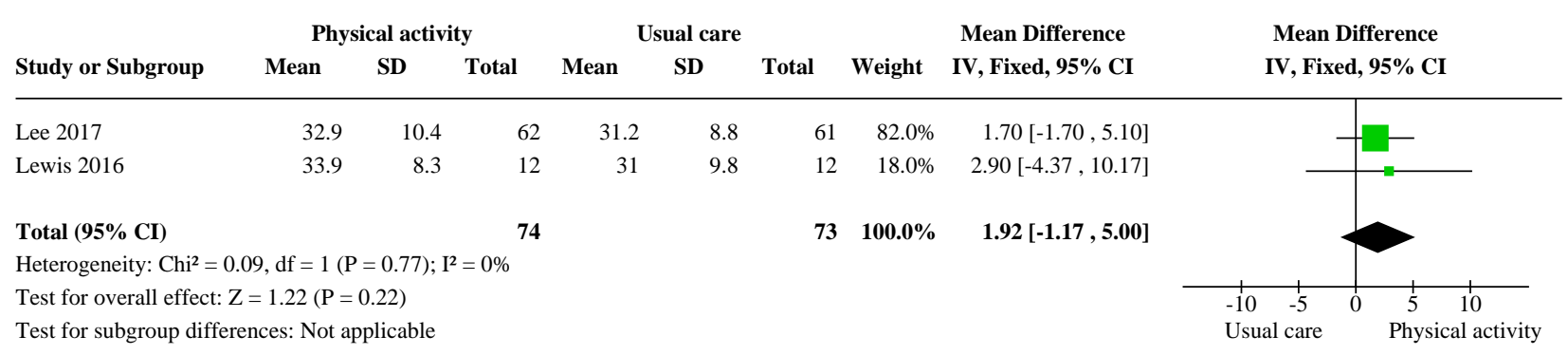


Analysis 4.2. Comparison 4: Physical activity versus usual care for physical fitness (hand grip strength), Outcome 2: More than 12 weeks to 6 months follow-up (hand dynamometry)

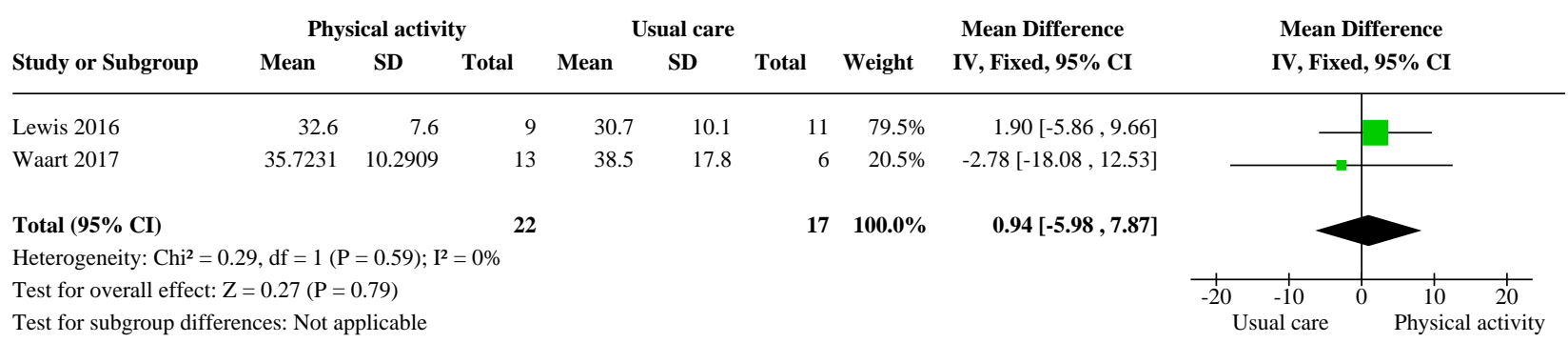

Comparison 5. Physical activity versus usual care for physical fitness (flexibility)

\begin{tabular}{lllll}
\hline Outcome or subgroup title & $\begin{array}{l}\text { No. of } \\
\text { studies }\end{array}$ & $\begin{array}{l}\text { No. of par- } \\
\text { ticipants }\end{array}$ & Statistical method & Effect size \\
\hline $\begin{array}{l}\text { 5.1 More than 12 weeks to 6 months fol- } \\
\text { low-up }\end{array}$ & 2 & 119 & $\begin{array}{l}\text { Std. Mean Difference (IV, Fixed, 95\% } \\
\text { CI) }\end{array}$ & $0.02[-0.36,0.39]$ \\
\hline
\end{tabular}

Analysis 5.1. Comparison 5: Physical activity versus usual care for physical fitness (flexibility), Outcome 1: More than 12 weeks to 6 months follow-up

\begin{tabular}{|c|c|c|c|c|c|c|c|c|c|}
\hline \multirow[b]{2}{*}{ Study or Subgroup } & \multicolumn{2}{|c|}{ Usual care } & \multicolumn{4}{|c|}{ Physical activity } & \multirow[b]{2}{*}{ Weight } & \multirow{2}{*}{$\begin{array}{l}\text { Std. Mean Difference } \\
\text { IV, Fixed, 95\% CI }\end{array}$} & \multirow{2}{*}{$\begin{array}{l}\text { Std. Mean Difference } \\
\text { IV, Fixed, 95\% CI }\end{array}$} \\
\hline & Mean & SD & Total & Mean & SD & Total & & & \\
\hline Cantarero-Villanueva 2016 & 11.1 & 12.8 & 15 & 7.7 & 10.3 & 16 & $27.9 \%$ & $0.29[-0.42,0.99]$ & \\
\hline Courneya 2003 & 20.9 & 9.6 & 58 & 21.8 & 10.9 & 30 & $72.1 \%$ & $-0.09[-0.53,0.35]$ & \\
\hline Total $(95 \%$ CI $)$ & & & 73 & & & 46 & $100.0 \%$ & $0.02[-0.36,0.39]$ & \\
\hline \multicolumn{10}{|c|}{ Heterogeneity: $\mathrm{Chi}^{2}=0.77, \mathrm{df}=1(\mathrm{P}=0.38) ; \mathrm{I}^{2}=0 \%$} \\
\hline \multirow{2}{*}{\multicolumn{4}{|c|}{$\begin{array}{l}\text { Test for overall effect: } \mathrm{Z}=0.08(\mathrm{P}=0.93) \\
\text { Test for suboroun differences: Not applicable }\end{array}$}} & & & & & & $-1 \quad-0.5$ \\
\hline & & & & & & & & & Usual care \\
\hline
\end{tabular}

Comparison 6. Physical activity versus usual care for cancer-related fatigue

\begin{tabular}{|c|c|c|c|c|}
\hline Outcome or subgroup title & $\begin{array}{l}\text { No. of } \\
\text { studies }\end{array}$ & $\begin{array}{l}\text { No. of } \\
\text { partici- } \\
\text { pants }\end{array}$ & Statistical method & Effect size \\
\hline $\begin{array}{l}6.1 \text { Up to } 12 \text { weeks follow-up (FACT-F and } \\
\text { FACIT-F) }\end{array}$ & 6 & 230 & Mean Difference (IV, Random, 95\% Cl) & $2.16[0.18,4.15]$ \\
\hline $\begin{array}{l}\text { 6.2 Change from baseline up to } 12 \text { weeks fol- } \\
\text { low-up (FACT-F and FACIT-F) }\end{array}$ & 3 & 113 & Mean Difference (IV, Fixed, 95\% CI) & $\begin{array}{l}0.41[-1.33 \\
2.14]\end{array}$ \\
\hline 6.3 More than 12 weeks to 6 months follow-up & 7 & 277 & $\begin{array}{l}\text { Std. Mean Difference (IV, Random, } \\
95 \% \mathrm{CI} \text { ) }\end{array}$ & $0.34[0.08,0.60]$ \\
\hline $\begin{array}{l}\text { 6.4 More than } 6 \text { months to } 12 \text { months fol- } \\
\text { low-up }\end{array}$ & 3 & 91 & $\begin{array}{l}\text { Std. Mean Difference (IV, Fixed, 95\% } \\
\mathrm{CI} \text { ) }\end{array}$ & $\begin{array}{l}0.25[-0.16 \\
0.67]\end{array}$ \\
\hline
\end{tabular}


Analysis 6.1. Comparison 6: Physical activity versus usual care for cancerrelated fatigue, Outcome 1: Up to 12 weeks follow-up (FACT-F and FACIT-F)

\begin{tabular}{|c|c|c|c|c|c|c|c|c|c|}
\hline \multirow[b]{2}{*}{ Study or Subgroup } & \multicolumn{3}{|c|}{ Physical activity } & \multicolumn{3}{|c|}{ Usual care } & \multicolumn{2}{|r|}{ Mean Difference } & Mean Difference \\
\hline & Mean & SD & Total & Mean & SD & Total & Weight & IV, Random, $95 \%$ CI & IV, Random, $95 \%$ CI \\
\hline
\end{tabular}

\begin{tabular}{lrrrrrrrr}
\hline Bourke 2011 & 48 & 4 & 9 & 43 & 6 & 9 & $15.0 \%$ & $5.00[0.29,9.71]$ \\
Cramer 2016 & 43.02 & 6.52 & 27 & 40.45 & 8.04 & 27 & $20.3 \%$ & $2.57[-1.33,6.47]$ \\
Kim 2018 & 42.6 & 8.5 & 37 & 42.3 & 7.5 & 34 & $21.9 \%$ & $0.30[-3.42,4.02]$ \\
Lewis 2016 & 46.2 & 5.8 & 12 & 44 & 6.7 & 12 & $13.5 \%$ & $2.20[-2.81,7.21]$ \\
McDermott 2017 & 46.4 & 6.2 & 11 & 37.1 & 12.1 & 9 & $4.9 \%$ & $9.30[0.59,18.01]$ \\
Pinto 2013 & 42.2 & 5.8093 & 19 & 41.9 & 5.6837 & 24 & $24.4 \%$ & $0.30[-3.16,3.76]$
\end{tabular}

Total (95\% CI) 115

$115 \quad 100.0 \%$

$2.16[0.18,4.15]$

Heterogeneity: $\mathrm{Tau}^{2}=1.09 ; \mathrm{Chi}^{2}=6.07, \mathrm{df}=5(\mathrm{P}=0.30) ; \mathrm{I}^{2}=18 \%$

Test for overall effect: $\mathrm{Z}=2.14(\mathrm{P}=0.03)$

Test for subgroup differences: Not applicable

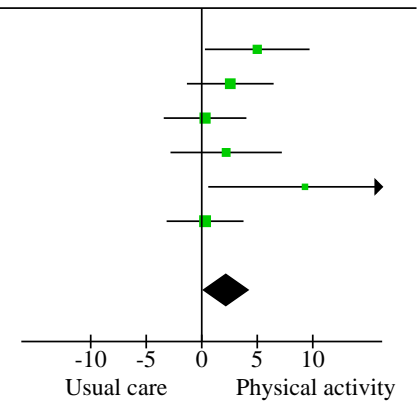

Analysis 6.2. Comparison 6: Physical activity versus usual care for cancer-related fatigue, Outcome 2: Change from baseline up to 12 weeks follow-up (FACT-F and FACIT-F)

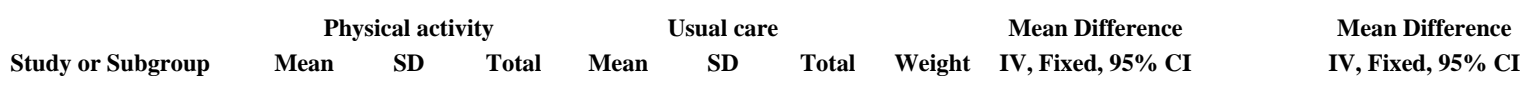

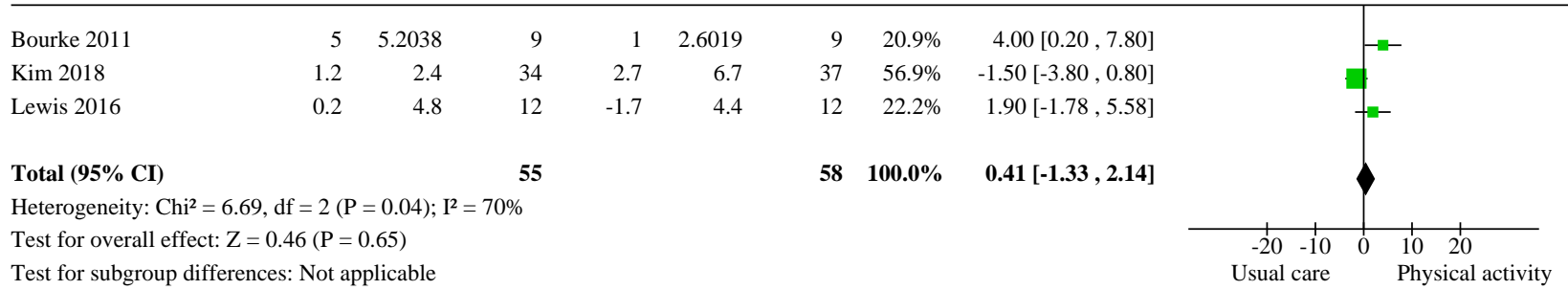

Analysis 6.3. Comparison 6: Physical activity versus usual care for cancerrelated fatigue, Outcome 3: More than 12 weeks to 6 months follow-up

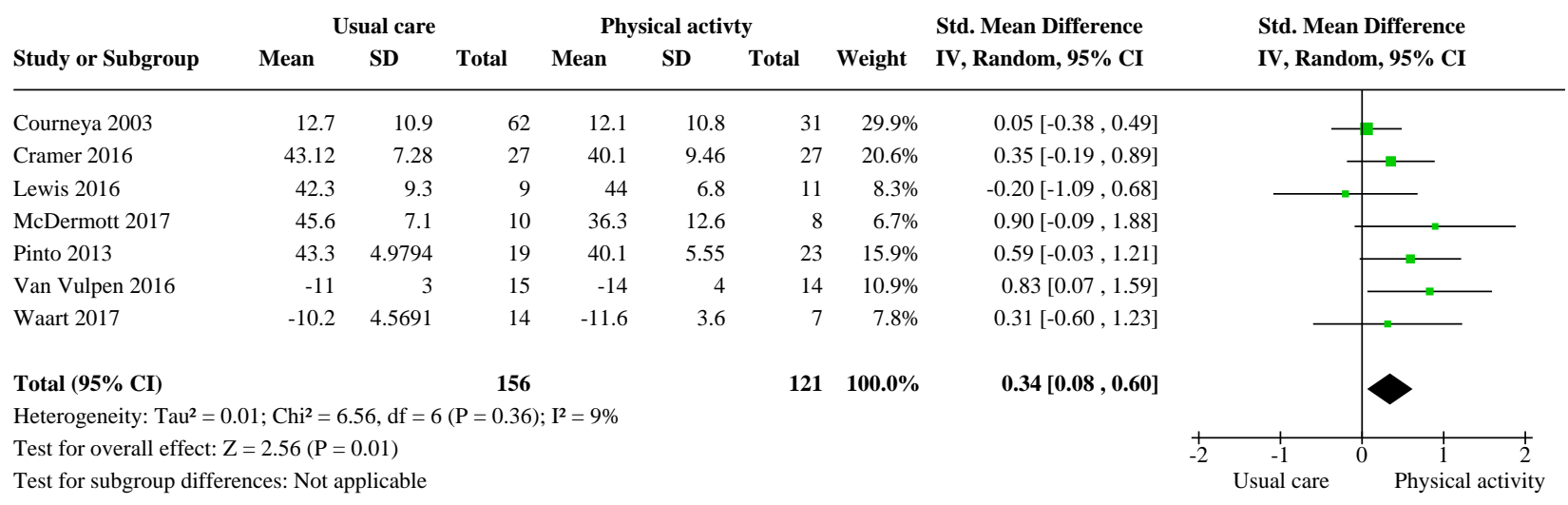


Analysis 6.4. Comparison 6: Physical activity versus usual care for cancerrelated fatigue, Outcome 4: More than 6 months to 12 months follow-up

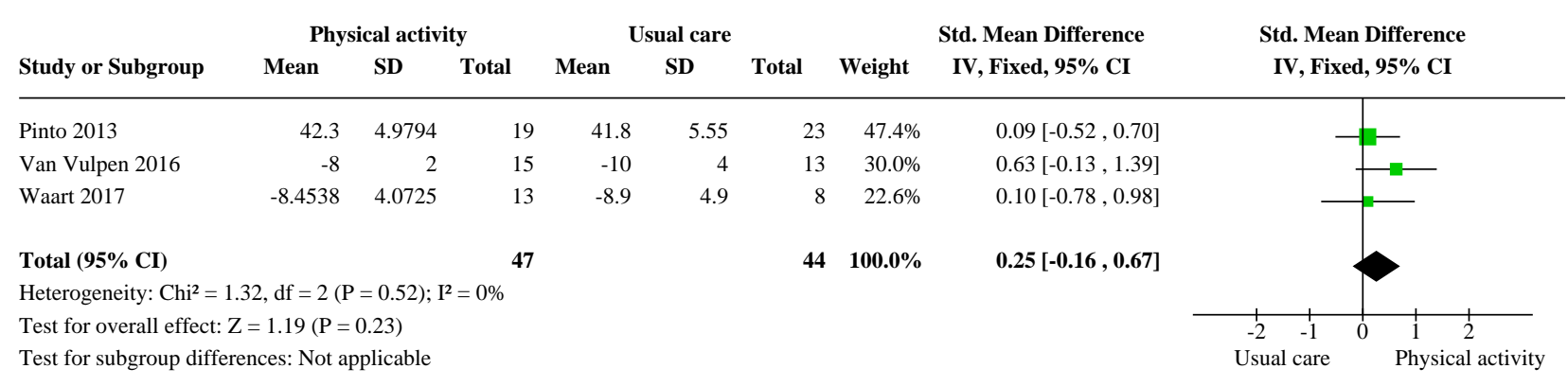

Comparison 7. Physical activity versus usual care for anthropometric measure of weight (kg)

\begin{tabular}{|c|c|c|c|c|}
\hline Outcome or subgroup title & $\begin{array}{l}\text { No. of } \\
\text { studies }\end{array}$ & $\begin{array}{l}\text { No. of } \\
\text { partici- } \\
\text { pants }\end{array}$ & Statistical method & Effect size \\
\hline 7.1 Up to 12 weeks follow-up & 6 & 252 & Mean Difference (IV, Random, 95\% CI) & $0.29[-2.55,3.14]$ \\
\hline $\begin{array}{l}7.2 \text { Change from baseline up to } 12 \text { weeks } \\
\text { follow-up }\end{array}$ & 3 & 82 & Mean Difference (IV, Random, 95\% CI) & $-1.71[-2.90,-0.51]$ \\
\hline $\begin{array}{l}7.3 \text { Change from baseline more than } 12 \\
\text { weeks to } 6 \text { months follow-up }\end{array}$ & 3 & 89 & Mean Difference (IV, Random, 95\% CI) & $-0.73[-2.17,0.72]$ \\
\hline $\begin{array}{l}\text { 7.4 More than } 12 \text { weeks to } 6 \text { months fol- } \\
\text { low-up }\end{array}$ & 3 & 74 & Mean Difference (IV, Random, 95\% CI) & $0.59[-6.87,8.04]$ \\
\hline
\end{tabular}

Analysis 7.1. Comparison 7: Physical activity versus usual care for anthropometric measure of weight $(\mathrm{kg})$, Outcome 1: Up to 12 weeks follow-up

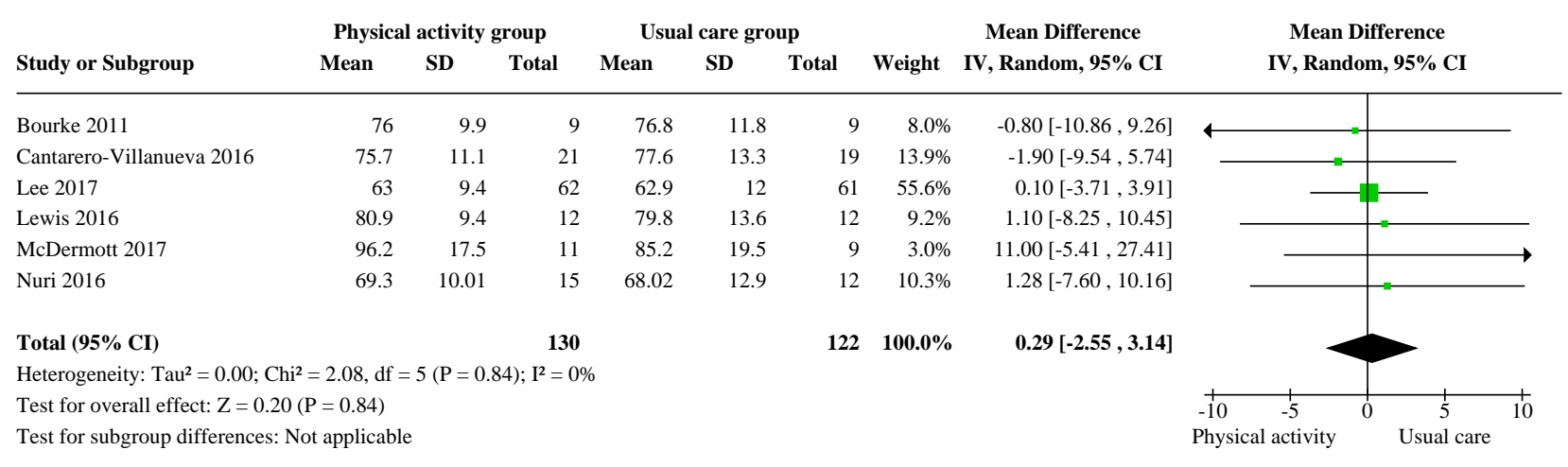


Analysis 7.2. Comparison 7: Physical activity versus usual care for anthropometric measure of weight (kg), Outcome 2: Change from baseline up to 12 weeks follow-up

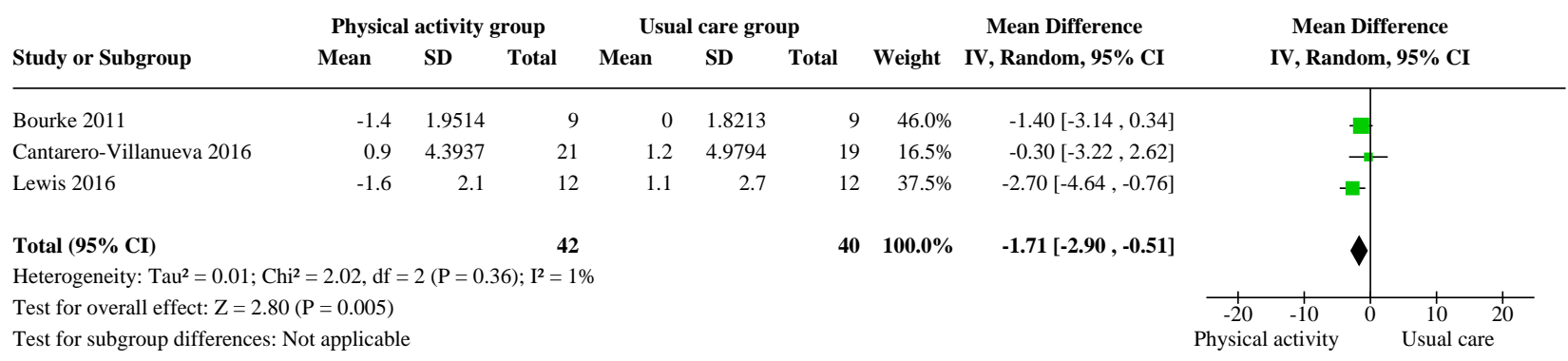

Analysis 7.3. Comparison 7: Physical activity versus usual care for anthropometric measure of weight (kg), Outcome 3: Change from baseline more than 12 weeks to 6 months follow-up

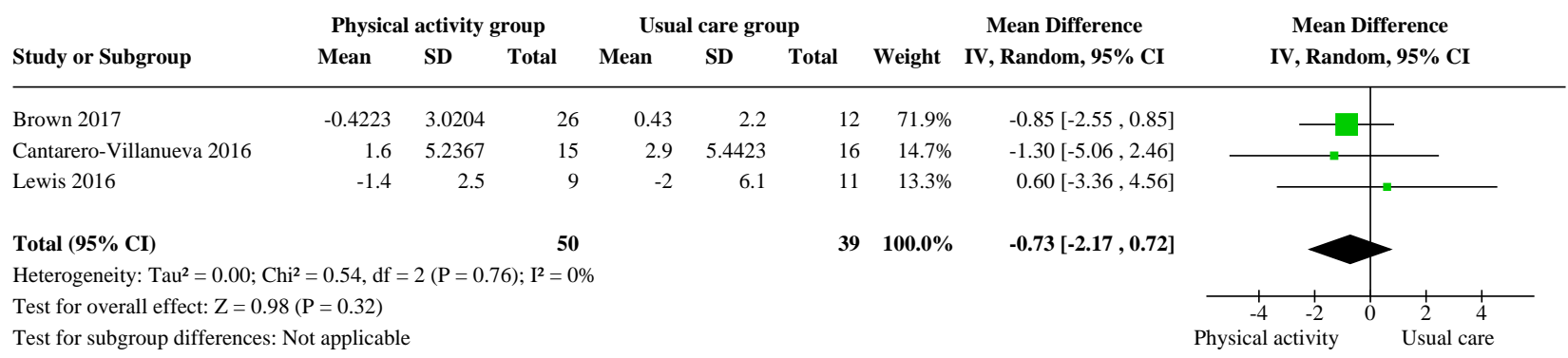

\section{Analysis 7.4. Comparison 7: Physical activity versus usual care for anthropometric measure of weight (kg), Outcome 4: More than 12 weeks to 6 months follow-up}

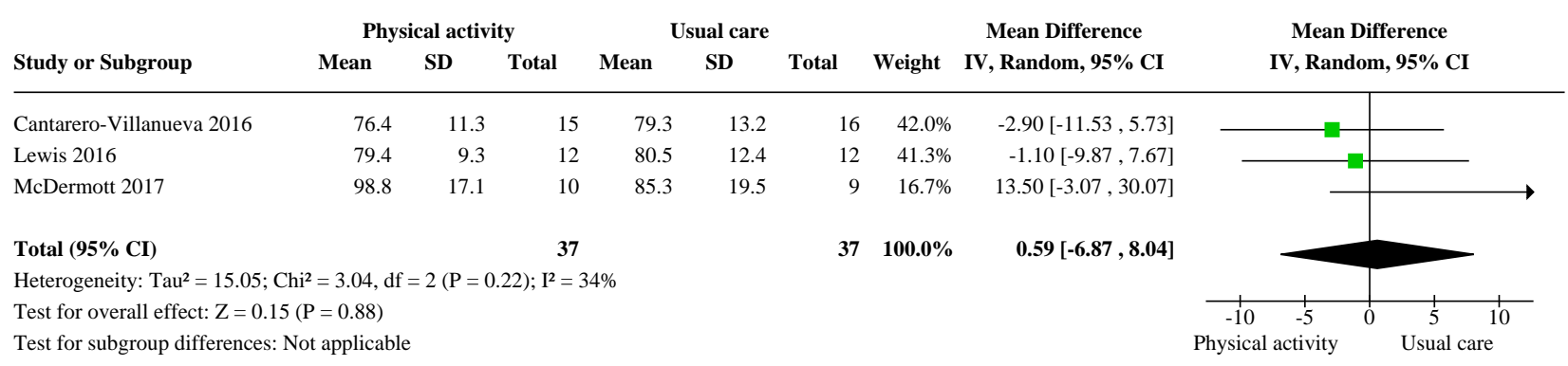

Comparison 8. Physical activity versus usual care for anthropometric measure of waist circumference

\begin{tabular}{llllll}
\hline Outcome or subgroup title & $\begin{array}{l}\text { No. of } \\
\text { studies }\end{array}$ & $\begin{array}{l}\text { No. of } \\
\text { partici- } \\
\text { pants }\end{array}$ & Statistical method & Effect size \\
\hline 8.1 Up to 12 weeks follow-up & 3 & 183 & $\begin{array}{l}\text { Mean Difference (IV, Random, 95\% } \\
\text { Cl) }\end{array}$ & $0.02[-2.88,2.93]$ \\
\hline $\begin{array}{l}\text { 8.2 More than } 12 \text { weeks to 6 months fol- } \\
\text { low-up }\end{array}$ & 2 & 50 & Mean Difference (IV, Fixed, 95\% Cl) & $1.58[-5.58,8.74]$ \\
\hline $\begin{array}{l}\text { 8.3 Change from baseline more than 12 } \\
\text { weeks to 6 months follow-up }\end{array}$ & 2 & 70 & Mean Difference (IV, Fixed, 95\% Cl) & $-2.79[-5.21,-0.36]$ \\
\hline \hline
\end{tabular}

Physical activity interventions for disease-related physical and mental health during and following treatment in people with non- 
Analysis 8.1. Comparison 8: Physical activity versus usual care for anthropometric measure of waist circumference, Outcome 1: Up to 12 weeks follow-up

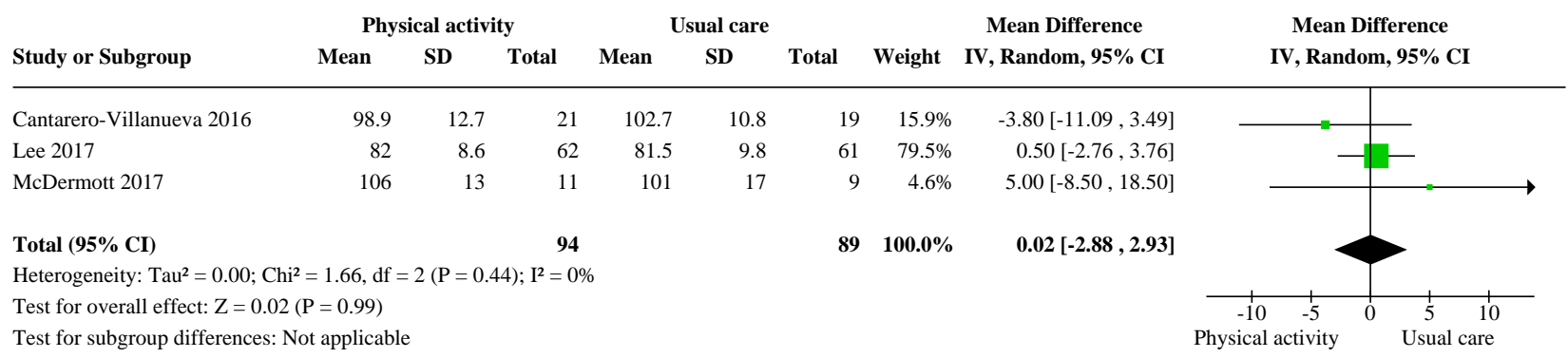

Analysis 8.2. Comparison 8: Physical activity versus usual care for anthropometric measure of waist circumference, Outcome 2: More than 12 weeks to 6 months follow-up

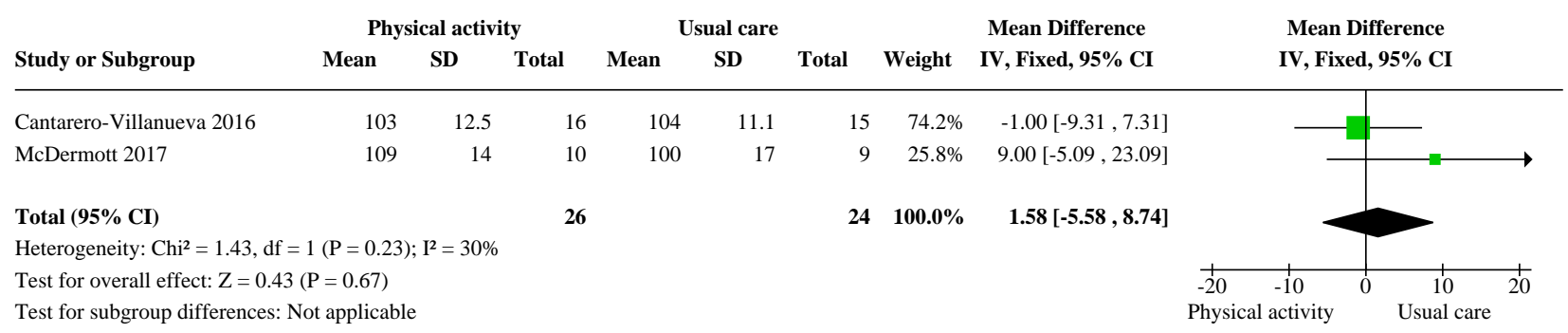

Analysis 8.3. Comparison 8: Physical activity versus usual care for anthropometric measure of waist circumference, Outcome 3: Change from baseline more than 12 weeks to 6 months follow-up

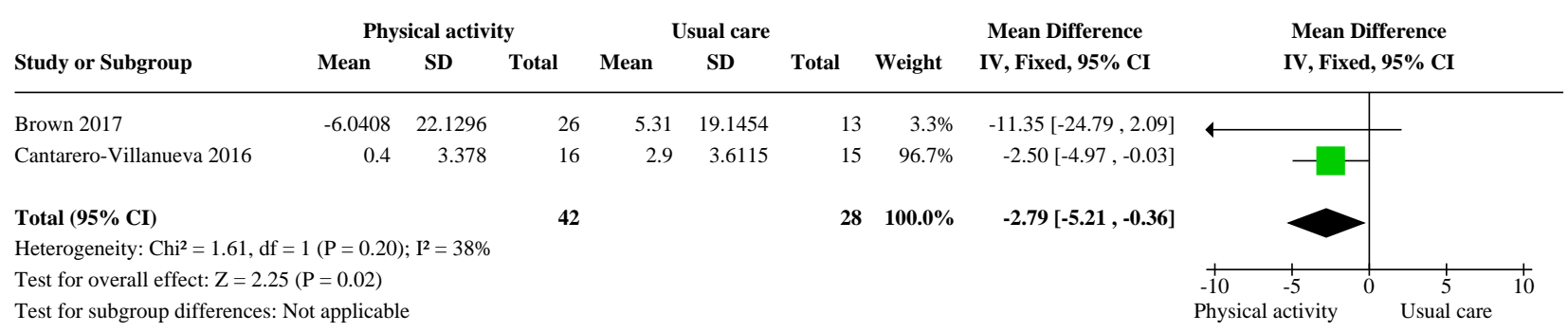

Comparison 9. Physical activity versus usual care anthropometric measure of waist to hip ratio

\begin{tabular}{llllll}
\hline Outcome or subgroup title & $\begin{array}{l}\text { No. of } \\
\text { studies }\end{array}$ & $\begin{array}{l}\text { No. of } \\
\text { partici- } \\
\text { pants }\end{array}$ & Statistical method & Effect size \\
\hline 9.1 Up to 12 weeks follow-up & 3 & 62 & Mean Difference (IV, Random, 95\% Cl) & $-0.01[-0.12,0.10]$ \\
\hline $\begin{array}{l}\text { 9.2 Change from baseline up to } 12 \\
\text { weeks follow-up }\end{array}$ & 2 & 42 & Mean Difference (IV, Fixed, 95\% Cl) & $-0.00[-0.02,0.02]$ \\
\hline $\begin{array}{l}\text { 9.3 More than } 12 \text { weeks to 6 months } \\
\text { follow-up }\end{array}$ & 2 & 43 & Mean Difference (IV, Fixed, 95\% Cl) & $0.06[-0.03,0.14]$ \\
\hline
\end{tabular}


Analysis 9.1. Comparison 9: Physical activity versus usual care anthropometric measure of waist to hip ratio, Outcome 1: Up to 12 weeks follow-up

\begin{tabular}{|c|c|c|c|c|c|c|c|c|c|}
\hline \multirow[b]{2}{*}{ Study or Subgroup } & \multicolumn{3}{|c|}{ Physical activity } & \multicolumn{3}{|c|}{ Usual care } & \multirow[b]{2}{*}{ Weight } & \multirow{2}{*}{$\begin{array}{c}\text { Mean Difference } \\
\text { IV, Random, 95\% CI }\end{array}$} & \multirow{2}{*}{$\begin{array}{c}\text { Mean Difference } \\
\text { IV, Random, 95\% CI }\end{array}$} \\
\hline & Mean & SD & Total & Mean & SD & Total & & & \\
\hline Bourke 2011 & 0.83 & 0.07 & 9 & 0.94 & 0.07 & 9 & $34.6 \%$ & $-0.11[-0.17,-0.05]$ & $-1-$ \\
\hline Lewis 2016 & 0.95 & 0.1 & 12 & 0.89 & 0.1 & 12 & $32.5 \%$ & $0.06[-0.02,0.14]$ & - \\
\hline McDermott 2017 & 0.96 & 0.07 & 11 & 0.93 & 0.1 & 9 & $32.9 \%$ & $0.03[-0.05,0.11]$ & \\
\hline Total $(95 \%$ CI $)$ & & & 32 & & & 30 & $100.0 \%$ & $-0.01[-0.12,0.10]$ & \\
\hline \multicolumn{10}{|c|}{ Heterogeneity: $\mathrm{Tau}^{2}=0.01 ; \mathrm{Chi}^{2}=12.88, \mathrm{df}=2(\mathrm{P}=0.002) ; \mathrm{I}^{2}=84 \%$} \\
\hline \multicolumn{9}{|c|}{ Test for overall effect: $\mathrm{Z}=0.16(\mathrm{P}=0.87)$} & $-0.5 \quad-0.25$ \\
\hline Test for subgroup dif & ces: Not a & plicable & & & & & & & Physical activity \\
\hline
\end{tabular}

Analysis 9.2. Comparison 9: Physical activity versus usual care anthropometric measure of waist to hip ratio, Outcome 2: Change from baseline up to 12 weeks follow-up

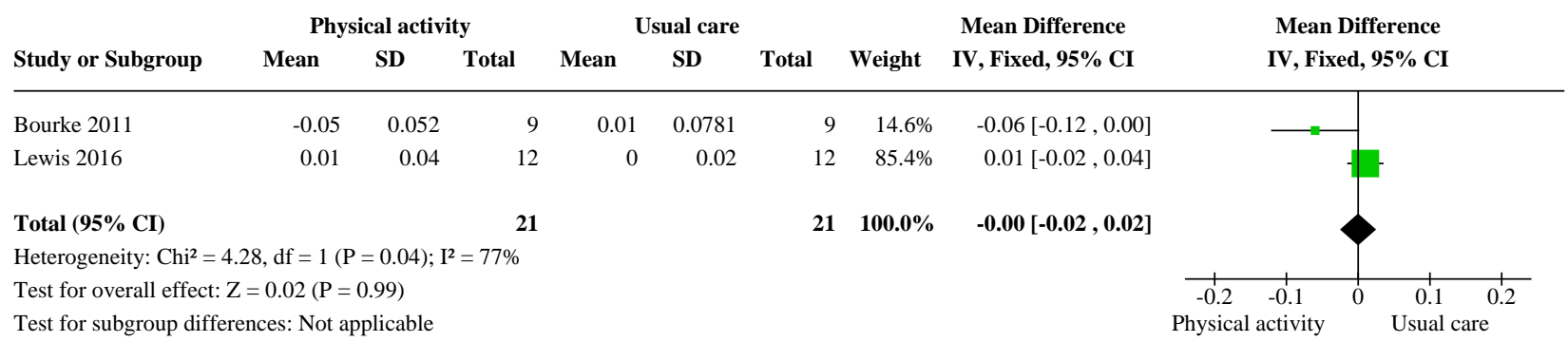

Analysis 9.3. Comparison 9: Physical activity versus usual care anthropometric measure of waist to hip ratio, Outcome 3: More than 12 weeks to 6 months follow-up

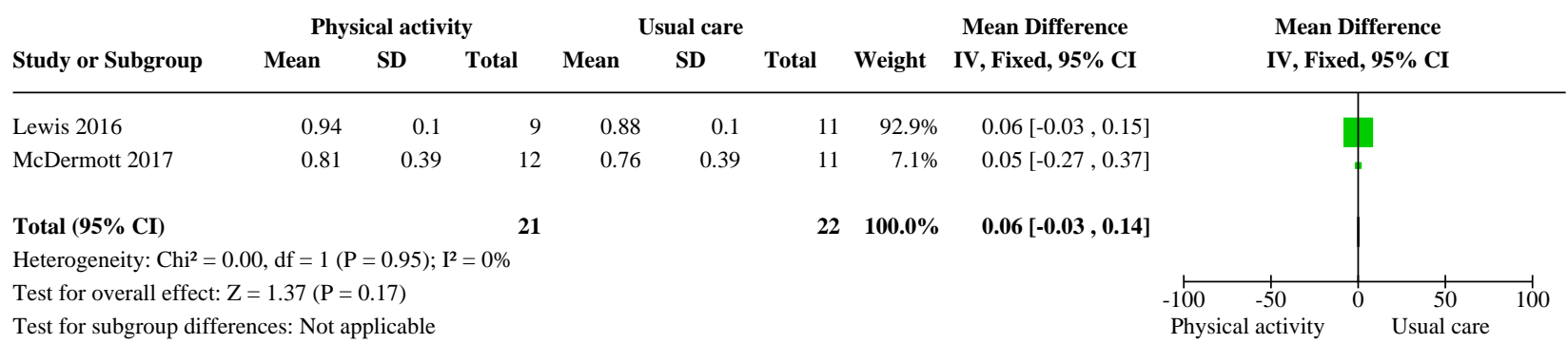

Comparison 10. Physical activity versus usual care for anthropometric measure of body fat (\%)

\begin{tabular}{llllll}
\hline Outcome or subgroup title & $\begin{array}{l}\text { No. of } \\
\text { studies }\end{array}$ & $\begin{array}{l}\text { No. of } \\
\text { partici- } \\
\text { pants }\end{array}$ & Statistical method & Effect size \\
\hline 10.1 Up to 12 weeks follow-up & 4 & 214 & Mean Difference (IV, Random, 95\% Cl) & $-1.93[-4.04,0.18]$ \\
\hline $\begin{array}{l}10.2 \text { Change from baseline up to } 12 \text { weeks } \\
\text { follow-up }\end{array}$ & 2 & 60 & Mean Difference (IV, Fixed, 95\% CI) & $-1.57[-3.11,-0.04]$ \\
\hline
\end{tabular}




\begin{tabular}{llllll}
\hline Outcome or subgroup title & $\begin{array}{l}\text { No. of } \\
\text { studies }\end{array}$ & $\begin{array}{l}\text { No. of } \\
\text { partici- } \\
\text { pants }\end{array}$ & Statistical method & Effect size \\
\hline $\begin{array}{l}10.3 \text { More than } 12 \text { weeks to } 6 \text { months fol- } \\
\text { low-up }\end{array}$ & 3 & 139 & $\begin{array}{l}\text { Std. Mean Difference (IV, Fixed, 95\% } \\
\text { Cl) }\end{array}$ & $-0.08[-0.42,0.27]$ \\
\hline $\begin{array}{l}\text { 10.4 Change from baseline more than 12 } \\
\text { weeks to 6 months follow-up }\end{array}$ & 2 & 51 & Mean Difference (IV, Fixed, 95\% CI) & $-1.26[-3.11,0.59]$ \\
\hline
\end{tabular}

Analysis 10.1. Comparison 10: Physical activity versus usual care for anthropometric measure of body fat (\%), Outcome 1: Up to 12 weeks follow-up

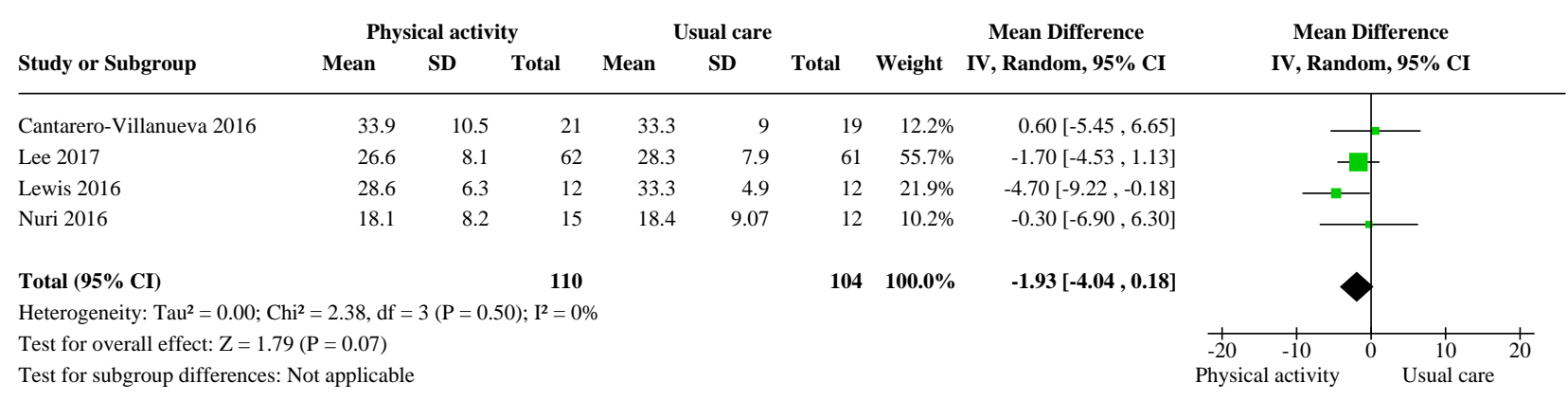

\section{Analysis 10.2. Comparison 10: Physical activity versus usual care for anthropometric measure of body fat (\%), Outcome 2: Change from baseline up to 12 weeks follow-up}

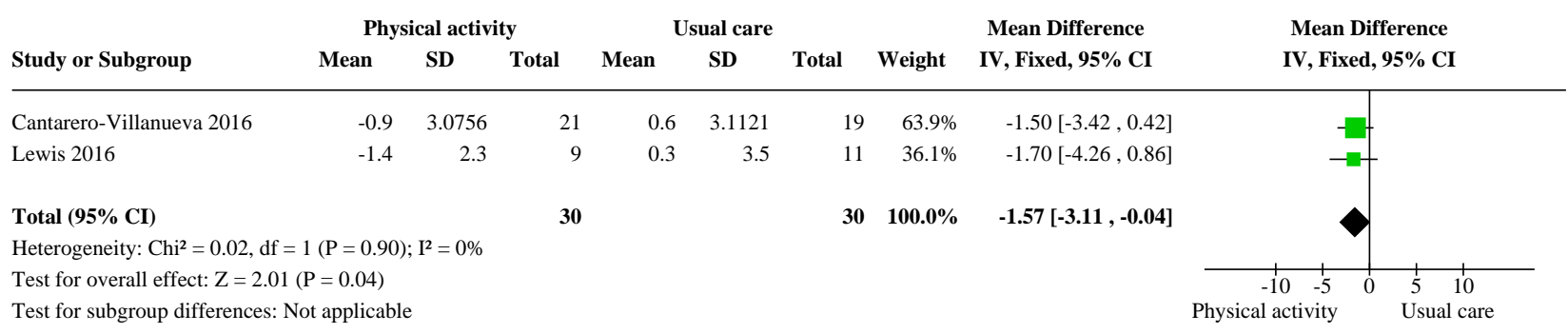

\section{Analysis 10.3. Comparison 10: Physical activity versus usual care for anthropometric measure of body fat (\%), Outcome 3: More than 12 weeks to 6 months follow-up}

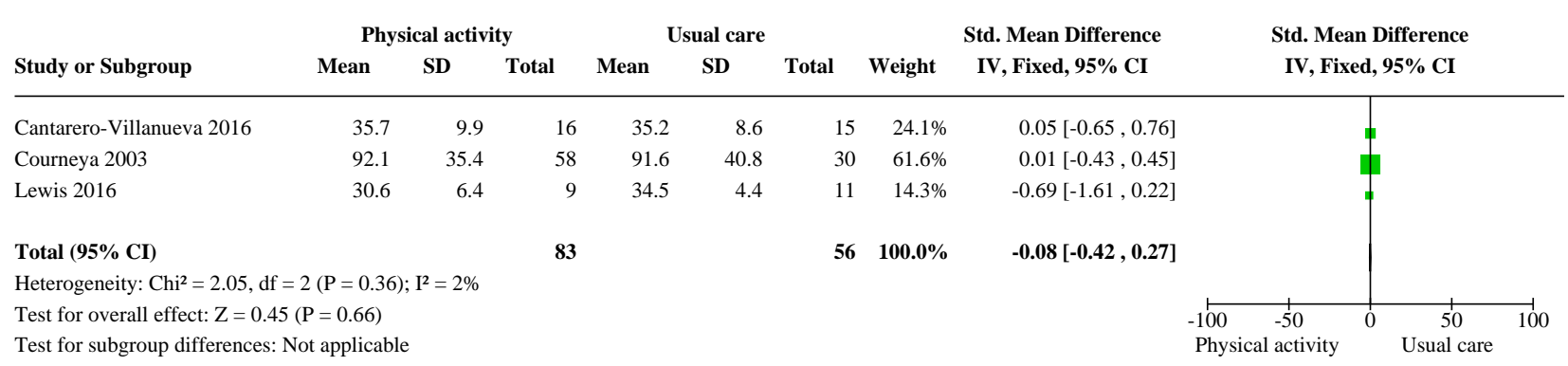


Analysis 10.4. Comparison 10: Physical activity versus usual care for anthropometric measure of body fat (\%), Outcome 4: Change from baseline more than 12 weeks to 6 months follow-up

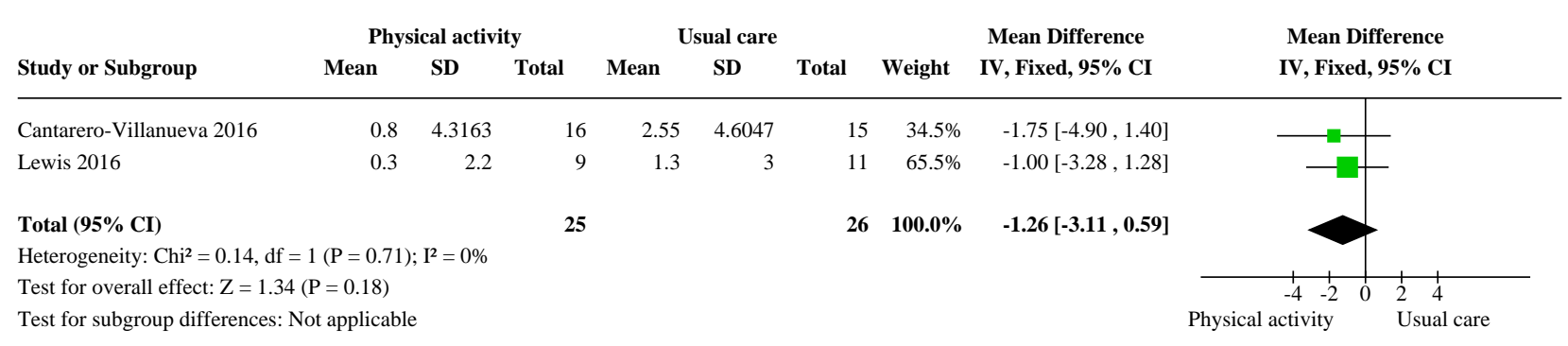

Comparison 11. Physical activity versus usual care for anthropometric measure of BMI

\begin{tabular}{llllll}
\hline Outcome or subgroup title & $\begin{array}{l}\text { No. of } \\
\text { studies }\end{array}$ & $\begin{array}{l}\text { No. of } \\
\text { partici- } \\
\text { pants }\end{array}$ & Statistical method & Effect size \\
\hline 11.1 Up to 12 weeks follow-up & 6 & 252 & Mean Difference (IV, Random, 95\% Cl) & $0.14[-0.73,1.02]$ \\
\hline $\begin{array}{l}11.2 \text { Change from baseline up to 12 weeks } \\
\text { follow-up }\end{array}$ & 3 & 82 & Mean Difference (IV, Random, 95\% Cl) & $-0.32[-0.81,0.17]$ \\
\hline $\begin{array}{l}11.3 \text { More than } 12 \text { weeks to 6 months fol- } \\
\text { low-up }\end{array}$ & 4 & 158 & Std. Mean Difference (IV, Random, 95\% & $0.00[-0.32,0.33]$ \\
\hline $\begin{array}{l}11.4 \text { Change from baseline more than 12 } \\
\text { weeks to 6 months follow-up }\end{array}$ & 3 & 89 & Cl) & \\
\hline
\end{tabular}

Analysis 11.1. Comparison 11: Physical activity versus usual care for anthropometric measure of BMI, Outcome 1: Up to 12 weeks follow-up

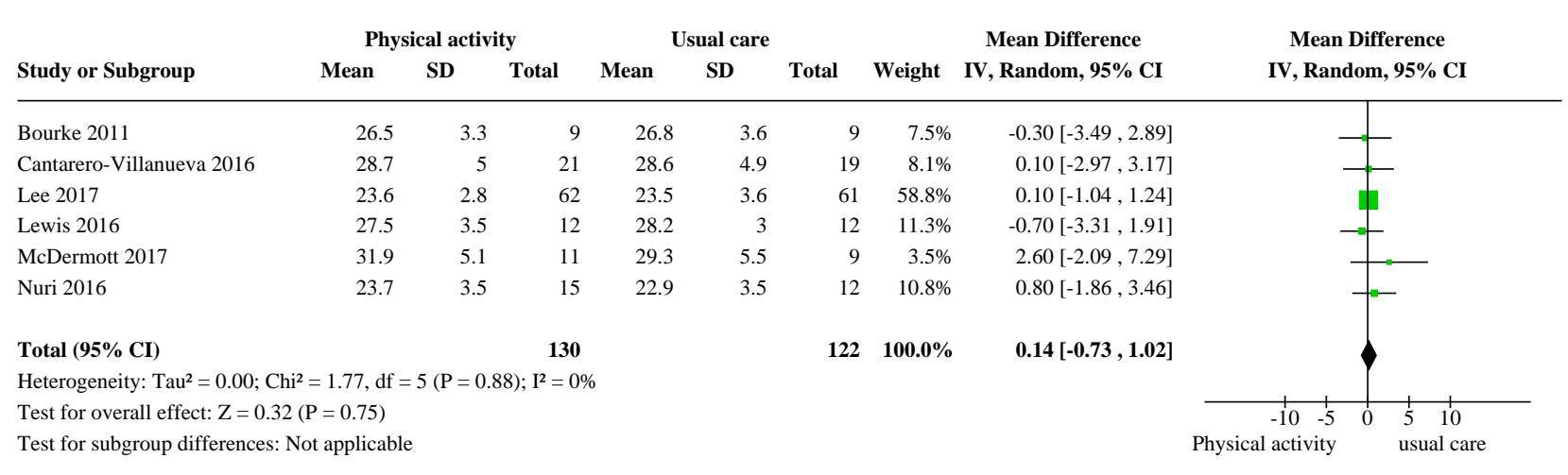


Analysis 11.2. Comparison 11: Physical activity versus usual care for anthropometric measure of BMI, Outcome 2: Change from baseline up to 12 weeks follow-up

\begin{tabular}{|c|c|c|c|c|c|c|c|c|c|}
\hline \multirow[b]{2}{*}{ Study or Subgroup } & \multicolumn{3}{|c|}{ Physical activity } & \multicolumn{3}{|c|}{ Usual care } & \multirow[b]{2}{*}{ Weight } & \multirow{2}{*}{$\begin{array}{c}\text { Mean Difference } \\
\text { IV, Random, } 95 \% \text { CI }\end{array}$} & \multirow{2}{*}{$\begin{array}{c}\text { Mean Difference } \\
\text { IV, Random, 95\% CI }\end{array}$} \\
\hline & Mean & SD & Total & Mean & SD & Total & & & \\
\hline Bourke 2011 & -0.4 & 0.6505 & 9 & -0.2 & 0.6505 & 9 & $37.2 \%$ & $-0.20[-0.80,0.40]$ & -1 \\
\hline Cantarero-Villanueva 2016 & 0.3 & 1.3181 & 21 & 0.3 & 0.6224 & 19 & $35.3 \%$ & $0.00[-0.63,0.63]$ & - \\
\hline Lewis 2016 & -0.4 & 0.8 & 12 & 0.5 & 1.1 & 12 & $27.5 \%$ & $-0.90[-1.67,-0.13]$ & $\longrightarrow$ \\
\hline Total $(95 \%$ CI $)$ & & & 42 & & & 40 & $100.0 \%$ & $-0.32[-0.81,0.17]$ & \\
\hline \multicolumn{10}{|c|}{ Heterogeneity: $\mathrm{Tau}^{2}=0.07 ; \mathrm{Chi}^{2}=3.31, \mathrm{df}=2(\mathrm{P}=0.19) ; \mathrm{I}^{2}=40 \%$} \\
\hline \multicolumn{9}{|c|}{ Test for overall effect: $\mathrm{Z}=1.28(\mathrm{P}=0.20)$} & $\begin{array}{ll}-2 & -1\end{array}$ \\
\hline \multicolumn{9}{|c|}{ Test for subgroup differences: Not applicable } & Physical activity \\
\hline
\end{tabular}

\section{Analysis 11.3. Comparison 11: Physical activity versus usual care for anthropometric} measure of BMI, Outcome 3: More than 12 weeks to 6 months follow-up

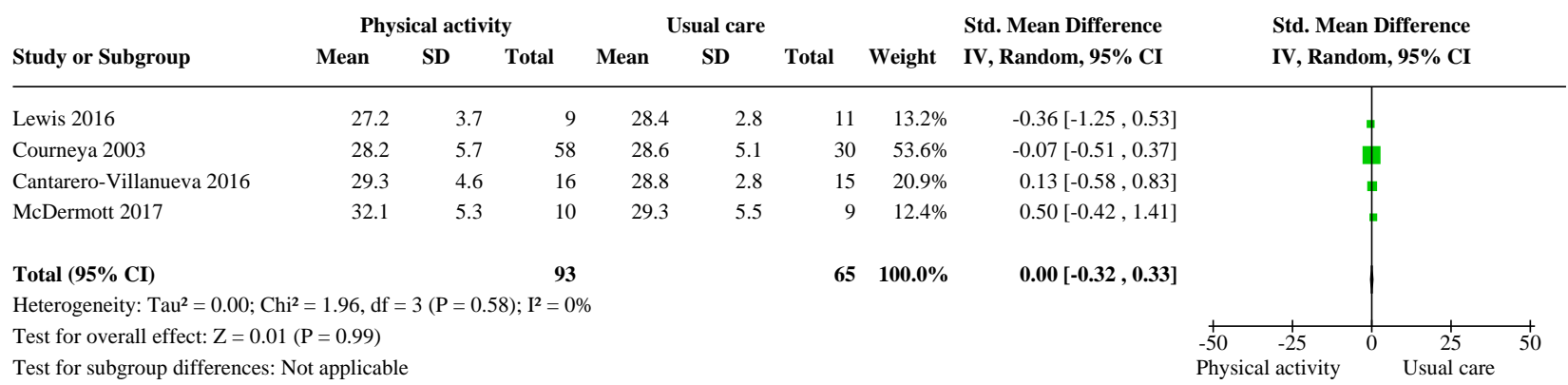

Analysis 11.4. Comparison 11: Physical activity versus usual care for anthropometric measure of BMI, Outcome 4: Change from baseline more than 12 weeks to 6 months follow-up

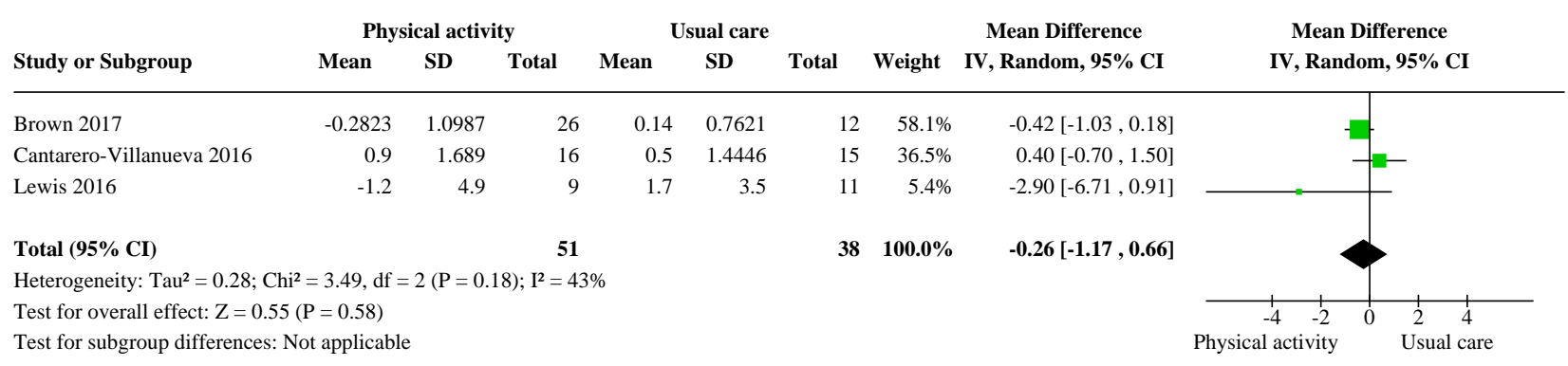

Comparison 12. Physical activity versus usual care for HRQOL

\begin{tabular}{llllll}
\hline Outcome or subgroup title & $\begin{array}{l}\text { No. of } \\
\text { studies }\end{array}$ & $\begin{array}{l}\text { No. of } \\
\text { partici- } \\
\text { pants }\end{array}$ & Statistical method & Effect size \\
\hline 12.1 Up to 12 weeks follow-up & 6 & 230 & Std. Mean Difference (IV, Random, 95\% Cl) & $0.36[0.10,0.62]$ \\
\hline $\begin{array}{l}12.2 \text { Change from baseline up to 12 } \\
\text { weeks follow-up }\end{array}$ & 3 & 113 & Std. Mean Difference (IV, Fixed, 95\% Cl) & $-0.10[-0.47,0.28]$ \\
\hline $\begin{array}{l}12.3 \text { More than } 12 \text { weeks to 6 months } \\
\text { follow-up }\end{array}$ & 7 & 278 & Std. Mean Difference (IV, Random, 95\% Cl) & $0.45[0.03,0.88]$ \\
\hline \hline
\end{tabular}

Physical activity interventions for disease-related physical and mental health during and following treatment in people with non- 


\begin{tabular}{llllll}
\hline Outcome or subgroup title & $\begin{array}{l}\text { No. of } \\
\text { studies }\end{array}$ & $\begin{array}{l}\text { No. of } \\
\text { partici- } \\
\text { pants }\end{array}$ & Statistical method & Effect size \\
\hline $\begin{array}{l}\text { 12.4 Change from baseline more than } 12 \\
\text { weeks to 6 months follow-up }\end{array}$ & 2 & 58 & Std. Mean Difference (IV, Fixed, 95\% CI) & $0.70[0.14,1.26]$ \\
\hline $\begin{array}{l}\text { 12.5 More than } 6 \text { months to } 12 \text { months } \\
\text { follow-up }\end{array}$ & 3 & 89 & Std. Mean Difference (IV, Fixed, 95\% Cl) & $0.05[-0.37,0.47]$ \\
\hline
\end{tabular}

Analysis 12.1. Comparison 12: Physical activity versus usual care for HRQoL, Outcome 1: Up to 12 weeks follow-up

\begin{tabular}{|c|c|c|c|c|c|c|c|c|c|}
\hline \multirow[b]{2}{*}{ Study or Subgroup } & \multicolumn{3}{|c|}{ Physical activity } & \multicolumn{3}{|c|}{ Usual care } & \multirow[b]{2}{*}{ Weight } & \multirow{2}{*}{$\begin{array}{l}\text { Std. Mean Difference } \\
\text { IV, Random, 95\% CI }\end{array}$} & \multirow{2}{*}{$\begin{array}{l}\text { Std. Mean Difference } \\
\text { IV, Random, 95\% CI }\end{array}$} \\
\hline & Mean & SD & Total & Mean & SD & Total & & & \\
\hline Bourke 2011 & 120 & 11 & 9 & 106 & 13 & 9 & $6.8 \%$ & $1.11[0.10,2.12]$ & \\
\hline Cramer 2016 & 110.66 & 13.26 & 27 & 106.44 & 18.46 & 27 & $24.1 \%$ & $0.26[-0.28,0.79]$ & \\
\hline Kim 2018 & 104.3 & 17.5 & 37 & 99.1 & 19.1 & 34 & $31.6 \%$ & $0.28[-0.19,0.75]$ & \\
\hline Lewis 2016 & 98.8 & 7.3 & 12 & 91.9 & 12.3 & 12 & $10.1 \%$ & $0.66[-0.17,1.48]$ & \\
\hline McDermott 2017 & 117 & 12 & 11 & 104 & 23 & 9 & $8.3 \%$ & $0.70[-0.21,1.62]$ & \\
\hline Pinto 2013 & 111.3 & 11.2037 & 19 & 110.8 & 11.1305 & 24 & $19.1 \%$ & $0.04[-0.56,0.65]$ & \\
\hline Total $(95 \%$ CI $)$ & & & 115 & & & 115 & $100.0 \%$ & $0.36[0.10,0.62]$ & \\
\hline \multicolumn{10}{|c|}{ Heterogeneity: $\mathrm{Tau}^{2}=0.00 ; \mathrm{Chi}^{2}=4.44, \mathrm{df}=5(\mathrm{P}=0.49) ; \mathrm{I}^{2}=0 \%$} \\
\hline \multicolumn{9}{|c|}{ Test for overall effect: $\mathrm{Z}=2.68(\mathrm{P}=0.007)$} & $\begin{array}{ccc}1 & 1 & 1 \\
-0.2 & -0.1 & 0\end{array}$ \\
\hline \multicolumn{9}{|c|}{ Test for subgroup differences: Not applicable } & Usual care \\
\hline
\end{tabular}

\section{Analysis 12.2. Comparison 12: Physical activity versus usual care for HRQOL, Outcome 2: Change from baseline up to 12 weeks follow-up}

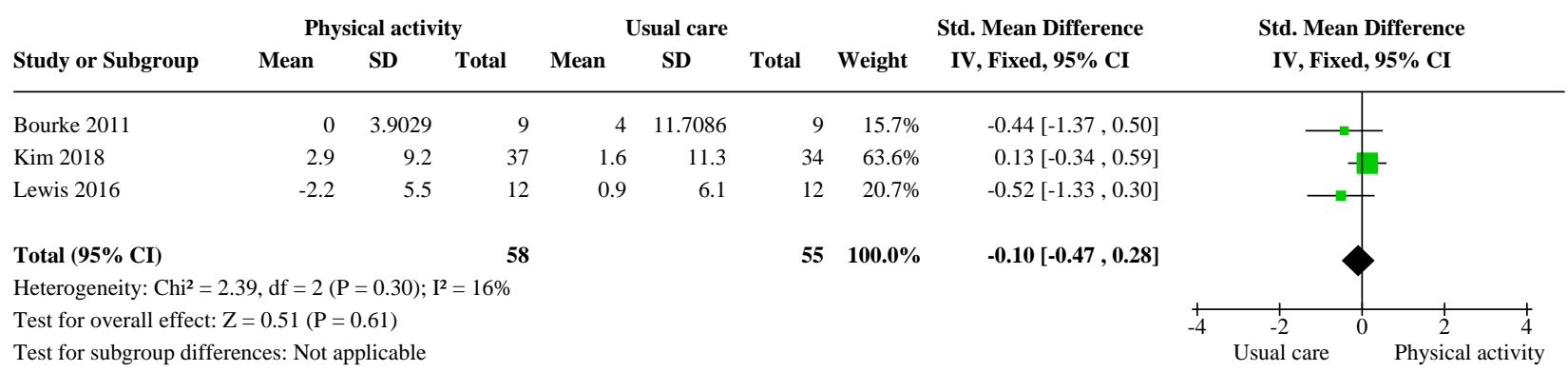




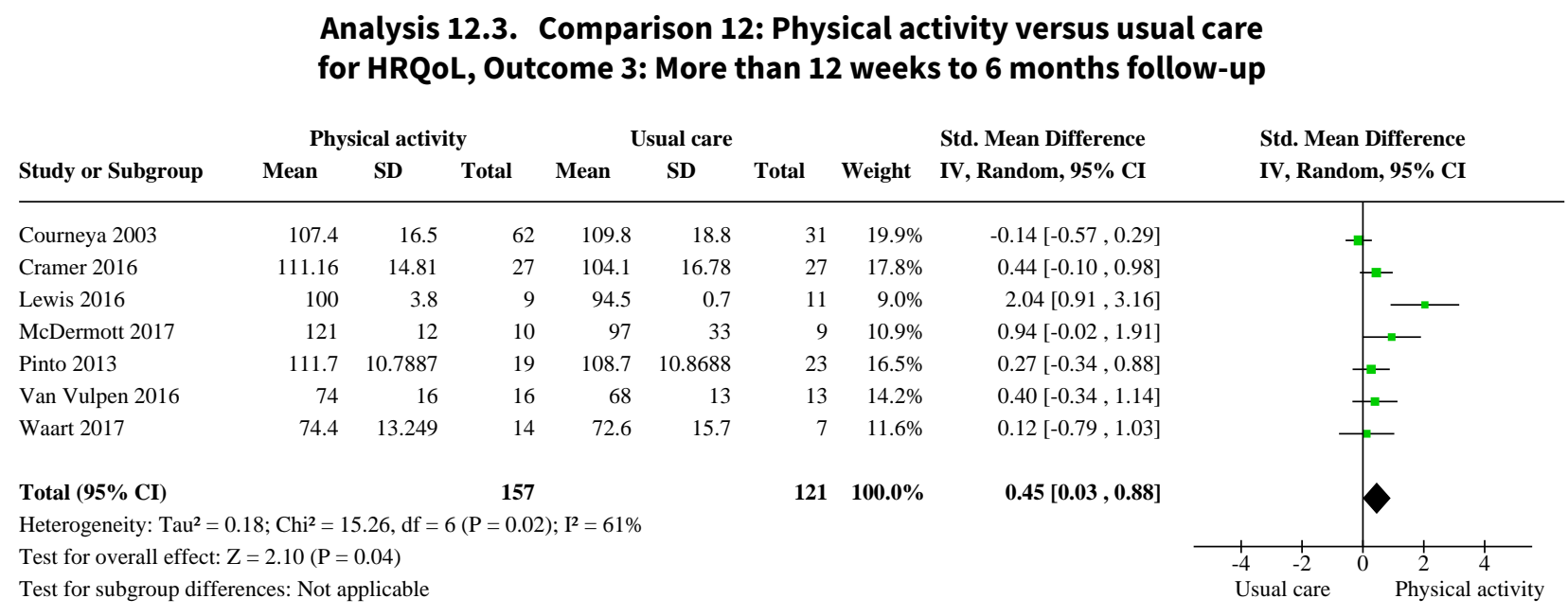

Analysis 12.4. Comparison 12: Physical activity versus usual care for HRQoL, Outcome 4: Change from baseline more than 12 weeks to 6 months follow-up

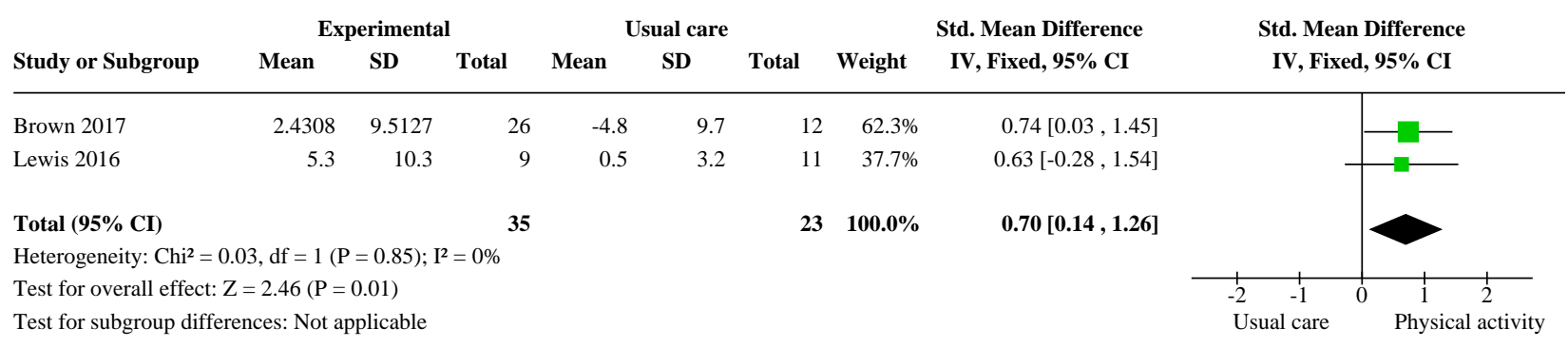

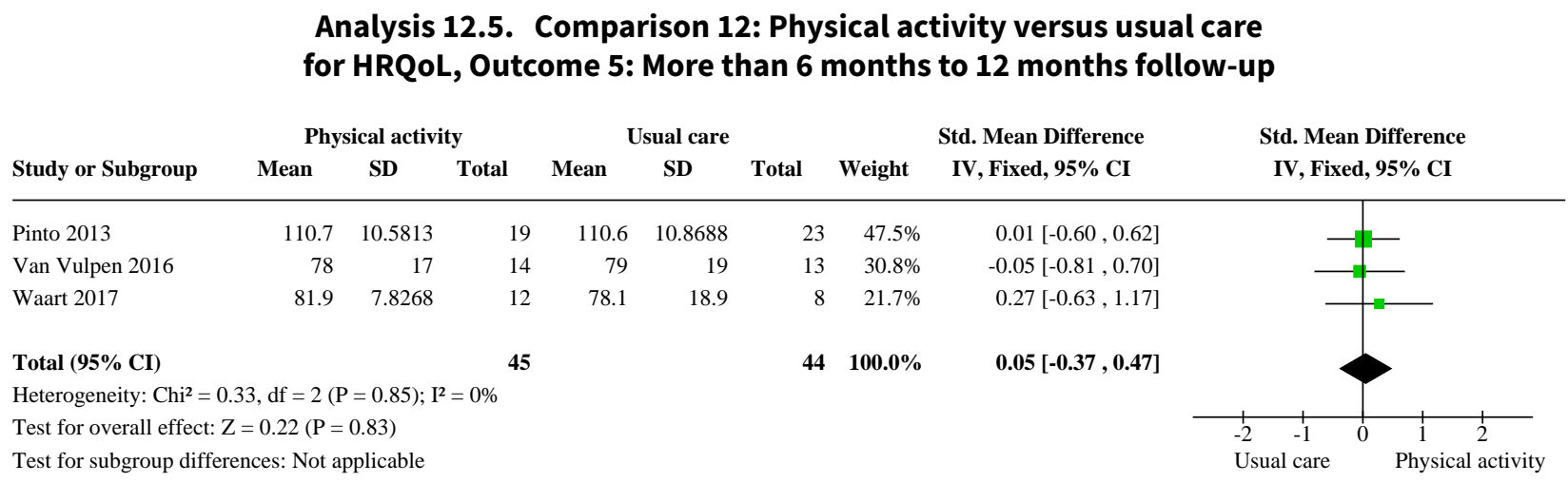

Comparison 13. Physical activity versus usual care for levels of physical activity

\begin{tabular}{lllll}
\hline Outcome or subgroup title & $\begin{array}{l}\text { No. of } \\
\text { studies }\end{array}$ & $\begin{array}{l}\text { No. of } \\
\text { partici- } \\
\text { pants }\end{array}$ & Statistical method & Effect size \\
\hline $\begin{array}{l}13.1 \text { Objective measures up to } 12 \text { weeks follow-up (ac- } \\
\text { celerometry moderate to vigorous physical activity mins/ } \\
\text { per day) }\end{array}$ & 4 & 94 & $\begin{array}{l}\text { Mean Difference (IV, Ran- } \\
\text { dom, 95\% Cl) }\end{array}$ & $\begin{array}{l}-8.34 \\
{[-21.05,} \\
4.37]\end{array}$ \\
\hline
\end{tabular}




\begin{tabular}{|c|c|c|c|c|}
\hline Outcome or subgroup title & $\begin{array}{l}\text { No. of } \\
\text { studies }\end{array}$ & $\begin{array}{l}\text { No. of } \\
\text { partici- } \\
\text { pants }\end{array}$ & Statistical method & Effect size \\
\hline $\begin{array}{l}13.2 \text { Change from baseline in objective measures up to } \\
12 \text { weeks follow-up (accelerometry moderate to vigorous } \\
\text { physical activity) }\end{array}$ & 2 & 37 & $\begin{array}{l}\text { Std. Mean Difference (IV, } \\
\text { Fixed, } 95 \% \mathrm{CI} \text { ) }\end{array}$ & $\begin{array}{l}-0.13[-0.77, \\
0.52]\end{array}$ \\
\hline 13.3 Subjective measures up to 12 weeks follow-up & 4 & 156 & $\begin{array}{l}\text { Std. Mean Difference (IV, } \\
\text { Random, 95\% CI) }\end{array}$ & $\begin{array}{l}0.70[0.38 \\
1.03]\end{array}$ \\
\hline $\begin{array}{l}13.4 \text { Objective measures more than } 12 \text { weeks to } 6 \text { months } \\
\text { follow-up (accelerometry moderate to vigorous physical } \\
\text { activity mins/week) }\end{array}$ & 2 & 36 & $\begin{array}{l}\text { Mean Difference (IV, Fixed, } \\
95 \% \mathrm{CI})\end{array}$ & $\begin{array}{l}13.50 \\
{[-56.73} \\
83.74]\end{array}$ \\
\hline $\begin{array}{l}13.5 \text { Subjective measures more than } 12 \text { weeks to } 6 \text { months } \\
\text { follow-up }\end{array}$ & 4 & 176 & $\begin{array}{l}\text { Std. Mean Difference (IV, } \\
\text { Random, } 95 \% \mathrm{CI} \text { ) }\end{array}$ & $\begin{array}{l}0.39[-0.05 \\
0.82]\end{array}$ \\
\hline $\begin{array}{l}\text { 13.6 Subjective measures more than } 6 \text { months to } 12 \\
\text { months follow-up }\end{array}$ & 3 & 274 & $\begin{array}{l}\text { Std. Mean Difference (IV, } \\
\text { Fixed, } 95 \% \mathrm{CI} \text { ) }\end{array}$ & $\begin{array}{l}0.35[0.11 \\
0.59]\end{array}$ \\
\hline
\end{tabular}

Analysis 13.1. Comparison 13: Physical activity versus usual care for levels of physical activity, Outcome 1: Objective measures up to 12 weeks follow-up (accelerometry moderate to vigorous physical activity mins/per day)

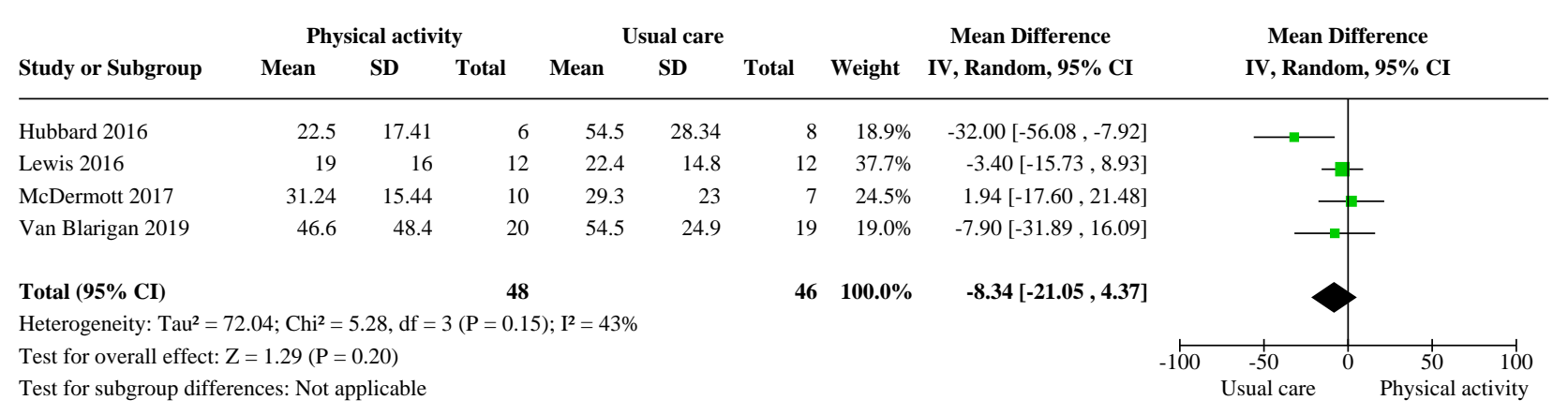

\begin{abstract}
Analysis 13.2. Comparison 13: Physical activity versus usual care for levels of physical activity, Outcome 2: Change from baseline in objective measures up to 12 weeks follow-up (accelerometry moderate to vigorous physical activity)
\end{abstract}

\begin{tabular}{|c|c|c|c|c|c|c|c|c|c|}
\hline \multirow[b]{2}{*}{ Study or Subgroup } & \multicolumn{3}{|c|}{ Physical activity } & \multicolumn{3}{|c|}{ Usual care } & \multirow[b]{2}{*}{ Weight } & \multirow{2}{*}{$\begin{array}{l}\text { Std. Mean Difference } \\
\text { IV, Fixed, 95\% CI }\end{array}$} & \multirow{2}{*}{$\begin{array}{l}\text { Std. Mean Difference } \\
\text { IV, Fixed, 95\% CI }\end{array}$} \\
\hline & Mean & SD & Total & Mean & SD & Total & & & \\
\hline Hubbard 2016 & 1.3 & 15.04 & 6 & 10.5 & 28.37 & 7 & $34.5 \%$ & $-0.37[-1.47,0.74]$ & \\
\hline Lewis 2016 & 27 & 91 & 12 & 27 & 92 & 12 & $65.5 \%$ & $0.00[-0.80,0.80]$ & \\
\hline Total $(95 \%$ CI $)$ & & & 18 & & & 19 & $100.0 \%$ & $-0.13[-0.77,0.52]$ & \\
\hline \multicolumn{10}{|c|}{ Heterogeneity: $\mathrm{Chi}^{2}=0.28, \mathrm{df}=1(\mathrm{P}=0.60) ; \mathrm{I}^{2}=0 \%$} \\
\hline \multicolumn{9}{|c|}{ Test for overall effect: $\mathrm{Z}=0.38(\mathrm{P}=0.70)$} & $-2 \quad-1$ \\
\hline \multicolumn{9}{|c|}{ Test for subgroup differences: Not applicable } & Usual care \\
\hline
\end{tabular}


Analysis 13.3. Comparison 13: Physical activity versus usual care for levels of physical activity, Outcome 3: Subjective measures up to 12 weeks follow-up

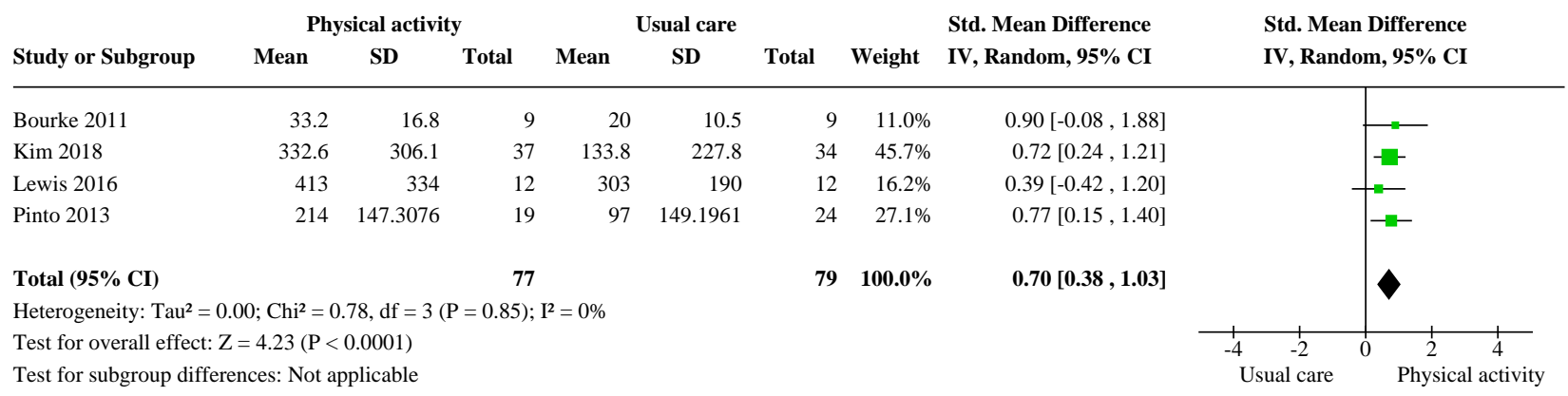

Analysis 13.4. Comparison 13: Physical activity versus usual care for levels of physical activity, Outcome 4: Objective measures more than 12 weeks to 6 months follow-up (accelerometry moderate to vigorous physical activity mins/week)

\begin{tabular}{|c|c|c|c|c|c|c|c|c|c|}
\hline \multirow[b]{2}{*}{ Study or Subgroup } & \multicolumn{3}{|c|}{ Physical activity } & \multicolumn{3}{|c|}{ Usual care } & \multirow[b]{2}{*}{ Weight } & \multirow{2}{*}{$\begin{array}{l}\text { Mean Difference } \\
\text { IV, Fixed, 95\% CI }\end{array}$} & \multirow{2}{*}{$\begin{array}{l}\text { Mean Difference } \\
\text { IV, Fixed, 95\% CI }\end{array}$} \\
\hline & Mean & SD & Total & Mean & SD & Total & & & \\
\hline Lewis 2016 & 121 & 99 & 9 & 152 & 145 & 11 & $42.8 \%$ & $-31.00[-138.36,76.36]$ & -1 \\
\hline McDermott 2017 & 177.6 & 89.1 & 10 & 130.8 & 93.3 & 6 & $57.2 \%$ & $46.80[-46.06,139.66]$ & \\
\hline Total $(95 \%$ CI $)$ & & & 19 & & & 17 & $100.0 \%$ & $13.50[-56.73,83.74]$ & \\
\hline \multicolumn{10}{|c|}{ Heterogeneity: $\mathrm{Chi}^{2}=1.15, \mathrm{df}=1(\mathrm{P}=0.28) ; \mathrm{I}^{2}=13 \%$} \\
\hline \multicolumn{9}{|c|}{ Test for overall effect: $\mathrm{Z}=0.38(\mathrm{P}=0.71)$} & $-200-100$ \\
\hline \multicolumn{9}{|c|}{ Test for subgroup differences: Not applicable } & Usual care \\
\hline
\end{tabular}

Analysis 13.5. Comparison 13: Physical activity versus usual care for levels of physical activity, Outcome 5: Subjective measures more than 12 weeks to 6 months follow-up

\begin{tabular}{|c|c|c|c|c|c|c|c|c|c|}
\hline \multirow[b]{2}{*}{ Study or Subgroup } & \multicolumn{3}{|c|}{ Physical activity } & \multicolumn{3}{|c|}{ Usual care } & \multirow[b]{2}{*}{ Weight } & \multirow{2}{*}{$\begin{array}{l}\text { Std. Mean Difference } \\
\text { IV, Random, 95\% CI }\end{array}$} & \multirow{2}{*}{$\begin{array}{l}\text { Std. Mean Difference } \\
\text { IV, Random, 95\% CI }\end{array}$} \\
\hline & Mean & SD & Total & Mean & SD & Total & & & \\
\hline Courneya 2003 & 193.52 & 146.36 & 62 & 180.12 & 185.82 & 31 & $39.8 \%$ & $0.08[-0.35,0.51]$ & - \\
\hline Lewis 2016 & 663 & 261 & 9 & 332 & 253 & 11 & $15.1 \%$ & $1.24[0.26,2.21]$ & \\
\hline Pinto 2013 & 160 & 145.2329 & 19 & 89 & 145.6875 & 23 & $28.2 \%$ & $0.48[-0.14,1.10]$ & \\
\hline Waart 2017 & 99.6 & 64.791 & 14 & 88.7 & 35.5 & 7 & $16.9 \%$ & $0.18[-0.73,1.09]$ & \\
\hline Total $(95 \%$ CI $)$ & & & 104 & & & 72 & $100.0 \%$ & $0.39[-0.05,0.82]$ & \\
\hline \multicolumn{10}{|c|}{ Heterogeneity: $\mathrm{Tau}^{2}=0.07 ; \mathrm{Chi}^{2}=4.86, \mathrm{df}=3(\mathrm{P}=0.18) ; \mathrm{I}^{2}=38 \%$} \\
\hline \multirow{2}{*}{\multicolumn{9}{|c|}{$\begin{array}{l}\text { Test for overall effect: } \mathrm{Z}=1.74(\mathrm{P}=0.08) \\
\text { Test for subgroup differences: Not applicable }\end{array}$}} & 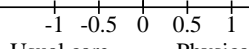 \\
\hline & & & & & & & & & Usual care \\
\hline
\end{tabular}


Analysis 13.6. Comparison 13: Physical activity versus usual care for levels of physical activity, Outcome 6: Subjective measures more than 6 months to 12 months follow-up

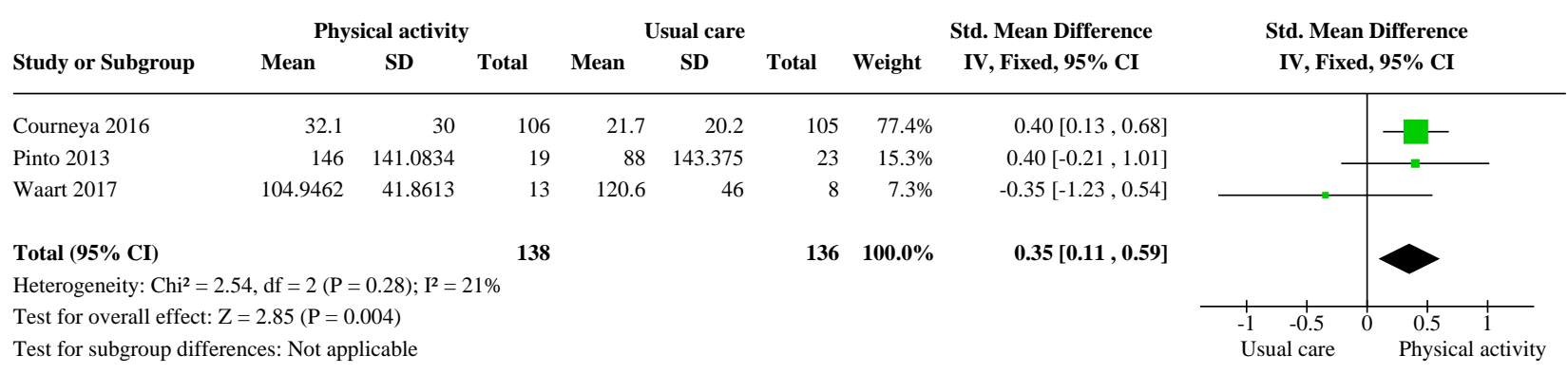

ADDITIONAL TABLES

Table 1. Summary of sensitivity analysis

\begin{tabular}{|c|c|c|c|c|c|}
\hline Outcome & Time point & $\begin{array}{l}\text { No. of } \\
\text { studies }\end{array}$ & $\begin{array}{l}\text { No. of } \\
\text { partici- } \\
\text { pants }\end{array}$ & Statistical method & Effect size \\
\hline \multicolumn{6}{|l|}{1 Physical function $d$} \\
\hline $\begin{array}{l}\text { 1.1 Subjective measure } \\
\text { of physical function }\end{array}$ & Short-term follow-up & 2 & 114 & $\begin{array}{l}\text { SMD (IV, random, 95\% } \\
\mathrm{CI} \text { ) }\end{array}$ & $0.08(-0.31 \text { to } 0.47)^{a}$ \\
\hline \multicolumn{6}{|l|}{$\begin{array}{l}2 \text { Disease-related men- } \\
\text { tal health }\end{array}$} \\
\hline 2.1 Anxiety & Short-term follow-up & 3 & 177 & $\begin{array}{l}\text { SMD (IV, random, 95\% } \\
\mathrm{CI})\end{array}$ & $-0.29(-0.60 \text { to } 0.01)^{a, b}$ \\
\hline 2.2. Depression & Short-term follow-up & 3 & 177 & $\begin{array}{l}\text { SMD (IV, random, 95\% } \\
\text { CI) }\end{array}$ & $-0.18(-0.48 \text { to } 0.13)^{a, b}$ \\
\hline \multicolumn{6}{|l|}{3 Physical fitness } \\
\hline 3.1 Aerobic fitness & $\begin{array}{l}\text { Immediate-term fol- } \\
\text { low-up } \\
\text { Short-term follow-up }\end{array}$ & $\begin{array}{l}4 \\
5\end{array}$ & $\begin{array}{l}207 \\
187\end{array}$ & $\begin{array}{l}\text { SMD (IV, random, 95\% } \\
\mathrm{CI})\end{array}$ & $\begin{array}{l}0.38(0.06 \text { to } 0.70)^{a, b} \\
0.45(0.15 \text { to } 0.75)^{a, b}\end{array}$ \\
\hline $\begin{array}{l}4 \text { Cancer-related fa- } \\
\text { tigue } d\end{array}$ & $\begin{array}{l}\text { Immediate-term fol- } \\
\text { low-up } \\
\text { Short-term follow-up }\end{array}$ & $\begin{array}{l}4 \\
5\end{array}$ & $\begin{array}{l}169 \\
224\end{array}$ & $\begin{array}{l}\text { MD (IV, random, 95\% CI) } \\
\text { SMD (IV, random, 95\% } \\
\mathrm{CI})\end{array}$ & $\begin{array}{l}2.22(-0.34 \text { to } 4.79)^{a, b} \\
0.32(-0.04 \text { to } 0.67)^{a, b}\end{array}$ \\
\hline
\end{tabular}

\section{Anthropometric mea-}

sures $d$

\begin{tabular}{llllll}
\hline 5.1 Weight & Immediate-term fol- & 4 & 207 & MD (IV, random, 95\% Cl) & $0.27(-2.87$ to 3.42)a,c \\
& low-up & 2 & 64 & & $-1.76[-4.06$ to 0.54]c \\
& $\begin{array}{l}\text { Change from base- } \\
\text { line to } 12 \text { weeks fol- } \\
\text { low-up }\end{array}$ & &
\end{tabular}


Table 1. Summary of sensitivity analysis (Continued)

\begin{tabular}{|c|c|c|c|c|c|}
\hline 5.2 Waist to hip ratio & $\begin{array}{l}\text { Immediate-term fol- } \\
\text { low-up }\end{array}$ & 2 & 44 & MD (IV, random, 95\% Cl) & $0.04[-0.01 \text { to } 0.10]^{a, c}$ \\
\hline $5.3 \mathrm{BMI}$ & $\begin{array}{l}\text { Immediate-term fol- } \\
\text { low-up } \\
\text { Change from base- } \\
\text { line to } 12 \text { weeks fol- } \\
\text { low-up }\end{array}$ & 2 & $\begin{array}{l}207 \\
64\end{array}$ & MD (IV, random, 95\% CI) & $\begin{array}{l}0.10[-0.87 \text { to } 1.06]^{a, c} \\
-0.42[-1.30 \text { to } 0.46]\end{array}$ \\
\hline 5.4 Body fat $\%$ & $\begin{array}{l}\text { Immediate-term fol- } \\
\text { low-up }\end{array}$ & 3 & 187 & MD (IV, random, 95\% CI) & $-2.13[-4.46 \text { to } 0.21]^{a, d}$ \\
\hline 6 HRQOL & $\begin{array}{l}\text { Immediate-term fol- } \\
\text { low-up }\end{array}$ & 4 & 169 & $\begin{array}{l}\text { SMD (IV, random, 95\% } \\
\text { CI) }\end{array}$ & $0.37[0.07 \text { to } 0.68]^{a, b, c}$ \\
\hline \multicolumn{6}{|l|}{$\begin{array}{l}7 \text { Levels of physical ac- } \\
\text { tivity }\end{array}$} \\
\hline 7.1 Objective measures & $\begin{array}{l}\text { Immediate-term fol- } \\
\text { low-up }\end{array}$ & 3 & 80 & MD (IV, random, 95\% CI) & $-2.84[-12.40 \text { to } 6.73]^{c}$ \\
\hline 7.2 Subjective measures & $\begin{array}{l}\text { Immediate-term fol- } \\
\text { low-up }\end{array}$ & 3 & 138 & $\begin{array}{l}\text { SMD (IV, random, 95\% } \\
\mathrm{Cl} \text { ) }\end{array}$ & $0.68[0.33 \text { to } 1.02]^{c}$ \\
\hline
\end{tabular}

BMI: body mass index; CI: confidence interval; HRQoL: health-related quality of life; MD: mean difference: SD: standard deviation; SMD: standardised mean difference (used when studies assess the same outcome but measure it in a variety of ways).

$a$ Removal of studies that did not conduct an ITT analysis

$b$ Exclusion of studies at high risk of bias

c Exclusion of studies with an additional intervention component

$d$ Results from choice of model (fixed or random) were consistent

\section{AP P E N D I CES}

\section{Appendix 1. CENTRAL search strategy}

Cochrane Central Register of Controlled Trials (CENTRAL): year, issue number in the Cochrane Library (searched day, month, year)

\#1 MeSH descriptor: [Colorectal Neoplasms] explode all trees

\#2 ((colorect* or colon $^{\star}$ or rect* or anal $^{\star}$ or anus ${ }^{\star}$ or intestin* ${ }^{\star}$ or bowel $\left.{ }^{\star}\right)$ near $/ 5$ (carcinom* or neoplas* or adenocarcinom ${ }^{\star}$ or cancer $^{\star}$ or tumor $^{\star}$ or tumour ${ }^{\star}$ or sarcom ${ }^{\star}$ or malignan $\left.\left.{ }^{\star}\right)\right):$ ti,ab,kw (Word variations have been searched)

\#3 \#1 or \#2

\#4 MeSH descriptor: [Exercise] explode all trees

\#5 MeSH descriptor: [Exercise Therapy] explode all trees

\#6 MeSH descriptor: [Sports] explode all trees

\#7 "physical fitness" (Word variations have been searched)

\#8 (physical ${ }^{\star}$ near/5 (fit* or train $^{\star}$ or activ* or endur $^{\star}$ or exer $\left.^{\star}\right)$ ):ti,ab,kw (Word variations have been searched)

\#9 (exercis ${ }^{\star}$ near/5 (train* or physical* or activ*)):ti,ab,kw (Word variations have been searched)

\#10 sport*:ti,ab,kw (Word variations have been searched) 
\#11 walk*:ti,ab,kw (Word variations have been searched)

\#12 swim*:ti,ab,kw (Word variations have been searched)

\#13 pilates*:ti,ab,kw (Word variations have been searched)

\#14 tai ji or tai chi or tai-ji or tai-chi:ti,ab,kw (Word variations have been searched)

\#15 resistance near/3 train*:ti,ab,kw (Word variations have been searched)

$\# 16 \# 4$ and \#5 or \#6 or \#7 or \#8 or \#9 or \#10 or \#11 or \#12 or \#13 or \#14 or \#15

$\# 17$ \#3 and \#16

Appendix 2. MEDLINE search strategy

Ovid MEDLINE(R) Epub Ahead of Print, In-Process \& Other Non-Indexed Citations, Ovid MEDLINE(R) Daily and Ovid MEDLINE(R) 1946 to present (day, month, year)

1. exp colorectal neoplasms/

2. ((colorect ${ }^{\star}$ or colon ${ }^{\star}$ or rect ${ }^{\star}$ or anal ${ }^{\star}$ or anus ${ }^{\star}$ or intestin ${ }^{\star}$ or bowel $\left.{ }^{\star}\right)$ adj5 (carcinom ${ }^{\star}$ or neoplas ${ }^{\star}$ or adenocarcinom ${ }^{\star}$ or cancer $^{\star}$ or tumor $^{\star}$ or tumour ${ }^{\star}$ or sarcom ${ }^{\star}$ or malignan $\left.{ }^{\star}\right)$ ).mp.

\section{1 or 2}

4. exp exercise/

5. exp exercise therapy/

6. exp sports/

7. Physical Fitness/

8. (physical ${ }^{\star}$ adj5 (fit ${ }^{\star}$ or train ${ }^{\star}$ or activ* or endur $^{\star}$ or exer $\left.{ }^{\star}\right)$ ).ti,ab.

9. (exercis* $\operatorname{adj}$ (train* or physical $^{\star}$ or $\left.\left.\operatorname{activ}^{\star}\right)\right) \cdot \mathrm{ti}, \mathrm{ab}$.

10. sport*.ti,ab.

11. walk*.ti,ab.

12. swim ${ }^{\star} . \mathrm{ti}, \mathrm{ab}$.

13. pilates.ti,ab.

14. (tai ji or tai chi or tai-ji or tai-chi).ti,ab.

15. (resistance adj3 train $\left.{ }^{\star}\right) . t i, a b$.

16.4 or 5 or 6 or 7 or 8 or 9 or 10 or 11 or 12 or 13 or 14 or 15

17.3 and 16

18. randomized controlled trial.pt.

19. controlled clinical trial.pt. 
(Continued)

20. randomized.ab.

21. placebo.ab.

22. clinical trials as topic.sh.

23. randomly.ab.

24. trial.ti.

\subsection{8 or 19 or 20 or 21 or 22 or 23 or 24}

26. exp animals/ not humans.sh.

\subsection{5 not 26}

28. 17 and 27

\section{Appendix 3. Embase search strategy}

\section{Ovid Embase: 1974 to year week}

1. exp large intestine tumor/

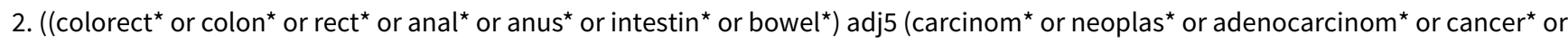
tumor $^{\star}$ or tumour $^{\star}$ or sarcom $^{\star}$ or malignan $\left.{ }^{\star}\right)$ ).mp.

3. 1 or 2

\section{4. exp exercise/}

5. exp sport/

6. physical fitness/

7. exercise therapy/

8. (physical ${ }^{\star}$ adj5 (fit ${ }^{\star}$ or train* ${ }^{\star}$ activ ${ }^{\star}$ or endur ${ }^{\star}$ or exer $\left.{ }^{\star}\right)$ ).ti,ab.

9. $\left(\right.$ exercis $^{\star}$ adj5 (train* or physical ${ }^{\star}$ or activ $\left.\left.^{\star}\right)\right) \cdot$ ti,ab.

\section{0. sport*.ti,ab.}

11. walk*.ti,ab.

12. swim ${ }^{\star} . t i, a b$.

13. pilates.ti,ab.

14. (tai ji or tai chi or tai-ji or tai-chi).ti,ab.

15. (resistance adj3 train*).ti,ab. 
(Continued)

16.4 or 5 or 6 or 7 or 8 or 9 or 10 or 11 or 12 or 13 or 14 or 15

\section{3 and 16}

18. CROSSOVER PROCEDURE.sh.

19. DOUBLE-BLIND PROCEDURE.sh.

20. SINGLE-BLIND PROCEDURE.sh.

21. (crossover ${ }^{\star}$ or cross over $\left.^{\star}\right)$.ti,ab.

22. placebo*.ti,ab.

23. (doubl ${ }^{\star}$ adj blind $\left.{ }^{\star}\right)$.ti,ab.

24. allocat*.ti,ab.

25. trial.ti.

26. RANDOMIZED CONTROLLED TRIAL.sh.

27. random*.ti,ab.

28.18 or 19 or 20 or 21 or 22 or 23 or 24 or 25 or 26 or 27

29. (exp animal/ or exp invertebrate/ or animal.hw. or nonhuman/) not (exp human/ or human cell/ or (human or humans or man or men or wom?n).ti.)

30.28 not 29

31.17 and 30

\section{Appendix 4. Criteria for judging risk of bias in the 'Risk of bias' assessment tool}

\section{Random sequence generation}

\section{Selection bias (biased allocation to interventions) due to inadequate generation of a randomised sequence}

Criteria for a judgement of 'low risk' of bias
The investigators describe a random component in the sequence generation process such as:

1. referring to a random number table;

2. using a computer random number generator;

3. coin tossing;

4. shuffling cards or envelopes;

5. throwing dice;

6. drawing of lots;

7. minimisation*.

*Minimisation may be implemented without a random element, and this is considered to be equivalent to being random. 
(Continued)

Criteria for the judgement of 'high risk' of bias
The investigators describe a non-random component in the sequence generation process. Usually, the description would involve some systematic, non-random approach, for example:

1. sequence generated by odd or even date of birth;

2. sequence generated by some rule based on date (or day) of admission;

3. sequence generated by some rule based on hospital or clinic record number.

Other non-random approaches happen much less frequently than the systematic approaches mentioned above and tend to be obvious. They usually involve judgement or some method of non-random categorisation of participants, for example:

1. allocation by judgement of the clinician;

2. allocation by preference of the participant;

3. allocation based on the results of a laboratory test or a series of tests;

4. allocation by availability of the intervention.
Criteria for the judgement of 'unclear risk' of bias.
Insufficient information about the sequence generation process to permit judgement of 'low risk' or 'high risk'.

\section{Allocation concealment}

Selection bias (biased allocation to interventions) due to inadequate concealment of allocations prior to assignment

Criteria for a judgement of 'low risk' of bias
Participants and investigators enrolling participants could not foresee assignment because one of the following, or an equivalent method, was used to conceal allocation:

1. central allocation (including telephone, web-based and pharmacy-controlled randomisation);

2. sequentially numbered drug containers of identical appearance;

3. sequentially numbered, opaque, sealed envelopes.
Criteria for the judgement of 'high risk' of bias
Participants or investigators enrolling participants could possibly foresee assignments and thus introduce selection bias, such as allocation based on:

1. using an open random allocation schedule (e.g. a list of random numbers);

2. assignment envelopes were used without appropriate safeguards (e.g. if envelopes were unsealed or nonopaque or not sequentially numbered);

3. alternation or rotation;

4. date of birth;

5. case record number;

6. any other explicitly unconcealed procedure.
Criteria for the judgement of 'unclear risk' of bias
Insufficient information to permit judgement of 'low risk' or 'high risk'. This is usually the case if the method of concealment is not described or not described in sufficient detail to allow a definite judgement - for example if the use of assignment envelopes is described, but it remains unclear whether envelopes were sequentially numbered, opaque and sealed.

\section{Blinding of participants and personnel}

Performance bias due to knowledge of the allocated interventions by participants and personnel during the study

\section{Criteria for a judgement of} 'low risk' of bias
Any one of the following:

1. no blinding or incomplete blinding, but the review authors judge that the outcome is not likely to be influenced by lack of blinding;

2. blinding of participants and key study personnel ensured, and unlikely that the blinding could have been broken. 
(Continued)

Criteria for the judgement of 'high risk' of bias
Any one of the following:

1. no blinding or incomplete blinding, and the outcome is likely to be influenced by lack of blinding;

2. blinding of key study participants and personnel attempted, but likely that the blinding could have been broken, and the outcome is likely to be influenced by lack of blinding.
Criteria for the judgement of 'unclear risk' of bias
Any one of the following:

1. insufficient information to permit judgement of 'low risk' or 'high risk';

2. the study did not address this outcome.

\section{Blinding of outcome assessment}

Detection bias due to knowledge of the allocated interventions by outcome assessors

$\begin{array}{ll}\begin{array}{l}\text { Criteria for a judgement of } \\ \text { 'low risk' of bias }\end{array} & \begin{array}{l}\text { Any one of the following: } \\ \text { is no blinding of outcome assessment, but the review authors judge that the outcome measurement } \\ \text { 2. blinding of outcome assessment ensured, and unlikely that the blinding could have been broken. }\end{array}\end{array}$

Criteria for the judgement of 'high risk' of bias
Any one of the following:

1. no blinding of outcome assessment, and the outcome measurement is likely to be influenced by lack of blinding;

2. blinding of outcome assessment, but likely that the blinding could have been broken, and the outcome measurement is likely to be influenced by lack of blinding.
Criteria for the judgement of
Any one of the following:
'unclear risk' of bias
1. insufficient information to permit judgement of 'low risk' or 'high risk';
2. the study did not address this outcome.

\section{Incomplete outcome data}

Attrition bias due to amount, nature or handling of incomplete outcome data

\section{Criteria for a judgement of} 'low risk' of bias

\section{Any one of the following:}

1. no missing outcome data;

2. reasons for missing outcome data unlikely to be related to true outcome (for survival data, censoring unlikely to be introducing bias);

3. missing outcome data balanced in numbers across intervention groups, with similar reasons for missing data across groups;

4. for dichotomous outcome data, the proportion of missing outcomes compared with observed event risk not enough to have a clinically relevant impact on the intervention effect estimate;

5. for continuous outcome data, plausible effect size (difference in means or standardised difference in means) among missing outcomes not enough to have a clinically relevant impact on observed effect size;

6. missing data have been imputed using appropriate methods.
Any one of the following:

1. reason for missing outcome data likely to be related to true outcome, with either imbalance in numbers or reasons for missing data across intervention groups;

2. for dichotomous outcome data, the proportion of missing outcomes compared with observed event risk enough to induce clinically relevant bias in intervention effect estimate; 
3. for continuous outcome data, plausible effect size (difference in means or standardised difference in means) among missing outcomes enough to induce clinically relevant bias in observed effect size;

4. 'as-treated' analysis done with substantial departure of the intervention received from that assigned at randomisation;

5. potentially inappropriate application of simple imputation.

Criteria for the judgement of 'unclear risk' of bias

\section{Any one of the following:}

1. insufficient reporting of attrition/exclusions to permit judgement of 'low risk' or 'high risk' (e.g. number randomised not stated, no reasons for missing data provided);

2. the study did not address this outcome.

\section{Selective reporting}

Reporting bias due to selective outcome reporting

Criteria for a judgement of 'low risk' of bias
Any of the following:

1. the study protocol is available and all of the study's prespecified (primary and secondary) outcomes that are of interest in the review have been reported in the prespecified way;

2. the study protocol is not available but it is clear that the published reports include all expected outcomes, including those that were prespecified (convincing text of this nature may be uncommon).
Criteria for the judgement of 'high risk' of bias

\section{Any one of the following:}

1. not all of the study's prespecified primary outcomes have been reported;

2. one or more primary outcomes is reported using measurements, analysis methods or subsets of the data (e.g. subscales) that were not prespecified;

3. one or more reported primary outcomes were not prespecified (unless clear justification for their reporting is provided, such as an unexpected adverse effect);

4. one or more outcomes of interest in the review are reported incompletely so that they cannot be entered in a meta-analysis;

5. the study report fails to include results for a key outcome that would be expected to have been reported for such a study.
Criteria for the judgement of 'unclear risk' of bias
Insufficient information to permit judgement of 'low risk' or 'high risk'. It is likely that the majority of studies will fall into this category.

\section{Other bias}

Bias due to problems not covered elsewhere in the table
Criteria for a judgement of
The study appears to be free of other sources of bias.

'low risk' of bias

Criteria for the judgement of

'high risk' of bias
There is at least one important risk of bias. For example, the study:

1. had a potential source of bias related to the specific study design used; or

2. has been claimed to have been fraudulent; or

3. had some other problem.
Criteria for the judgement of 'unclear risk' of bias
There may be a risk of bias, but there is either:

1. insufficient information to assess whether an important risk of bias exists; or

2. insufficient rationale or evidence that an identified problem will introduce bias. 


\section{HISTORY}

Protocol first published: Issue 11, 2017

Review first published: Issue 5, 2020

\section{CONTRIBUTIONS OFAUTHORS}

1. Drafting the protocol and review: MMG, MAT, CC, MMC

2. Selecting studies: MMG and MAT

3. Extracting data from studies: MMG and MAT

4. Entering data into RevMan: MMG

5. Carrying out the analysis: MMG, MAT and CC

6. Interpreting the analysis: MMG, MAT and CC

7. Drafting the final review: MMG, MAT, CC and MMC

8. Resolving disagreements: MMG, MAT, CC and MMC

\section{DECLARATIONS OF INTEREST}

MMG: none known

CC: none known

MMC: none known

MAT: none known

\section{SOURCES OF SUPPORT}

\section{Internal sources}

- Centre for Public Health, Queens University Belfast, UK

\section{External sources}

- Public Health Agency, HSC R \& D Division, UK

Provision of funding for the project

\section{DIFFERENCES BETWEEN PROTOCOL AND REVIEW}

We had planned to report adverse events as a dichotomous outcome. As adverse events were inconsistently measured and reported, this precluded meta-analysis. We therefore provided a narrative description of adverse events. We did not include overall survival and recurrence-free survival in our 'Summary of findings' table (as indicated in the protocol) because it was not reported in any of the included studies. In measures of treatment effect, we modified the description of our follow-up periods to further clarify our follow-up time points, e.g. up to 12 weeks follow-up became up to or equal to 12 weeks follow-up. We used the term 'physical activity interventions' throughout the review to encompass exercise interventions for readers ease. We made reference to this in the description of interventions section. We made minor changes to the data extraction form that was proposed in the protocol after testing the pre developed form on a random sample of studies. Subgroup analysis were precluded, and we therefore removed any reference to subgroup analysis from the Assessment of heterogeneity section. We made a minor amendment to the wording under the Types of participants section, as the wording inferred patients that were not treated surgically would not be included in the review; this was not the case and was an oversight by the authors during protocol development. We included three studies that had a health education component in addition to a physical activity intervention, inclusion of these studies was unforeseen and therefore not identified in the protocol. Authors met to discuss inclusion of these studies and agreed to include studies and investigate the effect of inclusion in sensitivity analysis, where appropriate. The effect of including these studies is discussed in Types of interventions, Quality of the evidence and Potential biases in the review process sections. 